\title{
Structure Based Search for Novel Nuclear Export Inhibiting Anti-Tumor Drugs
}

\section{Dissertation}

\author{
for the award of the degree \\ "Doctor rerum naturalium" \\ of the Georg-August-Universität Göttingen \\ within the doctoral program \\ "Biomolecules: Structure - Function - Dynamics" \\ of the Georg-August-University School of Science (GAUSS)
}

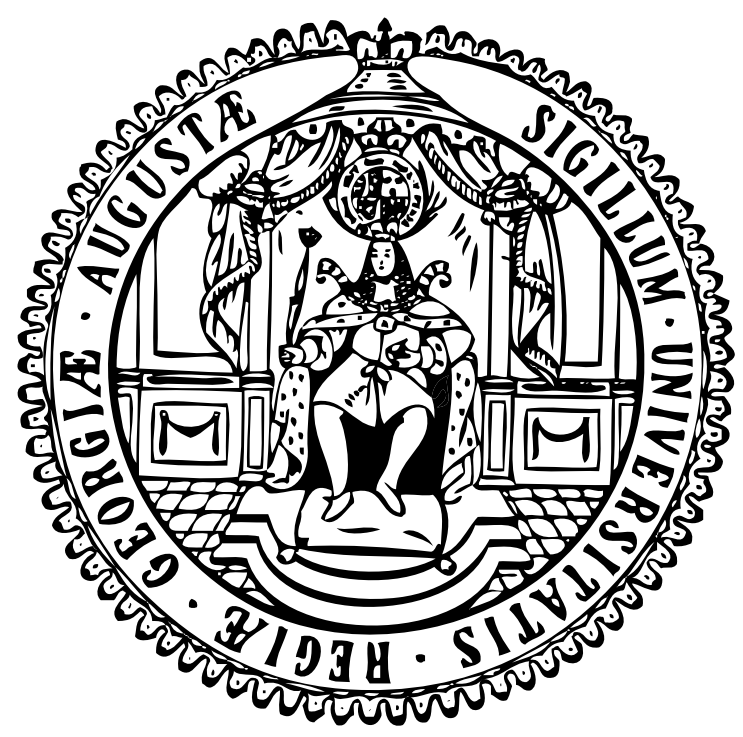

submitted by

Alaa Shaikhqasem

from Ramallah, Palestinian Territories

Göttingen, 2020 
Members of the Thesis Advisory Committee

\section{Prof. Dr. Ralf Ficner (Reviewer 1)}

Georg-August-Universität Göttingen

Institute for Microbiology and Genetics

Department for Molecular Structural Biology

\section{Prof. Dr. Ralph H. Kehlenbach (Reviewer 2)}

Georg-August-Universität Göttingen

Department of Molecular Biology

\section{Prof. Dr. Kai Tittmann}

Georg-August-Universität Göttingen

Schwann-Schleiden Research Centre

Department of Molecular Enzymology

Members of the Examination Board

\section{Prof. Dr. Heike Krebber}

Georg-August-Universität Göttingen

Institute for Microbiology and Genetics

Department of Molecular Genetics

Prof. Dr. Gerhard Braus

Georg-August-Universität Göttingen

Institute for Microbiology and Genetics

Department of Molecular Microbiology and Genetics

\section{Prof. Dr. Peter Rehling}

University Medical Center Göttingen

Cellular Biochemistry 


\section{Statutory declaration:}

I hereby declare that I prepared the doctoral thesis titled "Structure Based Search for Novel Nuclear Export Inhibiting Anti-Tumor Drugs" independently and with no sources and aids other than quoted. The thesis has not been submitted elsewhere.

Alaa Shaikhqasem, April 2020 


\section{Contents}

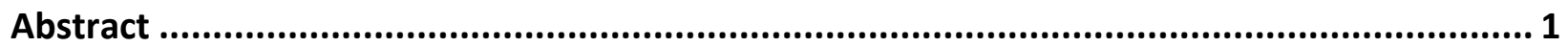

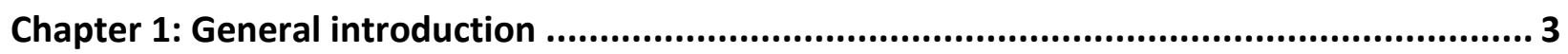

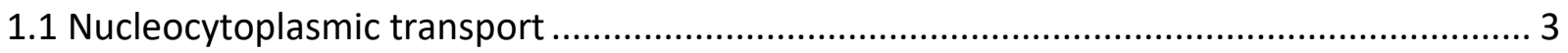

1.1.1 The nuclear pore complex .................................................................................... 3

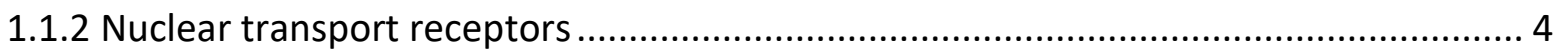

1.1.3 The Ran cycle ......................................................................................................... 5

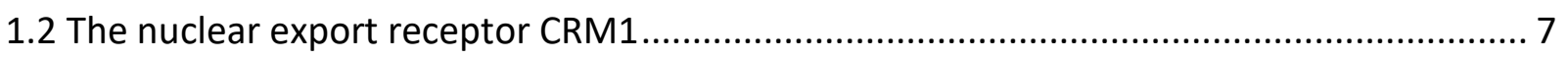

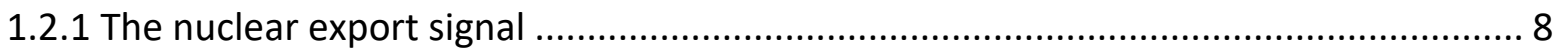

1.2.2 The overall structural arrangement of CRM1 - cargo interactions ............................... 9

1.2.3 Structural and conformational flexibility of CRM1 ...................................................... 11

1.2.4 CRM1 interaction with the nuclear pore complex ................................................. 13

1.3 Altered nucleocytoplasmic distribution of proteins in cancer ............................................ 15

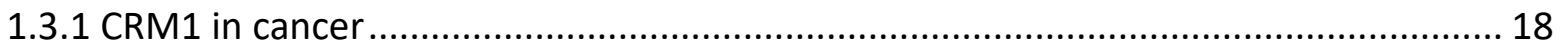

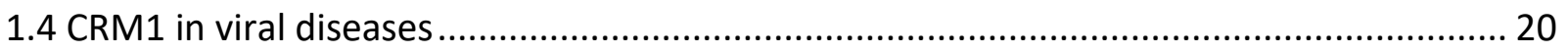

1.5 Inhibition of CRM1-mediated nuclear export ............................................................... 21

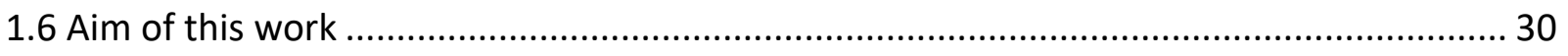

Chapter 2: Characterization of inhibition reveals distinctive properties for human and Saccharomyces cerevisiae CRM1 ............................................................................................ 32

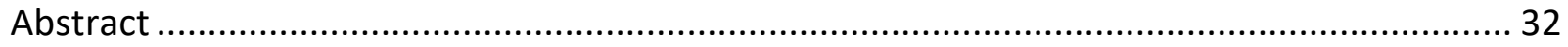

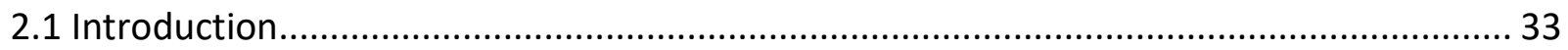

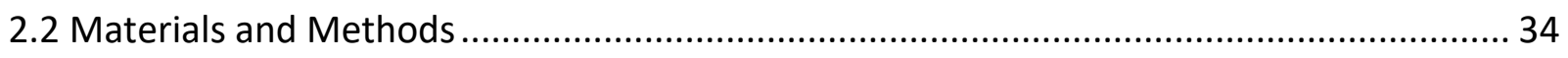

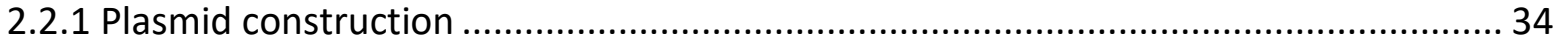

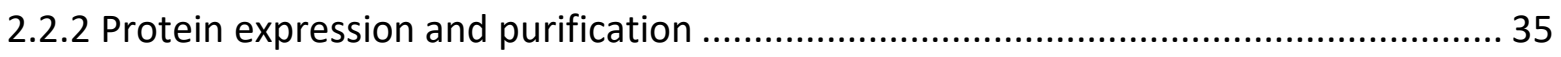

2.2.3 Preparation and crystallization of ${ }^{H s}$ CRM1-Hs $R$ Ran GTP-LMB complex .......................... 36

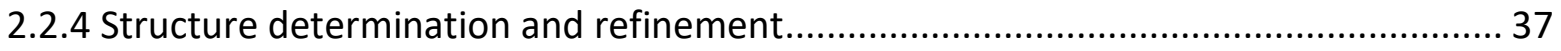

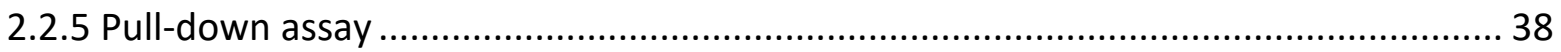

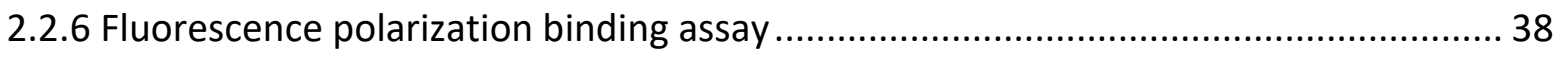

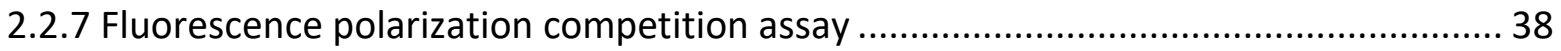

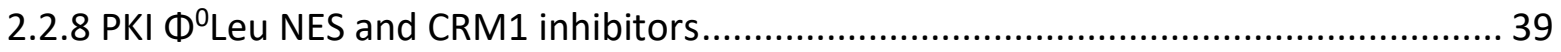




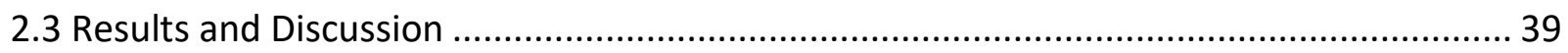

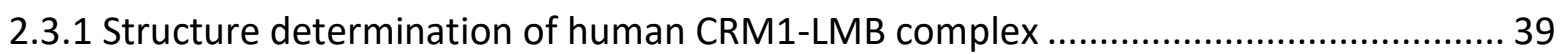

2.3.2 Comparison of LMB binding in human CRM1 and Saccharomyces cerevisiae CRM1 .. 42

2.3.3 Human and Saccharomyces cerevisiae CRM1-inhibitor interaction patterns reveal high structural and sequence similarities.................................................................... 43

2.3.4 Compounds $\mathrm{C} 3, \mathrm{C} 6$ and $\mathrm{C} 10$ inhibit CRM1 by direct binding in the NES-binding cleft in

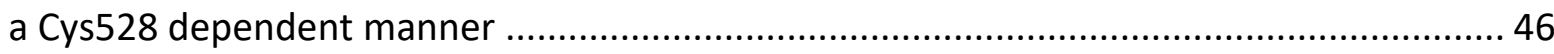

2.3.5 DP2392-E10 exhibits a unique binding mode that is not dependent on Cys528........ 48

2.3.6 Human and yeast CRM1 reveal different binding affinities towards PKI $\Phi^{0}$ Leu NES .. 49

2.3.7 Thr539Cys ${ }^{S c} \mathrm{CRM} 1$ mutant binds strongly LMB but not compounds C3 and C6. ....... 51

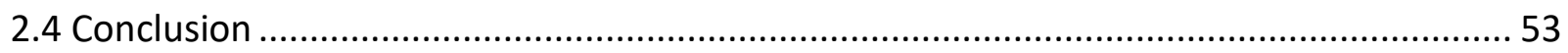

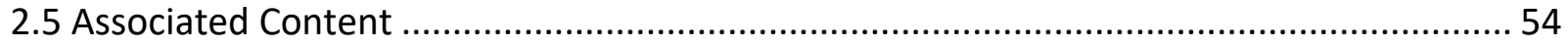

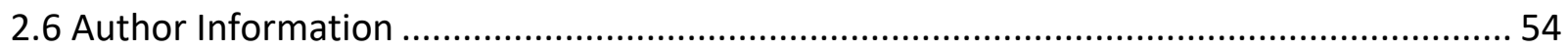

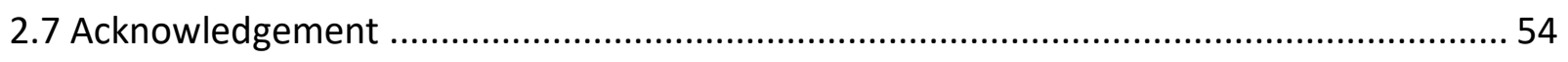

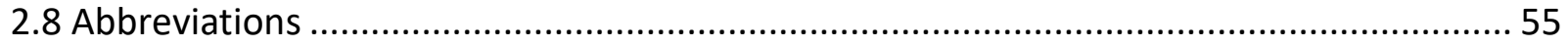

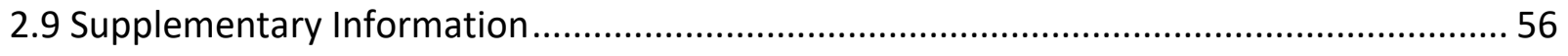

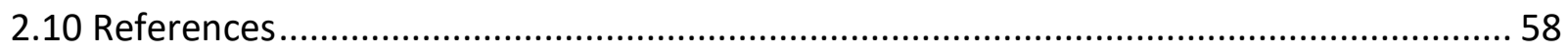

Chapter 3: Molecular docking of novel nuclear export inhibitors reveals an allosteric mechanism of human CRM1 inhibition .............................................................. 63

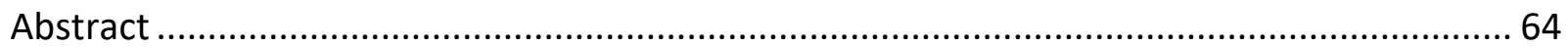

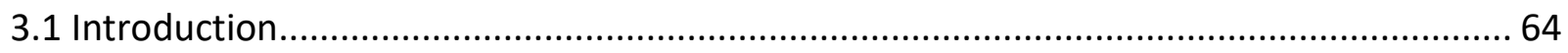

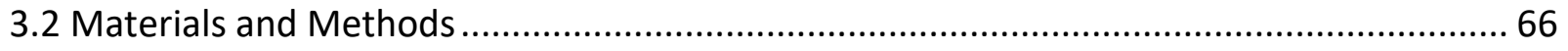

3.2.1 Protein preparation and CRM1-inihibitor complexes crystallization ........................ 66

3.2.2 ${ }^{H s}{ }^{H}$ CRM1 ${ }^{\Delta-R a n G T P-C 6 ~ c o m p l e x ~ c r y s t a l s ~ d e h y d r a t i o n ~ a n d ~ p r e p a r a t i o n ~ . . . . . . . . . . . . . . . . . . . . . . . ~} 67$

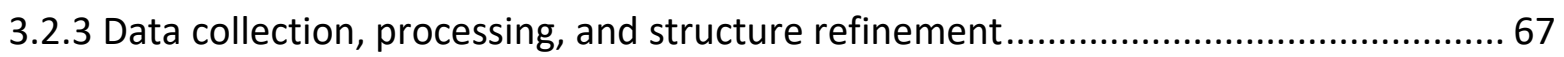

3.2.4 Identification of cysteine modification by liquid chromatography-mass spectrometry

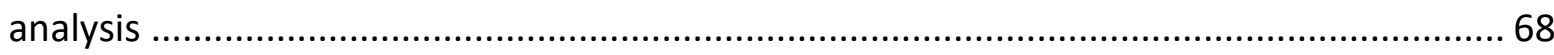

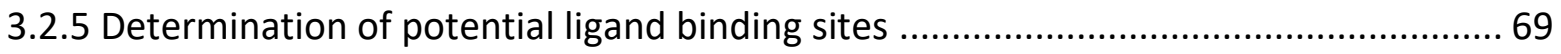

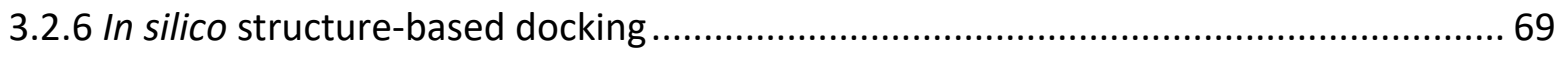

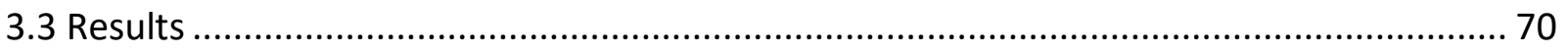

3.3.1 Crystallization of human CRM1-inhibitor complexes ........................................... 70

3.3.2 Modification of Cys528 by 2-Mercaptoethanol .................................................. 75 
3.3.3 Predicted binding conformations of the compounds $\mathrm{C} 3, \mathrm{C} 6$, and $\mathrm{C} 10$ in the NESbinding cleft

3.3.4 Human CRM1 exhibits multiple potential ligand binding sites outside the NES-binding cleft 80

3.3.5 DP2392-E10 docking demonstrates unique binding interactions at the base of the Acidic loop 81

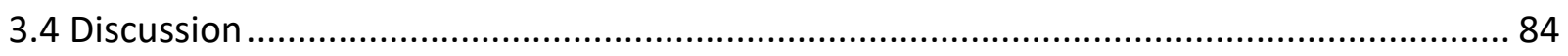

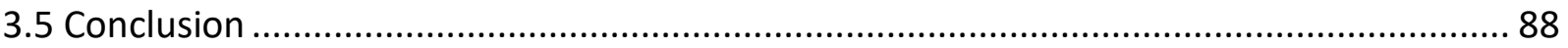

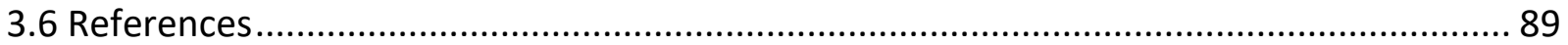

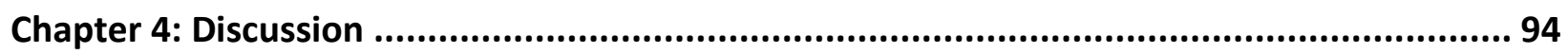

4.1 High conformational stability is a major determinant of human CRM1 crystallizability.... 95 4.2 Molecular docking predicts a similar binding mode of C3 and C6 to SINEs KPT compounds

4.3 Alternative mechanisms of CRM1 mediated nuclear export inhibition ......................... 98

4.3.1 Allosteric modulation of the NES-binding cleft ...................................................... 98

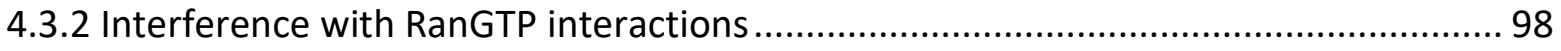

4.3.3 Interfering CRM1 translocation through the nuclear pore complex ....................... 99

4.4 Structure-based methods in the discovery and development of CRM1 targeting drugs. 101

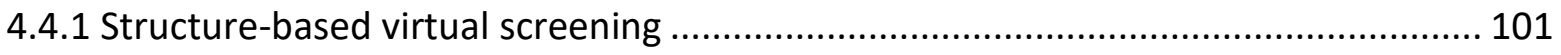

4.4.2 Crystallographic fragment screening.......................................................... 102

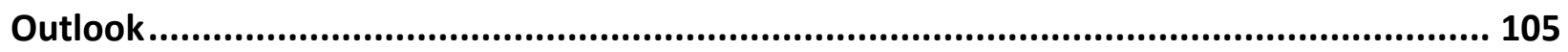

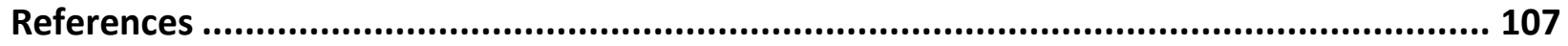

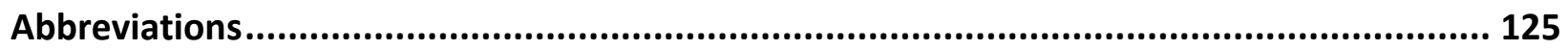

Acknowledgements ............................................................................................. 129 


\section{Abstract}

The intracellular distribution of proteins and ribonucleoproteins is a fundamental process in all eukaryotes that is critically vital for the normal functionality of the eukaryotic cell. In the last decades, it has been discovered that cancer cells utilize the transport machinery to stimulate tumor growth and to effectively evade apoptotic mechanisms. Chromosome region maintenance 1 (CRM1) is a major nuclear export receptor that was found to mediate the mislocalization of several tumor suppressor proteins such as Rb, APC, p53, p21 and p27 as well as cancer drug targets like topoisomerase II $\alpha$. In addition, elevated CRM1 expression has been observed in several cancers and was correlated with poor patient prognosis. This renders CRM1 a particularly interesting target for therapeutic intervention in diverse cancer diseases. In the recent years, structural characterization of CRM1 inhibitor complexes has been performed exclusively using CRM1 from the yeast Saccharomyces cerevisiae. The yeast protein was genetically modified to mimic its human homolog by the incorporation of a cysteine residue that is required for inhibitor binding. This approach has been used as a model system for the structural characterization of several natural and synthetic inhibitors bound to yeast CRM1. The performed studies defined a typical mechanism of CRM1 inhibition by the covalent modification of a reactive cysteine residue that is located in the nuclear export signal (NES)-binding cleft (Cys528 in human). The aim of this work was to gain structural and biochemical insight into the Inhibition of human CRM1, the actual protein targeted for anti-tumor drug development. This study focused on the biochemical and structural characterization of four novel CRM1 inhibitor candidates: the compounds C3, C6, C10 and DP2392-E10. In addition, during this work Leptomycin B (LMB), a well-studied CRM1 inhibitor, was implemented for the development of a successful crystallization approach of human CRM1 - inhibitor complexes. Genetic modifications coupled with intensive screening for potential crystallization conditions succeeded to gain the crystal structure of LMB bound to human CRM1-RanGTP complex. The obtained structure revealed that LMB interactions with human CRM1 are rather similar but not identical to those with the yeast variant. Moreover, binding assays showed that some of the tested novel compounds as well as a NES peptide exhibit no binding or lower binding affinity towards yeast CRM1 when compared to the human protein. Introducing the reactive cysteine to yeast CRM1 did not lead to the binding of some of the tested compounds, indicating that the used model system is limited to a certain class of human CRM1 inhibitors. Furthermore, during this work several experimental and computational methods were applied using human CRM1 to elucidate the inhibitory mechanisms of C3, C6, C10 and DP2392E10. Quantitative binding assays revealed that all four compounds bind directly to CRM1 in a 
concentration dependent manner. Further analysis unveiled that CRM1 inhibition by C3, C6 and C10 is mediated by the reactive cysteine (Cys528), which indicated they act by the direct blocking of the NES-binding cleft. In contrast, DP2392-E10 exhibited unique binding properties that are not dependent on Cys528. Computational docking, using 'ligand free' human CRM1-LMB complex structure as a template, defined the binding of DP2392-E10 outside the NES-binding cleft, at the base of the acidic loop. The identified binding mode suggests a novel mechanism of CRM1 inhibition by the allosteric modulation of the NES-binding cleft. Moreover, computational analysis on human CRM1 identified several potential ligand binding sites outside the NES-binding cleft, which indicates for potential alternative mechanisms for the inhibition of CRM1-mediated nuclear export.

The work presented here provides new insights into human CRM1 inhibition and it emphasizes the importance of using the human protein for inhibitor studies. Furthermore, the crystal structure of CRM1-RanGTP-LMB complex obtained during this study represents a valuable framework for different experimental and computational methods that can be applied for CRM1targeting drug design. 


\section{Chapter 1: General introduction}

A hallmark of eukaryotes is the development of an endomembrane system which resulted in the compartmentalization of the cell and the formation of several organelles such as the mitochondria, Golgi apparatus, the endoplasmic reticulum, and a main central compartment, the nucleus. In the eukaryotic cell the nucleus encloses the genetic material by a double membrane (nuclear envelope) resulting in the spatial separation of several molecular processes mainly transcription and translation. The physical separation allows the tight control and the high regulation of multiple cellular process and provide the suitable biochemical environment required for their optimal activity. However, the spatial separation of these biological events created the need to exchange a variety of molecules between both compartments, like for example the nuclear export of RNAs and ribosomal subunits as well as the nuclear import of polymerases, histones, and transcription factors. Transport events between the nucleus and the cytoplasm take place through nuclear portals known as nuclear pore complexes (NPCS) in a process referred to as the nucleocytoplasmic transport. The trafficking of molecules between both compartments is a fundamental process in all eukaryotes and crucial for maintaining the proper intracellular distribution of proteins and ribonucleoprotein (RNP) complexes. This in turn is essential for the proper functionality of the eukaryotic cells and therefore affect every aspect of human health and disease. (Chook et al. 1999; Conti and Izaurralde 2001; Dickmanns, Monecke, and Ficner 2015; Peters 2006; Sun et al. 2016)

\subsection{Nucleocytoplasmic transport}

\subsubsection{The nuclear pore complex}

The NPCs are aqueous channels that are embedded within the nuclear envelop. They are among the largest macromolecular complexes in the cell (over $120 \mathrm{MDa}$ in size in human) and formed by the assembly of multiple copies of around 30 different proteins named nucleoporins (NUPs) (Cronshaw et al. 2002; Hoelz, Debler, and Blobel 2011). The general structure of the NPC demonstrates a central channel with a characteristic eight-fold rotational symmetry (Yang, Rout, and Akey 1998). The central channel of the NPC is composed by three inner rings stacked into the nuclear envelope and bordered by an outer cytoplasmic ring and an outer nuclear ring (Figure 1). The cytoplasmic ring is characterized by a series of flexible filaments protruding to the cytoplasmic side of the NPC (cytoplasmic filaments), while the nuclear ring serves as an attachment site of a basket like structure comprised of eight filaments joined with a distal ring 
(Figure 1) (nuclear basket) (Lin et al. 2018). The nuclear and the cytoplasmic rings are structurally connected to the inner ring by bridging Nup155 molecules. Whereas, the assembly of the symmetric core is mediated via the interactions of the flexible linker sequences in Nup98, Nup53, and Nup93 (Fischer et al. 2015; Lin et al. 2016; Stuwe et al. 2015). NUPs forming the inner central channel of the pore contain intrinsically disordered domains rich in phenylalanine-glycine (FG repeats) and therefore called FG-nucleoporins (FG-NUPs). FG repeats within the central channel were shown to form an efficient selective barrier that allows only proteins of a certain size to freely diffuse across the NPC in a reasonable amount of time (Görlich and Mattaj 1996; Schmidt and Görlich 2016).

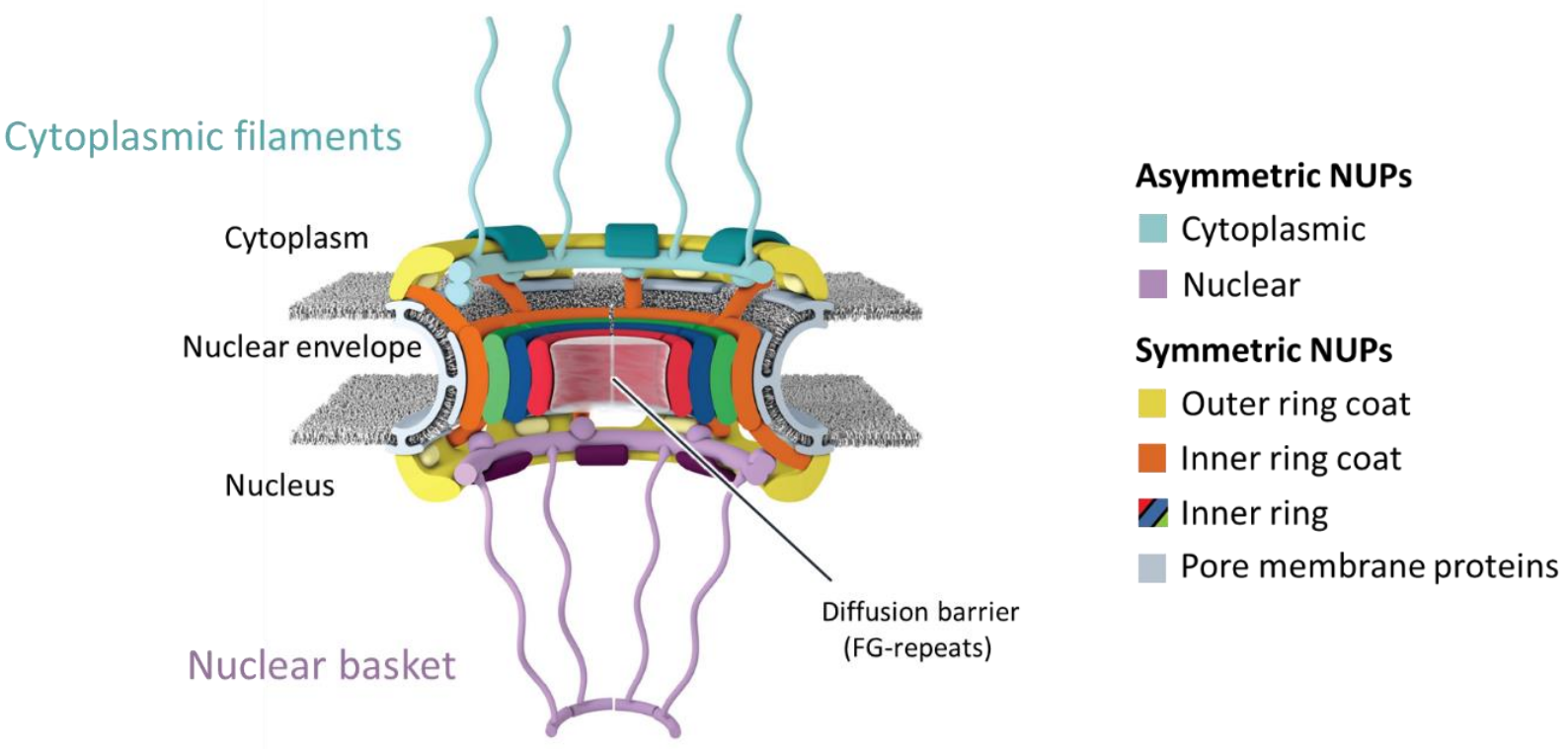

Figure 1: Simplified schematic representation of different structural elements of the nuclear pore complex. The inner ring coat, composed mainly of Nup155, connects the outer nuclear and cytoplasmic rings with the inner ring. The pore is anchored to the nuclear envelope by a layer of pore membrane proteins that surrounds the inner ring. The diffusion barrier within the central channel is formed by the FG-repeats meshwork. The figure is modified from (Lin and Hoelz 2019).

\subsubsection{Nuclear transport receptors}

The selectivity barrier created by the complex arrangement of FG-repeats allows only for most molecules that are smaller than $40 \mathrm{kDa}$ in size or less than $5 \mathrm{~nm}$ in diameter to freely transverse the NPC (Bonner 1975; Mohr et al. 2009; Schmidt and Görlich 2016). The transport of larger molecules which include a multitudinous amount of proteins and ribonucleoprotein complexes is mediated by transport receptors (karyopherins) (Cook et al. 2007; Görlich and Kutay 1999). Transport receptors are members of the importin $\beta$ superfamily and are relatively large proteins 
that exhibit a molecular weight of about 90-150 kDa. In human there are more than 20 transport receptors of importin $\beta$ superfamily identified. While some of these receptors can mediate bidirectional transport from and into the nucleus, most of them demonstrate a unidirectional transport and are classified into import receptors (importins) or export receptors (exportins) according to the direction of transport with respect to the nucleus (Harel and Forbes 2004). Importins and exportins share several common structural and functional properties. Besides their cargo proteins, they bind a small GTPase Ras-related antigen (Ran), which plays an essential role in regulating the directionality of transport (Görlich et al. 1996; Rexach and Blobel 1995). Furthermore, at a structural level karyopherins consist of a tandem of repetitive elements named HEAT repeats. A HEAT repeat is a structural motif that consists of 40 - 50 residues that are arranged as two antiparallel helices (named A-helix and B-helix) connected by a short linker loop. The name HEAT was given after the 4 proteins where this motif was first identified: Huntingtin, elongation factor 3 (EF3), protein phosphatase 2A (PP2A), and the P3 kinase TOR1 (Andrade and Bork 1995; Andrade, Perez-Iratxeta, and Ponting 2001).

Transport receptors recognizes their cargo proteins via a sequence specific signal peptide known as Nuclear Localization Signal (NLS) for importins and Nuclear Export Signal (NES) for exportins (Kutay, Guttinger, and Gorlich 2005; Lange et al. 2007). The cargo proteins can directly bind the transport receptor or alternatively the interaction is mediated via adapter molecules. The most studied import receptor is the Importin $\alpha /$ Importin $\beta$ heterodimer, which bind cargo proteins bearing a classical NLS (cNLS) peptide that consists of a series of basic residues (Soniat and Chook 2015; Tran, King, and Corbett 2014). The most well understood and thus most described export receptor is exportin 1, known also as Chromosome region maintenance 1 (CRM1). CRM1 mediates the nuclear export of cargos bearing a leucine-rich NES that contains a set of hydrophobic residues with characteristic spacing (Will be described in more detail in following sections) (Güttler et al. 2010; Monecke, Dickmanns, and Ficner 2014).

\subsubsection{The Ran cycle}

The directionality of transport between the nucleus and the cytoplasm is highly regulated by the small GTPase Ras-related antigen Ran, depending on its bound nucleotide either GDP (RanGDP) or GTP (RanGTP) (Görlich et al. 1996; Rexach and Blobel 1995). The nuclear export of a certain cargo is dependent on the cooperative binding of RanGTP and the cargo protein to the exportin for the formation of a stable ternary export complex. Once the export complex is formed, it transverses the NPC to the cytoplasm where the conversion of RanGTP to RanGDP lead to 
complex dissociation and the termination of the export process (Figure 2) (Fornerod, Ohno, et al. 1997; Kehlenbach et al. 1999; Monecke et al. 2014). On the other hand, importins recruit their cargos in the cytoplasm and cross through the NPC as binary complexes. In contrast to exportins, the binding of RanGTP to importins in the nucleus induces cargo release and import termination (Görlich et al. 1996; Rexach and Blobel 1995).

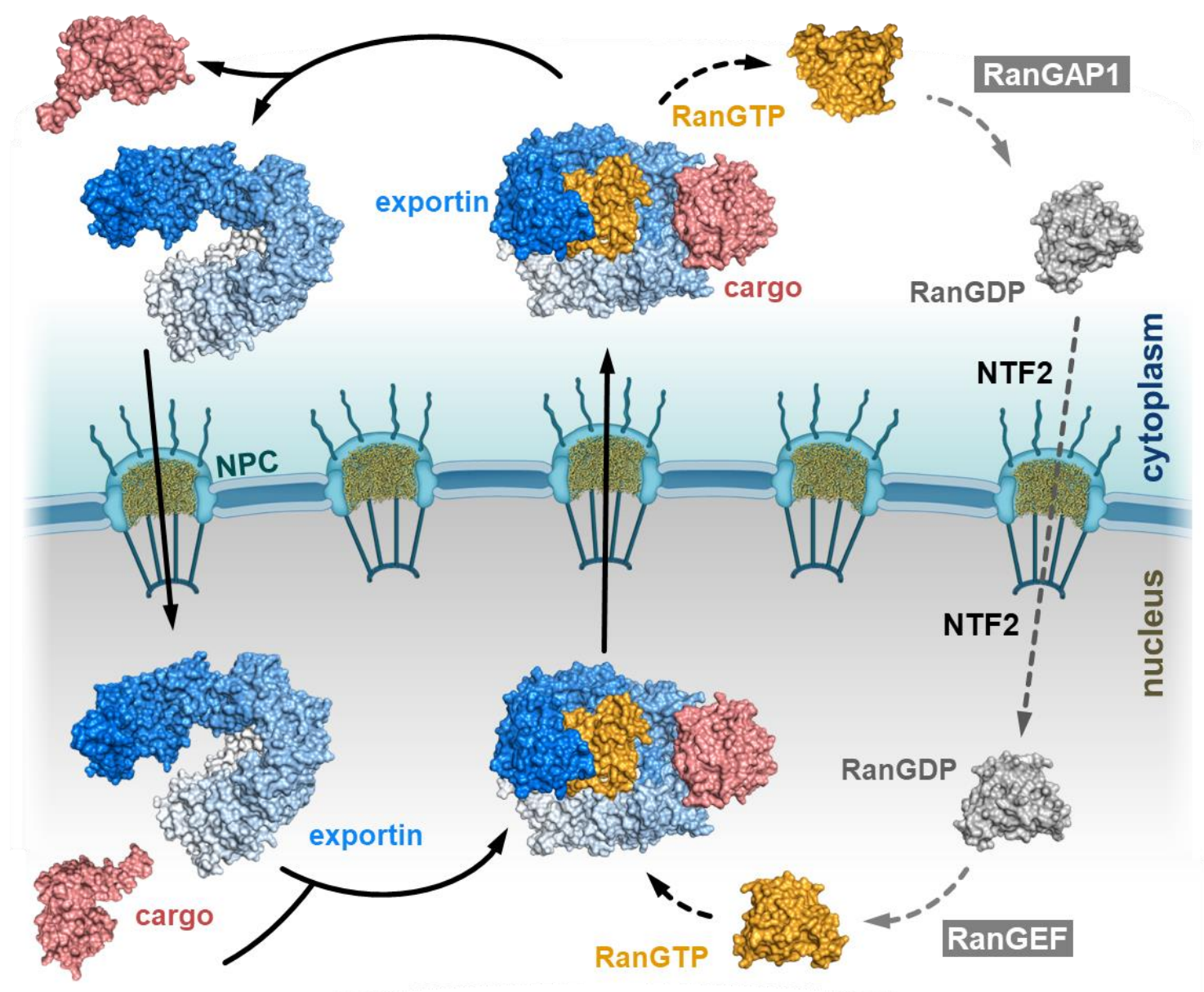

Figure 2: Schematic representation of nuclear export cycle by Importin $\beta$ type nuclear export receptors across the nuclear pore complexes (NPCs). In the nucleus the cargo protein and RanGTP bind cooperatively to the exportin (gradient colored marine blue - white) and form a stable ternary export complex. The ternary complex crosses the NPC to the cytoplasm, where the conversion of RanGTP to RanGDP mediated by RanGAP1 leads to complex dissociation and export termination. RanGDP is actively transported back to the nucleus by NTF2. In the nucleus the constant high concentration of RanGTP is maintained by the activity of RanGEF. The figure is modified from (Monecke et al. 2014).

A gradient of RanGDP/RanGTP is maintained across the nuclear envelope with a 200-1000 folds higher RanGTP concentration in the nucleus. This gradient is achieved and sustained by the asymmetric distribution of Ran regulatory factors, Ran GTPase activating protein (RanGAP1) in 
the cytoplasm and Ran guanine nucleotide exchange factor (RanGEF) in the nucleus (Güttler and Görlich 2011; Kalab, Weis, and Heald 2002; Smith et al. 2002). In the cytoplasm RanGAP1 accelerate Ran GTPase activity by a factor of 10000. In the presence of the Ran binding protein 1 (RanBP1) the GTPase activity was observed to be further increased (Bischoff et al. 1995). RanGDP is actively imported to the nucleus by nuclear transport factor 2 (NTF2) (Smith, Brownawell, and Macara 1998). In the nucleus the conversion of RanGDP to RanGTP is mediated by RanGEF (Figure 2) which is also known as RCC1 (regulator of chromosome condensation 1) as it was found to associate to chromatin (Bischoff and Ponstingl 1991; Makde et al. 2010) . Thereby the gradient of RanGTP/RanGDP across the nuclear envelope determine the directionality of transport by regulating receptor-cargo interactions.

\subsection{The nuclear export receptor CRM1}

CRM1 is an export receptor with a molecular weight of 120 kDa (1071 residues in human, 123.4 $\mathrm{kDa}$ ) and it mediates the nuclear export of a wide range of structurally and functionally unrelated proteins and RNP complexes. CRM1 was originally identified as a mutated gene in a cold sensitive strain of the yeast Schizosaccharomyces pombe, where mutations in the gene led to deformed nuclear chromosome domains (Adachi and Yanagida 1989). Further investigations revealed that CRM1 mutations lead to the deregulation of a transcription factor called pap1 (Toda et al. 1992). The first clue on CRM1 activity in the process of nucleocytoplasmic transport was indicated by its binding to the nucleoporin Nup214 (also known as CAN), which is localized at the cytoplasmic side of the NPC (Fornerod, Van Deursen, et al. 1997). Later experiments performed in vivo and in vitro confirmed CRM1 function as a nuclear export receptor and identified several CRM1 cargos by their NES sequences (Fukuda et al. 1997; Kehlenbach, Dickmanns, and Gerace 1998; OssarehNazari, Bachelerie, and Dargemont 1997; Stade et al. 1997). Moreover, CRM1 was found to be an essential protein in all tested organisms. Besides its role as a nuclear exporter receptor, several studies showed CRM1 is involved in other non-transport cellular functions. For example, CRM1 was shown to be implicated in various steps during mitosis; it is localized at the kinetochores from the end of prophase to late anaphase, where it binds RanGAP and the nucleoporin Nup358 (also called Ran binding protein 2 (RanBP2)) (Arnaoutov et al. 2005; Di Fiore, Ciciarello, and Lavia 2004). In addition, CRM1 is also localized at the centrosome and it was shown to play an important role in the tight regulation of centrosomal duplication (Wang et al. 2005). Nevertheless, apart from its cellular functions, CRM1 was found to be co-opted by viruses from several viral families during different stages of the of viral replication cycle. Moreover, in several cancers CRM1 was identified for the cytoplasmic mislocalization of various oncoproteins and tumor suppressor proteins. This rendered CRM1 an appreciated target for the development of 
anti-tumor and anti-viral drugs (Dickmanns et al. 2015; Hill et al. 2014; Mathew and Ghildyal 2017).

Proteomic studies on CRM1-mediated export identified over a 1000 potential CRM1 cargoes in human and around 700 potential cargoes in the yeast Saccharomyces cerevisiae (Kırlı et al. 2015). The complexity of CRM1 cargo ranges from a single protein to a multimeric RNP complexes. The export of a simple cargo protein can be achieved by the direct binding of the cargo to CRM1 like the export of the import adapter Snurportin 1 (SPN1) (Monecke et al. 2009; Paraskeva et al. 2000). On the other hand, more complicated export events require additional factors and adaptor proteins. For example, the nuclear export of $\mathrm{m}^{7} \mathrm{G}$-capped small nuclear RNAs (snRNAs) requires additionally PHAX (phosphorylated adapter of RNA export) as well as the cap binding proteins (CBP20 and CPB80) (Ohno et al. 2000; Segref, Mattaj, and Ohno 2001). Another example is the export of pre-60S ribosomal subunits (rSUs), which requires the export adapter Nmd3 (Ho, Kallstrom, and Johnson 2000).

\subsubsection{The nuclear export signal}

CRM1 recognizes the cargoes and the adapter proteins by their leucine - rich NES peptide. The NES peptide comprises 4-5 hydrophobic residues (Ф0 - Ф4) with characteristic spacing following the consensus $\Phi 1-(X)_{2-3}-\Phi 2-(X)_{2-3}-\Phi 3-X-\Phi 4$, where $X$ is any amino acid and $\Phi$-residues are basically leucines but can also be isoleucine, valine, phenylalanine or methionine (Kutay et al. 2005). NESes were first identified in the human immunodeficiency virus type 1 (HIV-1)-Rev protein and in protein kinase A inhibitor (PKI) (Fischer et al. 1995; Wen et al. 1995). Afterwards, NESes were identified in several cargoes and were classified based on the spacing pattern of the critical hydrophobic residues into the classes: 1a, 1b, 1c, 1d, 2, 3, and 4 (Figure 3) (Kosugi et al. 2008; Lee et al. 2019).

\begin{tabular}{|c|c|c|c|}
\hline Class 1a & Ф1 XXX & ( $12 \mathrm{XX}$ & Ф3 $\mathrm{X}$ \\
\hline Class 1b & Ф1 XX & $\Phi 2 \mathrm{XX}$ & Ф3 $\mathrm{X}$ \\
\hline Class 1c & Ф1 XXX & $\Phi 2 \mathrm{XXX}$ & Ф3 $\mathrm{X}$ \\
\hline Class 1d & Ф1 XX & $\Phi 2 \mathrm{XXX}$ & (\$3 X \\
\hline Class 2 & $\Phi 1 \mathrm{X}$ & $\Phi 2 \mathrm{XX}$ & Ф3 $\mathrm{X}$ \\
\hline Class 3 & $\Phi 1 \mathrm{XX}$ & $\Phi 2 \mathrm{XXX}$ & (Ф3 XX \\
\hline Class 4 & Ф1 XXX & $\triangle 2 X X$ & Ф3 XX \\
\hline
\end{tabular}

Figure 3: Composition of nuclear export signal consensus sequences. $\Phi$ is Leu, Val, lle, Phe or Met; $\mathrm{X}$ is any amino acid. 
Crystals structures of several NESes bound to CRM1 demonstrated that the hydrophobic residues of the NES bind CRM1 specifically in 5 corresponding hydrophobic pockets (Ф0 - Ф4). The structural studies revealed the pocket $\Phi 0$ is less restricted to hydrophobic residues compared to the other pockets (Fung et al. 2015; Fung, Fu, and Chook 2017).

\subsubsection{The overall structural arrangement of CRM1 - cargo interactions}

The crystal structure of the part composed of the amino acids $707-1027$ of human CRM1 was published in 2004 (PDB ID: 1W9C) and revealed the HEAT repeats - helices architecture of CRM1 (Figure 4) (Petosa et al. 2004). In 2009 two crystals structures of full length CRM1 were published in complex with SPN1 (CRM1-SPN1, PDB ID: 3GB8) as well as in complex with SPN1 and RanGTP (CRM1-RanGTP-SPN1, PDB ID: 3GJX) (Dong et al. 2009; Monecke et al. 2009). The crystals structures present CRM1 as a ring-shaped protein consisting of 21 tandem HEAT repeats, where an outer convex surface is formed by A-helices and an inner concave surface is formed by $B$ helices (Figure 4). Besides the helical structural elements of CRM1, HEAT9 loop is extended into a stretch of acidic residues that from a $\beta$-hairpin termed the acidic loop (Figure 4) (Monecke et al. 2009). RanGTP is bound to CRM1 with the first 3 HEAT repeats of the N-terminal region which has been termed as the CRIME domain (CRM1, importin $\beta$, etc.) (Petosa et al. 2004). This region demonstrates the highest degree of sequence conservation between CRM1 and other receptors in the importin $\beta$ superfamily which further indicates for its functional importance (Görlich et al. 1997; Monecke et al. 2014). Whereas, the NES peptide binds in the hydrophobic cleft formed between the HEAT helices $11 \mathrm{~A}$ and $12 \mathrm{~A}$ (NES-binding cleft) (Figure 4). The NES-binding cleft contains 5 fixed hydrophobic pockets that represent a docking station for the critical hydrophobic residues of the NES peptide. The type, size, and the position of the key hydrophobic residues are crucial determinants of NES - CRM1 binding affinity. It has been shown that changing a singly key residue to a polar residue leads to a significantly weaker NES-binding (Dong et al. 2009). Furthermore, it was observed that the removal of a hydrophobic residue, for example the first methionine of SPN1 (Ф0), abolishes SPN1 binding to CRM1 (Monecke et al. 2009). This further reflect the importance of the specific docking of the key hydrophobic residues in the corresponding pockets for cargo binding. Structural analysis of several NESes bound to CRM1 showed that the different spacings in individual NESes are compensated by different structural arrangements of the NES peptide, which enables the proper alignment of the hydrophobic resides and their corresponding binding pockets (Fung et al. 2015, 2017; Güttler et al. 2010). 
A

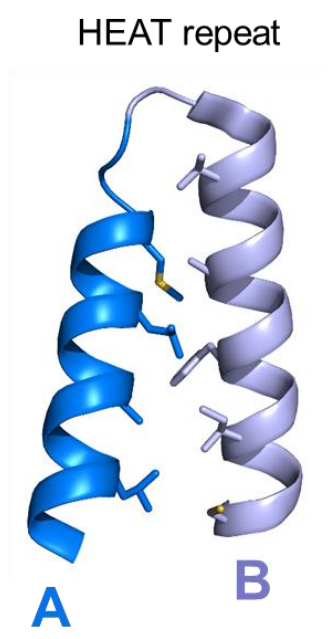

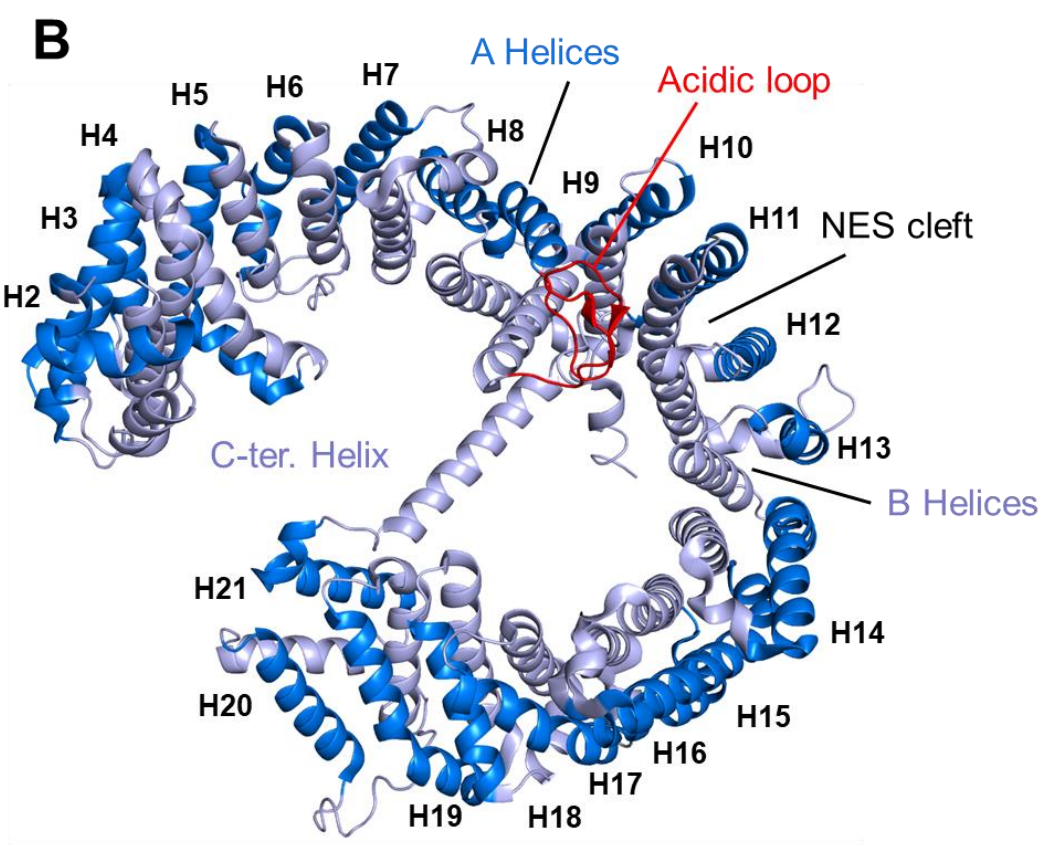

Figure 4: Overall architecture and structural arrangement of CRM1. (A) a general structure of a HEAT repeat demonstrates two antiparallel helices (helix $A$ and helix $B$ ) connected by a short linker loop. The HEAT repeat is stabilized by pronounced hydrophobic interactions (depicted as sticks) between helices A and B. (B) an overall CRM1 structure depicted in cartoon representation. CRM1 has an overall superhelical conformation that is composed by the stacking of 21 tandem HEAT repeats (H1$\mathrm{H} 21$ ). A-helices (marine) form the outer convex surface, while B-helices (lightblue) form the inner hydrophobic core of CRM1. The NES-binding cleft is located between HEAT repeats 11 and 12 . The acidic loop (red) is a $\beta$-hairpin formed by a stretch of acidic residues at HEAT9 loop.

CRM1 structures in complex with SPN1 unveiled it as unique CRM1 cargo with an exceptional binding mode. SPN1 is an import adaptor for uridine-rich small nuclear RNP (UsnRNP); it interacts with $\mathrm{m}^{3} \mathrm{G}$-cap of the UsnRNP and thereby enhances their $\mathrm{m3G}$-cap dependent nuclear import (Huber et al. 1998). Once the import is achieved, SPN1 is re-localized by CRM1-mediated nuclear export into the cytoplasm (Paraskeva et al. 2000). SPN1 has an N-terminal NES that contains 5 hydrophobic binding residues (Monecke et al. 2009). Unlike most nuclear export cargos SPN1 can form a complex with RanGTP in the absence of RanGTP which indicates exceptional binding properties (Dong et al. 2009). Crystal structures revealed that SPN1 - CRM1 interactions are not only mediated by the NES, it rather involves several regions in both proteins. The cap binding domain (CBD) of SPN1 comprising the residues 97-300 interacts with HEAT repeats 12-14' A helices. In addition, the C-terminal residues 349-360 of SPN1 interacts with A helices of HEAT repeats 14-16 (Dong et al. 2009; Monecke et al. 2009). The multipartite binding mode of SPN1 results in an exceptionally stable binary export complex even in the absence of RanGTP. 
Another unique feature of CRM1 that was unveiled by the SPN1 complex structures in the presence and the absence of RanGTP is the orientation of the HEAT repeat helix $21 \mathrm{~B}$. In the presence of RanGTP the helix 21B (C-terminal helix) arranges in a parallel orientation to helix 21A, while in the absence of RanGTP the helix 21B is bridging the central opening of CRM1 and interact with the lower part of HEAT repeats forming the NES-binding cleft (Dong et al. 2009; Monecke et al. 2009). The different conformations of the C-terminal helix indicated the high structural flexibility of CRM1 and presented the first clue towards the understanding of the allosteric interaction between RanGTP and the cargo protein (Monecke et al. 2014).

\subsubsection{Structural and conformational flexibility of CRM1}

CRM1 transport cycle follows the general cycle of nuclear export as described in Figure 2. Unlike SPN1, most cargoes need to bind CRM1 cooperatively in the presence of RanGTP to form a stable export complex. After the complex is assembled, it passes through the central channel of the NPC to the cytoplasm, where RanBP1 and RanBP2 together with the activity of RanGAP lead to the hydrolysis of Ran nucleotide and the dissociation of the export complex (reviewed in (Monecke et al. 2014)). Structural analysis of CRM1 during different stages of the export cycle revealed that CRM1 demonstrates high structural flexibility. Furthermore, the detailed structural investigation of unliganded CRM1 (PDB ID: 4FGV), the export complex CRM1-RanGTP-SPN1 (PDB ID: 3GJX), and the disassembly complex CRM1-RanGTP-RanBP1 (PDB ID: 3M1I) determined the acidic loop, Cterminal helix, and the NES-binding cleft as the key structural elements of CRM1 functionality and structural flexibility (Koyama and Matsuura 2010; Monecke et al. 2009, 2013). CRM1 is represented in two main conformations in crystal structures: an extended and pitched superhelical conformation as well as a more compact toroidal conformation (Figure 5). The extended conformation is displayed by the crystal structure of unliganded CRM1 from Chaetomium thermophilum (PDB ID: 4FGV). This conformation lacks the interaction between the $\mathrm{N}$ - and the $\mathrm{C}$ - terminal regions. Furthermore, in this conformation the acidic loop adopts a so called "flipped back" position where it interacts with the backside of the NES-binding cleft, whereas the C-terminal helix demonstrates a crossing position as it spans the core of CRM1 and interact with the base of helix B of HEAT repeat 12 (Figure 5). These structural arrangements lead to a narrowed (closed) NES-binding cleft that is not accessible by the NES peptide (Monecke et al. 2013). On the other hand, the compact conformation is stabilized upon the formation of the ternary export complex and characterized by the tight interactions between the $\mathrm{N}$ - and $\mathrm{C}$ terminal regions (Figure 5). In this conformation the RanGTP binding triggers the rearrangement of the C-terminal helix in a parallel position to helix 21A. Whereas the acidic loop re-arranges to 
a "seat belt" like conformation as it detaches from the back of the NES and wrap around Ran providing further stability for Ran binding (Monecke et al. 2009, 2014). The overall structural rearrangement from the extended to the compact conformation leads to the opening of the NES binding cleft and allows that cargo NES to bind to CRM1 (Figure 5). Taken together, performed structural analysis indicates that the cooperativity of RanGTP and cargo interactions is achieved by the structural arrangement of the acidic loop and the C-terminal helix and its coupling to the NES-binding cleft (Dölker et al. 2013; Koyama and Matsuura 2010; Monecke et al. 2014).

overall conformation

Extended CRM1
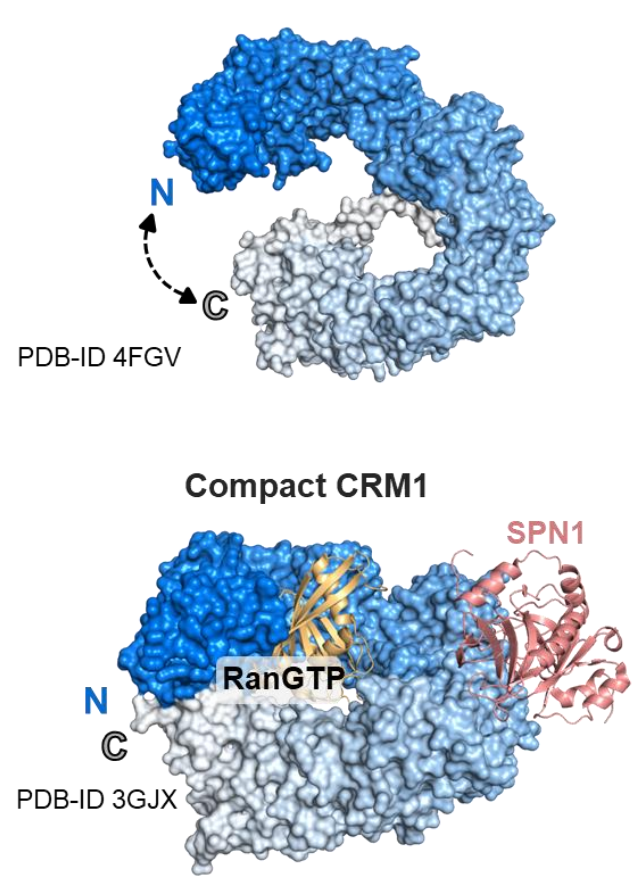

structural details
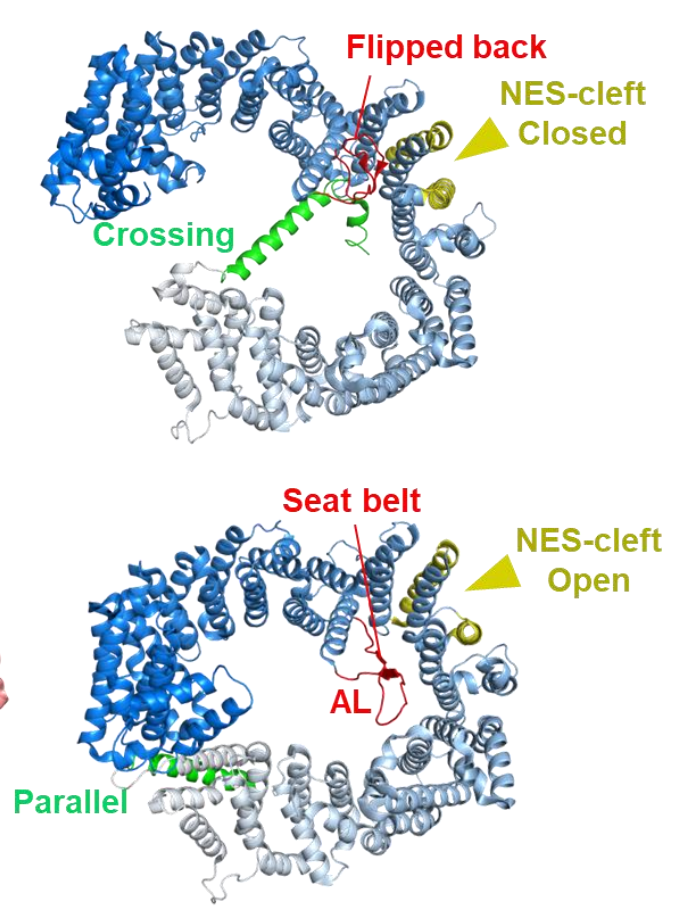

NES- binding cleft
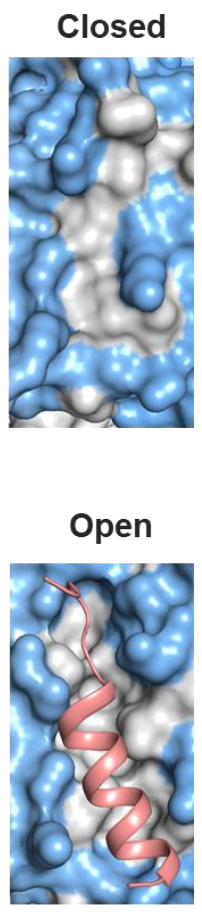

Figure 5: Structural and conformational flexibility of CRM1 during the export cycle. Left, surface representation of CRM1 gradient colored from $\mathrm{N}$-terminus (marine) to the $\mathrm{C}$ terminus (white) in the unliganded state and in the export complex CRM1-RanGTP-SPN1. Unliganded CRM1 exhibits an extended superhelical conformation that lacks the interactions between the $\mathrm{N}$ - and $\mathrm{C}$ - termini. In the export complex CRM1 adopts a more compact-toroidal conformation that involves tight interactions between the $\mathrm{N}$ - and $\mathrm{C}$ - terminal regions. Middle, CRM1 in cartoon representations show the structural arrangement of the acidic loop (red) and the C-terminal helix (green) in the corresponding conformation. Right, a surface representation of the NES-binding cleft topography in different conformations (hydrophobic resides are shown in grey). The structural arrangements of the extended conformations lead to a constricted and a closed cleft. The overall conformational transition into the compact conformation leads to an open cleft that can bind the NES peptide (SPN1-NES depicted as cartoon). 
Further structural analysis of Chaetomium thermophilum CRM1 ( ${ }^{C t}$ CRM1) by single particle electron microscopy (EM) revealed that free CRM1 (in the absence of cargo and RanGTP) samples at equilibrium in both conformations that are displayed in different crystal structure. Two third of ${ }^{C t}$ CRM1 particles classified by single particle EM adopted the extended superhelical conformation, while the remaining third depicted the more compact conformation that is observed in export complex structure (Monecke et al. 2013). Furthermore, integrative structural analysis of human CRM1 ( $\left.{ }^{H s} \mathrm{CRM} 1\right)$ by single particle EM together with small-angle X-ray scattering (SAXS) and molecular dynamics (MD) simulations revealed an even more pronounced conformational flexibility in mammalian CRM1 (Dölker et al. 2013). Interestingly, in contrast to ${ }^{C t} \mathrm{CRM} 1$, single particle EM showed that half of ${ }^{H s} \mathrm{CRM} 1$ particles adopt the extended conformation. Whereas, the remaining particles resemble several intermediate conformers of more compact conformations that were classified into several subpopulations (Dölker et al. 2013). This indicates the high degree of ${ }^{H s}$ CRM 1 conformational flexibility in solution. Further investigations by $\mathrm{MD}$ simulations on both ${ }^{\mathrm{Ct}} \mathrm{CRM} 1$ and ${ }^{\mathrm{Hs}} \mathrm{CRM} 1$ wild type proteins and in silico acidic loop and $\mathrm{C}$-terminal helix truncated variant indicated for the crucial effect of both elements on CRM1 conformational flexibility and its relation to cargo and Ran binding (Dölker et al. 2013; Monecke et al. 2013).

\subsubsection{CRM1 interaction with the nuclear pore complex}

The FG-repeat barrier in the central channel of the NPC prevents most proteins to pass through the NPC. However, the unique composition and structural architecture of importin $\beta$ superfamily transport receptors permit their transfer through the FG-repeats barrier across the NPC. In order to understand the interactions of nuclear transport receptor with the FG-repeats at an atomic level, several receptors were crystallized with NUP fragments containing FG-repeat motifs (Bayliss et al. 2002; Bayliss, Littlewood, and Stewart 2000; Grant, Neuhaus, and Stewart 2003; Koyama and Matsuura 2010; Liu and Stewart 2005; Matsuura and Stewart 2005; Pumroy et al. 2012). FG repeats are classified into four major motifs: FxFG, GLFG, SxFG and PxFG, where $x$ is any amino acids. Within the FG-NUPs, these motifs are usually separated by a linker of variable length with a typical spacing of around 20 amino acids (Aramburu and Lemke 2017; Denning et al. 2003; Dölker, Zachariae, and Grubmüller 2010). Importin $\beta$ is one of the best studies transport receptors with respect to interaction with FG-NUPs. Human importin $\beta$ (residues 1-442) was successfully crystalized with FG-repeats of different motifs including GLFG and FXFG. Crystal structures showed the FG motifs bind importin $\beta$ by the hydrophobic interactions at the outer surface of the N-terminal region (Bayliss et al. 2002, 2000; Liu and Stewart 2005). Another 
structure identified a different binding site at the $\mathrm{C}$-terminal half of importin $\beta$. Further analysis by MD simulations predicted Importin $\beta$ (and most likely other transport receptors) contains several addition FG-NUPs binding site (Isgro and Schulten 2005). This indicated that surface of transport receptors retains multiple FG binding sites that facilitate the passage of the transport receptors through the central channel of the NPC by binding to FG motifs of different FG-NUPs.

Recently, the crystal structure of an FG-repeat-containing fragment of Nup214, in complex with human CRM1, Snurportin1, and RanGTP unveiled CRM1 has multiple FG-repeat' interacting regions with several FG-binding sites (Figure 6) (PDB ID: 5DIS) (Port et al. 2015). The 117 amino acids-fragment contained seven characteristic FG motifs and a similar FS motif (F1-F8). The structure showed the FG motifs are the prominent anchor points of the FG-repeats containing fragment that wraps around the outer convex surface of CRM1 (Figure 6). Unlike the FG-motifs, the spacing sequences were loosely attached to CRM1 and therefore were not entirely modeled in the structure. Three different FG-binding patches were identified for the binding of 8 FG motifs in corresponding binding pockets (P1-P8). The first binding region was identified the surface of HEAT repeats 14-19 and it contains the FG motifs F1-F4. The second binding region, located at the C-terminal end of CRM1 involves HEAT repeats $17-20$ and contains 2 FG-motifs F5 and F6, while the third region was located at the $\mathrm{N}$-terminal HEAT repeats 2-4 and contains the remaining FG-motifs F7 and F8 (Figure 6). The observed binding mode of the FG-repeats-containing fragment connecting the $\mathrm{N}$ - and $\mathrm{C}$ - terminal regions renders Nup214 a molecular clamp that stabilizes the compact conformation of CRM1 export complex. The multiple binding sites for FGmotifs distributed at the outer surface of CRM1 contribute to higher avidity interaction between CRM1 and FG-repeats. On the other hand, the flexible and loose binding of the linker regions between FG-motifs contribute to weak interaction avidity (Port et al. 2015). Such a binding mode is important to maintain interactions that are strong enough to achieve the association of the transport receptors to the NPC, yet not too strong so that it allows the translocation of the receptor through the NPC.

In 2017, another structure of the yeast Saccharomyces cerevisiae CRM1 ( $\left.{ }^{5 c} C R M 1\right)$ was published in complex with SxFG/PxFG repeat peptide of the yeast Nup42 (PDB ID: 5XOJ) (Koyama et al. 2017). The structure unveiled three binding regions for FGxF/PxFG motifs at the outer surface of the C-terminal third of CRM1 including the HEAT repeats 14-20. The identified binding regions retained a total of five hydrophobic pockets for the binding of phenylalanine side chains. Four of the identified pockets were identical to binding pockets of FG-repeat motifs in the binding regions 1 and 2 of nup214 FG-repeat fragment. Similar to Nup214 interactions, the multiple binding site 
of Nup42 FG-repeats was observed to increase the binding affinity to CRM1. On the other hand, it was shown that the binding of yeast RanBP1 weakens CRM1 interaction with Nup42 FGxF/PxFG motifs, which suggested the initiation of the export complex dissociation at the cytoplasmic side of the NPC. Furthermore, unliganded CRM1 demonstrated a much weaker binding to Nup42 FGxF/PxFG motifs. This led to the conclusion that transient binding of the SxFG/Px FG-Nups to unliganded CRM1 would facilitate its rapid recycling back to the nucleus (Koyama et al. 2017).

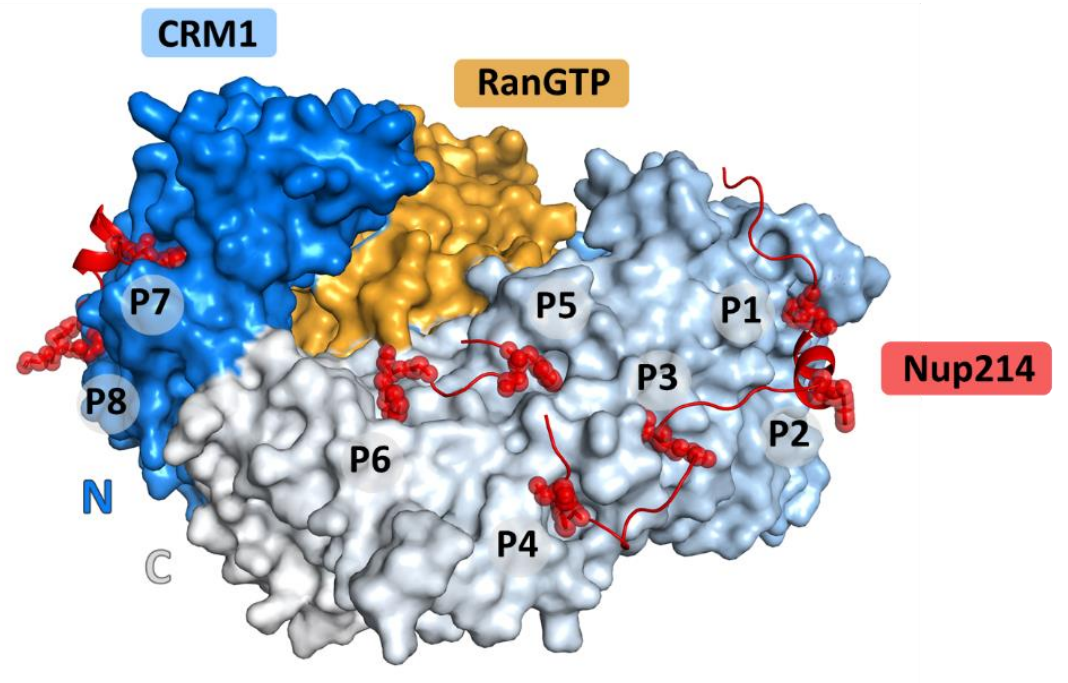

Figure 6: Overall structure of Nup214 FG-repeat fragment bound to CRM1-RanGTP-SPN1 complex (PDB ID: 5DIS). CRM1 and Ran are depicted in surface representation. CRM1 is gradient colored from the $\mathrm{N}$-terminus (marine) to the $\mathrm{C}$ - terminus (white), while Ran is colored lightorange. Nup214 FG-repeat fragment, shown in cartoon representation (red), wraps around the outer surface of CRM1 via the FG motifs (depicted in spheres) as the main anchor points. FG motifs bind CRM1 in specific corresponding pockets (P1-P8) in 3 identified regions. The first and the second regions are located at the C-terminal part of CRM1 and contain the binding pockets (P1-P4) and (P5-P6) respectively. The third binding region is located at the $\mathrm{N}$-terminal side of CRM1 and it contains the binding pockets P7 and P8. SPN1 is not shown in the figure.

\subsection{Altered nucleocytoplasmic distribution of proteins in cancer}

The appropriate nucleocytoplasmic distribution of proteins and RNP complexes is crucial for maintaining the normal functionality of mammalian cells. Mislocalization of proteins have pathological consequences as it disturbs the physiological nucleus/cytoplasmic homeostasis and interferes with critical regulatory pathways. In different cancer cells, a large variety of oncoproteins and tumor suppressor proteins including the nuclear factor of activated T-cells (NFAT), adenomatous polyposis coli protein (APC), Survivin, p53, retinoblastoma protein (Rb), and Bcr-Abl are aberrantly mislocalized (Figure 7) (Faustino et al. 2007; Hill et al. 2014; Turner, 
Dawson, and Sullivan 2012). The mislocalization of these proteins results in the inactivation/activation of critical regulatory pathways and interferes with cell cycle regulators, cellular proliferation, and apoptosis which leads to caner initiation and progression. For example, in normal cells the transcription factor p53, which is well known for its vital role in genome protection, is localized in the nucleus (Levine and Greenbaum 2012). In cancer cells, p53 is deactivated by blocking its ability to bind to DNA which is achieved either by several mutations or by the abnormal cytoplasmic localization of the wild type protein (Hollstein et al. 1991; Lu et al. 2000; Tweddle et al. 2001). Cytoplasmic localization of p53 has been observed in several cancer types (including, colon, breast, retinoblastoma, and ovaria cancer) and its usually mediated by the unmasking of an NES coupled with its active nuclear export (Foo et al. 2007; Stommel et al. 1999). Another similar example is $\mathrm{Rb}$ (Retinoblastoma susceptibility gene product) which is a tumor suppressor protein that plays a crucial role in regulating the cell cycle G1-S phase transition (Burkhart and Sage 2008). Normally, Rb is localized in the nucleus, while its cytoplasmic mislocalization has been observed in specific cancers (Jiao et al. 2006; Kowalik et al. 2013; Storke et al. 1993). Inactivation of Rb mediated by aberrant nucleocytoplasmic transport alters normal cell proliferation and thereby accelerates cancer initiation. It furthermore leads to mitotic fidelity and genome instability which renders $\mathrm{Rb}$ a vital factor in several cancer diseases (Burkhart and Sage 2008; Mittnacht 1998; Zamanian and La Thangue 1993).

Other groups of tumor suppressor proteins like the APC protein are distributed in both compartments in normal cells but shifted towards either the nucleus or the cytoplasm in several cancer diseases (Figure 7). APC protein contain an NES and an NLS that allow it to shuttle between both compartments as it regulates several cellular functions (Kristi L. Neufeld et al. 2000; Neufeld and White 1997). One of the crucial functions of APC is the degradation of $\beta$ catenin which is a key component of the Wnt signaling pathway (K L Neufeld et al. 2000). In the absence of Wnt signaling low levels of $\beta$-catenin are maintained by the activity of APC together with glycogen synthase kinase 3 beta (GSK-3ß) and Axin (Cong and Varmus 2004; Zeng et al. 2008). In cancer cells the inactivation of APC results in the nuclear accumulation of $\beta$-catenin, which was highly correlated to colon cancer initiation. In this sense, a C-terminally truncated version of APC was identified in $60 \%$ of tested colon cancer patients (Powell et al. 1992). The truncated version of the protein accumulates in the nucleus leading to the deregulation of $\beta$ catenin degradation. The consequent accumulation of $\beta$-catenin in the nucleus leads to cancer initiation by interfering with gene expression and initiating gene transactivation (Henderson and Fagotto 2002; Jamieson, Sharma, and Henderson 2014). 


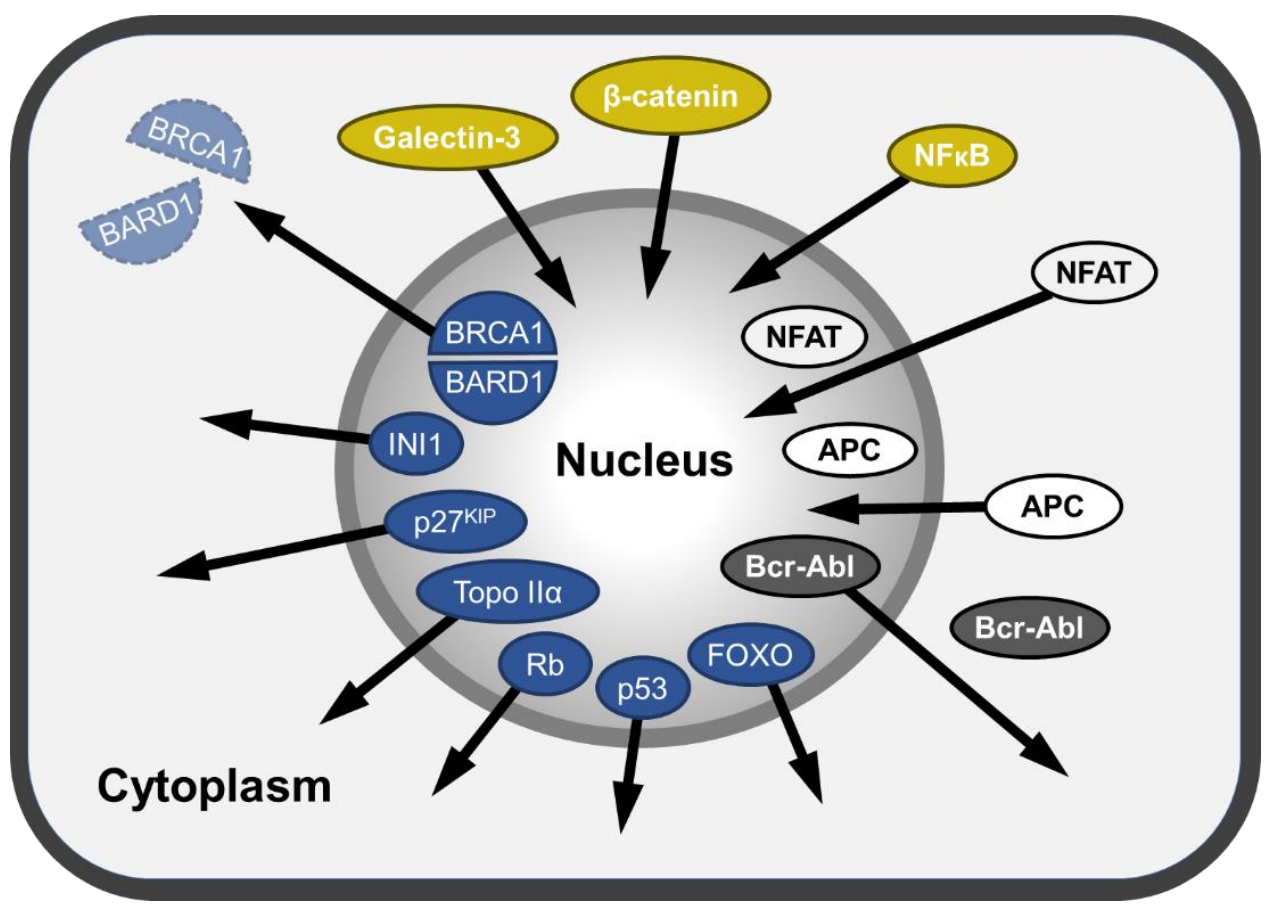

Figure 7: Altered subcellular localization of tumor suppressor proteins and oncoproteins in cancer cells compared to normal cells. In cancer cells several nuclear proteins are mislocalized in the cytoplasm (highlighted in blue), whereas several cytoplasmic proteins are mislocalized in the nucleus (highlighted in yellow). Other proteins are distributed in both compartments in normal cells, but shifter toward either the nucleus (highlighted in white) or the cytoplasm (highlighted in grey) in cancer cell. The direction of shift in cancer cells is indicated by arrows. The figure is modified from (Dickmanns et al. 2015).

Similar to APC, the transcription factor family NFAT can be localized in both the nucleus and the cytoplasm in normal cells (Beals et al. 1997; Kehlenbach et al. 1998). The NFAT transcription factors act as calcium sensors and regulate the expression of a multitude of genes that are essential for vertebrate development as well as several cellular functions including cell proliferation, migration, invasion, and angiogenesis (Crabtree and Olson 2002; Müller and Rao 2010). Upon stimulation NFAT is activated by dephosphorylation, which is attained by calcium activated calcineurin (serine/threonine phosphatase). Following the dephosphorylation lysinerich NLS is exposed enabling the nuclear import of the transcription factors (Rao, Luo, and Hogan 1997). In the resting state, the phosphorylation of NFAT by GSK-3 $\beta$ exposes an NES shuttling the proteins back to the cytoplasm (Gwack et al. 2006; Kehlenbach et al. 1998). In several tumors and hematological malignancies, the overexpression and/or constitutive activation of NFAT isoforms has been reported. This leads to the nuclear accumulation of the transcription factors resulting in the downstream activation of their target genes (Mancini and Toker 2009; Pan, Xiong, and Chen 2013; Qin et al. 2014). 
The mislocalization of the above-mentioned examples as well as several other critical tumor related factors is dependent on the nucleocytoplasmic transport machinery of the cell. Meaning that the prerequisite mislocalization of the oncoproteins and tumor suppressors leading to cancer initiation and progression requires the importin $\beta$ transport receptors, among which CRM1 demonstrates the highest versatility (Dickmanns et al. 2015; Faustino et al. 2007; Sun et al. 2016).

\subsubsection{CRM1 in cancer}

CRM1 was identified as the nuclear export receptor that mediates the mislocalization of the previously mentioned examples: p53 (Foo et al. 2007), Rb (Jiao et al. 2008), and APC (Henderson 2000) as well as other several proto-oncoproteins and tumor suppressor proteins including p21 ${ }^{\mathrm{CIP}}$ (Hwang, Kim, and Kwon 2007), cyclin D1 (Benzeno and Diehl 2004), BRCA1 (Rodríguez and Henderson 2000), Bok (Bartholomeusz et al. 2006), forkhead box (FoxO) proteins (Howell and Stoffel 2009; De Laté et al. 2010), N-WASP (Suetsugu and Takenawa 2003), nucleophosmin (Wang et al. 2005), and p27 KIP (Ishida et al. 2002) (Figure 7). The aberrant localization of such proteins can be caused by mutations or modifications that interfere with their transport activities such as the unmasking of a normally hidden NES (e.g. p53 , BRCA2/RAD51, NFAT, INI1) (Craig et al. 2002; Jeyasekharan et al. 2013; Stommel et al. 1999; Zhu and McKeon 1999), phosphorylation (e.g. p27, Rb, p53) (Ishida et al. 2002; Jiao et al. 2006; Zhang and Xiong 2001), ubiquitination (e.g. p53) (Lohrum et al. 2001), or SUMOylation (e.g. p53) (Santiago et al. 2013). Another main factor that contributes to the aberrant localization of these proteins is the change of CRM1 cellular levels (Watt and Leaner 2010). Elevated CRM1 expression has been reported in several cancers and is correlated with poor patient prognosis in patients with ovarian- (Noske et al. 2008), cervical- (Van Der Watt et al. 2009), pancreatic- (Huang et al. 2009), kidney- (Inoue et al. 2013), and breast cancers (Yue et al. 2018) as well as osteosarcoma (Yao et al. 2009), glioma (Shen et al. 2009), gastric carcinomas (Zhou et al. 2013), and leukemia (Kojima et al. 2013). Furthermore, CRM1 elevated expression has been accompanied with mantle cell lymphoma (Zhang et al. 2013), multiple myeloma (Schmidt et al. 2013), and melanoma (Pathria, Wagner, and Wagner 2012).

A prominent example of tumor suppressors mislocalized by CRM1 is the cyclin-dependent kinase inhibitor $\mathrm{p} 27^{\mathrm{KIP}}$. In normal cells $\mathrm{p} 27^{\mathrm{KIP}}$ is localized in the nucleus, where it blocks cell cycle progression at the G1 phase by binding and inhibiting cyclin/cyclin-dependent kinase (CDK) complexes (Sherr and Roberts 1999). The interaction of p27 $7^{\mathrm{KIP}}$ with CDK complexes inhibits their kinase activity and by that prevents the phosphorylation and subsequent inactivation of the 
critical transcriptional repressor $\mathrm{Rb}$ (Alexander and Hinds 2001). The cytoplasmic mislocalization of $\mathrm{p} 27^{\mathrm{KIP}}$ mediated by CRM1 is dependent on the phosphorylation of its serine 10 (Ishida et al. 2002). Following this modification $\mathrm{p} 27^{\mathrm{KIP}}$ is exported by CRM1 to the cytoplasm, where it is subjected to proteasomal degradation or functions as an oncogene by promoting cell migration (Connor et al. 2003; Wander, Zhao, and Slingerland 2011). The aberrant cytoplasmic mislocalization of $\mathrm{p} 27^{\mathrm{KIP}}$ leads to the phosphorylation and inactivation of $\mathrm{Rb}$, resulting in the abnormal expression of multiple factors and subsequent promotion of uncontrolled cell cycle progression (Besson, Assoian, and Roberts 2004; Sherr and Roberts 1999; Wang et al. 2014). High levels of serine 10 - phosphorylated p27 $7^{\mathrm{KIP}}$ have been reported in breast cancer patients and correlated with high tumor grade and poor clinical prognosis (Fagan-Solis et al. 2014; Song et al. 2015).

Subcellular mislocalization of other oncoproteins can be initiated by the masking/unmasking of their NES/NLS signal peptides. For example, the breast cancer susceptibility protein 1 (BRCA1) is actively imported to the nucleus as it retains an NLS (Thakur et al. 1997). In the nucleus BRCA1 forms a stable heterodimer with BRCA1-associated RING domain protein 1 (BARD1) (Baer and Ludwig 2002). The formed heterodimer is involved in several cellular functions like DNA repair and interact with cell cycle regulators (Schüchner et al. 2005; Scully et al. 1997). It turned out that both BRCA1 and BARD1 are subjected to CRM1-mediated nuclear export as they bear NESes (Rodriguez et al. 2004; Rodríguez and Henderson 2000). However, both proteins NESes are present at the dimerization surface and therefore they are masked when both proteins form a dimer (Jeyasekharan et al. 2013; Rodriguez et al. 2004). On the other hand, in cancer cells, the disruption of BARD1/BRCA1 dimerization exposes their NESes which triggers their nuclear export in CRM1-dependent fashion. The nuclear export of both proteins coupled with an impaired nuclear import in cancer cells results in their cytoplasmic accumulation which promotes breast cancer metastasis and cancer development (Mahmoud et al. 2017; Santivasi et al. 2015).

Besides its critical role in cancer initiation and progression, CRM1-dependent export was found to interfere with cancer treatment as it mediates the cytoplasmic mislocalization of wellestablished drug targets like Topoisomerase Il $\alpha$ (Turner et al. 2012). Topoisomerase Il $\alpha$ is a nuclear protein that plays an essential role in DNA replication and involved in transcription, chromatid separation, and chromatin condensation (Nitiss 2009a; Wang 2002). In multiple myeloma topoisomerase $I 1 \alpha$ is specifically targeted by doxorubicin and etoposide that act on DNA-bound nuclear topoisomerase ll $\alpha$ (Nitiss 2009b). However, in myeloma and at an increased cell densities, topoisomerase Il $\alpha$ was observed to be exported to the cytoplasm in CRM1- 
dependent manner, which rendered the cells resistant to topoisomerase Il $\alpha$-targeting drugs (Engel et al. 2004; Valkov and Sullivan 1997). CRM1 interference with cancer treatment has been reported for other drug targets like Galectin-3 and BCR-ABL (reviewed in (Turner et al. 2012)).

\subsection{CRM1 in viral diseases}

Besides its significant contribution in cancer diseases, CRM1 is utilized by viruses from different families including coronaviruses, orthomyxoviruses, paramyxoviruses, flaviviruses, retroviruses, rhabdoviruses, and herpesviruses for the establishment of viral infections. Several viruses belonging to these families have been shown to utilize CRM1-mediated export during different stages of the virus replication cycle, mainly for the nuclear export of the viral RNAs and RNP complexes (Cao et al. 2012; Cao and Liu 2007; Elton et al. 2001; Ghildyal et al. 2009; Nakano and Watanabe 2016; Pasdeloup et al. 2005; Sanchez et al. 2007). The key role of CRM1 in several viral infections has been indicated by the early discovery of an NES in the HIV-1-Rev protein (Fischer et al. 1995). The viral protein HIV-1-Rev is a phosphoprotein with a molecular weight of $19 \mathrm{kDa}$. In the infected cells, HIV-1-Rev is localized in the nucleus by an NLS, where it mediates the controlled expression of retroviral mRNAs. In addition, the HIV-1-Rev contains an RNA binding domain known as Rev response element (RRE)-binding domain as well as an activation domain that harbors a leucin-rich NES. It was shown that HIV1-Rev utilizes the CRM1-depedent export for the shuttling of unspliced viral RNA into the cytoplasm (Fontoura, Faria, and Nussenzveig 2005; Mathew and Ghildyal 2017; Najera, Krieg, and Karn 1999). The disruption of CRM1-Rev interaction by CRM1 inhibitors was observed to arrest the transcription of HIV-1 and thereby inhibits the production of new virions resulting in the reduction of HIV-1 levels (Daelemans et al. 2002; Fleta-Soriano et al. 2014; Perwitasari et al. 2014).

CRM1 has also been shown to have a key role in influenza viruses' replication cycle. Influenza are single stranded and segmented RNA viruses with a prototypical replication cycle that consists of viral attachment, endocytosis, and the release of viral RNP in the cytoplasm followed by its nuclear import, where it undergoes transcription and replication. Afterwards, newly formed viral RNPs are exported to the cytoplasm for the assembly of new viral particles (Dou et al. 2018). Several studies revealed that the nuclear export of influenza's viral RNPs is accomplished by an NES-containing nuclear export viral protein (NEP) that utilizes CRM1-depedent pathway. CRM1depedent export of viral RNP to the assembly site is a very critical step in the viral replication cycle and therefore is conserved in influenza strains A, B, and C (Elton et al. 2001; Paragas et al. 2001). The blockage of CRM1 mediated export of viral RNP results in their nuclear retention 
which lead to reduced viral replication (Chutiwitoonchai et al. 2017; Perwitasari et al. 2014; Watanabe et al. 2001).

In addition to HIV and influenza, CRM1 is a critical factor in several other viral infections including human T-cell leukemia virus type-1, respiratory syncytial virus, dengue virus, rabies virus, and human cytomegalovirus. Inhibiting CRM1-mediated export has been correlated with improved antiviral host immune responses, reduced infectivity, and incomplete viral assembly (reviewed in (Mathew and Ghildyal 2017)).

\subsection{Inhibition of CRM1-mediated nuclear export}

The significant role of CRM1 in the development of cancer and viral diseases as well as its interference with cancer treatment rendered it a particularly interesting target for therapeutic intervention in diverse cancer types and viral diseases. Initial investigations in several cancer researchers revealed that inhibiting CRM1 counteracts the subcellular mislocalization of several (including previously mentioned) oncoproteins and tumor suppressor proteins, suggesting that CRM1 inhibition can represent a valid strategy for cancer treatment (Dickmanns et al. 2015; Hill et al. 2014; Sun et al. 2016). For example, in several cancers CRM1 inhibition was shown to significantly increase p $27^{\mathrm{KIP}}$ levels and decreases the cytoplasmic levels of its oncogenic variant (serine 10- phosphorylated $\mathrm{p} 27^{\mathrm{KIP}}$ ); this was correlated with reduced tumor size and reduced tumor cell proliferation (Azmi et al. 2013; Zheng et al. 2014). Furthermore, subsequent experiments revealed that blocking CRM1-mediated export of topoisomerase Il $\alpha$ sensitizes multiple myeloma cells to doxorubicin and etoposide, suggesting CRM1 is a valid and significant target in combination therapy (Turner et al. 2009, 2013). Over the last two decades several natural and synthetic compounds were discovered or developed for the inhibition of CRM1mediated nuclear export. While most of these compounds were only tested in vitro or in mice, some have undergone further development as potential anti-tumor drugs and are already being evaluated in clinical trials (Mathew and Ghildyal 2017; Sendino, Omaetxebarria, and Rodríguez 2018). The underlying mechanism of nuclear export inhibition by all known compounds is based on the interference of CRM1-cargo interaction by the direct blockage of the NES-binding cleft (Sun et al. 2016). This section summarizes the most characterized and studied CRM1 inhibitors. A structural perspective on CRM1 inhibition will be described in the next chapter. 
Table 1: CRM1-targeting nuclear export inhibitors.

\begin{tabular}{|c|c|c|c|}
\hline Inhibitor & Source & Structure & References \\
\hline Leptomycin B & Streptomyces & & $\begin{array}{l}\text { (Kudo et al. } \\
\text { 1999; Sun et } \\
\text { al. 2013) }\end{array}$ \\
\hline Anguinomycin $\mathrm{A}$ & Streptomyces & & $\begin{array}{l}\text { (Bonazzi et } \\
\text { al. 2010; Sun } \\
\text { et al. 2013) }\end{array}$ \\
\hline Ratjadone A & $\begin{array}{l}\text { Sorangium } \\
\text { cellulosum }\end{array}$ & & $\begin{array}{l}\text { (Köster et al. } \\
\text { 2003; Sun et } \\
\text { al. 2013) }\end{array}$ \\
\hline KOS 2464 & $\begin{array}{l}\text { Semi } \\
\text { synthetic }\end{array}$ & & $\begin{array}{l}\text { (Mutka et al. } \\
\text { 2009; Turner } \\
\text { et al. 2012) }\end{array}$ \\
\hline Valtrate & $\begin{array}{l}\text { Valeriana } \\
\text { fauriei }\end{array}$ & & $\begin{array}{l}\text { (Tamura et } \\
\text { al. 2010) }\end{array}$ \\
\hline Curcumin & $\begin{array}{l}\text { Curcuma } \\
\text { longa }\end{array}$ & & $\begin{array}{l}\text { (Niu et al. } \\
2013 \text { ) }\end{array}$ \\
\hline
\end{tabular}


Table 1: continued

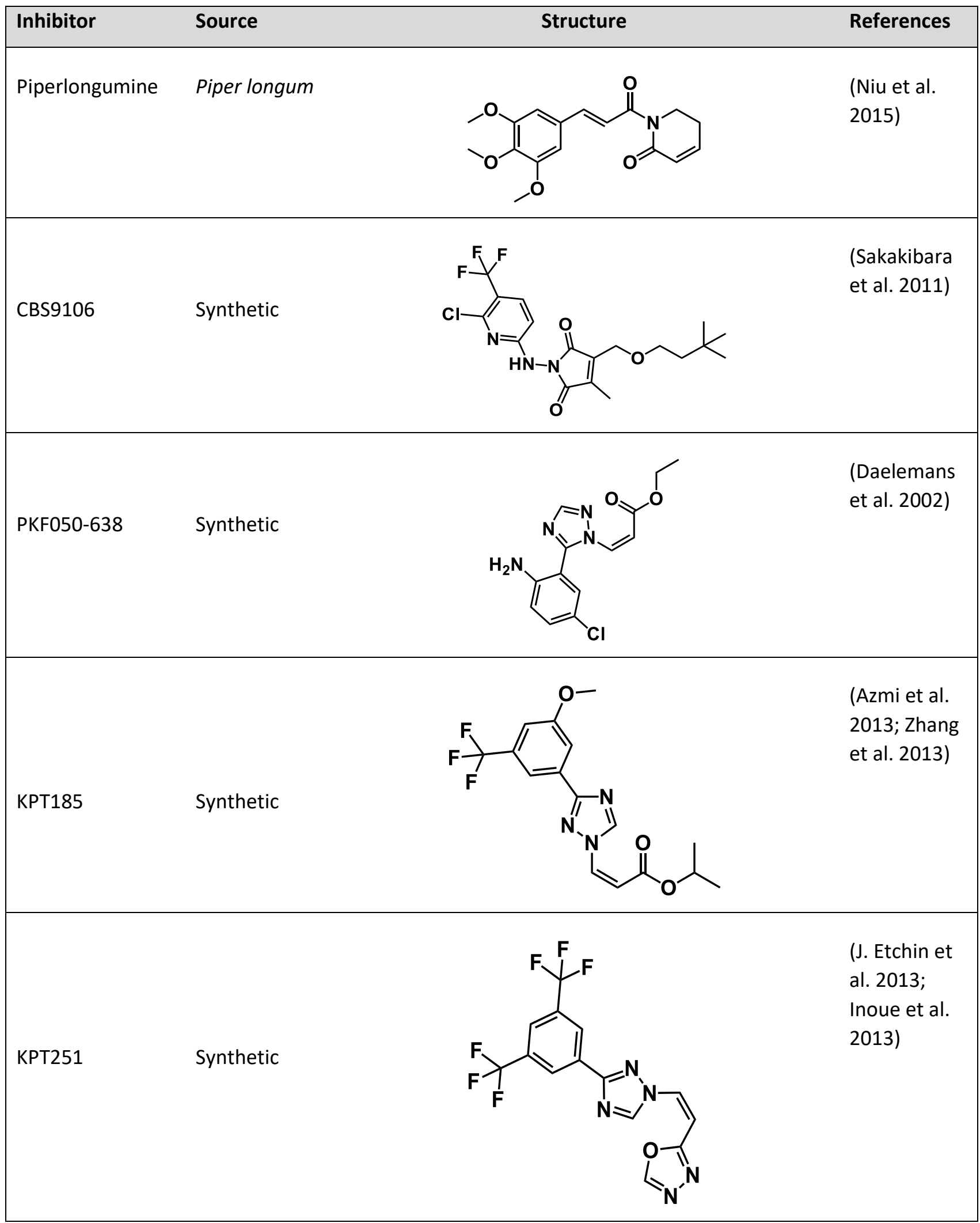


Table 1: continued

\begin{tabular}{|c|c|c|c|}
\hline Inhibitor & Source & Structure & References \\
\hline \multirow[b]{2}{*}{ КРТ276 } & & & $\begin{array}{l}\text { (Schmidt et } \\
\text { al. 2013; } \\
\text { Zhang et al. } \\
\text { 2013) }\end{array}$ \\
\hline & Synthetic & & \\
\hline $\begin{array}{l}\text { KPT330 } \\
\text { (Selinexor) }\end{array}$ & Synthetic & & $\begin{array}{l}\text { (Conforti et } \\
\text { al. 2015; } \\
\text { Julia Etchin } \\
\text { et al. 2013; } \\
\text { Syed 2019; } \\
\text { Zheng et al. } \\
\text { 2014) }\end{array}$ \\
\hline & & & $\begin{array}{l}\text { (Hing et al. } \\
2016 \text { ) }\end{array}$ \\
\hline КРТ8602 & Synthetic & & \\
\hline
\end{tabular}




\section{Leptomycin B}

Leptomycin B (LMB; also known as elactocin, mantuamycin, and NSC 364372) is a naturally produced compound by Streptomyces bacteria species and it is the first identified specific inhibitor of nuclear export (Hamamoto et al. 1983; Hamamoto, Uozumi, and Beppu 1985). It consists of an unsaturated, branched polyketide chain with a terminal $\alpha, \beta$-unsaturated d-lactone ring (Table 1). Initially LMB was discovered as an antifungal agent (Hamamoto et al. 1985). Nevertheless, it was found that it possesses an anti-tumor activity with a high potency against several experimental tumors including leukemia and lung carcinoma (Leopold et al. 1984; Roberts et al. 1986). A decade later, CRM1 was identified as the cellular target of LMB (Kudo et al. 1998; Nishi et al. 1994). Subsequent investigation in the Schizosaccharomyces pombe revealed that LMB covalently binds CRM1 at a specific cysteine residue (Cys529; equivalent to Cys528 in human) that is localized in the NES-binding cleft (Kudo et al. 1999). Recent structural analysis of LMB bound to genetically modified (Thr539Cys) CRM1 from Saccharomyces cerevisiae elucidated its conjunction mechanism by Michael-type addition (Sun et al. 2013). The reactivity of CRM1 towards LMB results in the hydrolysis of its lactone ring and its covalent bonding with the sulfur atom of the reactive cysteine. The stabilization of the hydrolyzed lactone ring by surrounding positively charged residues (mainly lysin and arginine) renders the binding irreversible (Sun et al. 2013).

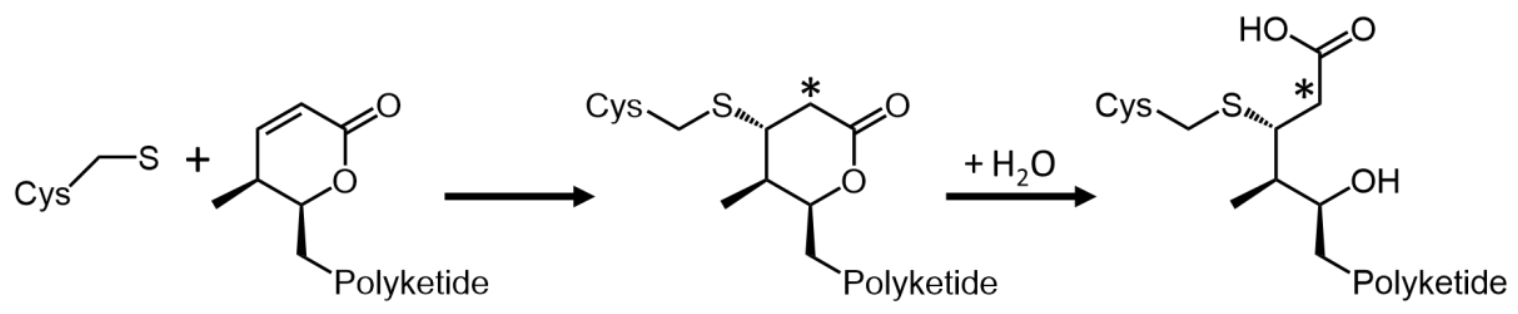

Figure 8: Covalent conjugation of Leptomycin B lactone ring with CRM1 reactive cysteine residue. The conjugation is mediated by Michael addition reaction and followed by the subsequent hydrolysis of the lactone ring. The positions of $\alpha$-protons of the Michael reaction sites are indicated by asterisks.

LMB demonstrated high potency an effective cell death against multiple cancer cell types in vitro. However, when it was tested clinically in a phase I trial it exhibited severe dose-limiting toxicities, including anorexia and malaise (Newlands, Rustin, and Brampton 1996). The toxicity of LMB triggered a persistent search and development for alternative CRM1-targeting compounds that could bind CRM1 in a similar fashion to LMB but display less toxicity and higher efficacy. To date, most CRM1 inhibitors function either by the permanent or the reversible modification of the 
reactive cysteine and thereby prevent the binding of the cargo's NES and subsequent formation of a stable export complex (Sun et al. 2016).

\section{LMB analogs: Ratjadone and Anguinomycins}

Ratjadones $A, B, C$, and $D$ are natural compounds with antifungal properties that are isolated from multiple strains of myxobacterium Sorangium cellulosum (Kalesse et al. 2001). Treatments with Ratjadone compounds at very low concentrations were shown to inhibit cell growth and proliferation in yeast, HeLa cells, and in different tumor cell lines (Burzlaff et al. 2003). Subsequent cell cycle analysis showed that Ratjadone compounds induce cell cycle arrest in the G1 phase (Burzlaff et al. 2003). Soon later it was revealed that Ratjadone A and the analogs B, C, and $D$ inhibit nuclear export by binding to CRM1 in the picomolar range, which was also correlated with an increased size of cells nuclei (Köster et al. 2003). Recent studies demonstrated that Ratjadone treatment in human multiple myeloma cells rendered them more sensitive to topoisomerase Il $\alpha$ inhibitors like doxorubicin and etoposide (Turner et al. 2009, 2012). Furthermore, Ratjadone was shown to inhibit HIV infection in vitro in a concentration dependent fashion with an $\mathrm{EC}_{50}$ in the nanomolar range (Fleta-Soriano et al. 2014).

Anguinomycins are natural compounds that demonstrate an anti-tumor activity with high potency. Similar to LMB, they are isolated from Streptomyces species (Hayakawa et al. 1995). These compounds were shown to possess a selective cytotoxicity against transformed cells, which is thought to be based on the interference with the tumor suppressor Rb (Hayakawa et al. 1995). Anguinomycins block nuclear export by inhibiting CRM1 in the nanomolar range. It was shown that an Anguinomycin analog with a truncated polyketide chain can maintain an inhibitory activity at concentrations above $25 \mathrm{nM}$. This inhibitory activity of the shortened Anguinomycin led to the identification Goniothalamin (a related natural compound) as another inhibitor of nuclear export (Bonazzi et al. 2010).

Ratjadones and Anguinomycins demonstrate high structural similarity to LMB (Table 1) as these compounds have polyketide chain with a terminal lactone ring. Structural characterization of Ratjadone $A$ and Anguinomycin A bound to CRM1 showed that both compounds covalently bind CRM1 through the reactive cysteine in an identical mechanism to LMB (Sun et al. 2013). Due to their high toxicity these compounds were not tested in vivo (Turner et al. 2012). 


\section{Other natural CRM1 inhibitors}

Over the last decade several compound from plant origin were identified as nuclear export inhibitors. This includes the compounds Valtrate and Acetoxychavicol acetate isolated from Valeriana fauriei and Alpinia galangal respectively (Tamura et al. 2010; Ye and Li 2006). These compounds were shown to inhibit CRM1 mediated nuclear export of HIV-Rev protein and influenza viral RNP. Both Valtrate and Acetoxychavicol acetate bind human CRM1 covalently through Cys528 (Murakami et al. 2002; Tamura et al. 2009). Curcumin is another natural compound that demonstrated inhibitory activity of nuclear export. It is an ancient spice that is isolated from the plant Curcuma longa and widely used in traditional medicine (Aggarwal et al. 2007). CRM1 was identified as a cellular target that directly interact with Curcumin. It was shown that Curcumin treatment triggers the nuclear retention of FOXO1 and induce the upregulation of p73 and p27 expression in HeLa cells (Niu et al. 2013). Another example is piperlongumine, which is a natural alkaloid isolated from Piper longum. Piperlongumine exhibited anti-tumor activities and it was shown to covalently bind CRM1 through the Cys528 in a similar fashion to LMB (Bezerra et al. 2013; Niu et al. 2015). In general, CRM1 inhibitors from plant origin demonstrate a weak binding and display mild anti-tumor activity (Sun et al. 2016).

\section{Synthetic analog of LMB: KOS 2464}

KOS 2464 is a semi-synthetic LMB derivative (Table 1); it is the best studies compound in a series that was designed to sustain improved therapeutic windows with reduced toxicity compared to LMB. Several experiments in vitro and in vivo showed that KOS 2464 treatment demonstrate less off-target toxicity, yet it retains a comparable potency to LMB (Mutka et al. 2009). It was shown that KOS 2464 induces apoptosis in several p53 wild type cancer models, while in normal lung fibroblasts it causes cell cycle arrest but does not trigger apoptosis. In vivo experiments in mice showed that KOS 2464 can be tolerated at concentration 16-folds higher than LMB, which further confirmed its reduced toxicity (Mutka et al. 2009). Further studies showed that KOS 2464 treatment increased the sensitivity of drug-resistant high-density myeloma cells to topoisomerase inhibitor doxorubicin (Turner et al. 2012). Experimental observations on LMB derivatives provided a proof of concept that CRM1-mediated nuclear export can be inhibited with manageable toxicities in vivo (Hill et al. 2014).

\section{CBS9106}

CBS9106 (SL-801) is an orally active synthetic small molecule that exhibits a reversible inhibition of CRM1-mediated nuclear export (Sakakibara et al. 2011). CBS9106 was demonstrated to 
induced cell cycle arrest and apoptosis in vivo and in vitro models of several cancers including bladder, colon, breast, prostate, lung, pancreatic, kidney and skin cancer. CBS9106 treatment was associated with decreased levels of cellular CRM1. Further analysis revealed that CBS9106 binding to CRM1 triggers its proteasomal degradation. Oral administration of CBS9106 resulted in a significant decrease in tumor growth as well as a prolonged survival in tumor-bearing mice. This rendered CBS9106 a promising clinical candidate (Chen, Brooks, McDonald, Schwartz, Schneider, et al. 2015; Saito et al. 2014; Sakakibara et al. 2011). CBS9106 was already tested in preclinical trials and currently is being tested in a phase 1 clinical trials (Chen, Brooks, McDonald, Schwartz, Sakakibara, et al. 2015) (clinicaltrials.gov Anon n.d.). Experimental analysis by pulldown assays revealed that CBS9106 binds directly to wild type CRM1 but cannot bind to a Cys528 (Cys528Ser) mutant which confirmed its binding is mediated by the reactive cysteine residue (Sakakibara et al. 2011). Nevertheless, the exact mechanism of its reversible binding remains to be elucidated.

\section{$\mathrm{N}$-azolylacrylates analogs}

PKF050-638, an analog of N-azolylacrylate (Table 1), was developed in a study by Daelemans et al (Daelemans et al. 2002). PKF050-638 was shown to inhibit CRM1-depedent nuclear export of HIV-Rev protein (Daelemans et al. 2002). Its interaction with CRM1 was reported to be reversible and highly specific. Similar to LMB, PKF050-638 inhibitory mechanism is dependent on the blocking of the NES-binding cleft by the covalent modification of the reactive cysteine. Interestingly, a trans-enantiomer of PKF050-638 (PKF050-637) was observed to be completely inactive, which indicates that PKF050-638 exhibit strict molecular structural requirements (Daelemans et al. 2002).

\section{Selective inhibitors of nuclear export (SINES)}

Selective inhibitors of nuclear export (SINEs) are a novel class of nuclear export inhibitors that were developed based on a structure-assisted computational methodology known as consensus Induced Fit Docking (cFID) (Kalid et al. 2012). SINEs series comprise several compounds that exhibit slowly reversible binding towards CRM1 including the compounds: KPT127, KPT185, KPT205, KPT227, KPT249, KPT251, KPT330 (Selinexor) KPT335 (Verdinexor), and KPT8602 ( a second generation SINE compound) reviewed in (Sendino et al. 2018). At a structural level, SINE compounds are small molecules with an average molecular weight around $350 \mathrm{Da}$. They generally share a trifluoromethyl phenyl triazole scaffold with different Michael addition acceptor side chains that enables their covalent binding to the reactive cysteine (G. Gravina et al. 2014; Inoue 
et al. 2013; Lapalombella et al. 2012; Sendino et al. 2018; Sun et al. 2016; Zhang et al. 2013). Four of SINE compounds (KPT185, KPT251, KPT276, and KPT8602) (Table 1) were crystalized with T539C variant of ${ }^{S c} \mathrm{CRM} 1$. The crystal structures revealed that, unlike the lactone ring of $L M B$, the Michael addition acceptor side chains of SINE compounds are not hydrolyzed upon reactivity which rendered their binding reversable (J. Etchin et al. 2013; Haines et al. 2015; Hing et al. 2016; Lapalombella et al. 2012).

Several SINEs have been intensively evaluated in preclinical models of several hematological malignancies and solid tumors. The in vivo and in vitro preformed studies revealed that CRM1 inhibition by SINEs result in the nuclear retention and activation of several tumor suppressor proteins including IKB, Survivin, p53, NPMc mutant, p27, and FOXO proteins. This was correlated with cell cycle arrest induction, significant reduction of tumor growth and increased apoptosis in several cancer cells with only minor toxicity in normal cells (reviewed in (Sendino et al. 2018; Wang and Liu 2019)). Furthermore, several SINE compounds and most prominently Selinexor were observed to increase cells sensitivity towards multiple drugs used in cancer treatment such as bortezomib and carfilzomib (proteasome inhibitors) as well as doxorubicin (Conforti et al. 2015; Gandhi et al. 2018; G. L. Gravina et al. 2014; Senapedis, Baloglu, and Landesman 2014; Tan et al. 2014) . SINE compounds, in particular Selinexor and Verdinexor, have been evaluated in over 60 clinical trials in phases I and II. Early phase clinical trials revealed that although SINE compounds can be well tolerated, they don't exhibit adequate potency when used as single agent during clinical evaluation. On the other hand, SINE compounds when used in combination with existing drugs have shown a synergetic effect in several malignancies (summarized in (Sendino et al. 2018; Syed 2019; Wang and Liu 2019)). Currently Selinexor is being evaluated in mid and late phase clinical trials in combination with approved therapies like bortezomib and dexamethasone and in several cancers including multiple myeloma, liposarcoma, and in endometrial cancer (Wang and Liu 2019). Very recently, Selinexor received an accelerated approval in the USA for the treatment of adult patients with relapsed or refractory multiple myeloma when taken in a combination with dexamethasone (Syed 2019).

The approval of Selinexor for the treatment of multiple myeloma further demonstrates the importance of CRM1 as a clinical target. It furthermore indicates the significant progress in the development of CRM1 targeting anti-tumor drugs. However, despite its accelerated approval, Selinexor still exhibit dose-limiting gastrointestinal toxicities (Garzon et al. 2017; Wang and Liu 2019). In addition, Selinexor has been only approved for heavily treated patients who have received at least four prior multiple myeloma therapies and whose disease is refractory to at 
least two proteasome inhibitors, at least two immunomodulatory agents, and an antiCD38 monoclonal antibody (Syed 2019). Which altogether indicate that further developments of antitumor drugs as well as a better understanding of CRM1-inhibitors interactions are still necessarily required to obtain CRM1 targeting drugs with higher efficacy and lower toxicity.

\subsection{Aim of this work}

Over the past two decades, several natural and synthetic compounds were discovered or designed for targeting CRM1 as potential anti-tumor and anti-viral drugs. Structural characterization of CRM1-inhibitor complexes has been a fundamental platform that defined the molecular bases of CRM1 inhibition. Furthermore, computational and experimental structural methods have been used as a powerful tool for the development and optimization of CRM1 targeting compounds. The preformed biochemical and structural analysis of previously studied CRM1-targeting compounds revealed their common mechanism of action of by the covalent modification of a reactive cysteine residue that is located in the NES-binding cleft. To this date, all structures available of CRM1-inihibtor complexes retain yeast CRM1 from Saccharomyces cerevisiae that was genetically modified to incorporate the reactive cysteine (J. Etchin et al. 2013; Haines et al. 2015; Hing et al. 2016; Kalid et al. 2012; Lapalombella et al. 2012; Sun et al. 2013). Therefore, this work aim is to obtain structural information on inhibitors bound to human CRM1, the actual target for drug development. Furthermore, this study focusses on the biochemical and structural characterization of four novel nuclear export inhibitor candidates: compounds C3, C6, C10, and the compound DP2392-E10 (Table 2) (will be introduced in detail in following chapters). The compounds C3, C6 and C10 were chosen for this study due to their variable chemical composition and complexity (Table 2 ) which suggest they possess different mechanisms of action (Fetz et al. 2009). While DP2392E10 was chosen as it was predicted to bind outside the NESbinding cleft in the region around HEAT repeats 9 and 10, which may introduce a novel alternative mechanism of CRM1 inhibition (Chutiwitoonchai et al. 2017; Kakisaka, Mano, and Aida 2016).

Here we apply biochemical methods and combine integrative experimental and computational structural approaches to elucidate the mechanism of action of these compounds towards human CRM1. The outcome of this study would provide a better understanding of human CRM1 inhibition at a molecular level and would provide a new aspect on CRM1 inhibition by alternative mechanisms of action. 
Table 2: Novel nuclear export inhibitors examined in this study.

Inhibitor
(Fetz et al. 2009) $\begin{gathered}\begin{array}{c}\text { Chemical } \\ \text { Formula } \\ \text { Weight (Da) }\end{array} \\ \text { (Fetz et al. 2009) }\end{gathered}$


This manuscript was submitted to the Journal of Medicinal Chemistry and is currently under review

\title{
Chapter 2: Characterization of inhibition reveals distinctive properties for human and Saccharomyces cerevisiae CRM1
}

\author{
Alaa Shaikhqasem, Achim Dickmanns, Piotr Neumann and Ralf Ficner* \\ Department of Molecular Structural Biology, Institute of Microbiology and Genetics, GZMB, \\ Georg-August-University Göttingen, 37077 Göttingen, Germany
}

Keywords: Nuclear transport, exportin 1, cancer, drugs, inhibitor, viral diseases

\begin{abstract}
CRM1 (chromosome region maintenance 1) is a major nuclear export receptor that has been identified as nuclear export factor for many tumor suppressor proteins as well as viral RNA and ribonucleoproteins (RNPS). This renders CRM1 a particularly interesting target for therapeutic intervention in diverse cancer types and viral diseases. Structural studies of Saccharomyces cerevisiae CRM1 ( $\left.{ }^{S C} \mathrm{CRM} 1\right)$ bound to different inhibitors defined the molecular basis for CRM1 inhibition. Nevertheless, to date there is no structural information available for inhibitors bound to human CRM1 ( $\left.{ }^{H s} \mathrm{CRM} 1\right)$, the actual target for drug development. Here, we present the crystal structure of the natural nuclear export inhibitor Leptomycin B (LMB) bound to human CRM1RanGTP complex. Moreover, we demonstrate the differences in binding affinities between ${ }^{S c} \mathrm{CRM} 1$ and ${ }^{\mathrm{Hs}} \mathrm{CRM} 1$ towards the Nuclear Export Signal peptide from Protein Kinase A Inhibitor (PKI-NES) as well as several potential CRM1 inhibitors. Our data show that despite the high sequence conservation and structural similarity in the NES-binding cleft region, ${ }^{S c} \mathrm{CRM} 1$ exhibits 16-fold lower binding affinity than ${ }^{H s}$ CRM1 towards PKI-NES. Furthermore, in contrast to ${ }^{H s} \mathrm{CRM} 1$, our competition assay revealed that changing Thr539 to cysteine in ${ }^{5 c} \mathrm{CRM} 1$ did not result in binding of some of the inhibitors. Taken together, our data indicate the importance of using ${ }^{H s} \mathrm{CRM} 1$ for molecular analysis and development of novel anti-tumor and anti-viral drugs.
\end{abstract}




\subsection{Introduction}

Nucleocytoplasmic transport is a fundamental process in all eukaryotes and its essential for maintaining the intracellular distribution of various signaling molecules, transcription factors and cell cycle regulators [1]-[4]. The transport of molecules between the cytoplasm and the nucleus takes place through the nuclear pore complexes (NPCs). While small molecules can passively transverse the NPCs, the transport of molecules larger than $40 \mathrm{kDa}$ is mediated via transport receptors belonging to the importin- $\beta$ super family termed importins and exportins [5]-[7]. CRM1 (chromosome region maintenance 1 ) is the major export receptor that mediates the nuclear export of a wide range of proteins and ribonucleoprotein complexes in conjunction with a small GTPase in its GTP bound form (RanGTP) [8], [9]. Interestingly, CRM1 has been identified as nuclear export factor for many tumor suppressor proteins such as Rb, APC, p53, p21 and p27 as well as established cancer drug targets like topoisomerase II $\alpha$ and BCR-ABL [10]-[12]. In addition, elevated CRM1 expression has been observed in ovarian and cervical cancer cells and it is correlated with poor overall survival in several cancer types [13]. Furthermore, CRM1 mediates the nuclear export of a wide range of viral RNA and ribonucleoproteins (VRNP) and therefore has a key role in multiple viral diseases including influenza, HIV-1, and human T-cell leukemia virus type-1 [14]-[16]. This renders CRM1 a particularly interesting target for therapeutic intervention in diverse cancer types and viral diseases [17], [18].

On a structural level CRM1 consists of 21 HEAT repeats and thus retains a common structural feature of transport receptors belonging to the importin- $\beta$ super family [8]. CRM1 mechanism of transport relies on the recognition of the cargo proteins through their leucin-rich nuclear export signals (NESs), which are consensus sequences that comprise 4-5 key hydrophobic residues (Ф0Ф4) with characteristic spacings. The NES binds CRM1 in a hydrophobic cleft located at the outer convex surface of CRM1 between HEAT repeats 11 and 12 (NES-binding cleft). The cleft contains 5 hydrophobic pockets responsible for the specific binding of the hydrophobic side chains of the NES key residues [19]-[22]. Leptomycin B (LMB), a classical and well-studied CRM1 inhibitor, interferes with the NES binding by blocking the NES-binding cleft through an irreversible covalent conjugation with the reactive cysteine residue Cys528 in human CRM1 $\left({ }^{H s} \mathrm{CRM} 1\right)$ (corresponds to Cys529 in Schizosaccharomyces pombe CRM1) [23]-[25]. Clinical tests revealed a dose-limiting toxicity of LMB and severe side effects [26]. This induced a continuous search and development of alternative compounds lacking those side effects with the focus of finding CRM1 inhibitors which could be used as anti-tumor and anti-viral drug candidates [27]-[34]. A prominent example is the development of a class of CRM1 inhibitors referred to as selective inhibitors of nuclear 
export (SINEs), which were designed to bind CRM1 in the NES-binding cleft through the reactive cysteine [35], [36]. Unlike LMB the SINE compounds exhibited slowly reversible covalent binding, which rendered them significantly less toxic and more promising than LMB [37]. Recently, the SINE compound KPT330 (Selinexor) was approved in the USA for the treatment of adult patients with relapsed or refractory multiple myeloma when taken in a combination with dexamethasone [38]. This pronounced progress in the development of CRM1 inhibitors further emphasizes its significance as a clinical target.

Structural analysis of several inhibitors bound to CRM1 defined the molecular basis of CRM1 inhibition and it became an essential platform for designing and optimizing potential CRM1targeting drugs [36], [39]-[43][44]. These crystallographic studies on CRM1-inhibitor complexes were carried out using Saccharomyces cerevisiae CRM1 ( $\left.{ }^{S c} \mathrm{CRM} 1\right)$ in a complex with Ran and RanBP1, despite its $47 \%$ sequence identity with ${ }^{H s} \mathrm{CRM} 1$ [45]. Utilization of ${ }^{S c} \mathrm{CRM} 1$ for structural analysis required an exchange of Thr539 to cysteine, which is the corresponding residue Cys528 in ${ }^{H s}$ CRM1 known to be necessary for binding of the studied inhibitors [36], [39]-[42]. Consequently, there is no structural information available for inhibitors bound to ${ }^{H s} \mathrm{CRM} 1$ to date. In order to fill this gap data and to structurally explain differences in inhibitors binding between ${ }^{H s} \mathrm{CRM} 1$ and ${ }^{S c} \mathrm{CRM} 1$, we have determined the crystal structure of LMB bound to ${ }^{H s} \mathrm{CRM} 1-\operatorname{RanGTP}$ complex. We have also performed a biochemical characterization of potential CRM1 inhibitors: compounds C3, C6 and C10 [46] as well as the compound DP2392-E10 [47]. Using fluorescence polarization (FP) binding/competition assay we could show differences in binding affinities of NES from protein kinase $\mathrm{A}$ inhibitor (PKI) as well as the compounds $\mathrm{C} 3$ and $\mathrm{C} 6$ when bound to ${ }^{\mathrm{Hs}} \mathrm{CRM} 1$ and ${ }^{S c} \mathrm{CRM} 1$. Our data show that despite the high structural similarity of ${ }^{\mathrm{Hs}} \mathrm{CRM} 1$ and ${ }^{\mathrm{Sc} C R M} 1 \mathrm{NES}-$ binding clefts, they exhibit different binding properties towards PKI-NES and the compounds C3 and C6.

Our study demonstrates the importance of using ${ }^{H s} \mathrm{CRM} 1$ for structural characterization of drug candidates as well as the accurate determination of their binding potency.

\subsection{Materials and Methods}

\subsubsection{Plasmid construction}

The sequence encoding the full-length human CRM1 $1^{1-1071}$ was codon optimized and synthesized for expression in Escherichia coli (E. coli) using the GeneArt platform (ThermoFisher). The codon optimized sequence was cloned into pET21a vector (Novagen) as an N-terminal $\mathrm{T}_{7}$-tagged and C- 
terminal His6-tagged fusion protein using BamHI and Xhol restriction sites. Truncated versions of CRM1 with the C-terminal Helix ( $\triangle 1037-1071)$, the acidic loop mutations ( ${ }^{430} \mathrm{VLV} 432$ to $\left.A A A\right)$, and C528S mutant were obtained via site directed mutagenesis and verified by sequencing. The CRM1 gene from Saccharomyces cerevisiae was amplified by polymerase chain reaction from a total DNA preparation. The amplified DNA product was cloned into PET21a vector (Novagen) using the restriction sites BamHI and Xhol. T539C mutation was introduced via site directed mutagenesis on the wild type plasmid and confirmed by sequencing.

\subsubsection{Protein expression and purification}

Wild type and CRM1 mutants from human and yeast were expressed in E. coli BL21- (DE3) cells in 2 YT medium supplemented with ampicillin. Expression cultures were incubated at $37^{\circ} \mathrm{C}$ until the optical density $\left(\mathrm{OD}_{600}\right)$ reached 0.3 . The cultures were then further incubated at $18^{\circ} \mathrm{C}$. Protein expression was induced by adding Isopropyl $\beta$-D1-thiogalactopyranoside (IPTG) to $0.1 \mathrm{mM}$ when the $\mathrm{OD}_{600}$ reached 0.6 . After 18 hours the cells were harvested, and the pellets were frozen in liquid nitrogen and stored at $-80^{\circ} \mathrm{C}$ until further use. For lysis, cells were resuspended in HisTrap loading buffer (50 mM HEPES pH 7.5, 500 mM NaCl, 30 mM imidazole, 2 mM MgCl , 10\% glycerol, $4 \mathrm{mM}$ 2-mercaptoethanol) supplemented with protease inhibitor cOmplete ULTRA Tablets (1 tablet / $50 \mathrm{ml}$ ) (Roche). Cells were lysed by running the suspension for 7 cycles in a microfluidizer (Microfluidics). The resulting lysate was cleared by centrifugation for $30 \mathrm{~min}$ at $50,000 \times \mathrm{xg}$ at $4{ }^{\circ} \mathrm{C}$. $\mathrm{Ni}^{2+}$-chelate affinity chromatography was performed by applying the cleared lysate to a HisTrap FF column (GE Healthcare) pre-equilibrated in HisTrap loading buffer. Immobilized protein was eluted in HisTrap loading buffer containing 400 mM imidazole. Remaining impurities were removed via anion exchange chromatography after desalting the sample by dialysis in dialysis buffer (50 mM HEPES pH 7.5, 50 mM NaCl, 2 mM MgCl , 5\% glycerol, 4 mM 2-mercaptoethanol). The sample was loaded on a Source 300 anion exchange chromatography column (GE Healthcare) pre-equilibrated in Source 30Q low salt buffer (50 mM HEPES pH 7.8, 50 mM NaCl, 2 $\mathrm{mM} \mathrm{MgCl}_{2}, 6 \mathrm{mM}$ 2-mercaptoethanol). Elution was performed in 0-70\% gradient of Source $30 \mathrm{Q}$ high salt buffer (50 mM HEPES pH 7.8, 400 mM NaCl, 2 mM MgCl 2,6 mM 2-mercaptoethanol) over 30 column volumes. Fractions containing CRM1 were pooled, concentrated using 50000 MWCO centrifugal concentrator (Merck Millipore) and used for crystallization and biochemical assays.

For pull down assay full length human CRM1_MBP fusion protein was expressed from the plasmid pQE60 as C-terminal His 6 -tagged. The MBP fusion protein was recombinantly expressed in E. coli 
SG13009 cells grown in 2YT medium supplemented with ampicillin and kanamycin. Protein expression was induced by adding $0.1 \mathrm{mM}$ IPTG when the $\mathrm{OD}_{600}$ reached 1.2 at $18{ }^{\circ} \mathrm{C}$. Cells were harvested 18 hours after induction and the pellets were frozen in liquid nitrogen and stored at $80{ }^{\circ} \mathrm{C}$. CRM1_MBP was purified by $\mathrm{Ni}^{2+}$-chelate affinity chromatography and anion exchange chromatography using the same protocol as described above except for the buffers content of 12 mM 2-mercaptoethanol. On top of that CRM1_MBP-containing eluate from Source 300 column was further purified by gel filtration on a Superdex 200 column (GE Healthcare) preequilibrated in gel filtration buffer (50 mM HEPES pH 7.5, $50 \mathrm{mM} \mathrm{NaCl}, 2 \mathrm{mM} \mathrm{MgCl}$, $12 \mathrm{mM}$ 2mercaptoethanol, $10 \%$ Glycerol).

Human SPN1 1-360 was expressed as previously described [48] and purified as described in [49]. Expression and purification of human RanGTP1-180, Q69L [49] was performed as described in [50].

\subsubsection{Preparation and crystallization of ${ }^{H s} \mathrm{CRM} 1-{ }^{H s}$ RanGTP-LMB complex}

For crystallization purpose a HEAT 9 loop mutant ( ${ }^{430} \mathrm{VLV}^{432}$ to AAA) of the C-terminally truncated ( $\alpha$-helix; $\Delta 1037-1071){ }^{H s} \mathrm{CRM} 1$ was used. The complex was prepared by mixing ${ }^{H s} \mathrm{CRM} 1{ }^{\Delta 1037-1071}$, $430 V L V 432$ to AAA with ${ }^{H s}$ RanGTP ${ }^{1-180, ~ Q 69 L}$ at a 1:1 molar ratio, LMB was then added to the protein mixture at a 1:2 molar ratio and the mixture was incubated on ice for an hour. Subsequently, the mixture was dialyzed at $4{ }^{\circ} \mathrm{C}$ in a buffer containing $50 \mathrm{mM} \mathrm{HEPES} \mathrm{pH} \mathrm{7.8,} 50 \mathrm{mM} \mathrm{NaCl}, 2 \mathrm{mM}$ $\mathrm{MgCl}_{2}$, and $6 \mathrm{mM}$ 2- mercaptoethanol. After dialysis LMB was re-supplemented in a 1:1 molar ratio and the sample was further incubated for 1 hour on ice. Before crystallization the sample was cleared by centrifugation for $10 \mathrm{~min}$ at $20,000 \mathrm{xg}$ and at $4{ }^{\circ} \mathrm{C}$ to remove any formed precipitate.

Crystallization trials of the prepared complex were set up using a wide range of sparse matrix screens. The screening was carried out using sitting drop vapor diffusion in 96-well 3-drop MRC SWISSCI crystallization plates (Molecular Dimensions). The trials yielded crystal clusters or micro crystals in multiple conditions. Nonetheless, reasonable size single crystals $(110 \times 90 \times 140$ microns) grew within 3 days at $4^{\circ} \mathrm{C}$ in the condition Morpheus $\mathrm{H} 10$ [51] (10\% w/v polyethylene glycol 8000, 0.1 M M-buffer pH 8.5, 20\% w/v ethylene glycol, 0.02 M M-Amino Acids) mixed in equal volume with the complex concentrated to $3 \mathrm{mg} / \mathrm{ml}$. Crystals were cryo-protected by soaking them in a reservoir solution supplemented with $5 \%$ ethylene glycol, 5\% PEG8000, 10\% glycerol.

Diffraction images were collected at PETRA III EMBL beamline P14 (DESY, Hamburg, Germany) and processed with the XDS package [52]. Data collection and processing statistics are 
summarized in Table 1. An orthorhombic lattice with unit cell parameters of $a=122.29 \AA, b=$ $151.33 \AA, c=234.84 \AA$ was determined for the crystals containing ${ }^{H s}$ CRM1-Hs $R$ anGTP-LMB complex. The cell content analysis indicated the presence of one molecule in the asymmetric unit $\left(V_{m}=3.7 \AA^{3} / D a\right.$, corresponding solvent content of $\left.66.7 \%\right)$.

Table 1: Crystallographic data collection and refinement statistics.

\begin{tabular}{|c|c|}
\hline & ${ }^{H s}$ CRM1- ${ }^{H s} \operatorname{RanGTP-LMB}$ \\
\hline \multicolumn{2}{|l|}{ Crystallographic data } \\
\hline Beamline & Petra III-P14 \\
\hline Wavelength (Å) & 0.9763 \\
\hline Resolution range $(\AA)^{*}$ & $127.20-3.20(3.39-3.20)$ \\
\hline Unique reflections & 36131 \\
\hline Redundancy & $4.8(4.9)$ \\
\hline Completeness (\%) & $99.5(98.8)$ \\
\hline Space group & 1222 \\
\hline$a, b, c(\AA)$ & $122.29,151.33,234.84$ \\
\hline$\alpha, \beta, \gamma\left({ }^{\circ}\right)$ & $90,90,90$ \\
\hline $\mathbf{R}_{\text {meas }(\%)}$ & $6.5(101.4)$ \\
\hline$I / \sigma(I)$ & $17.63(1.96)$ \\
\hline $\mathrm{CC}_{1 / 2}$ & $99.9(74.9)$ \\
\hline \multicolumn{2}{|l|}{ Refinement statistics } \\
\hline $\mathbf{R}_{\text {work }} / \mathbf{R}_{\text {free }}$ & $0.21 / 0.24$ \\
\hline No. of atoms & 9678 \\
\hline Average $B$ factor $\left(\AA^{2}\right)$ & 114 \\
\hline \multicolumn{2}{|l|}{ Root mean square deviation } \\
\hline Bonds (Å) & 0.006 \\
\hline Angles (degree) & 0.24 \\
\hline \multicolumn{2}{|l|}{ Ramachandran plot } \\
\hline Favoured (\%) & 97.29 \\
\hline Allowed (\%) & 2.63 \\
\hline Outlier (\%) & 0.08 \\
\hline \multicolumn{2}{|l|}{ PDB code } \\
\hline & 6TVO \\
\hline
\end{tabular}

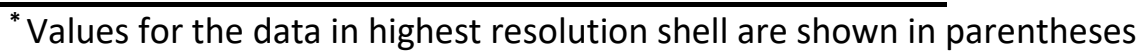

\subsubsection{Structure determination and refinement}

The structure was solved by means of molecular replacement using PHASER [53] with the crystal structures of the CRM1-RanGTP complex (PDB-ID 3NC1) [22] as the search model. LMB was modeled manually in COOT [54]. The structure was improved by iterative cycles of refinement and manual rebuilding in PHENIX [55] and COOT respectively. The presence of LMB was verified using a Polder omit map [56]. Ramachandran statistics were calculated in COOT. Analysis of the protein-ligand interactions including hydrophobic and hydrogen bonding interactions was 
performed by LigPlot+ [57]. Figures were generated with PyMol (The PyMOL Molecular Graphics System, Version 1.8 Schrödinger, LLC).

\subsubsection{Pull-down assay}

$0.5 \mathrm{nmol}$ CRM1_MBP fusion protein were immobilized on $50 \mu \mathrm{L}$ amylose resin (NEB) equilibrated in pull-down buffer (20 mM Tris pH 7.5, $150 \mathrm{mM} \mathrm{NaCl}, 2 \mathrm{mM} \mathrm{MgCl}$, and $1 \mathrm{mM} \mathrm{DTT}$ ). The beads were incubated with proteins of interest ( $4 \mathrm{nmol}$ each) and inhibitors of interest (100 nmol each except for LMB $20 \mathrm{nmol}$ ) in a total volume of $150 \mu \mathrm{L}$ for $20 \mathrm{~min}$ at $22{ }^{\circ} \mathrm{C}$. Afterwards the beads were washed three times with $1 \mathrm{ml}$ pull-down buffer each. Bound proteins were eluted in $20 \mu \mathrm{L}$ pull down buffer supplemented with $15 \mathrm{mM}$ maltose. After elution samples were centrifuged for $10 \mathrm{~min}$ at $20,000 \times \mathrm{g}$ and at $4^{\circ} \mathrm{C}$. Supernatant was then carefully transferred into clean tube, mixed with 2X SDS-PAGE loading dye, and analyzed by SDS-PAGE followed by Coomassie staining. The intensity of CRM1 and SPN1 bands were quantified by ImageJ software [58].

\subsubsection{Fluorescence polarization binding assay}

Fluorescence polarization binding assays were applied to measure the binding affinity of CRM1 wild type and mutants to PKI $\Phi^{0}$ Leu NES (LNELALKLAGLDIK) labeled with carboxyfluorescein via its C-terminal lysine. PKI $\Phi^{0}$ Leu NES is a modified PKI-NES with an additional leucine in the Ф0 $^{2}$ position which results in higher binding affinity to CRM1 compared to the classical PKI NES that contains four critical hydrophobic residues (Ф1-Ф4) [22]. Increasing concentrations of CRM1 were mixed with $40 \mathrm{nM}$ NES in the absence or presence of ${ }^{H s}$ RanGTP ${ }^{1-180,}$ Q69L in molar excess to CRM1. The assay was followed in a buffer containing $20 \mathrm{mM}$ Tris- $\mathrm{HCl}, \mathrm{pH} \mathrm{7.5,} 130 \mathrm{mM} \mathrm{NaCl}, 2$ $\mathrm{mM} \mathrm{MgCl}_{2}, 1 \mathrm{mM} \mathrm{DTT}$, and 0.005\% (w/v) digitonin (Sigma-Aldrich). All reactions were performed in $150 \mu \mathrm{L}$ and measured as a set of triplicates. For all measurements an excitation wavelength of $480 \mathrm{~nm}$ was set, and the emission was detected at $530 \mathrm{~nm}$ for $500 \mathrm{~ms}$. Measurements were performed using VICTOR Nivo Multimode Microplate Reader (PerkinElmer). Dissociation constants $(\mathrm{Kd})$ were determined by nonlinear regression using the analysis software OriginPro $9.1[59]$.

\subsubsection{Fluorescence polarization competition assay}

The potency of inhibitors binding to CRM1 wild type and mutants was evaluated using fluorescence polarization competition assay. Inhibitors - prepared in a dilution series or added to a specific concentration - were mixed with $40 \mathrm{nM}$ fluorescently labeled PKI $\Phi^{0}$ Leu NES and 100 nM CRM1 in a total volume of $150 \mu \mathrm{L}$ under the same buffer conditions used for fluorescence 
polarization binding assay. For inhibitor 10 , as it exhibited autofluorescence, FP measurements of the inhibitor alone were performed in the same buffer conditions and were subtracted from the binding measurements. Measurements and data evaluation were performed under the same conditions for the fluorescence polarization binding assay.

\subsubsection{PKI $\Phi^{0}$ Leu NES and CRM1 inhibitors}

Fluorescently labeled PKI $\Phi^{0}$ Leu NES was synthesized by EMC microcollections (Germany). DP2392-E10 was synthesized by Ambinter (France). Leptomycin B was supplied by Iris-Biotech (Germany). Compound C3 was supplied by ChemDiv (USA). Compound C6 (synthesized by ChemBridge Corporation, USA) and compound C10 (synthesized by Chemical Block, Russia) were ordered via MolPort.

\subsection{Results and Discussion}

\subsubsection{Structure determination of human CRM1-LMB complex}

To get an insight into inhibitor bound- ${ }^{H s}$ CRM1 structure, LMB was chosen as the representative compound due to its high binding affinity and stability in aqueous solutions. However, in spite of extensive screening, crystallization of the ${ }^{H s}$ CRM1-LMB complex in the absence or presence of RanGTP failed to yield crystals, most likely due to conformational flexibility of wild type ${ }^{H s}$ CRM1 [60]. Previous studies have shown that CRM1 samples in solution between two extreme conformations: a superhelical extended and pitched conformation as well as a compact toroidlike conformation [8], [61]. The compact conformation is characterized by the opening of the NES-binding cleft and therefore displays enhanced NES/inhibitor binding affinity. The observed conformational transition of CRM1 as well as the state of the NES-binding cleft are mainly regulated by two structural elements: the C-terminal Helix and the HEAT9 loop (acidic loop). The extended conformation is stabilized by the $\mathrm{C}$-terminal helix bridging the central opening of CRM1 and interacting with HEAT repeats forming the NES-binding cleft. Moreover, in this CRM1 conformation the acidic loop (specifically residues Val441, Leu442, and Val443 of ${ }^{5 c} \mathrm{CRM} 1$ which correspond to Val430, Leu431, and Val432 of "HsCRM1) is found in the so-called "flipped back" position causing it to pack against the back of the NES-binding cleft which adopts a closed conformation that is not accessible by NES or inhibitors [60]-[62]. In order to shift the ${ }^{H s}$ CRM1 conformational equilibrium towards the compact conformation facilitating binding of LMB, we truncated the C-terminal helix ( $\Delta 1037-1071)$ and changed the hydrophobic residues ${ }^{430} \mathrm{VLV}^{432}$ in the acidic loop to alanine (AAA). A combination of these mutations has been described to 
significantly increase Mus musculus CRM1 (MmCRM1) affinity to PKI-NES, indicating for a significant shift towards the compact conformation [63]. Using FP binding assays, we could observe that synergistic effect of both mutations resulted in an increase of ${ }^{\mathrm{Hs}} \mathrm{CRM} 1$ affinity to PKI $\Phi^{0}$ Leu NES in the absence of RanGTP (supplementary figure 1). This confirms a similar effect of the acidic loop and the C-terminal helix on NES binding in ${ }^{H s} \mathrm{CRM} 1$ as in ${ }^{\mathrm{Mm}} \mathrm{CRM} 1$. Consequently, ${ }^{H s} \mathrm{CRM} 1{ }^{\Delta 1037-1071,430 \mathrm{VLV} 432}\left({ }^{H s} \mathrm{CRM}^{\Delta}\right)$ was used to set up crystallization screens which succeeded to yield reasonably diffracting single crystals of ${ }^{H s} \mathrm{CRM} 1-{ }^{H s}$ RanGTP-LMB complex. Although multiple mutations were introduced to CRM1, in the presence of RanGTP the ${ }^{H s} \mathrm{CRM} 1^{\Delta}$ mutant and the wild type CRM1 demonstrate the same binding affinity $(\mathrm{Kd}=35-37 \mathrm{nM})$ towards PKI-NES (supplementary figure 1). This indicates that the obtained complex structure containing RanGTP is a representative model for the wild type ${ }^{H s}$ CRM1.

The complex ${ }^{H s} \mathrm{CRM}_{1}{ }^{\Delta}{ }^{H s}$ RanGTP-LMB crystalized in the space group 1222 . The phase problem was solved by means of Molecular Replacement using the ${ }^{M m} \mathrm{CRM1}{ }^{H s}$ RanGTP complex structure (PDB code $3 \mathrm{NC1}$ ) as a search model. The resulting crystal structure of ${ }^{H s} \mathrm{CRM} 1-{ }^{H s}$ RanGTP-LMB was refined at $3.2 \AA$ resolution (Table 1). The complex structure reveals CRM1 adapting the ring-like compact conformation (supplementary figure $2 \mathrm{~A}$ ), which is stabilized by the numerous interactions between the $\mathrm{N}$ - and $\mathrm{C}$ - terminal regions. The acidic loop, detached from the back of the NES-binding cleft, adopts "seatbelt"-like conformation that secures RanGTP interaction with CRM1. The NES-binding cleft located in the hydrophobic cleft between HEAT repeats $11 \mathrm{~A}$ and $12 \mathrm{~A}$ is in an open state that exhibits similar dimensions to the cleft occupied with Snurportin1 (SPN1) NES (CRM1-SPN1-RanGTP complex, PDB ID: 3GJX). An excess electron density located in the cleft clearly resembles the LMB molecule (supplementary figure $2 \mathrm{~B}$ ), a polyketide that consists of an unsaturated, branched fatty acid chain with a terminal $\alpha, \beta$-unsaturated d-lactone ring (Figure $1 \mathrm{~A}$ ). LMB binds to Cys528 via Michael addition reaction, which results in its irreversible covalent conjunction of the reactive cysteine and the hydrolysis of its terminal lactone ring [42]. While the hydrolyzed lactone ring is anchored by Cys528, the rest of the polyketide chain extend towards the lower part of the NES-binding cleft blocking approximately $70 \%$ of its accessible surface (supplementary figure 2B, Figure 1B). A more detailed analysis of LMB interactions shows that the hydrolyzed (open) lactone ring is stabilized via polar interactions by surrounding positively charged residues including hydrogen bonding with Lys537 and Lys568 (Figures $1 \mathrm{~B}$ and $1 \mathrm{C}$ ). The terminal carboxyl group at the other end of the polyketide chain is favored by electrostatic interactions with the amino group of Lys514. Except for the $\beta$-hydroxyketone group, the rest of the polyketide chain binds to ${ }^{H s} \mathrm{CRM} 1$ via hydrophobic interactions with 
the surrounding residues Ile521, Lys522, Leu525, and Glu529 of HEAT repeat 11A and residues His558, Phe561, Thr564, and Phe572 of HEAT repeat 12A. Furthermore, LMB reaches deeper into the NES-binding cleft making hydrophobic contacts with Met545 and Ala541 of HEAT repeat 11B.

The stabilization of the hydrolyzed $\alpha, \beta$-unsaturated lactone in addition to the tight hydrophobic interaction along the NES-binding cleft results in a persistent irreversible blockage of CRM1 mediated nuclear export, which could explain its significant toxicity during its clinical trials [26].

A

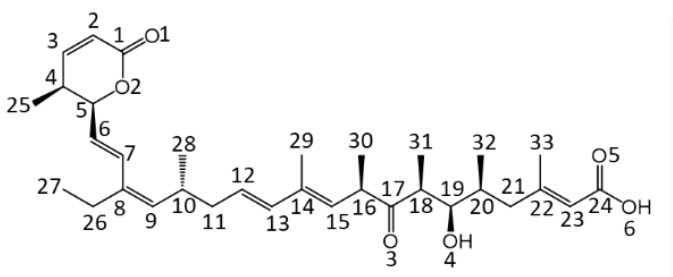

B

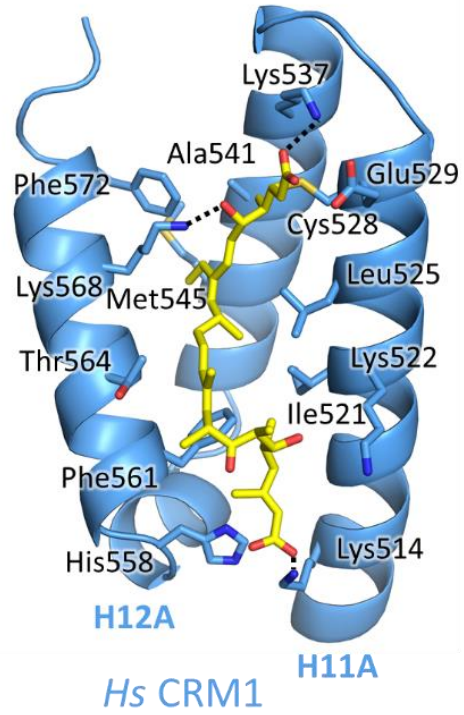

C
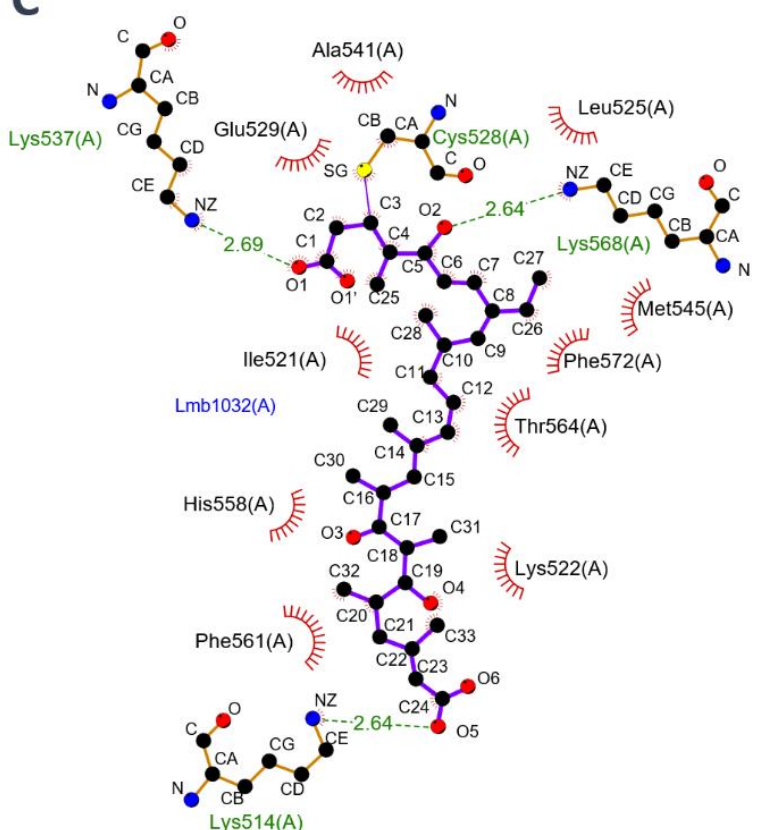

Ligand bond
Non-ligand bond
Hydrogen bond

His 53 Non-ligand residues involved in hydrophobic contact(s)

Corresponding atoms involved in hydrophobic contact(s)

Figure 1: LMB binding in the NES-binding cleft of human CRM1. (A) A chemical drawing of LMB. (B) A detailed view of the NES-binding cleft (cartoon representation) showing HEAT repeat helices $11 \mathrm{~A}$ and $12 \mathrm{~A}$ as well as the amino acids (depicted as sticks) that are involved in LMB binding. The bound LMB is shown as a stick model (carbon in yellow, oxygen in red and nitrogen in blue). Hydrogen bonds are shown as black dashed lines. (C) Schematic representation of interactions between LMB and the NES-binding cleft generated using LigPlot+ [57]. Interatomic distances of presented hydrogen bonds are given in $\AA$. 


\subsubsection{Comparison of LMB binding in human CRM1 and Saccharomyces cerevisiae}

\section{CRM1}

Previous structural analysis of LMB-CRM1 interactions was performed using ${ }^{5 C} C R M 1$. The yeast CRM1 orthologue has been chosen not only because of its high structural similarity to ${ }^{H s}$ CRM1 and high sequence similarity in the NES-binding cleft region, but also due to known crystallization conditions [42]. Comparison of LMB binding in ${ }^{H s} \mathrm{CRM} 1$ and ${ }^{S c} \mathrm{CRM} 1$ unveils a very similar mode of ligand-receptor interaction mediated mainly via 14 amino acids (Figures $2 \mathrm{~A}$ and 2B). Superposition of both CRM1 structures reveals that 12 of these 14 residues exhibit a similar or almost identical conformation and mode of interaction with the bound ligand (Figure 2C).

A

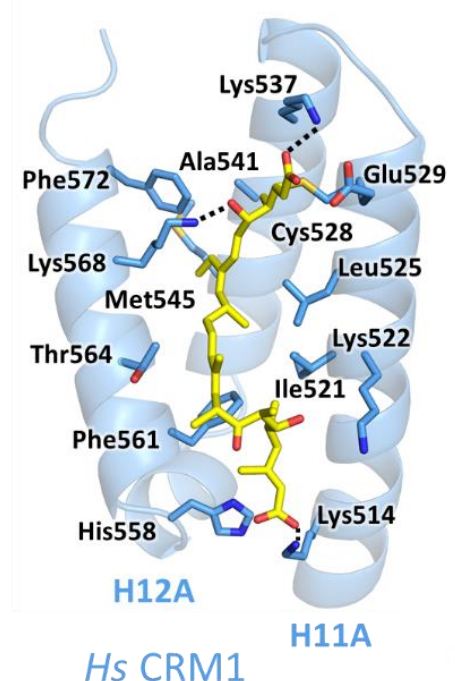

B

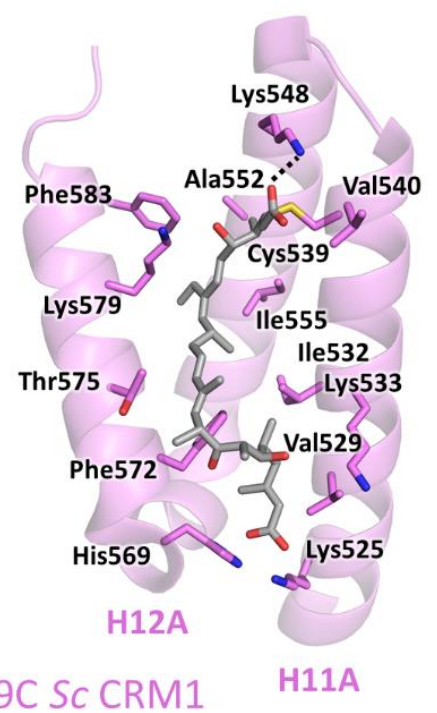

C

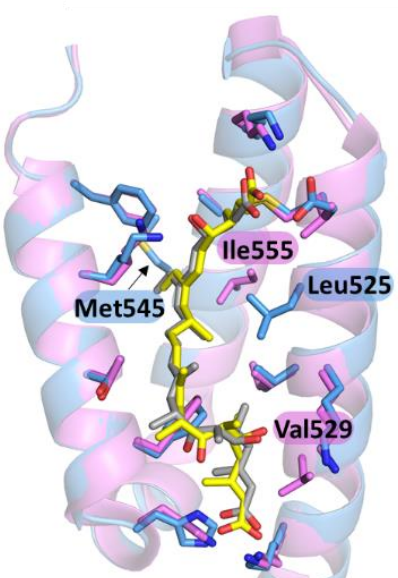

H12A

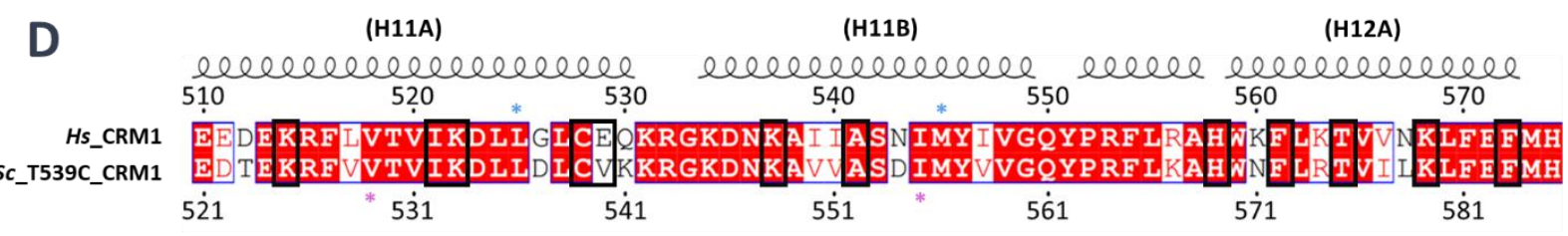

Figure 2: Comparison of LMB binding to ${ }^{H s} \mathrm{CRM} 1$ and ${ }^{S c} \mathrm{CRM} 1$. LMB interactions with the ${ }^{H s} \mathrm{CRM} 1$ NES-binding cleft are shown in (A) and with the ${ }^{S c}$ CRM1 NES-binding cleft (PDB ID: 4HAT) are shown in (B). Binding interactions were examined using LigPlot+. LMB and interacting residues are shown as sticks. Hydrogen bonds are presented as black dashed lines. (C) Superposition of ${ }^{H s}$ CRM1-LMB and ${ }^{S c} \mathrm{CRM} 1-\mathrm{LMB}$ complexes. Distinctive interacting residues are shaded (light marine in ${ }^{\mathrm{Hs}} \mathrm{CRM} 1$ and violet in ${ }^{S c} \mathrm{CRM} 1$ ) and marked with asterisks in (D). (D) Sequence alignment of wild type ${ }^{H s} \mathrm{CRM} 1$ and

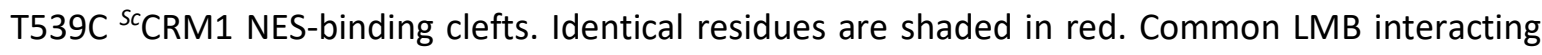
residues are highlighted in black boxes. 
In spite of these similarities, the conformation of $L M B$ observed in both proteins is not identical, as in ${ }^{H s} \mathrm{CRM} 1$ the ligand adopts more extended (by $1.5 \AA$ ) conformation than in ${ }^{\mathrm{Sc} C R M 1 .}$ In addition, the $\beta$-hydroxy-ketone moiety exhibits a more twisted conformation when compared to ${ }^{S c} C R M 1-b o u n d ~ L M B ~(F i g u r e ~ 2 C)$. The observed differences in the LMB conformation are associated with slight alterations of the LMB interaction profile in both structures. ${ }^{H s} \mathrm{CRM1-bound}$ LMB shows two distinct hydrophobic interactions with residues Leu525 and Met545, whereas ${ }^{S c}$ CRM1-bound LMB shows hydrophobic interactions with the residues Val529 and lle555 (Figure 2C). Sequence alignment of both receptors reveals that these 4 residues are strictly conserved (Figure 2D).Recently, the effect of sequence differences of the residues near the reactive cysteine has been investigated by mutating the segment ${ }^{537}{ }^{2}{ }^{2 T V K}{ }^{541}$ of ${ }^{S C} C R M 1$ to GLCEQ to mimic the ${ }^{H s} \mathrm{CRM} 1$ sequence. The structural analysis revealed that the binding mode of LMB in this modified receptor was virtually identical to ${ }^{5 c} \mathrm{CRM} 1{ }^{T 539 C}$ [42]. This suggests that the observed conformational differences of bound LMB between human and yeast CRM1 are most likely caused by the lack of sequence conservation either in the remaining part of NES-binding cleft and/or surrounding HEAT repeats. Hence, residues which are not involved in direct interactions with LMB (Figure 2D) could through allosteric effects alter the biochemical properties of the NESbinding cleft and in that way influence LMB conformation.

\subsubsection{Human and Saccharomyces cerevisiae CRM1-inhibitor interaction patterns reveal} high structural and sequence similarities

Besides LMB, several inhibitors have been structurally characterized in the recent years using the

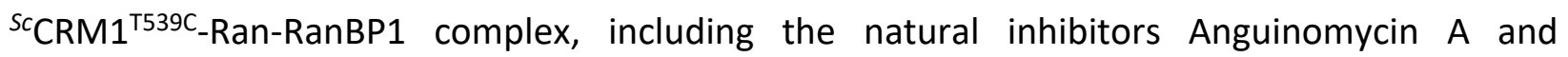
Ratjadone A as well as the synthetic SINE inhibitors KPT185, KPT251, KPT276 and KPT8602. Anguinomycin $A$ and Ratjadone A share $\alpha, \beta$-unsaturated lactone ring that bind to CRM1 via covalent conjunction to Cys539 variant of ${ }^{S c} \mathrm{CRM} 1$ (Cys528 in ${ }^{\mathrm{Hs}} \mathrm{CRM} 1$ ) in a similar mechanism to LMB [42]. On the other hand, KPT compounds share a trifluoromethyl phenyl triazole scaffold with different Michael addition acceptor side chains that allows their covalent conjugation to the reactive cysteine in the NES-binding cleft [36], [39]-[41]. Unlike the LMB-lactone ring the natural compounds, the Michael addition acceptor group of SINE-KPT compounds were shown not to hydrolyze upon binding to CRM1, which renders these compounds as better candidates for antitumor drug development [37], [64]. In order to get an insight into potential interactions of these inhibitors with ${ }^{H s} \mathrm{CRM} 1$ we performed a superposition of these complex structures based on NES clefts using ${ }^{H s} \mathrm{CRM} 1$ as reference (Figures $3 \mathrm{~A}-\mathrm{C}$ ). 


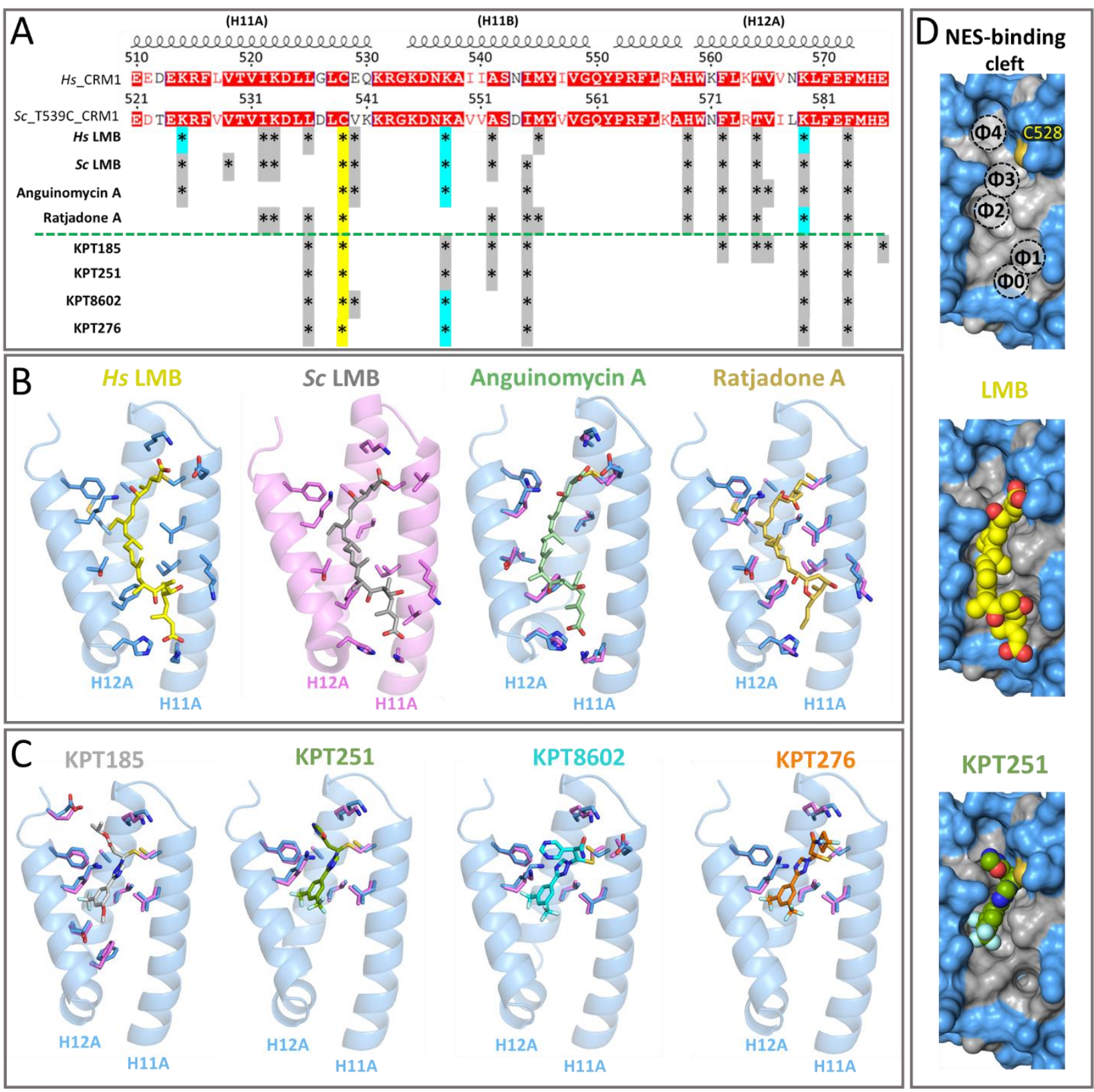

Figure 3: Structural and sequence comparison of CRM1-inhibitor interactions in human and yeast CRM1. Only the helices involved in NES binding are depicted. (A) An alignment of residues interacting with inhibitor against sequence alignment of wild type ${ }^{H s} \mathrm{CRM} 1$ and T539C ${ }^{\mathrm{Sc} C R M} 1 \mathrm{NES}-$ binding clefs. Inhibitor-CRM1 interactions were analyzed using LigPlot+. Interacting residues with each compound were annotated with an asterisk along the sequence alignment of ${ }^{S C} \mathrm{CRM} 1{ }^{\mathrm{TS} 39 C}$ and were highlighted according to the mode of interaction (covalent: yellow, hydrogen bonding: cyan, hydrophobic: grey). (B) Natural inhibitors binding to CRM1. Detailed view on LMB binding to ${ }^{H s} \mathrm{CRM} 1$ (Hs LMB) and ${ }^{S c}$ CRM1 (Sc LMB) (PDB ID 4HAT). Superpositions of Anguinomycin (PDB ID 4HAV) A and Ratjadone A (PDB ID 4HAU) in LMB bound ${ }^{H s}$ CRM1 NES-binding cleft with LMB removed. (C) Superposition of synthetic KPT-SINE compounds KPT185 (PDB ID 4GMX), KPT251 (PDB ID 4GPT), 
KPT8602 (PDB ID 5JU), and KPT276 (PDB ID 4WVF) in LMB bound ${ }^{H s}$ CRM1 NES-binding cleft with $\mathrm{LMB}$ removed. Inhibitors, their interacting residues, and the corresponding residues in human NESbinding cleft are shown as sticks (light marine in ${ }^{H s} C R M 1$ and violet in ${ }^{S C} C R M 1$ ) in (B) and (C). (D). Blocking of the NES-binding cleft by natural and synthetic KPT-SINE compounds. Surface representation of LMB bound human CRM1 NES-binding cleft. (upper) LMB was removed to show the respective $\Phi$ pockets (Ф0- $\Phi 4)$ in which NESs are recognized. (middle) LMB is depicted in a sphere mode. (lower) KPT251 superpositioned in the in LMB bound ${ }^{H s}$ CRM1 NES-binding cleft with LMB removed. Cys528 is colored yellow.

Comparison of the NES-binding cleft of ${ }^{H s} \mathrm{CRM} 1$ with these of ${ }^{\mathrm{Sc}} \mathrm{CRM} 1$ complexes unveiled identical or similar positioning of the amino acids directly involved in binding of inhibitor molecules. Conserved in sequence (Figure 3A), residues interacting with Anguinomycin $A$ and Ratjadone A (Figure $3 \mathrm{~B}$ ) as well as these interacting with SINE-KPT compounds (Figure 3C) exhibited high similarity or were identical in terms of side chain position and conformation with respect to their corresponding residues in ${ }^{H s} \mathrm{CRM} 1$. The only exception with respect to sequence conservation is Val540 of ${ }^{S c}$ CRM1 (equivalent to Glu529 in ${ }^{H s} \mathrm{CRM} 1$ ), which is involved in hydrophobic interactions with LMB, Anguinomycin A and KPT8602.

Presented here, alignment of residues interacting with CRM1 inhibitors (Figure 3A) reveals that, in addition to Cys539, residues Lys579 (equivalent to Lys568 in ${ }^{H s} \mathrm{CRM} 1$ ), and Phe583 (equivalent to Phe572 in ${ }^{H s} \mathrm{CRM} 1$ ) are involved in binding of all structurally characterized inhibitors (Figure $3 A)$. Lys579 was shown to play a major role in stabilizing the hydrolyzed lactone ring of LMB, Anguinomycin A and Ratjadone A upon covalent binding to Cys539 by Michael addition [42]. Moreover, its interaction with all structurally characterized KPT-SINE compounds indicates its important role in coordinating the compounds upon binding or stabilizing the non-hydrolyzed compounds after binding to the reactive cysteine [36], [39]. In contrast, the role of Phe583 in inhibitor binding was not investigated up to now by structural studies. Yet, its interaction with all structurally characterized inhibitors demonstrates a major role in inhibitor coordination in CRM1.

Furthermore, the sequence alignment of inhibitor coordinating residues demonstrates the differences in CRM1 binding between the natural inhibitors and the SINE-KPT compounds. LMB, Anguinomycin A and Ratjadone A molecules interact with HEAT repeats $11 \mathrm{~A}$ and $12 \mathrm{~A}$, and partially with HEAT repeat $11 \mathrm{~B}$ as these compounds are able to deeply penetrate the NES-binding cleft. Binding of these inhibitors blocks $60-70 \%$ of surface of the NES-binding cleft (Figure $3 \mathrm{~B}$ ) and occupies 4 out of 5 hydrophobic pockets (Ф0 - Ф3) in which PKI-NES is known to bind (Figure 3D). In contrast, SINE-KPT compounds lack the interaction with the lower part of HEAT repeats 11A and HEAT repeat 12A except for KPT185 (Figures $3 \mathrm{~A}$ and $3 \mathrm{C}$ ) as their binding is centered around 
the reactive cysteine. The superposition of KPT251 in the NES-binding cleft of ${ }^{H s} \mathrm{CRM} 1$ shows that the binding of SINE-KPT compounds blocks 3 hydrophobic pockets (Ф2 - Ф4) leaving $Ф 0$ and $Ф 1$ as not occupied (Figure 3D).

\subsubsection{Compounds C3, C6 and C10 inhibit CRM1 by direct binding in the NES-binding cleft in a Cys528 dependent manner}

In this study we were also aiming to biochemically characterize novel nuclear export inhibiting compounds that have potential to be developed into anti-tumor or anti-viral drugs. Previously, a biosensor based high-throughput screen using the $17 \mathrm{~K}$ ChemBioNet library resulted in the identification of 11 compounds (C1 - C11) as efficient inducers of nuclear export inhibition [46]. Preliminary studies demonstrated these compounds to cause the nuclear accumulation of HIV-1 Rev-NES when tested in several epithelial cancer cell lines. It was also found that the tested compounds act at different rates with an irreversible or reversible mode of inhibition. However, the exact molecular mechanisms of the identified inhibitors remained to be elucidated [46]. Here, we investigated the molecular mechanism of inhibition of three of these by their ability to bind to ${ }^{H s} \mathrm{CRM} 1$. The ability of the compounds $\mathrm{C} 3, \mathrm{C} 6$, and $\mathrm{C} 10$ to dissociate fluorescently labeled PKI $\Phi^{0}$ Leu NES binding to ${ }^{H s} \mathrm{CRM} 1$ was investigated by FP competition assay. All three compounds tested were capable to dissociate CRM1 PKI $\Phi^{0}$ Leu NES binding in a concentration dependent manner (Figure 4A). Compound C6 exhibited the highest binding affinity of the three compounds at a half-maximal inhibitory concentration $\left(I_{50}\right)$ of $4.2 \mu \mathrm{M}$ compared to $\mathrm{C} 10\left(\mathrm{IC}_{50}=40 \mu \mathrm{M}\right)$ and C3 $\left(I_{50}=59 \mu \mathrm{M}\right)$. However, all compounds showed weaker binding than LMB, which exhibits strong binding in the $\mathrm{nM}$ range $\left(\mathrm{IC}_{50}=151 \mathrm{nM}\right)$ (Figure $\left.4 \mathrm{~A}\right)$. The obtained data confirm the nuclear export inhibition caused by $\mathrm{C} 3, \mathrm{C} 6$, and $\mathrm{C} 10$ is by direct binding to CRM1.

To investigate the impact of Cys528 on binding affinity to the NES-binding cleft of ${ }^{H s} \mathrm{CRM} 1$, the FP competition assay was performed by adding these three compounds at concentrations around their $\mathrm{IC}_{50}$ to a reaction mixture containing wt or ${ }^{\mathrm{Hs}} \mathrm{CRM} 1 \mathrm{C} 528 \mathrm{~S}$ mutant. The ability of $\mathrm{C} 6, \mathrm{C} 3$, and C10 to dissociate binding was significantly reduced when Cys528 was changed to serine (Figure 4B). This does not only confirm their binding in the NES-binding cleft, but additionally proves their interaction to be mediated through Cys528. LMB exhibited a weaker binding to ${ }^{H s} \mathrm{CRM} 1 \mathrm{C} 528 \mathrm{~S}$ variant that is comparable to the binding of $\mathrm{C} 3$ and $\mathrm{C} 6$ to the wild type protein. This indicates the cysteine is not strictly required for LMB binding in the human NES-binding cleft, which is in line with similar observation obtained by structural study on LMB binding to T539S ${ }^{5 c} \mathrm{CRM} 1$ [42]. 
A
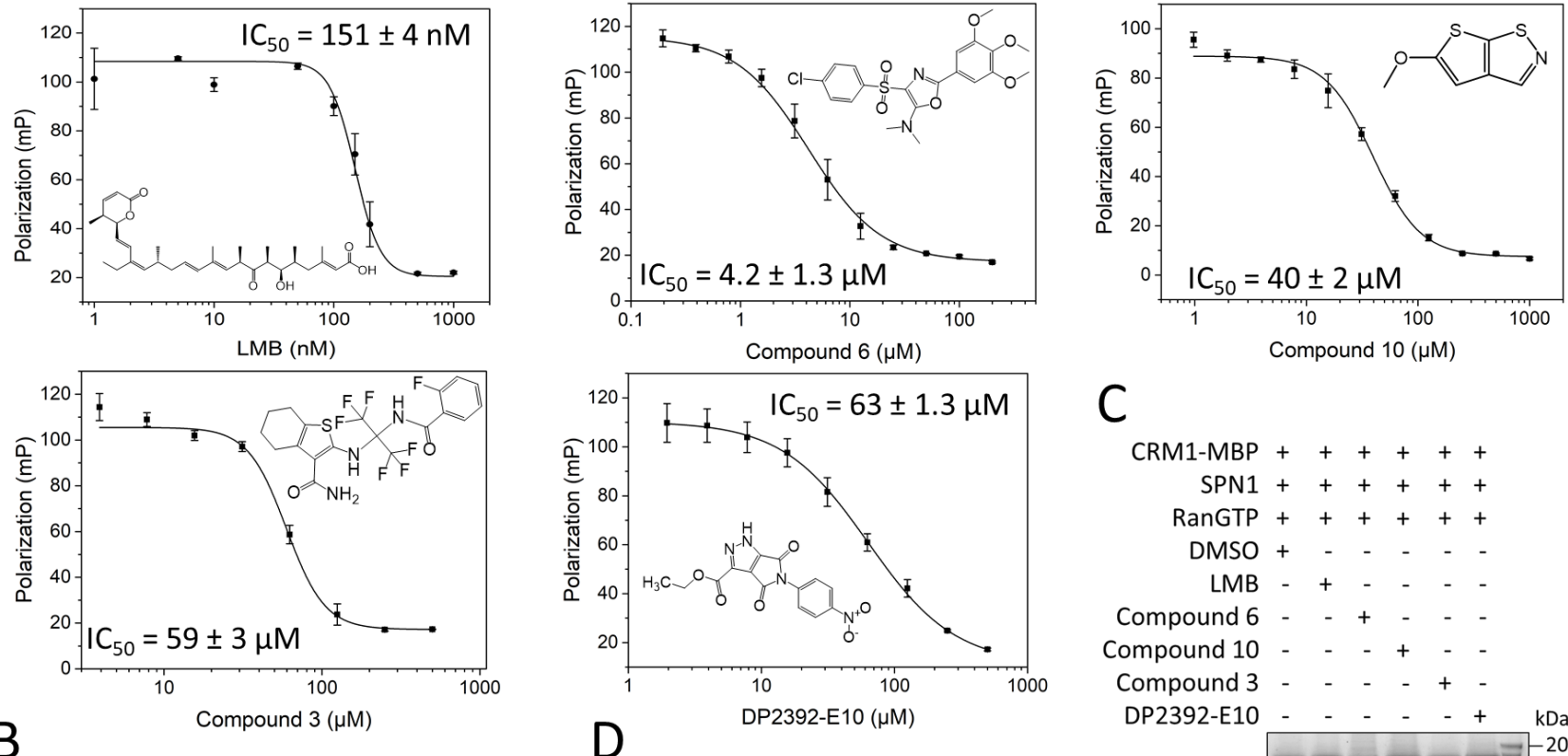

C

CRM1-MBP ++++++

SPN1 ++++++

RanGTP +++++

$\mathrm{DMSO}+-\cdots$

$\mathrm{LMB}-+-\quad-\quad-$

Compound $6-\quad-\quad+\quad-\quad-$

Compound 10 - $-\quad+\quad+\quad-$

Compound 3 - $-c_{-}+$

DP2392-E10 - - - - + $\mathrm{kDa}$
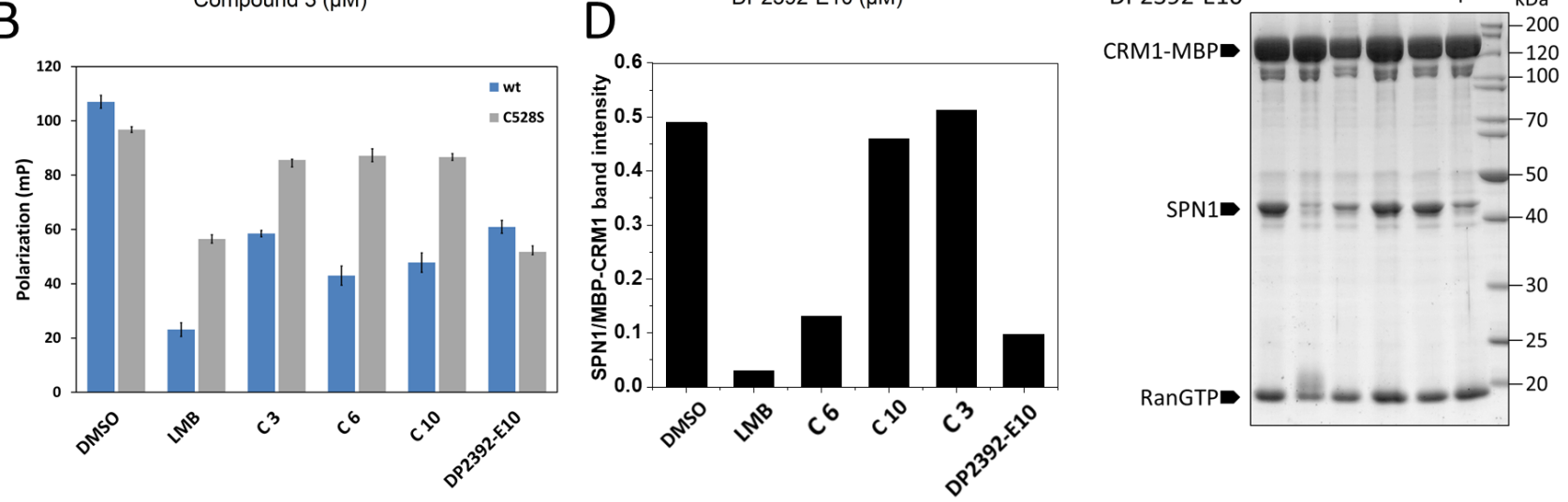

Figure 4: Biochemical analysis of ${ }^{H s} \mathrm{CRM} 1$ inhibitors. (A) Fluorescence polarization competition assay was used to test the inhibition of ${ }^{H s}$ CRM1-NES interaction by leptomycin B (LMB), compounds C6, C10, C3, and DP2392-E10. Chemical drawings of the tested compounds are illustrated in the individual graphs. All tested compounds could dissociate fluorescently labeled PKI $\Phi^{0}$ Leu NES binding to the NES binding-cleft of ${ }^{H s} \mathrm{CRM} 1$ at the indicated binding affinities. (B) FP competition assay of wild type (wt) vs $\mathrm{C} 528 \mathrm{~S}{ }^{\mathrm{Hs}} \mathrm{CRM} 1$. Fluorescence polarization (mP) was measured for each compound (200 nM LMB, $6 \mu \mathrm{M} \mathrm{C6}, 62 \mu \mathrm{M}$ C10, $62 \mu \mathrm{M} \mathrm{C3}$, and $62 \mu \mathrm{M}$ DP2392-E10) added to assay mixture containing wt or $\mathrm{C} 528 \mathrm{~S}{ }^{\mathrm{Hs}} \mathrm{CRM} 1$. Competitive binding of $\mathrm{LMB}, \mathrm{C} 3, \mathrm{C} 6$, and $\mathrm{C} 10$ was significantly reduced when C528S mutant was used, while the inhibitory activity of DP2392-E10 was not significantly affected. Error bars in (A) and (B) illustrate s.d. for three independent measurements. (C) The effect of the tested inhibitors on the stability of ${ }^{H s}$ SPN1-CRM1-RanGTP export complex by pull-down assays with immobilized ${ }^{H s}$ CRM1-MBP. ${ }^{H s} S P N 1$ binding to immobilized ${ }^{H s}$ CRM1-MBP was inhibited by the addition of LMB and weakened by the addition of C6 and DP2392$\mathrm{E} 10$, but not influenced by the addition of $\mathrm{C} 10$ or $\mathrm{C} 3$. The band intensities of ${ }^{\mathrm{Hs} S P N} 1$ and ${ }^{\mathrm{Hs}} \mathrm{CRM} 1-$ MBP were quantified using the ImageJ software [58]. The ratio of ${ }^{H s}$ SPN1 band intensity to ${ }^{H s} \mathrm{CRM} 1-$ MBP band intensity at each reaction is displayed as histogram in (D). 
In addition, we have investigated the capability of these three compounds to dissociate the stable Snurportin1 (SPN1) cargo complex $\left({ }^{H s} \mathrm{CRM1} 1{ }^{H s}\right.$ RanGTP_HsSPN1) by pull-down assays. SPN1 has been shown to interact with the outer surface of CRM1 via the cap binding domain and the Cterminal part of the protein, besides its NES, which renders SPN1 exceptionally stable CRM1 cargo [8], [49], [65]. The pull-down in the presence of C6 exhibited noticeable decrease in SPN1 binding to CRM1 compared to no inhibitor present, but less than LMB that completely abolished SPN1 binding. Compounds $\mathrm{C} 3$ and $\mathrm{C} 10$ used at the same concentrations as compound $\mathrm{C} 6$ did not show a noticeable effect on SPN1 complex stability (Figures 4C and 4D). Increasing concentrations of compounds C3 and C10 to the level corresponding to a molar excess assessed based on their binding affinities could not be performed as it resulted in precipitation of CRM1.

The observed competitive inhibition of the tested compounds against PKI $\Phi^{0}$ Leu NES and SPN1 is dependent on the binding properties of both molecules. As a single peptide, PKI $\Phi^{0}$ Leu NES interaction is mediated only by direct binding in the NES binding cleft while NES-mediated SPN1CRM1 complex formation is supported by additional interactions distant to the NES-binding cleft which further strengthen the stability of the formed complex. Compounds C3 and C10, which exhibited low binding affinities, were able to displace PKI $\Phi^{0}$ Leu NES by direct competition when added at higher concentrations (Figure 4A). However, the dissociation of a more stable CRM1SPN1 complex seems only to be possible with compounds of higher binding affinity like LMB and compound C6 (Figures 4C and 4D).

\subsubsection{DP2392-E10 exhibits a unique binding mode that is not dependent on Cys528}

Another compound that we had chosen to study is DP2392-E10. It was identified by a highthroughput CELAVIEW screening system as a potential inhibitor for the nuclear export of the viral nucleoprotein (NP)-NES3 [66]. A later study revealed that DP2392-E10 reduces the replication of influenza A virus by interfering with CRM1 mediated transport of the vRNP. Further analysis confirmed that this compound binds directly to CRM1 [47]. In addition, DP2392-E10 was predicted by in silico studies to interact with CRM1 in a region outside of the NES-binding cleft near the HEAT9 and HEAT10 repeats. A total of 45 potential small molecule binding pockets were predicted in unliganded CRM1 by molecular modeling and docking simulations. A pocket near HEAT repeats 9 and 10 exhibited the highest docking score and therefore was predicted as the binding location of DP2392-E10 [47]. Here we investigated the molecular basis of DP2392-E10 inhibition of ${ }^{H s} \mathrm{CRM} 1$ by the FP competition and pull-down assays. The results showed that DP2392-E10 can dissociate PKI $\Phi^{0}$ Leu NES bound to ${ }^{H s}$ CRM1 in a concentration dependent 
manner with an $\mathrm{IC}_{50}=63 \mu \mathrm{M}$, which is very close to the binding affinity of $\mathrm{C} 3\left(\mathrm{IC}_{50}=59 \mu \mathrm{M}\right)($ Figure $4 \mathrm{~A})$. Further analysis using $\mathrm{C} 528 \mathrm{~S}{ }^{\mathrm{Hs}} \mathrm{CRM} 1$ revealed that the inhibition potency of DP2392-E10 was not reduced when Cys528 was changed to serine (Figure 4B). This confirms that binding of DP2392-E10 to CRM1 is not mediated via Cys528 and might lie outside of the NES binding cleft as predicted in docking studies.

To strengthen this prediction the ability of DP2392-E10 to dissociate the cargo complex CRM1RanGTP-SPN1 was also examined in a pull-down assay as it has been performed for the compounds C3, C6, and C10. Although C3 and DP2392-E10 have a similar binding affinity, as determined by FP competition assays, only DP2392-E10 was able to reduce SPN1 binding to CRM1 to a level comparable to that observed for C6 (Figure 4C and D). This indicates that these two compounds (C3 and DP2392-E10) differ in their mechanism of inhibition as binding of compound C3 is Cys528 dependent and, despite the similar binding affinity, could not dissociate SPN1 binding when added at the same concentration as DP2392-E10. This additionally supports the prediction that binding of DP2392-E10 occurs outside of the NES binding cleft. Furthermore, predicted binding of DP2392-E10 in the close vicinity of HEAT9 and HEAT10 repeats could influence CRM1 cargo affinity and the opening of the NES-binding cleft by modulating the impact of acidic loop (supplementary figure 2) for which an auto inhibitory effect on NES binding has been reported [62]. In addition, the acidic loop is a key regulator for the conformational transition of CRM1 between the extended and the compact conformation which is directly correlated to cargo binding affinity [60], [61]. This mechanism renders DP2392-E10 a promising compound for developing new classes of CRM1 inhibitors exhibiting different binding mechanism from that of LMB and SINE-compounds. Nevertheless, the obtained data can only confirm the unique binding properties of DP2392-E10 that is not mediated through Cys528. Still, the exact binding location and the chemical mechanism of binding remains to be resolved by future experiments.

\subsubsection{Human and yeast CRM1 reveal different binding affinities towards PKI $\Phi^{0}$ Leu NES}

The comparison of ${ }^{H s} \mathrm{CRM} 1$ and ${ }^{S c} \mathrm{CRM} 1$ reveals their high sequence and structural similarity in the NES-binding cleft region. This however does not guarantee similar binding affinities of NES or inhibitors. Hence, we decided to assess the difference in binding kinetics between ${ }^{H s} \mathrm{CRM} 1$ and ${ }^{S c}$ CRM1 using fluorescently labeled PKI $\Phi^{0}$ Leu NES by FP binding assay (Figure 5A). Wild type ${ }^{H s} \mathrm{CRM} 1$ exhibited high binding affinity $(\mathrm{Kd}=37 \mathrm{nM})$ towards PKI $\Phi^{0}$ Leu NES, while under the same conditions wild type ${ }^{S c} \mathrm{CRM} 1$ exhibited 16 -fold lower binding affinity $(\mathrm{Kd}=585 \mathrm{nM})$. The 
genetic modification of Thr539 to cysteine lead to a minor increase of the affinity, still 12-fold lower than that of ${ }^{H s}$ CRM1 (Figure 5A). Cys528 (Cys539 in modified ${ }^{S c}$ CRM1) is located in the vicinity of $Ф 3$ PKI NES binding pocket (Figure 3D) and was shown to be involved in hydrogenbonding in NES-CRM1 interaction [22]. Nevertheless, the affinity of ${ }^{S c} \mathrm{CRM} 1{ }^{\mathrm{T} 39 \mathrm{C}}$ mutant, is still much lower than that of wild type ${ }^{H s} \mathrm{CRM} 1$.

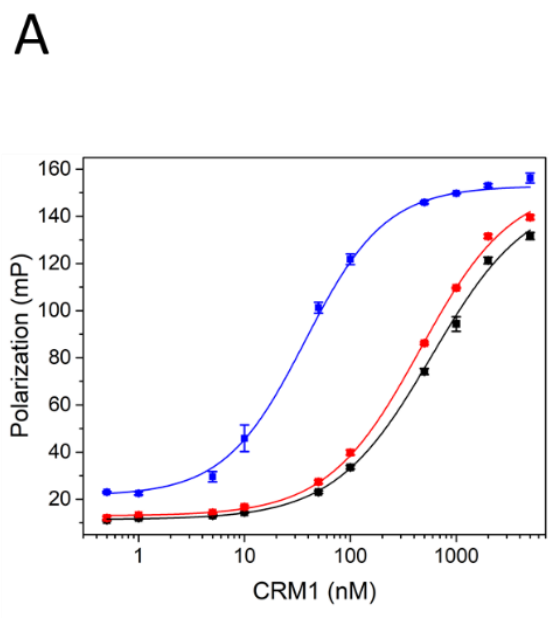

Hs CRM1, $\mathrm{Kd}=37 \pm 1 \mathrm{nM}$

Sc CRM1, Kd = $585 \pm 1 \mathrm{nM}$

T539C Sc CRM1, Kd = $454 \pm 1 \mathrm{nM}$
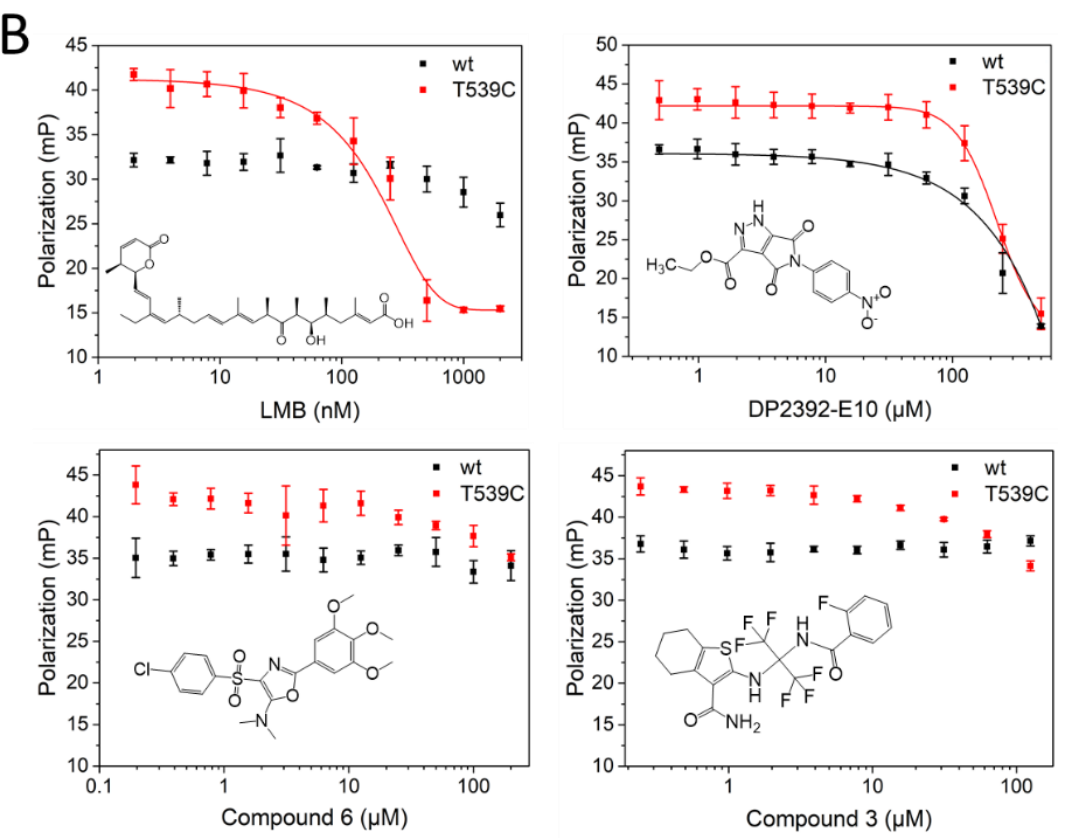

Figure 5: Wild type and $\mathrm{T} 539 \mathrm{C}^{\mathrm{Sc} C R M 1}$ interaction with PKI $\Phi^{\circ}$ Leu NES and CRM1 inhibitors $\mathrm{LMB}, \mathrm{C6}$, C3, and DP2392-E10 measured by fluorescence polarization assay. (A) Comparison of fluorescently labeled PKI $\Phi^{0}$ Leu NES binding to wt ${ }^{H s} \mathrm{CRM} 1$, wt ${ }^{{ }^{S c} \mathrm{CRM}} 1$, and $T 539 \mathrm{C}{ }^{{ }^{S c} \mathrm{CRM} 1}$ in the presence of ${ }^{H s}$ RanGTP ${ }^{1-180, ~ Q 69 L}$ via fluorescence polarization binding assay. Wild type ${ }^{H s} \mathrm{CRM} 1$ binds tightly to PKI $\Phi^{0}$ Leu NES in the low $\mathrm{nM}$ range, while wild type ${ }^{S C} \mathrm{CRM} 1$ exhibited weaker binding of around 16folds. Introducing the $\mathrm{T} 539 \mathrm{C}$ mutation increased the affinity slightly to be 12 -fold less compared to that of ${ }^{H s}$ CRM1 (B) Fluorescence polarization competition assay for inhibition of ${ }^{S C} \mathrm{CRM} 1 \mathrm{NES}$ interaction by LMB, C6, C3, and DP2392-E10. DP2392-E10 could dissociate PKI $\Phi^{0}$ Leu NES interaction when added to high concertation to the wt ${ }^{S c} C R M 1$ and $T 539 C$ mutant. Introducing the T539C mutation resulted in the binding of LMB but not C6 and C3. Error bars illustrate s.d. for three independent measurements.

Interestingly, despite distal binding affinities, the residues coordinating PKI NES in ${ }^{H s} \mathrm{CRM} 1$ and ${ }^{5 c}$ CRM1 1 are identical (Figure 6A). Furthermore, the NES binding cleft seems to be evolutionary conserved from yeast to human (Figure 6B) [8]. This indicates that the difference in binding affinity arises from differences in regions that are not directly involved in NES binding. CRM1 cargo affinity has been shown to be strongly influenced by the overall conformational transition 
of CRM1 between the extended and the compact conformations, which is regulated by structural elements outside the NES binding cleft. In addition, the compact conformation in which the NES cleft is open, is further stabilized by massive interactions between the $\mathrm{N}$ - and the $\mathrm{C}$ - terminal regions [60], [61]. In fact, ${ }^{H s} \mathrm{CRM} 1$ and ${ }^{S c} \mathrm{CRM} 1$ share low sequence identity in the regions outside the NES-binding cleft, especially the C- terminal domain (Figure 6A). The lack of sequence conservation outside the NES-binding cleft seems to have the major influence on CRM1 flexibility and its overall conformation. Previous single particle cryo EM studies revealed that ${ }^{H s}$ CRM1 exhibit higher structural flexibility than Chaetomium thermophilum CRM1 $\left({ }^{C t}\right.$ CRM1, 50.3\% sequence identity to ${ }^{H s} \mathrm{CRM} 1$ ) [60]. It also showed that 2/3 of ${ }^{S c} \mathrm{CRM} 1$ particles accommodate the extended conformation in solution, while only half of the human particles retained this conformation [60], [61]. Taken together, sequence differences in regions outside the NES-binding cleft seem to have a significant influence on CRM1 conformation which in turn is reflected by the variation in CRM1-NES binding affinity.

\subsubsection{Thr539Cys ${ }^{S c}$ CRM1 mutant binds strongly LMB but not compounds $C 3$ and $C 6$.}

After observing the different PKI-NES binding kinetics of HsCRM1 and ${ }^{\mathrm{SC} C R M} 1{ }^{\mathrm{T} 39 \mathrm{C}}$ we addressed the question on how the Thr539Cys mutation affects ${ }^{5 c}$ CRM1 interaction with LMB, C3, C6, and DP2392-E10 by FP competition assay (Figure 5B). In the absence of Cys539 neither LMB nor C3 or C6 could bind to ${ }^{S c} \mathrm{CRM} 1$. Conversely, DP2392-E10, as its binding is not dependent on the reactive cysteine (Figure $4 \mathrm{~B}$ ), was able to bind to the ${ }^{5 c} \mathrm{CRM} 1$ at higher concentrations. As expected, modification of Thr539 to cysteine could restore LMB binding to a similar level as observed for wild type ${ }^{H s}$ CRM1. However, C6 and C3 did not show any significant change in binding affinity even when the cysteine was introduced into the ${ }^{{ }^{C} C} \mathrm{CRM} 1{ }^{\mathrm{T} 339 C}$ NES-binding cleft, although their binding to ${ }^{H s} \mathrm{CRM} 1$ was shown to be Cys528 dependent. Only a very low tendency of binding was observed when both compounds were added at a very high concentration (Figure 5B). Taken together, the obtained data permit the conclusion that despite the structural similarity of ${ }^{S c} \mathrm{CRM} 1$ and ${ }^{H s} \mathrm{CRM} 1$ and the high sequence conservation of their NES-binding cleft, both transport receptors demonstrate different binding kinetics towards identical inhibitors. This proves that both cargo and inhibitor binding are significantly influenced by the overall structural arrangement of CRM1, which in turn varies in correlation to the amino acids sequence of regions outside the NES binding cleft. 
A

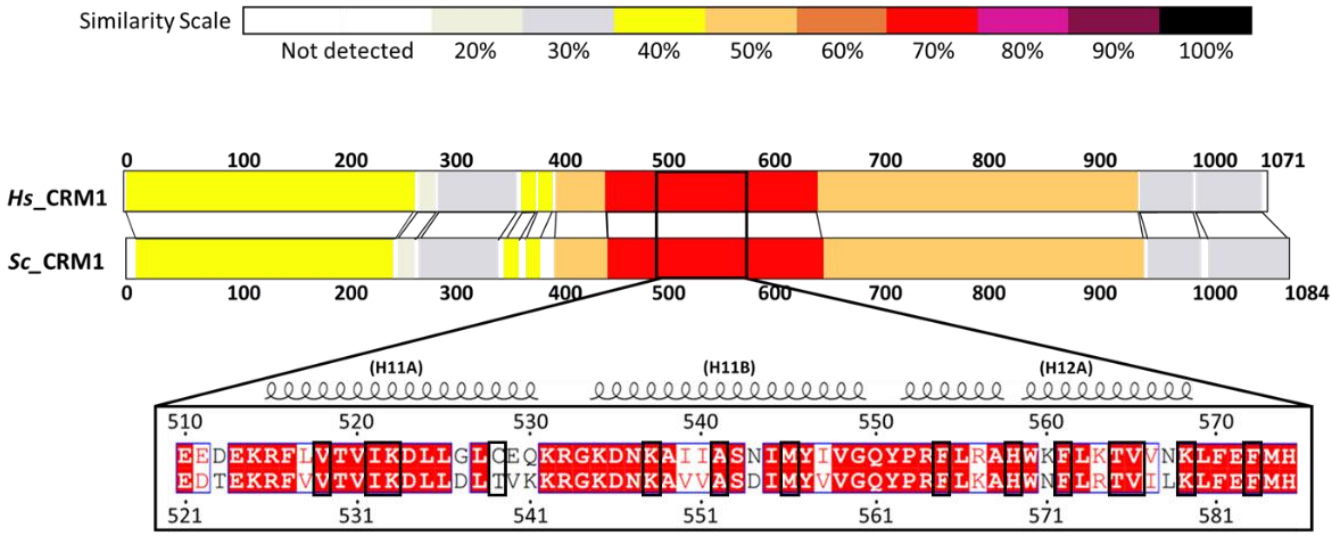

B

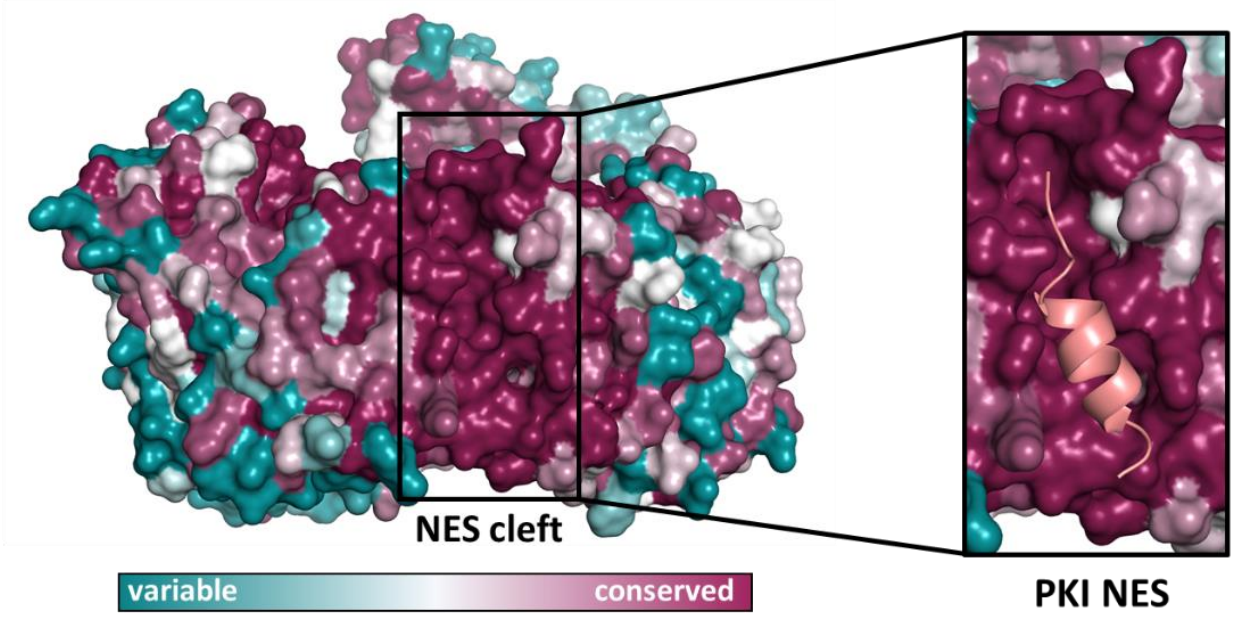

Figure 6: CRM1 sequence alignment and conservation from yeast to human. (A) Schematic representation of amino acid sequence alignments between human and Saccharomyces cerevisiae CRM1. Regions of homology were determined using SIM alignment tool (https://web.expasy.org/sim/) and illustrated using LalnView [67]. Both proteins have in total $47 \%$ sequence identity. The region spans from approximately amino acids $450-640$, which include the NES-binding cleft, exhibit high sequence identity (75-80\%). Residues contacting PKI-NES are marked with black boxes. (B) Sequence conservation of CRM1 in the structural context of LMB-bound human CRM1. CRM1 is depicted in a surface mode and colored according to sequence conservation from invariant (deep purple) to variable (deep teal). PKI NES (PDB ID 3NBY) was aligned in the NESbinding cleft and depicted as cartoon (right). CRM1 shows the conservation of NES-binding cleft from yeast to human. PKI-NES bind in the highly conserved region of the NES-binding cleft. Conservation coloring is based on a multiple sequence alignment of CRM1 from $H$. sapiens, $M$. musculus, $R$. norvegicus, S. pombe, S. cerevisiae, and C. thermophilum. 


\subsection{Conclusion}

Cellular mislocalization of tumor suppressor proteins and oncoproteins mediated by CRM1 leads to cancer initiation, progression, and interferes with cancer treatment. In addition, CRM1 dependent export is co-opted by viruses for the nuclear export of their RNAs and RNPs at different stages of replication which mediate and establish viral infection. Since the identification of LMB as a toxic CRM1 inhibitor, several inhibitors targeting CRM1 have been developed utilizing structure-based drug design. This approach turned out to be an essential tool for defining the molecular basis of CRM1 inhibition and design of less toxic drugs. The structural analysis of CRM1inhibitor complexes was performed using CRM1 from the yeast Saccharomyces cerevisiae as it crystallized more easily than the human orthologue and because of the high sequence similarity in the NES-binding cleft of both organisms. Previous analysis revealed that LMB as well as other synthetic SINE-KPT compounds bind to CRM1 via the reactive Cys528 by Michael addition. In this study we used ${ }^{H s} \mathrm{CRM} 1$ for the biochemical characterization of potential CRM1 inhibitors: C3, C6, C10, and DP2392-E10. C3, C6, and C10 demonstrated Cys528 dependent binding to CRM1 with different binding affinities and most likely a different mode of inhibition than LMB and SINEcompounds. We were also able to show that DP2392-E10 binding is not mediated through the reactive cystine. Moreover, the capability of DP2392-E10 to dissociate the stable SPN1 export complex, despite its low binding affinity, strongly support the previously predicted binding outside the NES-binding cleft. Our biochemical analysis renders these compounds, in particular DP2392-E10, as potential candidates for the development of a new class of CRM1- inhibitors.

Despite the structural and sequence similarity of the NES-binding cleft region in ${ }^{H s} \mathrm{CRM} 1$ and ${ }^{S C} C R M 1$, they exhibited different binding properties towards PKI $\Phi^{\circ}$ Leu NES as well as to the compounds $\mathrm{C} 3$ and $\mathrm{C} 6$. We found that both wild type and Thr539Cys variant of ${ }^{\mathrm{Sc}} \mathrm{CRM} 1$ exhibit weaker binding towards PKI $\Phi^{0}$ Leu NES and no or extremely low binding towards the compounds C3 and C6. This indicates that the amino acid differences outside the NES-binding cleft have a strong influence of CRM1-cargo/inhibitor affinity, which is strongly dependent on CRM1 conformational transition regulated by structural elements outside the NES-binding cleft. Our data indicate that ${ }^{S C} \mathrm{CRM} 1$ - based molecular and potentially structural analysis of CRM1 inhibitors is limited to certain class of inhibitors exhibiting high binding affinity and/or irreversible binding. Hence, ${ }^{H s}$ CRM1 should be used for structure-based development of novel drugs. Our findings further indicate for the importance of ${ }^{H s} \mathrm{CRM} 1$ for the accurate determination of inhibitors potency which is a very important and defining value for their efficacy. 


\subsection{Associated Content}

\section{Supporting Information}

Additional figure illustrating the binding of PKI $\Phi^{0}$ Leu NES to ${ }^{H s} \mathrm{CRM} 1$ variants as well as a figure demonstrating the overall structure of ${ }^{H s} \mathrm{CRM} 1-{ }^{H s}$ RanGTP-LMB complex.

\section{Accession Codes}

PDB code for Human CRM1-RanGTP in complex with Leptomycin B is 6TVO

\subsection{Author Information}

\section{Corresponding Author}

*E.mail: rficner@uni-goettingen.de. Phone: +49 (551) 3928618

\section{Author Contributions}

Experimental design (A.S., R.F.), molecular cloning (A.S.), protein expression and purification (A.S.), crystallization of protein complexes (A.S.), data collection and processing (A.S.), structure determination and refinement (A.S., P.N.), crystal structure analysis (A.S., P.N.), Fluorescence polarization binding and competition assays (A.S.), Pull-down assay (A.S.) data analysis and discussion (A.S., P.N., A.D., R.F.), preparation of figures/tables (A.S.), writing original draft (A.S.), writing - review and editing (A.S., P.N., A.D., R.F.), supervision, project leader (R.F.).

\section{Funding Sources}

This work was supported with funding by the DFG SFB860 (TP B08) and the University of Göttingen.

\section{Notes}

The authors declare no competing financial interest.

\subsection{Acknowledgement}

The synchrotron X-ray diffraction data was collected at beamline P14 operated by EMBL Hamburg at the PETRA III storage ring (DESY, Hamburg, Germany). We would like to thank Isabel Bento for the assistance in using the beamline. Furthermore, we are very grateful for Dr. Thomas Monecke for his outstanding assistance in initial experimental design, and data collection. We are grateful to Roshan Perera (Department of Developmental Biochemistry, University of Göttingen) for his assistance in cloning yeast CRM1. 


\subsection{Abbreviations}

NPCs, nuclear pore complexes; CRM1, chromosome region maintenance 1; vRNP, viral ribonucleoproteins; NES, nuclear export signals; LMB, Leptomycin B; PKI, protein kinase A inhibitor; FP, fluorescence polarization; SINE, selective inhibitors of nuclear export; IPTG, Isopropyl $\beta$-D1-thiogalactopyranoside; OD, optical density; MBP, maltose-binding protein; $\mathrm{His}_{6}$, hexa histidine-tag 


\subsection{Supplementary Information}

\section{Characterization of inhibition reveals distinctive properties for human and Saccharomyces cerevisiae CRM1}

\section{Alaa Shaikhqasem, Achim Dickmanns, Piotr Neumann and Ralf Ficner*}

Department of Molecular Structural Biology, Institute of Microbiology and Genetics, GZMB, Georg-August-University Göttingen, 37077 Göttingen, Germany

Additional figure illustrating the binding of PKI $\Phi^{0}$ Leu NES to ${ }^{H s} C R M 1$ variants as well as a figure demonstrating the overall structure of ${ }^{H s} \mathrm{CRM} 1-{ }^{H s}$ RanGTP-LMB complex.

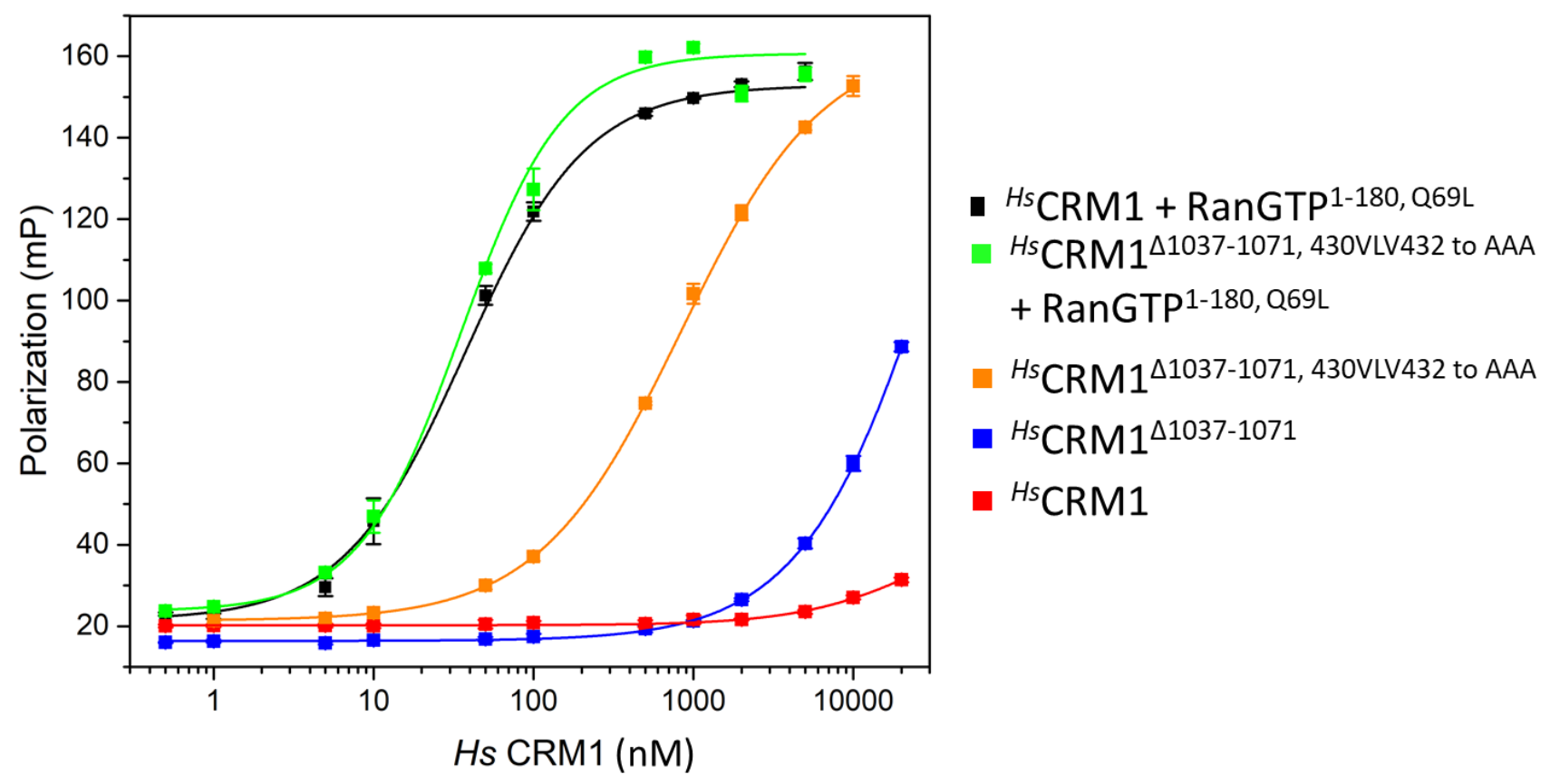

Supplementary Figure 1: Fluorescently labeled PKI $\Phi^{0}$ Leu NES binding to ${ }^{H s} \mathrm{CRM} 1$ variants and fulllength wild type in the presence or absence of ${ }^{H s}$ RanGTP ${ }^{1-180}$, Q69L by fluorescence polarization binding assay. Wild type ${ }^{H s} \mathrm{CRM} 1$ as well as the C-terminal helix-acidic loop mutant reveal a very strong binding to the PKI $\Phi^{\circ}$ Leu NES in the presence of RanGTP. Truncating the C-terminal helix of ${ }^{H s}$ CRM1 ( $\left.\triangle 1037-1071\right)$ increased the affinity to the PKI $\Phi^{0}$ Leu NES in the absence of RanGTP. Furthermore, a combination of C-terminal helix truncation and HEAT9 loop variant $\left({ }^{430} \mathrm{VLV} \mathrm{V}^{432}\right.$ mutated to $A A A$ ) resulted in a higher increase of ${ }^{H s}$ CRM1 affinity to PKI NES in the absence of RanGTP. Error bars illustrate s.d. for three independent measurements. 


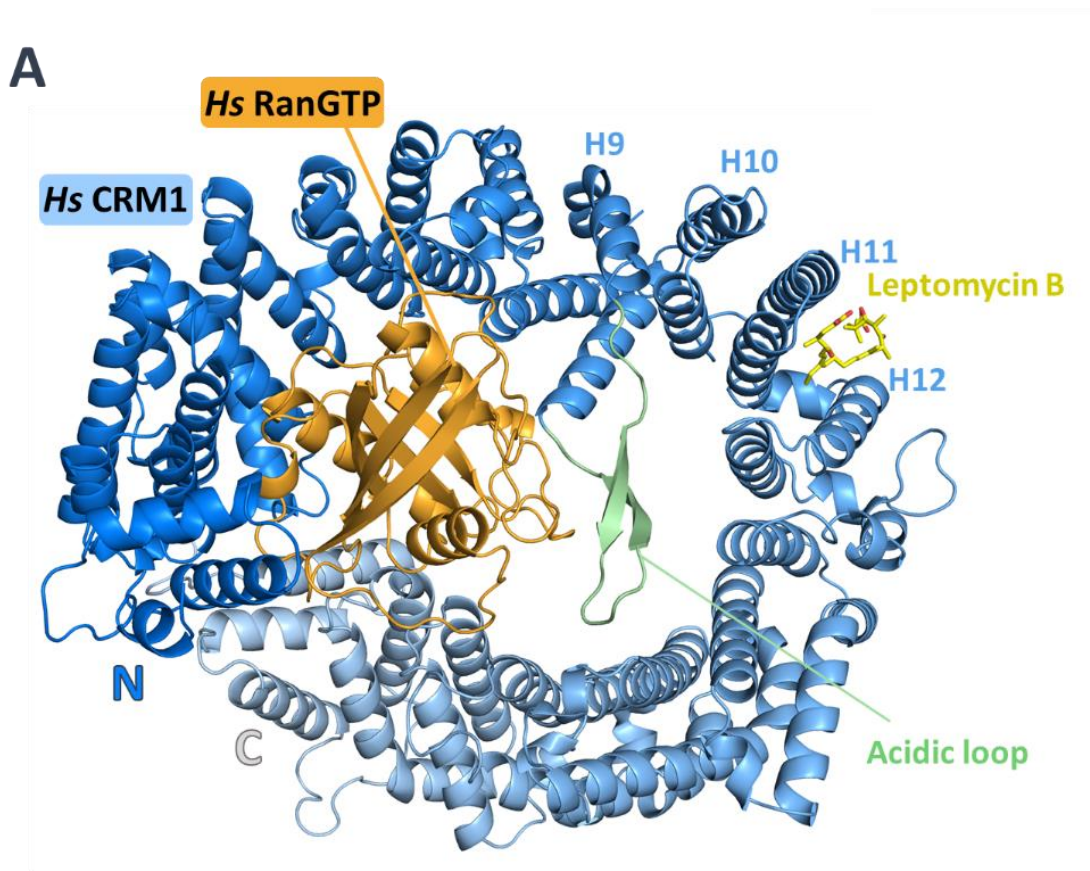

\section{B}

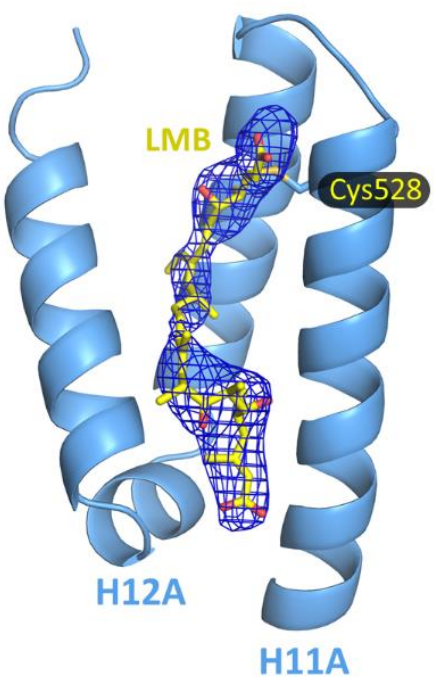

Supplementary Figure 2: (A) Crystal structure of ${ }^{H s} \mathrm{CRM} 1-{ }^{H s}$ RanGTP-LMB complex illustrated in cartoon representation. CRM1 is gradient colored from the $\mathrm{N}$-terminus (marine) to the $\mathrm{C}$ terminus (white), while Ran is shown in light orange color. The acidic loop adopting the seatbelt conformation is shown in pale green. LMB bound in the nuclear export signal (NES)-binding cleft, formed by HEAT helices $11 \mathrm{~A}$ and $12 \mathrm{~A}$, is shown in stick mode. (B) LMB is defined by a Polder mFo-DFc omit map (blue mesh) contoured at a sigma level 3.0. 


\subsection{References}

[1] K. Stade, C. S. Ford, C. Guthrie, and K. Weis, "Exportin 1 (Crm1p) is an essential nuclear export factor.," Cell, vol. 90, no. 6, pp. 1041-50, Sep. 1997.

[2] U. Kutay, E. Izaurralde, F. R. Bischoff, I. W. Mattaj, and D. Görlich, "Dominant-negative mutants of importin-beta block multiple pathways of import and export through the nuclear pore complex.," EMBO J., vol. 16, no. 6, pp. 1153-63, Mar. 1997.

[3] E. J. Tran and S. R. Wente, "Dynamic Nuclear Pore Complexes: Life on the Edge," Cell, vol. 125, no. 6. pp. 1041-1053, Jun-2006.

[4] A. G. Cook and E. Conti, "Nuclear export complexes in the frame," Current Opinion in Structural Biology, vol. 20, no. 2. pp. 247-252, Apr-2010.

[5] H. B. Schmidt and D. Görlich, "Transport Selectivity of Nuclear Pores, Phase Separation, and Membraneless Organelles.," Trends Biochem. Sci., vol. 41, no. 1, pp. 46-61, Jan. 2016.

[6] D. Görlich and I. W. Mattaj, "Nucleocytoplasmic transport.," Science, vol. 271, no. 5255, pp. 1513-8, Mar. 1996.

[7] K. S. Ullman, M. A. Powers, and D. J. Forbes, "Nuclear export receptors: from importin to exportin.," Cell, vol. 90, no. 6, pp. 967-70, Sep. 1997.

[8] T. Monecke, A. Dickmanns, and R. Ficner, "Allosteric control of the exportin CRM1 unraveled by crystal structure analysis," FEBS J., vol. 281, no. 18, pp. 4179-4194, Sep. 2014.

[9] K. Kırlı et al., "A deep proteomics perspective on CRM1-mediated nuclear export and nucleocytoplasmic partitioning," Elife, vol. 4, no. DECEMBER2015, Dec. 2015.

[10] R. Hill, B. Cautain, N. De Pedro, and W. Link, "Targeting nucleocytoplasmic transport in cancer therapy.," Oncotarget, vol. 5, no. 1, pp. 11-28, Jan. 2014.

[11] R. S. Faustino, T. J. Nelson, A. Terzic, and C. Perez-Terzic, "Nuclear transport: target for therapy.," Clin. Pharmacol. Ther., vol. 81, no. 6, pp. 880-6, Jun. 2007.

[12] J. G. Turner, J. Dawson, and D. M. Sullivan, "Nuclear export of proteins and drug resistance in cancer.," Biochem. Pharmacol., vol. 83, no. 8, pp. 1021-32, Apr. 2012.

[13] P. J. van der Watt and V. D. Leaner, "Regulation of Crm1 expression in cancer cells," 2010, pp. A33-A33.

[14] K. Nakano and T. Watanabe, "HTLV-1 Rex Tunes the Cellular Environment Favorable for Viral Replication.," Viruses, vol. 8, no. 3, p. 58, Feb. 2016.

[15] M. Nagai-Fukataki, T. Ohashi, I. Hashimoto, T. Kimura, Y. Hakata, and H. Shida, "Nuclear and cytoplasmic effects of human CRM1 on HIV-1 production in rat cells.," Genes Cells, vol. 16, no. 2, pp. 203-16, Feb. 2011.

[16] S. Cao et al., "A nuclear export signal in the matrix protein of Influenza A virus is required for efficient virus replication.," J. Virol., vol. 86, no. 9, pp. 4883-91, May 2012.

[17] C. Mathew and R. Ghildyal, "CRM1 inhibitors for antiviral therapy," Frontiers in Microbiology. 2017. 
[18] A. Dickmanns, T. Monecke, and R. Ficner, Structural Basis of Targeting the Exportin CRM1 in Cancer, vol. 4, no. 3. 2015.

[19] S. Kosugi, M. Hasebe, M. Tomita, and H. Yanagawa, "Nuclear export signal consensus sequences defined using a localization-based yeast selection system," Traffic, vol. 9, no. 12, pp. 2053-2062, 2008.

[20] D. Xu, A. Farmer, G. Collett, N. V. Grishin, and Y. M. Chook, "Sequence and structural analyses of nuclear export signals in the NESdb database," Mol. Biol. Cell, vol. 23, no. 18, pp. 3677-3693, Sep. 2012.

[21] H. Y. J. Fung, S. C. Fu, C. A. Brautigam, and Y. M. Chook, "Structural determinants of nuclear export signal orientation in binding to exportin CRM1," Elife, vol. 4, no. September 2015, pp. 119, 2015.

[22] T. Güttler et al., "NES consensus redefined by structures of PKI-type and Rev-type nuclear export signals bound to CRM1," Nat. Struct. Mol. Biol., vol. 17, no. 11, pp. 1367-1376, 2010.

[23] N. Kudo et al., "Leptomycin B inactivates CRM1/exportin 1 by covalent modification at a cysteine residue in the central conserved region," Proc. Natl. Acad. Sci., 1999.

[24] K. Nishi, M. Yoshida, D. Fujiwara, M. Nishikawa, S. Horinouchi, and T. Beppu, "Leptomycin B targets a regulatory cascade of $\mathrm{crm} 1$, a fission yeast nuclear protein, involved in control of higher order chromosome structure and gene expression.," J. Biol. Chem., vol. 269, no. 9, pp. 6320-4, Mar. 1994.

[25] N. Kudo et al., "Leptomycin B inhibition of signal-mediated nuclear export by direct binding to CRM1," Exp. Cell Res., vol. 242, no. 2, pp. 540-547, Aug. 1998.

[26] E. S. Newlands, G. J. Rustin, and M. H. Brampton, "Phase I trial of elactocin.," Br. J. Cancer, vol. 74, no. 4, pp. 648-9, Aug. 1996.

[27] S. Tamura, N. Shimizu, K. Fujiwara, M. Kaneko, T. Kimura, and N. Murakami, "Bioisostere of valtrate, anti-HIV principle by inhibition for nuclear export of Rev.," Bioorg. Med. Chem. Lett., vol. 20, no. 7, pp. 2159-62, Apr. 2010.

[28] T. R. Kau et al., "A chemical genetic screen identifies inhibitors of regulated nuclear export of a Forkhead transcription factor in PTEN-deficient tumor cells.," Cancer Cell, vol. 4, no. 6, pp. 46376, Dec. 2003.

[29] Y. Lu et al., "15-Deoxy- $\Delta$-Prostaglandin J2 Modulates Lipopolysaccharide- Induced Chemokine Expression by Blocking Nuclear Factor-KB Activation via Peroxisome Proliferator Activated Receptor- $\gamma$-Independent Mechanism in Renal Tubular Epithelial Cells," Nephron - Exp. Nephrol., vol. 123, no. 1-2, pp. 1-10, Aug. 2013.

[30] X. Liu, Y. Chong, H. Liu, Y. Han, and M. Niu, "Novel reversible selective inhibitor of CRM1 for targeted therapy in ovarian cancer.," J. Ovarian Res., vol. 8, p. 35, Jun. 2015.

[31] D. Daelemans et al., "A synthetic HIV-1 Rev inhibitor interfering with the CRM1-mediated nuclear export.," Proc. Natl. Acad. Sci. U. S. A., vol. 99, no. 22, pp. 14440-5, Oct. 2002.

[32] S. Bonazzi et al., "Anguinomycins and Derivatives: Total Syntheses, Modeling, and Biological Evaluation of the Inhibition of Nucleocytoplasmic Transport," J. Am. Chem. Soc., vol. 132, no. 4, 
pp. 1432-1442, Feb. 2010.

[33] K. Sakakibara et al., "CBS9106 is a novel reversible oral CRM1 inhibitor with CRM1 degrading activity," Blood, vol. 118, no. 14, pp. 3922-3931, Oct. 2011.

[34] M. Niu et al., "Piperlongumine is a novel nuclear export inhibitor with potent anticancer activity.," Chem. Biol. Interact., vol. 237, pp. 66-72, Jul. 2015.

[35] G. L. Gravina, W. Senapedis, D. McCauley, E. Baloglu, S. Shacham, and C. Festuccia, "Nucleocytoplasmic transport as a therapeutic target of cancer," Journal of Hematology and Oncology, vol. 7, no. 1. BioMed Central Ltd., 2014.

[36] J. Etchin et al., "Antileukemic activity of nuclear export inhibitors that spare normal hematopoietic cells," Leukemia, 2013.

[37] Q. Sun, X. Chen, Q. Zhou, E. Burstein, S. Yang, and D. Jia, "Inhibiting cancer cell hallmark features through nuclear export inhibition," Signal Transduction and Targeted Therapy, vol. 1. Springer Nature, 2016.

[38] Y. Y. Syed, "Selinexor: First Global Approval," Drugs, Aug. 2019.

[39] R. Lapalombella et al., "Selective inhibitors of nuclear export show that CRM1/XPO1 is a target in chronic lymphocytic leukemia," Blood, vol. 120, no. 23, pp. 4621-4634, Nov. 2012.

[40] J. D. Haines et al., "Nuclear export inhibitors avert progression in preclinical models of inflammatory demyelination," Nat. Neurosci., vol. 18, no. 4, pp. 511-520, Apr. 2015.

[41] Z. A. Hing et al., "Next-generation XPO1 inhibitor shows improved efficacy and in vivo tolerability in hematological malignancies," Leukemia, 2016.

[42] Q. Sun et al., "Nuclear export inhibition through covalent conjugation and hydrolysis of Leptomycin B by CRM1," Proc. Natl. Acad. Sci., vol. 110, no. 4, pp. 1303-1308, 2013.

[43] O. Kalid, D. Toledo Warshaviak, S. Shechter, W. Sherman, and S. Shacham, "Consensus Induced Fit Docking (cIFD): methodology, validation, and application to the discovery of novel Crm1 inhibitors.," J. Comput. Aided. Mol. Des., vol. 26, no. 11, pp. 1217-28, Nov. 2012.

[44] S. C. Fu, H. Y. J. Fung, T. Caǧatay, J. Baumhardt, and Y. M. Chook, "Correlation of CRM1-NES affinity with nuclear export activity," Mol. Biol. Cell, vol. 29, no. 17, pp. 2037-2044, Aug. 2018.

[45] K. T. Nguyen, M. P. Holloway, and R. A. Altura, "The CRM1 nuclear export protein in normal development and disease.," Int. J. Biochem. Mol. Biol., vol. 3, no. 2, pp. 137-51, 2012.

[46] V. Fetz, S. K. Knauer, C. Bier, J. P. von Kries, and R. H. Stauber, "Translocation biosensors ? Cellular system integrators to dissect CRM1-dependent nuclear export by chemicogenomics," Sensors, vol. 9, no. 7, pp. 5423-5445, Jul. 2009.

[47] N. Chutiwitoonchai et al., "Inhibition of CRM1-mediated nuclear export of influenza A nucleoprotein and nuclear export protein as a novel target for antiviral drug development," Virology, vol. 507, no. April, pp. 32-39, Jul. 2017.

[48] A. Strasser et al., "Purification, crystallization and preliminary crystallographic data of the m3G cap-binding domain of human snRNP import factor snurportin 1.," Acta Crystallogr. D. Biol. Crystallogr., vol. 60, no. Pt 9, pp. 1628-31, Sep. 2004. 
[49] T. Monecke, T. Güttler, P. Neumann, A. Dickmanns, D. Görlich, and R. Ficner, "Crystal structure of the nuclear export receptor CRM1 in complex with snurportin1 and RanGTP," Science (80-. )., vol. 324, no. 5930, pp. 1087-1091, May 2009.

[50] S. A. Port et al., "Structural and Functional Characterization of CRM1-Nup214 Interactions Reveals Multiple FG-Binding Sites Involved in Nuclear Export," Cell Rep., vol. 13, no. 4, pp. 690702, 2015.

[51] F. Gorrec, "research papers The MORPHEUS protein crystallization screen research papers," October, pp. 1035-1042, 2009.

[52] W. Kabsch, "Integration, scaling, space-group assignment and post-refinement," Acta Crystallogr. Sect. D Biol. Crystallogr., vol. 66, no. 2, pp. 133-144, 2010.

[53] A. J. McCoy, R. W. Grosse-Kunstleve, P. D. Adams, M. D. Winn, L. C. Storoni, and R. J. Read, “Phaser crystallographic software," J. Appl. Crystallogr., vol. 40, no. 4, pp. 658-674, Jul. 2007.

[54] P. Emsley and K. Cowtan, "Coot: Model-building tools for molecular graphics," Acta Crystallogr. Sect. D Biol. Crystallogr., vol. 60, no. 12 I, pp. 2126-2132, Dec. 2004.

[55] P. D. Adams et al., "PHENIX: A comprehensive Python-based system for macromolecular structure solution," Acta Crystallogr. Sect. D Biol. Crystallogr., vol. 66, no. 2, pp. 213-221, 2010.

[56] D. Liebschner et al., "Polder maps: improving OMIT maps by excluding bulk solvent.," Acta Crystallogr. Sect. D, Struct. Biol., vol. 73, no. Pt 2, pp. 148-157, 2017.

[57] Laskowski, R A. et al., "LigPlot+: Multiple ligand-protein interaction diagrams for drug discovery," J. Chem. Inf. Model., 2011.

[58] C. A. Schneider, W. S. Rasband, and K. W. Eliceiri, "NIH Image to ImageJ: 25 years of image analysis," Nature Methods, vol. 9, no. 7. pp. 671-675, Jul-2012.

[59] E. Seifert, "OriginPro 9.1: Scientific data analysis and graphing software - Software review," Journal of Chemical Information and Modeling, vol. 54, no. 5. American Chemical Society, p. 1552, 28-Apr-2014.

[60] N. Dölker et al., "Structural determinants and mechanism of mammalian CRM1 allostery," Structure, vol. 21, no. 8, pp. 1350-1360, Aug. 2013.

[61] T. Monecke et al., "Structural basis for cooperativity of CRM1 export complex formation.," Proc. Natl. Acad. Sci. U. S. A., vol. 110, no. 3, pp. 960-5, Jan. 2013.

[62] M. Koyama and Y. Matsuura, "An allosteric mechanism to displace nuclear export cargo from CRM1 and RanGTP by RanBP1," EMBO J., 2010.

[63] A. M. Fox, D. Ciziene, S. H. McLaughlin, and M. Stewart, "Electrostatic interactions involving the extreme $\mathrm{C}$ terminus of nuclear export factor CRM1 modulate its affinity for cargo," J. Biol. Chem., vol. 286, no. 33, pp. 29325-29335, 2011.

[64] A. Y. Wang and H. Liu, "The past, present, and future of CRM1/XPO1 inhibitors," Stem Cell Investig., 2019.

[65] X. Dong et al., "Structural basis for leucine-rich nuclear export signal recognition by CRM1," Nature, vol. 458, no. 7242, pp. 1136-1141, Apr. 2009. 
[66] M. Kakisaka, T. Mano, and Y. Aida, "A high-throughput screening system targeting the nuclear export pathway via the third nuclear export signal of influenza A virus nucleoprotein," Virus Research, vol. 217. Elsevier B.V., pp. 23-31, 02-Jun-2016.

[67] L. Duret, E. Gasteiger, and G. Perrière, "LALNVIEW: a graphical viewer for pairwise sequence alignments.," Comput. Appl. Biosci., vol. 12, no. 6, pp. 507-10, Dec. 1996. 
Manuscript to be submitted

\section{Chapter 3: Molecular docking of novel nuclear export inhibitors reveals an allosteric mechanism of human CRM1 inhibition}

\section{Alaa Shaikhqasem and Ralf Ficner*}

Department of Molecular Structural Biology, Institute of Microbiology and Genetics, GZMB, Georg-August-University Göttingen, 37077 Göttingen, Germany; alaa.shaikqasem@unigoettingen.de (A.S.)

* To whom correspondence should be addressed: Ralf Ficner, Department of Molecular Structural Biology, Institute for Microbiology and Genetics, Georg-August-University Goettingen, 37077 Goettingen, Germany; rficner@uni-goettingen.de 


\section{Abstract}

Cellular mislocalization of oncoproteins and tumor suppressor proteins mediated by Chromosome Region Maintenance 1 (CRM1) leads to cancer initiation and progression. In addition, CRM1 is utilized in several viral diseases like HIV and influenza for the nuclear export of viral RNAs and ribonucleoproteins. This renders CRM1 a particularly interesting target for antitumor and anti-viral drug development. Recently we have investigated the molecular basis of CRM1 inhibition by four novel nuclear export inhibitors: C3, C6, C10, and DP2392-E10. Our results revealed that $\mathrm{C} 3, \mathrm{C} 6$, and $\mathrm{C} 10$ demonstrate Cys528 dependent binding which leads to the dissociation of CRM1 interaction with the nuclear export signal (NES). Whereas, DP2392-E10 exhibited a unique binding behavior that is not dependent on the reactive cysteine. Here we performed molecular docking calculations using the crystal structure of Leptomycin B (LMB)bound human CRM1 as a template to gain structural insight into the novel compounds' interactions with human CRM1 at an atomic level. Our results revealed that the binding of the compounds $\mathrm{C} 3$ and $\mathrm{C} 6$ is predicted to be centered around Cys528 which results in the blockage of 3 out of 5 hydrophobic pockets in which the NES peptides bind. Whereas, the compound C10 binding was predicted to be positioned below the reactive cysteine blocking 2 out of 5 hydrophobic pockets. In addition, the performed computational analysis identified several potential ligand binding sites outside the NES-binding cleft. Furthermore, docking calculations using different scoring functions and settings predicted the binding of DP2392-E10 at the top of HEAT9 loop, directly at the base of the acidic loop. Docking interactions of DP2392-E10 exhibited a non-covalent binding that involves several residues at the base of the acidic loop. The results of our study present new insight into CRM1 inhibition, in particular the allosteric mechanism mediated by the binding of DP2392-E10 outside the NES-binding cleft.

\subsection{Introduction}

CRM1 is an essential nucleocytoplasmic transport receptor that mediates the nuclear export of a wide range of proteins and ribonucleoprotein complexes [1], [2]. CRM1 mediated transport is dependent on the cooperative binding of a small GTPase Ran in its GTP bound form (RanGTP) and the cargo protein to the export receptor, forming a trimeric complex that transverse the nuclear pore complex. The overall architecture of CRM1 demonstrates a ring like structure that is composed of 21 HEAT repeats, each consisting of 2 antiparallel $\alpha$-helices $A$ and B connected via a short linker loop [3], [4]. CRM1 recognizes the cargo proteins by their leucin-rich nuclear export signal (NES) peptide. The NES peptide contains 4 to 5 hydrophobic residues that bind specifically 
in 5 hydrophobic pockets (Ф0- $\Phi 4$ ) located in the cleft between HEAT repeats $11 \mathrm{~A}$ and $12 \mathrm{~A}$ (referred to as the NES-binding cleft) [5], [6]. Overexpression of CRM1 was observed in several cancer diseases and it was identified as the major nuclear exporter for several oncoproteins, growth regulators, and suppressor protein like p53, p21, BRCA1/2, Rb, FOXO, which leads to cancer initiation and progression [7]-[10]. Furthermore, CRM1 plays a key role in several viral diseases as it is coopted by viruses like influenza, rabies virus $\mathrm{P}$, and HIV for the nuclear export of their RNA and ribonucleoprotein (RNP) complexes. This renders CRM1 an interesting drug target for therapeutic intervention in several cancer and viral diseases [11], [12].

Leptomycin B (LMB), a natural compound made by Streptomyces bacteria, was the first CRM1 inhibitor to be discovered [13], [14]. Its clinical tests revealed severe side effects and high toxicity [15], which induced a continuous search and development of alternative natural and synthetic compounds that could be used for CRM1 inhibition [11], [16]-[21]. A particular class of synthetic compounds known as selective inhibitors of nuclear export (SINE) were developed based on virtual screening using molecular modeling and simulations, physicochemical filters, and highthroughput molecular docking [22]. After several evaluations in pre-clinical and clinical studies, the SINE compound KPT330 (Selinexor) was the first CRM1-targeting compound to be approved for the treatment of relapsed or refractory multiple myeloma when taken in a combination with dexamethasone [23]. Structural analysis by means of X-ray crystallography in conjunction with computational analysis based on homology modeling and docking simulations defined the molecular basis of CRM1 inhibition. Furthermore, It has been used as a reliable approach for the development of novel CRM1 inhibitor [22], [24]. Earlier biochemical investigations revealed that LMB binding is dependent on a reactive cysteine residue (Cys528 in human) located at HEAT helix $11 \mathrm{~A}$ in the NES-binding cleft [25]. However, as the human protein failed to crystalize in complex with inhibitors, CMR1 from the yeast Saccharomyces cerevisiae ( $\left.{ }^{S c} \mathrm{CRM} 1\right)$ was genetically modified to incorporate the reactive cysteine (T539C) and was used to crystallize CRM1-LMB complex. The crystal structures of LMB bound to ${ }^{S C} \mathrm{CRM} 1$ revealed its covalent binding to $\mathrm{Cys} 528$ by Michael addition and defined its mechanism of action by blocking the 5 hydrophobic pockets in which the NES peptide binds [24]. Afterwards, the same approach was employed to solve the crystal structures of several KPT sine compounds and revealed their covalent junction to the reactive cysteine by the same mechanism as for LMB [26]-[29].

Recently we presented the crystal structure of LMB bound to human CRM1 $\left({ }^{H s} \mathrm{CRM} 1\right)$ in complex with RanGTP (Chapter 2). We furthermore investigated the molecular mechanism of the novel CRM1 inhibitors compounds C3, C6, and C10 [30] as well as the compound DP2392-E10 [31]. We 
tested their ability to interfere NES peptide-CRM1 binding and their dissociation of Snurportin1 (SPN1) export complex (CRM1-RanGTP-SPN1). In addition, we compared the compound interaction as well the binding of the NES peptide from protein kinase $A$ inhibitor (PKI $\Phi^{0}$ Leu NES) in ${ }^{H s} \mathrm{CRM} 1$ and ${ }^{S c} \mathrm{CRM} 1$. Our data showed that the compounds C3, C6, and C10 dissociate CRM1NES interactions by binding ${ }^{H s} \mathrm{CRM} 1$ at different rates in a Cys528 dependent manner (section 2.3.4). Whereas, the compound DP2392-E10 exhibited a unique binding behavior that is not dependent on the reactive cysteine (section 2.3.5). Furthermore, our recent study revealed that T539C variant of ${ }^{S C} \mathrm{CRM} 1$ is unable to bind the compounds $\mathrm{C} 3$ and $\mathrm{C} 6$, although their binding in human is Cys528 dependent (section 2.3.7). This proved that the crystallization approach using genetically modified ${ }^{S C} \mathrm{CRM} 1$ is limited and not applicable to all classes of CRM1 inhibitors, which further declare the importance of using human CRM1 for inhibitors' structural and biochemical characterization. Here we present our attempts to develop a crystallization approach for the structural characterization of ${ }^{H s} \mathrm{CRM} 1$-inhibitor complexes, by which we succeeded to solve the crystal structure of LMB bound to ${ }^{H s} \mathrm{CRM} 1$. We furthermore expanded our study by combining crystallographic and computational methods to gain structural insights into CRM1 inhibition by C3, C6, C10 and DP2392-E10. The performed crystallographic analysis unveiled an unexpected modification at the reactive cysteine during the crystallization trials of $\mathrm{C} 6$ in complex with human CRM1. Moreover, in silico docking calculations revealed the binding of DP2392-E10 outside the NES-binding cleft implicating a possible allosteric mechanism of CRM1 inhibition.

Our study provides a new path for the development of new classes of CRM1 inhibitors which might occupy different binding sites when compared with the LMB and SINEs compounds.

\subsection{Materials and Methods}

\subsubsection{Protein preparation and CRM1-inihibitor complexes crystallization}

HEAT 9 loop mutant ( ${ }^{430} V_{L V} 432$ to AAA) of the C-terminally truncated ( $\alpha$-helix; $\Delta 1037-1071$ ) ${ }^{H s} \mathrm{CRM} 1\left({ }^{H s} \mathrm{CRM}^{\Delta}{ }^{\Delta}\right)$ was expressed and purified as previously described (section 2.2.2). Human RanGTP1-180, Q69L [3] was prepared as described in [32]. For crystallization, ${ }^{H_{s}} \mathrm{CRM}{ }^{\Delta}{ }^{-}$-inhibitor complexes were prepared following the same protocol we used for preparing ${ }^{H s} \mathrm{CRM}{ }^{\Delta}{ }^{\Delta}$-RanGTPLMB complex (section 2.2.3) with the exception of adding C6 (synthesized by ChemBridge Corporation, USA) in 10 molar excess to CRM1 to counter its lower binding affinity in comparison

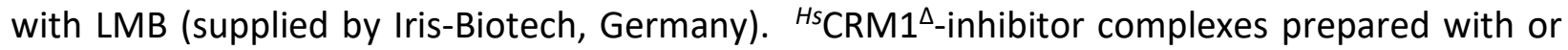
without the addition of RanGTP1-180, Q69L were used to setup crystallization trials at different concentrations using a wide range of sparse matrix screens. The screening was carried out using 
sitting drop vapor diffusion in 96-well 3-drop MRC crystallization plates (Molecular Dimensions) at $20^{\circ} \mathrm{C}$ or $4{ }^{\circ} \mathrm{C}$. Single diffracting crystals of the complexes ${ }^{H s} \mathrm{CRM} 1^{\Delta_{-}}$-RanGTP-LMB and ${ }^{H s} \mathrm{CRM} 1^{\Delta_{-}}$ RanGTP-C6 grew within 3-6 days in the commercial crystallization buffer Morpheus H10 [33] (10 \% w/v PEG 8000, 20 \% v/v ethylene glycol, 100 mM bicine /Trizma base pH 8.520 mM sodium Lglutamate, $20 \mathrm{mM}$ DL-alanine, $20 \mathrm{mM}$ glycine, $20 \mathrm{mM}$ DL-lysine $\mathrm{HCl}, 20 \mathrm{mM}$ DL-serine) when mixed in a ratio of 1:1 with the complex concentrated to $3 \mathrm{mg} / \mathrm{ml}$.

\subsection{2 ${ }^{\mathrm{Hs}} \mathrm{CRM} 1{ }^{\Delta}-$ RanGTP-C6 complex crystals dehydration and preparation}

Dehydration with PEG8000 was employed to improve the diffraction quality of ${ }^{H s} \mathrm{CRM} 1^{\Delta}$-RanGTPC6 complex crystals. Crystals were transferred to a $2 \mu \mathrm{L}$ crystallization buffer containing $20 \% \mathrm{w} / \mathrm{v}$ PEG8000. PEG8000 concentration was stepwise increased (5 \% steps) by slowly adding crystallization buffer containing $60 \%$ PEG8000. The drop was sealed and allowed to equilibrate for 45 min after each addition. Crystals treated with $25 \%$, $30 \%$, and $40 \%$ PEG8000 were flashed cooled in liquid nitrogen and used for X-ray diffraction experiment.

\subsubsection{Data collection, processing, and structure refinement}

X-ray diffraction data were collected at PETRA III EMBL beamline P13 (DESY, Hamburg, Germany), equipped with PILATUS 6M detector. A total of 35 datasets were collected from $28{ }^{H s} \mathrm{CRM}^{\Delta_{-}}$ RanGTP-C6 complex crystals including non-dehydrated and crystals treated with different concentrations of PEG8000. Data sets were indexed, processed and scaled using XDS package [34]. All datasets exhibited an orthorhombic lattice with the unit cell parameters $a=122.29 \AA, b$ $=151.33 \AA, c=234.84 \AA$ and belonging to the space group 1222. Structures were solved by means of molecular replacement using PHASER [35] with the crystal structure of the ${ }^{H s} \mathrm{CRM} 1^{\Delta}$-RanGTPLMB complex (PDB-ID 6TVO; hold until publication (HUPB)) (section 2.3.1) as the search model. Structures were refined to reasonable $R$ factors by iterative cycles of refinement and manual rebuilding in Refmac [36] and COOT [37] respectively. After refinement - in all datasets - a pronounced peak of positive electron density in mFo-dFC map was observed near the Cys528. The excess density was much smaller than expected for the compound $C 6$ and could not be explained by the compound. Further investigations by protein mass spectrometry analysis revealed the covalent modification of Cys528 by 2-Mercaptoethanol (BME) used during purification and crystallization as reducing agent. Subsequently, BME was modeled manually in COOT and its presence was verified using a Polder omit map [38]. Figures were generated with PyMol (The PyMOL Molecular Graphics System, Version 1.8 Schrödinger, LLC). 


\subsubsection{Identification of cysteine modification by liquid chromatography-mass spectrometry analysis}

Four samples were prepared for liquid chromatography-mass spectrometry (LC-MS) using ${ }^{H s} \mathrm{CRM} 1{ }^{\Delta},{ }^{H s} \mathrm{CRM} 1^{\Delta}$ mixed with crystallization buffer (Morpheus $\mathrm{H} 10$ ) and incubated overnight at $4{ }^{\circ} \mathrm{C}$ degree to mimic the crystallization environment, crystals grown using the complex ${ }^{H s} \mathrm{CRM} 1^{\Delta_{-}}$ RanGTP-C6, and crystals used for diffraction experiments. Cysteine reactive chemicals, reducing agents, and cysteine modifying steps were avoided throughout the entire procedure. Equal volumes of protein solutions were mixed with 2 X SDS sample buffer $(62.5 \mathrm{mM}$ Tris- $\mathrm{HCl}$ pH 6.8, $2.5 \%$ SDS, $0.002 \%$ bromophenol blue, $10 \%$ glycerol). Crystals not exposed to X-ray radiation were transferred and dissolved in a drop containing $1 X$ SDS sample buffer, while crystals used for diffraction experiments were carefully thawed in a drop of water and then were mixed in equal volume of $2 X$ SDS sample buffer. Afterwards, samples were boiled at $95^{\circ} \mathrm{C}$ for $5 \mathrm{~min}$ and applied for a brief separation in an SDS-PAGE gel. Protein-containing bands were cut and subjected to a trypsin (SERVA Electrophoresis, 37283.01) digestion according to Shevchenko et al [39]. Desalting of tryptic peptides prior to LC-MS was performed via StageTips according to the protocol described in [40]. $2 \mu \mathrm{L}$ of each sample were subjected to reverse phase liquid chromatography for peptide separation using an RSLCnano Ultimate 3000 system (Thermo Fisher Scientific). Peptides were loaded on an Acclaim PepMap 100 pre-column (100 $\mu \mathrm{m} \times 2 \mathrm{~cm}, \mathrm{C} 18,3 \mu \mathrm{m}, 100 \AA$; Thermo Fisher Scientific) with $0.07 \%$ trifluoroacetic acid at a flow rate of $20 \mu \mathrm{L} / \mathrm{min}$ for $3 \mathrm{~min}$. Analytical separation of peptides was done on an Acclaim PepMap RSLC column $(75 \mu \mathrm{m} \times 50 \mathrm{~cm}$, C18, $3 \mu \mathrm{m}, 100 \AA$; Thermo Fisher Scientific) at a flow rate of $300 \mathrm{~nL} / \mathrm{min}$. The solvent composition was gradually changed within 94 min from $96 \%$ solvent A ( $0.1 \%$ formic acid) and $4 \%$ solvent B ( $80 \%$ acetonitrile, $0.1 \%$ formic acid) to $10 \%$ solvent $B$ within 2 minutes, to $30 \%$ solvent $B$ within the next $58 \mathrm{~min}$, to $45 \%$ solvent B within the following $22 \mathrm{~min}$, and to $90 \%$ solvent B within the last $12 \mathrm{~min}$ of the gradient. All solvents and acids had Optima grade for LC-MS (Thermo Fisher Scientific). Eluting peptides were on-line ionized by nano-electrospray (nESI) using the Nanospray Flex Ion Source (Thermo Scientific) at $1.5 \mathrm{kV}$ (liquid junction) and transferred into a Q Exactive HF mass spectrometer (Thermo Fisher Scientific). Full scans in a mass range of 300 to $1650 \mathrm{~m} / \mathrm{z}$ were recorded at a resolution of 30,000 followed by data-dependent top $10 \mathrm{HCD}$ fragmentation at a resolution of 15,000 (dynamic exclusion enabled). LC-MS method programming and data acquisition was performed with the XCalibur 4.0 software (Thermo Fisher Scientific).

MS/MS2 data were searched against an E. coli-specific protein database (UniProt Proteome ID UP000000625) that additionally contained the CRM1 sequence using the software MaxQuant 
1.6.0.16 [41]. The digestion mode was trypsin/P, and the maximum number of missed cleavage sites was set to two. Oxidation at methionine and $\mathrm{N}$-terminal protein acetylation were set as variable modifications. A search for dependent peptides was performed to identify additional peptide modifications. The mass tolerances of precursors and fragment ions were $4.5 \mathrm{ppm}$ and 20 ppm (HCD), respectively. False discovery rates were calculated using the revert decoy mode, and the threshold for peptide sequence matches as well as protein identifications was 0.01 . MaxQuant output data were further evaluated using the Perseus software 1.6.0.7 [42]. The dependent peptide search provided evidence for the presence of a DeStreak (2mercaptoethanol, BME) modification ( $\triangle$ mass of 75.9983 ) at the cysteine residue of the CRM1 peptide DLLGLCEQK (D523-K531). Based on this result the data were searched against the same database as before using the Proteome Discoverer ${ }^{\mathrm{TM}}$ 2.2.0.388 software with the SequestHT search algorithm and the DeStreak modification at cysteines as a variable modification. Precursor mass tolerance and fragment mass tolerances were $10 \mathrm{ppm}$ and $0.02 \mathrm{Da}$, respectively. The digestion mode and false discovery rate were the same as for the MaxQuant analysis.

\subsubsection{Determination of potential ligand binding sites}

Potential ligand binding sites within human CRM1 were detected by using the Alpha Site Finder function of the MOE program (Chemical Computing Group Inc., Montreal, Canada) [43]. The score function of this program is used in combination with a geometry search method and based on generating hydrophobic or hydrophilic alpha spheres which serve as probes indicating for zones of tight atom packing [44]. Each determined binding pocket is defined by the number of contributing spheres, the number of hydrophilic points, residues at local surface and the number of sidechain contact atoms. For binding sites determination, human CRM1 from the complex structure ${ }^{H s} \mathrm{CRM}{ }^{1}{ }^{\Delta}$-RanGTP-LMB was used (PDB-ID 6TVO; HUPB). LMB and Ran GTP were removed from the model. RanGTP removal was important to avoid transiently formed pockets at the interaction surface between both proteins. For proper determination of the potential binding sites the missing Loop connecting the lower side of HEAT repeat helices 8B and 9A (Thr389Val401) was manually modeled in coot using the loop structure from the mouse CRM1 (PDB-ID $3 N B Z$, identical sequence in the modeled region) as a reference.

\subsubsection{In silico structure-based docking}

Molecular docking of DP2392-E10 within human CRM1 was performed using the Dock function of the MOE software suite (Chemical Computing Group Inc., Montreal, Canada). CRM1 structure prepared for potential ligand binding sites determination was used also for molecular docking. 
The model was further prepared in MOE software by adding hydrogen atoms, assigning partial charges, and removal of the non-polar hydrogens. The docking was performed against the entire CRM1 protein structure covering all determined potential binding sites. Docking simulations were performed using both the "Rigid Receptor" and "Induced Fit" docking protocols. The parameters used to calculate the score and interaction of the DP2392-E10 with CRM1 were selected as follows: Scoring function: London dG; Placement: Triangle matcher; Refinement: induced fit or rigid receptor; rescoring function: GBVI/WSA dG. The build in scoring function of MOE, S-score, was used to predict the highest binding affinity ( $\mathrm{kcal} / \mathrm{mol}$ ) of DP2392-E10 within the docking sites. The best binding pocket and docking orientation were selected based on the binding affinity score.

Site-specific molecular docking of the compounds C3, C6, and C10 within the NES-binding cleft of human CRM1 was conducted using AutoDock Vina [45]. Human CRM1 from the complex structure ${ }^{H s} \mathrm{CRM}^{\Delta}{ }^{\Delta}$-RanGTP-LMB was used (PDB-ID 6TVO; HUPB) upon removal of RanGTP and LMB. Receptor was prepared in AutoDockTools [46]; (hydrogen atoms were added, Gasteiger charges were computed, and non-polar hydrogens were then merged). Grid box accommodating the NES-binding cleft was defined in AutoDockTools with the center points: $X=-49.432, Y=-$ 28.086, $Z=-49.015$, and the size points: $X=28, Y=30, Z=22$. In order to gain a more realistic ligand-protein interaction environment, the side chains of the residues exposed to the potential binding site (Ile521, Leu525, Cys528, Glu529, Arg532, Lys534, Ala541, Ile544, Met545, Val548, Phe561, Thr564, Lys568, Leu569, Glu571, Phe572, Glu575, and Val580) were selected to be flexible during docking calculations. Furthermore, the flexibility of the ligands was applied at their torsional angles. After model and ligands preparation the docking calculations for all compounds were performed using AutoDock Vina. Each docking calculation resulted in 9 binding poses scored based on calculated binding free energy $(\mathrm{kcal} / \mathrm{mol})$. The best pose was selected based on the binding free energy value in combination with manual inspection of the binding orientation with respect to the Cys528.

\subsection{Results}

\subsubsection{Crystallization of human CRM1-inhibitor complexes}

In the last decade several natural and synthetic compounds were discovered or designed for the inhibition of CRM1 in order to develop anti-viral and anti-tumor drugs. Structural analysis by means of X-ray crystallography has been a powerful and a fundamental tool for understanding and optimizing protein-inhibitor interactions. Nevertheless, concerning CRM1 protein 
crystallization, the human export receptor has been the most challenging to study due to its high conformational flexibility [47]. In fact, out of 58 CRM1 structures deposited in the Protein Data Bank (PDB; https://www.rcsb.org/; [48]), only 5 structures are available for human protein (Figure 1). Two of these structure (PDB-IDs 4BSM and 4BSN) describe unliganded CRM1 lacking the Cterminal helical extension at resolutions of $4.5 \AA$ and $4.1 \AA$ [49] and one structure describes the C-terminal part (HEAT repeat helices 15A-21A) at a resolution of $2.3 \AA$ (PDB-ID 1W9C) [50]. On the other hand, there are 46 structures deposited in the PDB for CRM1 from the yeast Saccharomyces cerevisiae, in complexes representing different states of CRM1 export cycle or in complexes with inhibitors or NES peptides (Figure 1).

The first structure of ${ }^{S c} \mathrm{CRM} 1$ was solved in complex with RanGTP and RanBP1 (PDB-ID 3M1I) [51]. This complex structure in particular gained more interest due to the stability of the crystalized complex in the absence of a cargo protein, the well-defined crystallization conditions, reproducibility of well diffracting crystals, and more importantly because in this complex the NESbinding cleft is unliganded. Soon after, this approach was employed to crystalize ${ }^{5 c C R M 1-}$ inhibotor complexes by introducing the genetic mutation in ${ }^{5 C}$ CRM1 Thr539Cys (equivalent to Cys528 in ${ }^{H s}$ CRM1)-which allowed the binding of cysteine-dependent classical CRM1 inhibitors

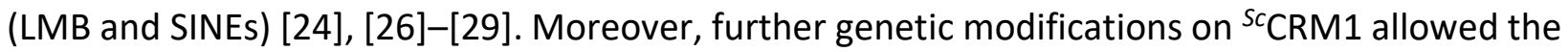
binding and the structural characterization of several NES peptides [6], [52]. This rendered the complex ${ }^{S C} C R M 1-R a n G T P-R a n B P 1$ a pragmatic model for characterizing CRM1 interactions with inhibitors and NES peptides. but on the other hand, it limited inhibitors characterization to the yeast ${ }^{S c} \mathrm{CRM} 1$. In our recent studies we compared the binding properties of human and yeast CRM1 towards several potential inhibitor as well as the NES from Protein Kinase A Inhibitor (PKI $\Phi^{\circ}$ Leu NES). Our data revealed that despite their overall structural similarity, the yeast CRM1 reveals a 16 folds less binding affinity than the human protein towards the NES peptide used. In addition, our data show that the T539C variant of yeast demonstrates very weak or no binding towards three of the characterized inhibitors, although their binding towards ${ }^{H s} \mathrm{CRM} 1$ is dependent on the reactive cysteine (section 2.3.7). Hence, these results prove that ${ }^{S c} C R M 1$ is not a universal model and is certainly limited to a specific class of inhibitors. Moreover, it further indicates for the importance of using ${ }^{H s} \mathrm{CRM} 1$ for studying inhibitors interactions. Therefore, our main goal was to obtain a crystallization approach that enables the structural characterization of human CRM1 inhibition. 


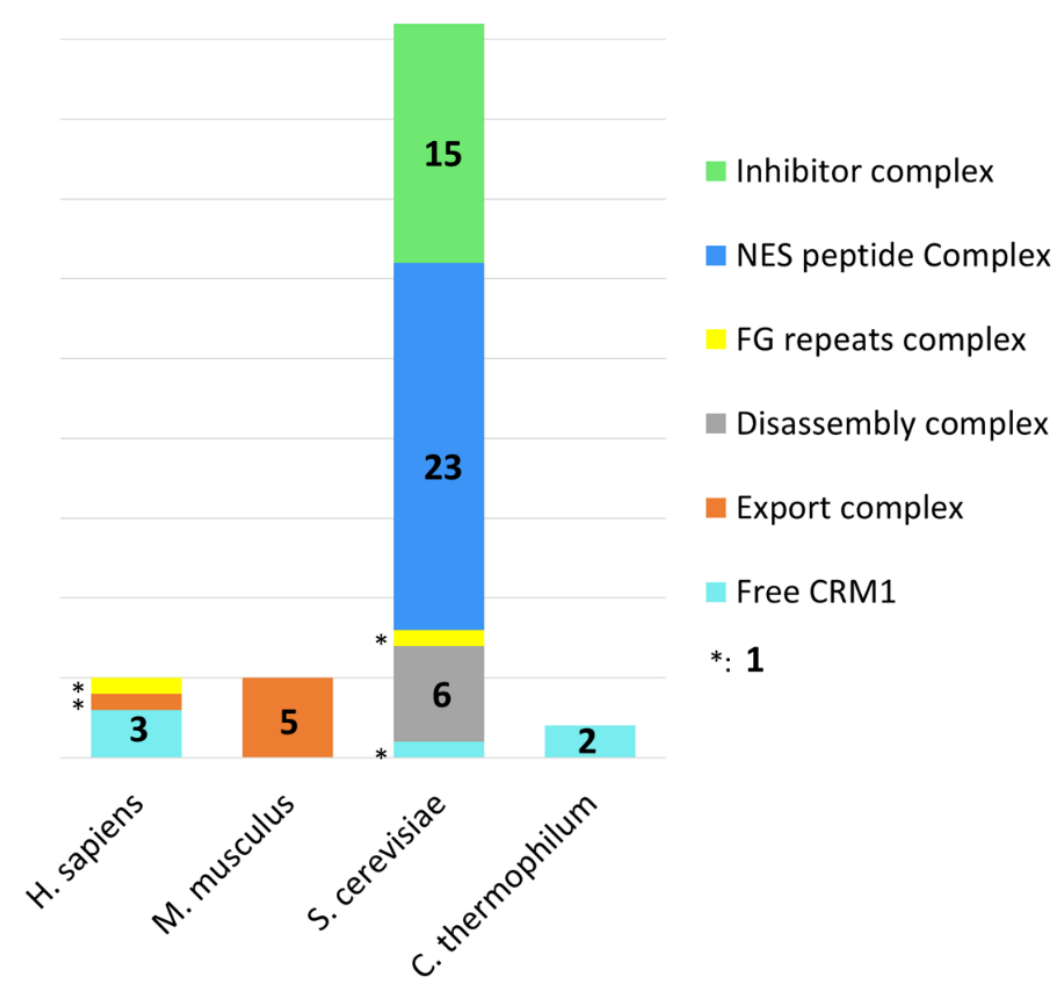

Figure 1: Numbers of CRM1 containing structures deposited in the Protein Data Bank and distributed by source organism. Different colors indicate for the sate represented by the crystal structure. Export complex: CRM1 in complex with Ran and/or cargo protein. Disassembly complex: CRM1 in complex with Ran and RanBP1 or RanBP2.

Initials crystallization screens of wild type ${ }^{H s}$ CRM1-inihibtor complexes failed to yield crystals in the presence and in the absence of RanGTP. Consequently, we constructed a more stabilized variant of ${ }^{H s} \mathrm{CRM} 1$ by truncating the $\mathrm{C}$-terminal helix ( $\left.\triangle 1037-1071\right)$ and mutating the acidic loop (HEAT9 loop) residues that interact with the back side of the NES-binding cleft ( ${ }^{430} \mathrm{VLV}^{432}$ to AAA). The effect of the introduced mutation on the conformational stability of ${ }^{H s} \mathrm{CRM} 1$ is described in detail in our study (section 2.3.1). In brief, the modified CRM1 variant $\left({ }^{H s} \mathrm{CRM} 1^{\Delta}\right)$ demonstrated a significantly increased NES binding affinity in the absence of RanGTP, indicating that the introduced mutations stabilize the protein towards the state in which the NES binding cleft is open and accessible by NES peptides or ligands (section 2.9 - supplementary figure 1). Crystallization screening of ${ }^{H s} C R M 1{ }^{\Delta}-L M B$ complexes in the presence or absence of RanGTP yielded crystals in several conditions. Few conditions containing variants of polyethylene glycol (PEG) or polyacrylic acid (PAA) as precipitants provided single crystals (Table 1). Relatively large single crystals of the complex ${ }^{H s} \mathrm{CRM} 1^{\Delta}-$ RanGTP-LMB grew within 3 days in the condition Morpheus $\mathrm{H} 10$ (Table 1) which contains a mixture of PEG8000 and ethylene glycol as a precipitant and several amino acids as additives. 

inhibition

Table 1: Crystallization conditions that yielded single crystals of ${ }^{H s} \mathrm{CRM} 1^{\Delta}-\mathrm{LMB}$ complexes in the presence and absence of RanGTP. PAA: polyacrylic acid. PEG: polyethylene glycol.

\begin{tabular}{|c|c|c|}
\hline Protein-inhibitor complex & Crystallization conditions & Observed crystals \\
\hline $\begin{array}{l}{ }^{H s} \text { CRM1 }{ }^{\triangle 1037-1071,430 V L V 432 ~ t o ~} \\
\text { AAA_LMB } \\
\text { Mixing ratio: } \\
\text { (1 mol : } 2 \text { mol) }\end{array}$ & $\begin{array}{l}\text { Reservoir solution: } 22 \% \text { PAA 5100, } 20 \\
\mathrm{mM} \mathrm{MgCl}_{2}, 100 \mathrm{mM} \text { HEPES pH } 6.5 \\
\text { Mixing ratio (protein: reservoir): } 2: 1 . \\
\text { Protein concentration: } 5 \mathrm{mg} / \mathrm{ml} \\
\text { Crystals growth time: } 17-24 \mathrm{~h} \\
\text { Incubation temperature: } 20^{\circ} \mathrm{C}\end{array}$ & \\
\hline $\begin{array}{l}{ }^{H s} \text { CRM1 }{ }^{\triangle 1037-1071,430 V L V 432 ~ t o ~} \\
\text { AAA_LMB } \\
\text { Mixing ratio: } \\
(1 \mathrm{~mol}: 2 \mathrm{~mol})\end{array}$ & $\begin{array}{l}\text { Reservoir solution: } 22 \% \mathrm{w} / \mathrm{v} \text { PAA } \\
5100,20 \mathrm{mM} \mathrm{MgCl}, 100 \mathrm{mM} \text { HEPES } \\
\mathrm{pH} 7.5,4 \% \text { 2,5-Hexanediol } \\
\text { Mixing ratio (protein: reservoir): 1:1. } \\
\text { Protein concentration: } 5 \mathrm{mg} / \mathrm{ml} \\
\text { Crystals growth time: } 1-3 \mathrm{~h} \\
\text { Incubation temperature: } 20^{\circ} \mathrm{C}\end{array}$ & \\
\hline $\begin{array}{l}{ }^{H s} \text { CRM1 }{ }^{\Delta 1037-1071,430 V L V 432 ~ t o ~} \\
\text { AAA }{ }^{H s} \text { RanGTP }{ }^{1-180,} \\
{ }^{\text {Q69L_LMB }} \\
\text { Mixing ratio: } \\
(1 \mathrm{~mol}: 1 \mathrm{~mol}: 2 \mathrm{~mol})\end{array}$ & $\begin{array}{l}\text { Reservoir solution: } 20 \% \mathrm{w} / \mathrm{v} \text { PEG- } \\
\text { monomethyl ether 2000, } 100 \mathrm{mM} \text { Tris } \\
\text { base } \mathrm{pH} 8.5,200 \mathrm{mM} \text { Trimethylamine } \\
\mathrm{N} \text {-oxide } \\
\text { Mixing ratio (protein: reservoir): } 1: 1 . \\
\text { Protein concentration: } 3 \mathrm{mg} / \mathrm{ml} \\
\text { Crystals growth time: } 7-10 \text { days } \\
\text { Incubation temperature: } 4^{\circ} \mathrm{C}\end{array}$ & $\begin{array}{l}\text { Blue color indicates } \\
\text { for UV absorption }\end{array}$ \\
\hline $\begin{array}{l}{ }^{H S} \text { CRM }^{1}{ }^{\Delta 1037-1071,430 V L V 432} \text { to } \\
\text { AAA }{ }^{H S} \text { RanGTP1-180, } \\
\text { Q69L_LMB } \\
\text { Mixing ratio: }(1 \mathrm{~mol}: 1 \mathrm{~mol} \\
: 2 \text { mol) }\end{array}$ & $\begin{array}{l}\text { Reservoir solution: } 10 \% \mathrm{w} / \mathrm{v} \text { PEG } \\
8000,20 \% \text { v/v ethylene glycol, } 100 \\
\mathrm{mM} \text { bicine /Trizma base } \mathrm{pH} 8.520 \\
\mathrm{mM} \text { sodium L-glutamate, } 20 \mathrm{mM} \mathrm{DL-} \\
\text { alanine, } 20 \mathrm{mM} \text { glycine, } 20 \mathrm{mM} \text { DL- } \\
\text { lysine } \mathrm{HCl}, 20 \mathrm{mM} \text { DL-serine. } \\
\text { (Morpheus H10) } \\
\text { Mixing ratio (protein: reservoir): } 1: 1 . \\
\text { Protein concentration: } 3 \mathrm{mg} / \mathrm{ml} \\
\text { Crystals growth time: } 3-6 \mathrm{days} \\
\text { Incubation temperature: } 4{ }^{\circ} \mathrm{C}\end{array}$ & \\
\hline
\end{tabular}


The observed crystals could not be produced using the wild type protein or in the absence of RanGTP. X-ray diffraction experiments using synchrotron radiation revealed that only crystals growing in this condition could diffract to a reasonable atomic resolution ranging from 3.2 to 4 $\AA$ A. In contrast, crystals grown in PAA-containing conditions could only diffract to a resolution of 8 $\AA ̊$ and crystals grown in other conditions did not show detectable diffraction pattern. Hence, our crystallization experiments were focused on the condition Morpheus $\mathrm{H} 10$.

With the obtained crystallization approach, we were able to solve the crystal structure ${ }^{H s} \mathrm{CRM} 1^{\Delta_{-}}$ RanGTP-LMB complex to a resolution of $3.2 \AA$ and get an insight into inhibitor interaction with human CRM1. Nevertheless, we were further interested to employ the same approach to gain structural knowledge about the compound C6. This compound exhibited a Cys528 dependent binding in ${ }^{H s} \mathrm{CRM} 1$ at a low micromolar range, while it did not bind show binding towards to yeast CRM1 variant T539C (sections 2.3.4, and 2.3.7). Following the same protocol were able to gain crystals that were very similar in appearance to LMB complex crystals (Figure 2A). To improve the diffraction quality of these crystals, they were dehydrated by stepwise increasing PEG8000 concentration. Non-dehydrated crystals could diffract to a resolution of $3.2 \AA$, while dehydrated crystals showed improved diffraction properties. Crystals treated with 30\% PEG8000 exhibited the most significantly improved diffraction pattern up to $2.7 \AA ̊$ resolution (Figure 2B).

A

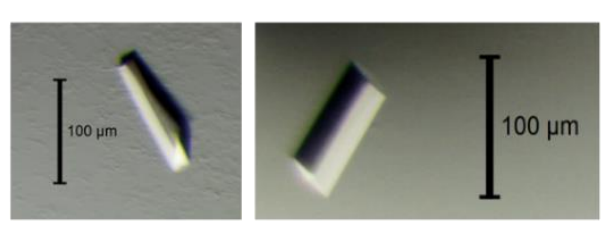

B

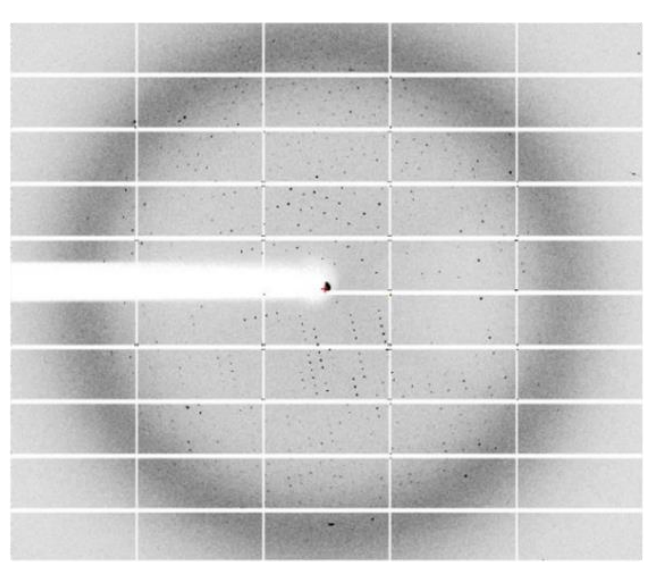

C

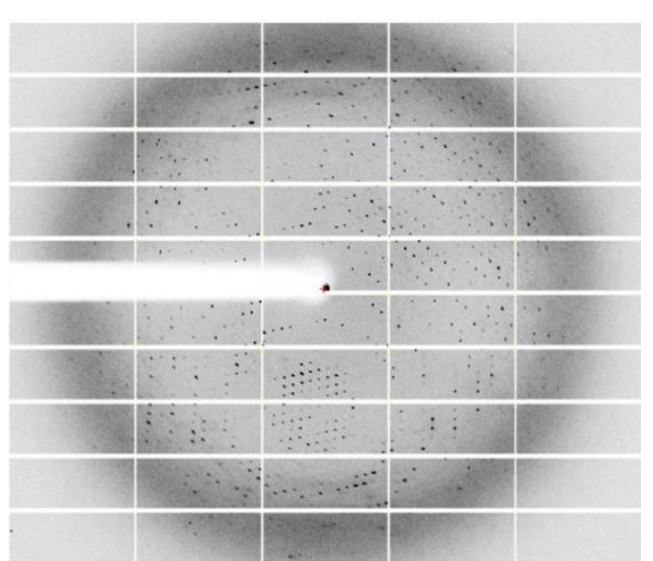

Figure 2: Optimization of crystals grown using the complex ${ }^{H s} \mathrm{CRM} 1^{\Delta}{ }_{-}{ }^{H s} \mathrm{RanGTP}-\mathrm{C} 6$ by dehydration. (A) Images of the obtained complex crystals. (B) A typical diffraction pattern of a non-dehydrated crystal. (C) a diffraction pattern of a crystal treated with 30\% PEG8000-containing crystallization buffer. 
Taken together, the genetic modifications introduced to human CRM1 enabled the protein to crystalize in a complex with RanGTP and the inhibitors in the crystallization condition Morpheus H10 (Table 1), which represents a crystallization strategy for studying inhibitors interactions with human CRM1. Nevertheless, the structures within the crystals grown using ${ }^{H s} \mathrm{CRM}{ }^{\Delta}{ }_{-}{ }_{H}{ }^{s} \mathrm{R}$ RanGTPC6 complex revealed an unexpected modification at the reactive cysteine residue (Cys528).

\subsubsection{Modification of Cys528 by 2-Mercaptoethanol}

Structure refinement of all datasets obtained from crystals grown using the complex ${ }^{H s} \mathrm{CRM} 1{ }^{\triangle}$ ${ }^{H}$ RanGTP-C6 displayed an excess difference electron density at the Cys528 (Figure 3A). The positive density in the mFo-DFc could not be explained by the compound $\mathrm{C} 6$.
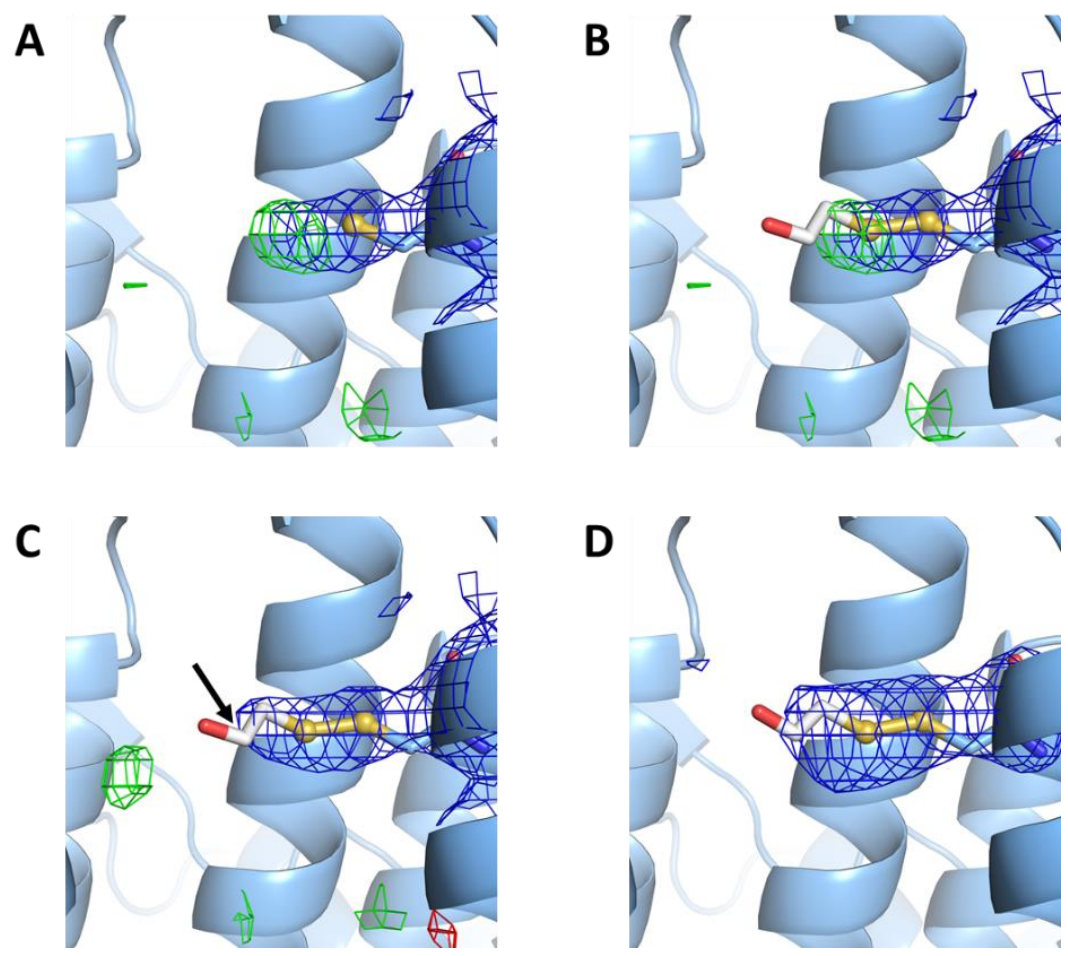

Figure 3: The modification of Cys528 by 2-mercaptoethanol (BME). Difference electron density maps $2 \mathrm{mFo}-\mathrm{dFc}$ (contoured at 1.0 sigma, in blue) and $\mathrm{mFo}-\mathrm{DFc}$ (contoured at 3.0 sigma in green and -3.0 sigma in red) around the Cys528, generated after model refinement with unmodified cysteine in (A and B) and after the refinement of covalently modified model -Cys528-BME conjugate- in (C). Non modified cysteine demonstrates an excess density representing a pronounced positive peak in the mFo-DFc map (A). The sulfur atom of the modeled BME occupies the center of the excess density observed around the Cys528 (B). (C) Crystallographic refinement of the atomic model comprising Cys528-MBE conjugate. Neither positive nor negative peaks of mFo-DFc map could be observed. The methyl hydroxy (indicated by arrow) group was not visible in the electron density map due to its free rotation. (D) A polder omit map of Cys528-BME conjugate (contoured at 6.0 sigma, in blue) confirms that Cys528 modification by BME explains the excess density. 
The center of the mFo-DFc electron density peak was located within $2 \AA$ from the sulfur atom of Cys528 indicating a possible covalently bound modification (Figure 3A). In order to confirm the type of modification and to elucidate whether it was introduced prior to crystallization or by synchrotron radiation, LC-MS analysis was performed for samples prepared from: purified ${ }^{H s}{ }^{H} \mathrm{CRM1}^{\Delta}{ }^{\Delta}{ }^{H s}{ }^{\mathrm{C} R M}{ }^{\Delta}{ }^{\Delta}$-RanGTP-C6 complex mixed with crystallization buffer (Morpheus H10), complex crystals not used for diffraction experiment, and from crystals exposed to synchrotron radiation.

A

\begin{tabular}{ccccccc}
\hline & $\mathrm{b}^{+}$ & $\mathrm{b}^{2+}$ & & $\mathrm{y}^{+}$ & $\mathrm{y}^{2+}$ & \\
Order & $(\mathrm{m} / \mathrm{z})$ & $(\mathrm{m} / \mathrm{z})$ & Sequence & $(\mathrm{m} / \mathrm{z})$ & $(\mathrm{m} / \mathrm{z})$ & Order
\end{tabular}

\begin{tabular}{lllllll}
1 & 116.03422 & 58.52075 & D & & & 9 \\
2 & 229.11828 & 115.06278 & L & 979.49510 & 490.25119 & 8 \\
3 & 342.20235 & 171.60481 & L & 866.41104 & 433.70916 & 7 \\
4 & 399.22381 & 200.11554 & G & 753.32697 & 377.16712 & 6 \\
5 & 512.30788 & 256.65758 & L & 696.30551 & 348.65639 & 5 \\
6 & 691.31534 & 346.16131 & C-DeStreak & 583.22144 & 292.11436 & 4 \\
7 & 820.35794 & 410.68261 & E & 404.21397 & 202.61063 & 3 \\
8 & 948.41651 & 474.71190 & Q & 275.17138 & 138.08933 & 2 \\
9 & & & K & 147.11280 & 74.06004 & 1 \\
\hline
\end{tabular}

B

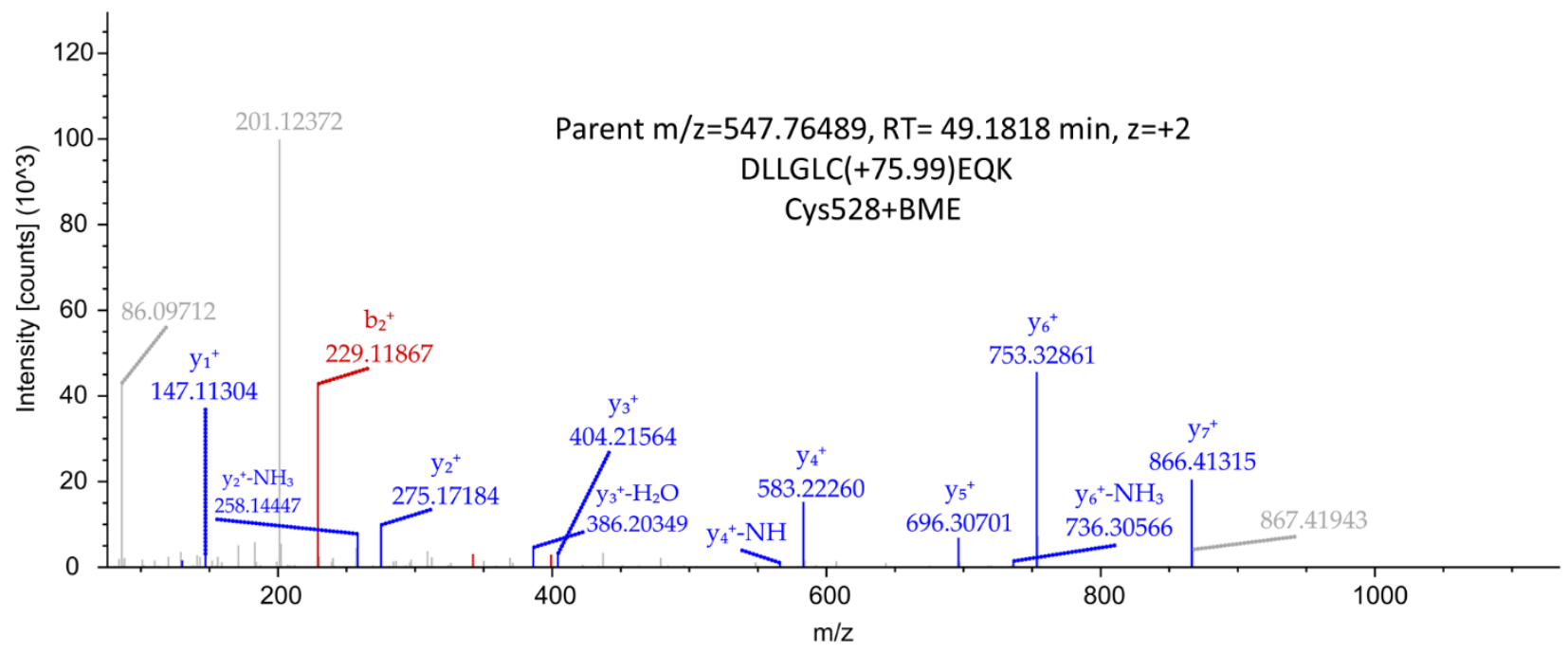

Figure 4: Determination of the Cys528 DeStreak modification of human CRM1 with 2mercaptoethanol (BME) using mass spectrometry analysis. (A) List of possible fragment ions of the Cys528-BME containing peptide (DLLGLC(+75.99)EQK) with detected y ions in blue and $b$ ions in red. (B) Representative fragmentation spectrum (1 of 15 ) of the respective precursor ion with $\mathrm{m} / \mathrm{z}$ of 547.765. RT, retention time. 
The results of the LC-MS analysis revealed a mass difference of 75.9983 at the cysteine residue within the tryptic peptide DLLGLCEQK (the reactive cysteine-Cys528). The observed shift corresponds to Cys-BME conjugate unveiling the covalent modification of Cys528 by BME, known as a DeStreak modification [53]. Although BME was introduced to CRM1 buffer during protein purification as a reducing agent, the covalent modification of Cys528 by BME was observed only when the purified protein was mixed with the crystallization buffer and in the complex crystals before and after exposure to synchrotron radiation. This further confirms that BME reactivity towards the cystine is induced by crystallization buffer conditions and that the observed density is not related to synchrotron radiation exposure.

Crystallographic refinement of the atomic model comprising Cys528-MBE conjugate explained the excess electron density (Figure 4B and C), which was also further supported by polder omit map as calculated with PHENIX (Figure 4D). While the disulfide bond fits electron density map, the freely rotatable methyl hydroxy group was not visible in the density what indicates its disorder. Nevertheless, the unexpected modification seems to interfere with the binding of the compound C6, which demonstrates that our crystallization approach needs to be further optimized either by screening for alternative crystallization buffer conditions or using a different reducing agent during proteins purification.

\subsubsection{Predicted binding conformations of the compounds $\mathrm{C} 3, \mathrm{C} 6$, and $\mathrm{C} 10$ in the NES- binding cleft}

Compounds C3, C6 and C10 (Figure 5A) were identified as efficient inducers of nuclear export inhibition. Preliminary studies demonstrated these compounds to cause the nuclear accumulation of HIV-1 Rev-NES when tested in different cancer cell lines [30]. Recently, we investigated the molecular mechanism of these 3 compounds by their ability to dissociate fluorescently labeled PKI $\Phi^{0}$ Leu NES binding to ${ }^{H s} \mathrm{CRM} 1$ (section 2.3.4). The results confirmed their inhibitory activity by concentration dependent binding to ${ }^{H s} \mathrm{CRM} 1$. The compound $\mathrm{C} 6$ exhibited the highest binding affinity $\left(I C_{50}=4.2 \mu \mathrm{M}\right)$ when compared to $\mathrm{C} 10\left(\mathrm{IC}_{50}=40 \mu \mathrm{M}\right)$ and $\mathrm{C} 3\left(\mathrm{IC}_{50}=59 \mu \mathrm{M}\right)$. Furthermore, the inhibitory activity of these compounds was strongly reduced upon changing cysteine to serine which confirmed their binding to be dependent on the reactive cysteine (Cys528) located in the NES-binding cleft of human CRM1 (section 2.3.4). In order to obtain structural information on these inhibitors' interactions with human CRM1, crystallization experiments in complex with ${ }^{H s} \mathrm{CRM}^{\Delta}{ }^{\Delta}$-RanGTP in the buffer condition Morpheus $\mathrm{H} 10$ were performed following the same approach employed to solve the crystal structure of ${ }^{H s} \mathrm{CRM}^{\Delta_{-}}$ 
RanGTP-LMB complex. Most crystallographic experiments were carried out using C6 as it demonstrated the highest binding affinity among the three compounds. However, the modification of Cys528 by BME (explained in the previous section) interfered with $\mathrm{C} 6$ binding and therefore structural information could not be obtained by the applied crystallographic approach. Alternatively, molecular docking - supported by the experimental findings - was performed to gain structural insights about possible binding orientations of these three compounds within the NES-binding cleft. Structure based - site specific docking was performed for each compound in the NES-binding cleft of human CRM1 model from the complex structure ${ }^{H s} \mathrm{CRM} 1{ }^{\Delta}$-RanGTP-LMB. Docking was performed with AutoDock Vina [45]. For more realistic docking environment residues with side chains exposed to the binding site were set as flexible during the simulation [46]. The best binding pose for each compound was selected based on the on the binding free energy value and the binding orientation with respect to Cys528.

The predicted binding of the compound C3 demonstrates an orientation centered around the Cys528 (Figure 5B); C3 is oriented with its tetrahydro-1-benzothiophene-3-carboxamide group pointing toward the top of the NES-binding cleft, whereas its 2-fluorobenzoyl group heads in the opposite direction toward the bottom of the NES-binding cleft. The hexafluoropropane-2,2diamine group of C3 binds deeply in the NES-binding cleft facing HEAT repeats $11 \mathrm{~A}$ and $12 \mathrm{~A}$ (Figure 5B). Similar to $\mathrm{C} 3$, the compound C6 docking exhibits an orientation that is also centered around the Cys528 (Figure 5A); while the chloro-(methylsulfonyl)benzene of C6 is pointing toward the top of the NES-binding cleft, the trimethoxybenzene group is oriented to the opposite direction pointing towards the bottom of the NES-binding cleft with 2 methoxy groups facing the NES-binding cleft and 1 methoxy group exposed to the solvent.The methyloxazol-amine group of the compound $\mathrm{C} 6$ is positioned in a close proximity of the reactive cysteine with the methylamine group exposed to the solvent (Figures $5 \mathrm{~B}$ and $5 \mathrm{C}$ ). Unlike the compounds $\mathrm{C} 3$ and $\mathrm{C6}$, the compound $\mathrm{C} 10$ is predicted to be bound in the middle of the NES-binding cleft, positioned below the reactive cysteine and sandwiched between HEAT repeats $11 \mathrm{~A}$ and $12 \mathrm{~A}$. C10 demonstrates a closer binding to HEAT repeat $12 \mathrm{~A}$ than $\mathrm{C} 3$ and $\mathrm{C} 6$, while its methoxy group pointing toward Cys528 (Figure 5B).

The docking binding of compounds C3 and C6 interfere with NES peptide binding by blocking the hydrophobic pockets $\Phi 2, \Phi 3$, and $\Phi 4$, while the binding of C10 blocks the pockets $\Phi 2$ and $Ф 3$ (Figures 5C and 5D). Furthermore, the predicted binding mode of C3 and C10 indicates they are mostly stabilized by hydrophobic interactions with CRM1 especially the hexafluoropropane-2,2diamine of C3 and trimethoxybenzene group of C6 since they bind deeply in the hydrophobic 
core of the NES-binding cleft. Nonetheless, their chemical groups pointing towards the upper part of the NES can also be stabilized by electrostatic interactions with surrounding lysins (Lys537 and Lys568). On the other hand, the predicted binding of C10 seems to be mediated exclusively by hydrophobic interactions as it binds in the regions forming the NES binding pockets $\Phi 2$ and Ф3 (Figures 5C and 5D). Yet, the flexible docking of the compound C3 exhibits different side chain conformation providing a wider binding pocket compared to that for C6 and C10 (Figure 5C) which allows the accommodation of the compound as it displays larger size than the other two.

A

Compound C3

Compound C6

Compound C10
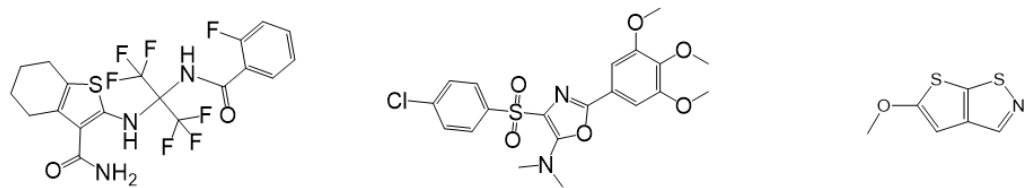

B
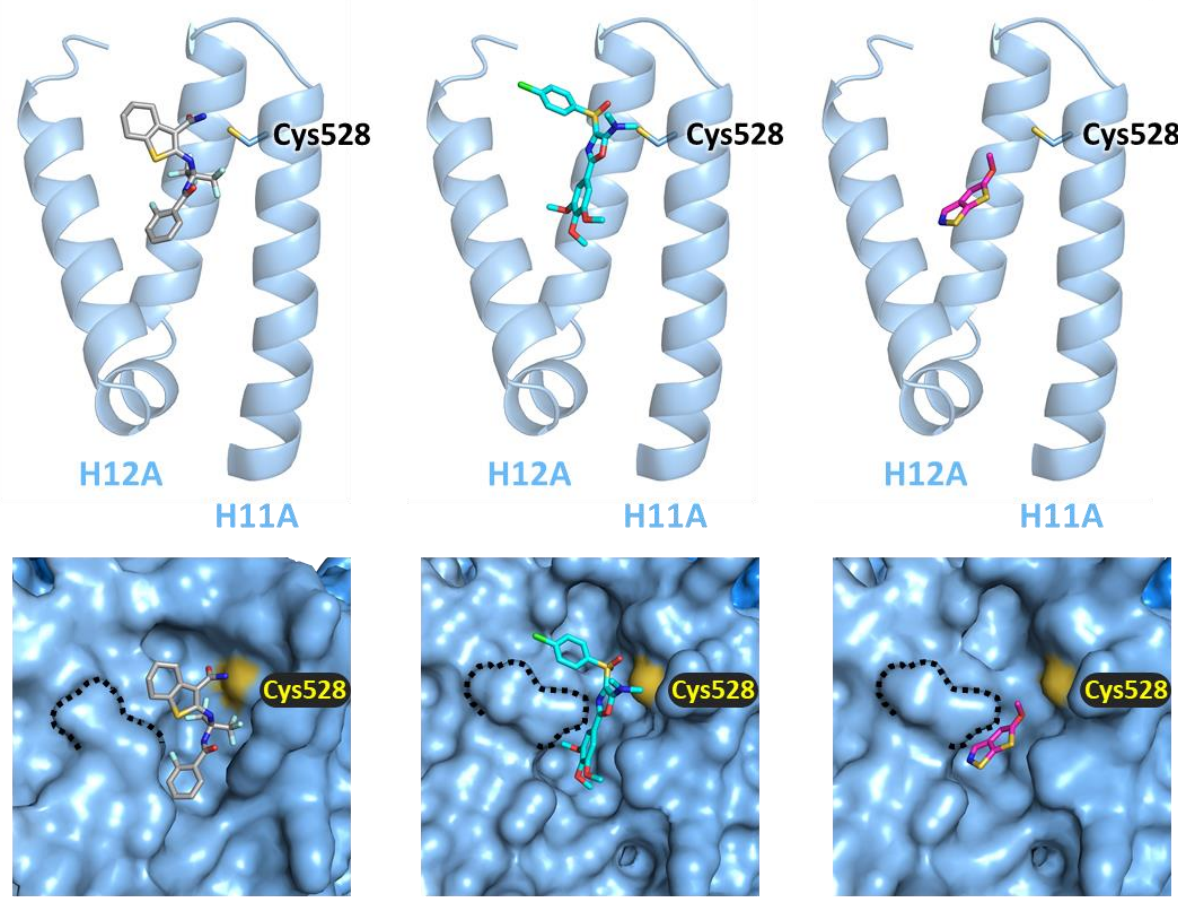

D

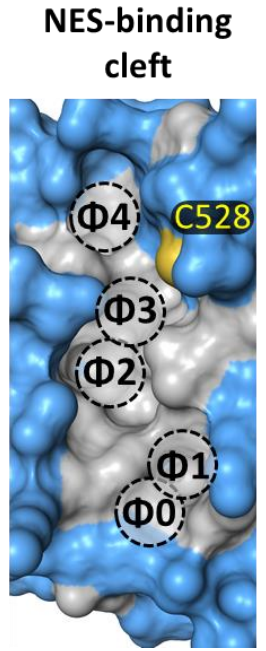

Figure 5: Predicted binding orientation of the compounds C3, C6, and C10 in the NES-binding cleft by molecular docking using AutoDock Vina. (A) chemical drawings of the compounds $\mathrm{C} 3$, $\mathrm{C} 6$, and C10. (B) The predicted binding conformations of C3, C6 and C10 (depicted as sticks) in the NESbinding cleft (cartoon representation). Cys528 which is involved in the binding of all three compounds is depicted as sticks. (C) surface representation of the putative binding pockets. Dashed lines indicate for the altered conformation in the side chains of C3 binding pocket residues compared to those in the binding pockets of C6 and C10. (D) Surface representation human CRM1 NES-binding cleft showing the respective $\Phi$ pockets (Ф0 - Ф4) that are involved in NES peptide binding. Hydrophobic regions are colored grey. 


\subsubsection{Human CRM1 exhibits multiple potential ligand binding sites outside the NES- binding cleft}

Several natural compounds (e.g. piperlongumine, goniothalamin, and anguinomycins) and synthetic compounds including KPT185, CBS9106, selinexor and verdinexor have been discovered or designed for the inhibition of CRM1. The inhibition of almost all these compounds was shown to be mediated via a the reactive cysteine (Cys528 in human CRM1) located in the NES-binding cleft [11], [20], [21], [54]-[56]. This indicated the binding of these compounds in the NES-binding cleft, which unveiled a common mechanism of action based on the inhibitors interfering with NES - CRM1 interaction by the direct blockage of the NES- binding cleft. The first possibility of inhibitors binding outside the NES has been reported based on the predicted binding of the compound DP2392-E10 near HEAT repeats 9 and 10 [57]. The binding of DP2392-E10 outside the NES-binding cleft was predicted by in silico study that involved molecular modelling and docking simulation. In that study a molecular model of human CRM1 was constructed by homology modelling using unliganded CRM1 from Chaetomium thermophilum (PDB ID 4FGV) as a template [57]. The constructed model was then recruited for the identification of potential binding sides using the site finder function in MOE software suite, which identified a total of 45 binding pockets within the prepared model. Here we followed the same approach for the identification of potential ligand binding sites, but alternatively using human CRM1 from the complex ${ }^{H s} \mathrm{CRM} 1{ }^{\Delta}$ -

${ }^{H}$ RanGTP-LMB crystal structure (PDB-ID 6TVO; HUPB), which provide an experimental model representing an inhibitor-bound human CRM1. A total of 47 potential ligand binding sites within the experimental model were defined by Site Finder module of MOE program.

The potential binding sites are mainly distributed at the outer surface of human CRM1 toroid, manly in the grooves between the HEAT repeat helices (Figure 6, side view). Several potential binding sites were also identified at the top of the HEAT repeats in the grooves formed between the linker loops of neighboring HEAT repeats (Figure 6, top view). In contrast, only few binding sites were identified at the lower side of the protein (Figure 6, bottom view). Few pockets formed at the interaction surfaces between the $\mathrm{N}$ and the $\mathrm{C}$ termini and between the acidic loop and the nearby HEAT repeats were also identified as potential binding sites (Figure 6, bottom view). However, these pockets are transiently present and only formed due to the stabilization of CRM1 in the conformation displayed by the crystal structure and therefore are less likely to be considerable binding sites. 


\section{Side view}

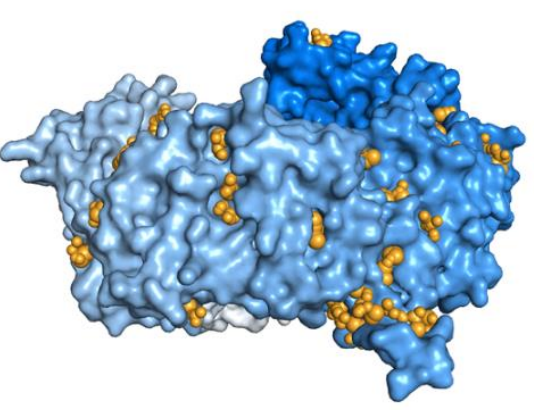

Top view

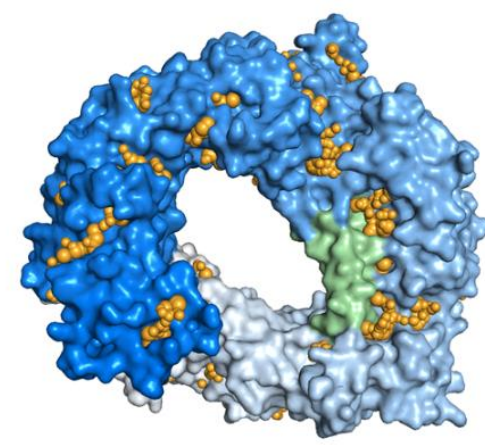

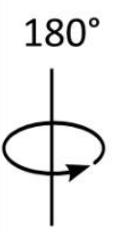

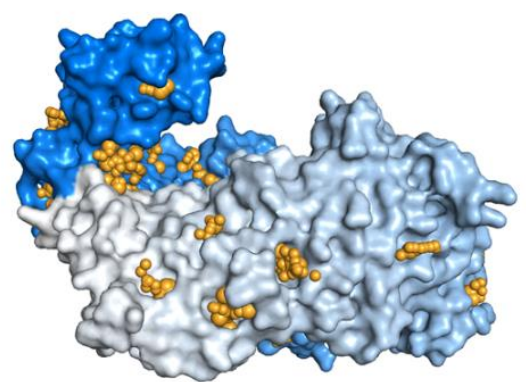

Bottom view

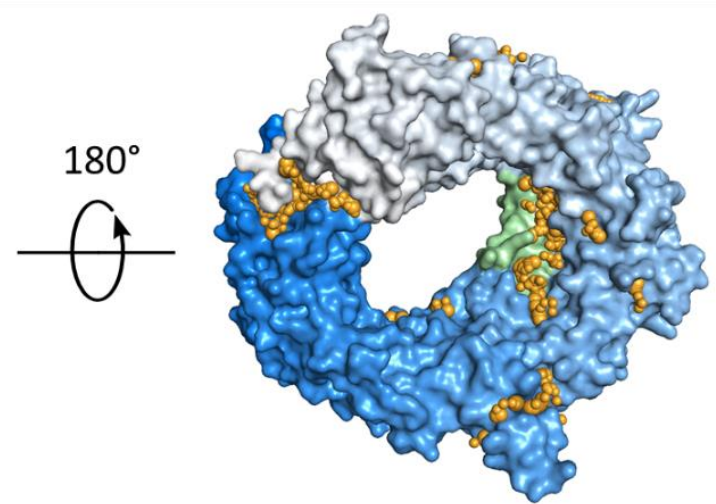

Figure 6: Potential binding sites of CRM1 inhibitors presented on the surface of human CRM1. Candidate binding sites (filled with orange spheres) were detected using the Alpha Site Finder function of the program MOE. CRM1 is depicted in surface representation and gradient colored from the $\mathrm{N}$-terminus (marine) to the $\mathrm{C}$-terminus (white). The acidic loop is shown in pale green.

\subsubsection{DP2392-E10 docking demonstrates unique binding interactions at the base of the} Acidic loop

DP2392-E10 demonstrated potentials for anti-viral drugs as it was shown to reduce the replication of influenza A virus by interfering with CRM1 mediated transport of viral nucleoprotein (VRNP) [31]. Further experiments revealed DP2392-E10 inhibitory activity by direct binding to CRM1. In silico docking predicted the binding of the compound outside the NESbinding cleft which suggested an alternative mechanism of action that is rather allosteric and thus different from the classical cysteine-dependent direct blockage of the NES- binding cleft [57]. The docking was based on homology model of human CRM1 as described in the previous section and in details by Chutiwitoonchai et al [57]. In our recent study we investigated DP2392E10 molecular mechanism of action by the dissociation of fluorescently labeled PKI $\Phi^{0}$ Leu NES CRM1 interaction as performed for the compounds C6, C3, and C10 using wild type and C528S 
mutant of ${ }^{H s}$ CRM1 (section 2.3.5). DP2392-E10 was able to dissociate NES binding to wild type ${ }^{H s} \mathrm{CRM} 1$ with an $\mathrm{IC}_{50}$ of $63 \mu \mathrm{M}$, in a similar range of $\mathrm{C} 3$ binding $\left(\mathrm{IC}_{50}=59 \mu \mathrm{M}\right)$. Unlike $\mathrm{C} 3, \mathrm{C} 6$ and C10 the inhibitory activity of DP2392-E10 was not reduced when cysteine was changed to serine which strongly support its allosteric binding outside the NES-binding cleft. Furthermore, our experiments showed that DP2392-E10 can dissociate SPN1 binding to CRM1, unlike C3 when added at the same concentration, which also indicated DP2392-E10 to have alternative mechanism of action to that of C3. In silico docking based on homology modeling by Chutiwitoonchai et al [57] provided a hint about DP2392-E10 binding to CRM1 summarized by its predicted binding near HEAT repeats 9 and 10 .

For better understanding of the novel binding mechanism of DP2392-E10 we aimed to obtain a detailed knowledge on its potential binding site and binding interactions by structure based in silico docking. Docking experiments were performed using human CRM1 model from the complex ${ }^{H s}$ CRM1- ${ }^{H s}$ RanGTP-LMB crystal structure (same model was used for potential binding side search). The missing loop connecting the helices 8B and 9A (Thr389-Val401) was constructed based on the loop structure of the exact sequence from mouse CRM1 (PDB-ID 3NBZ). The modeling of the loop was important for more accurate binding site identification and docking calculations due its critical location near HEAT repeats 9 and 10. Prior to docking calculations the model was prepared as described in materials and methods section. Docking was performed in the "rigid receptor" mode and the "induced fit" mode that allow for flexible interactions of the ligand with the docking site residues. Docking in the "rigid receptor" mode demonstrated the least binding energy for DP2392-E10 binding in a pocket located at the top of HEAT repeat 9 directly at the base of the acidic loop (HEAT9 loop) (Figure 7). The exact binding pocket and almost identical conformation exhibited the second lowest binding energy when docking was performed in the "induced fit" mode. Nevertheless, the pocket with the lowest binding energy score is a transiently formed pocket at the interaction surface between the acidic loop and nearby HEAT repeats and therefore was excluded as a true binding site. As a result, the most probable binding site of DP2392-E10 as suggested by rigid and induced fit docking is in is the pocket identified at the top of HEAT repeat 9, directly at the base of the acidic loop (Figure 7), which is also in alignment with previous prediction based on modeled ${ }^{H s} \mathrm{CRM} 1$. 


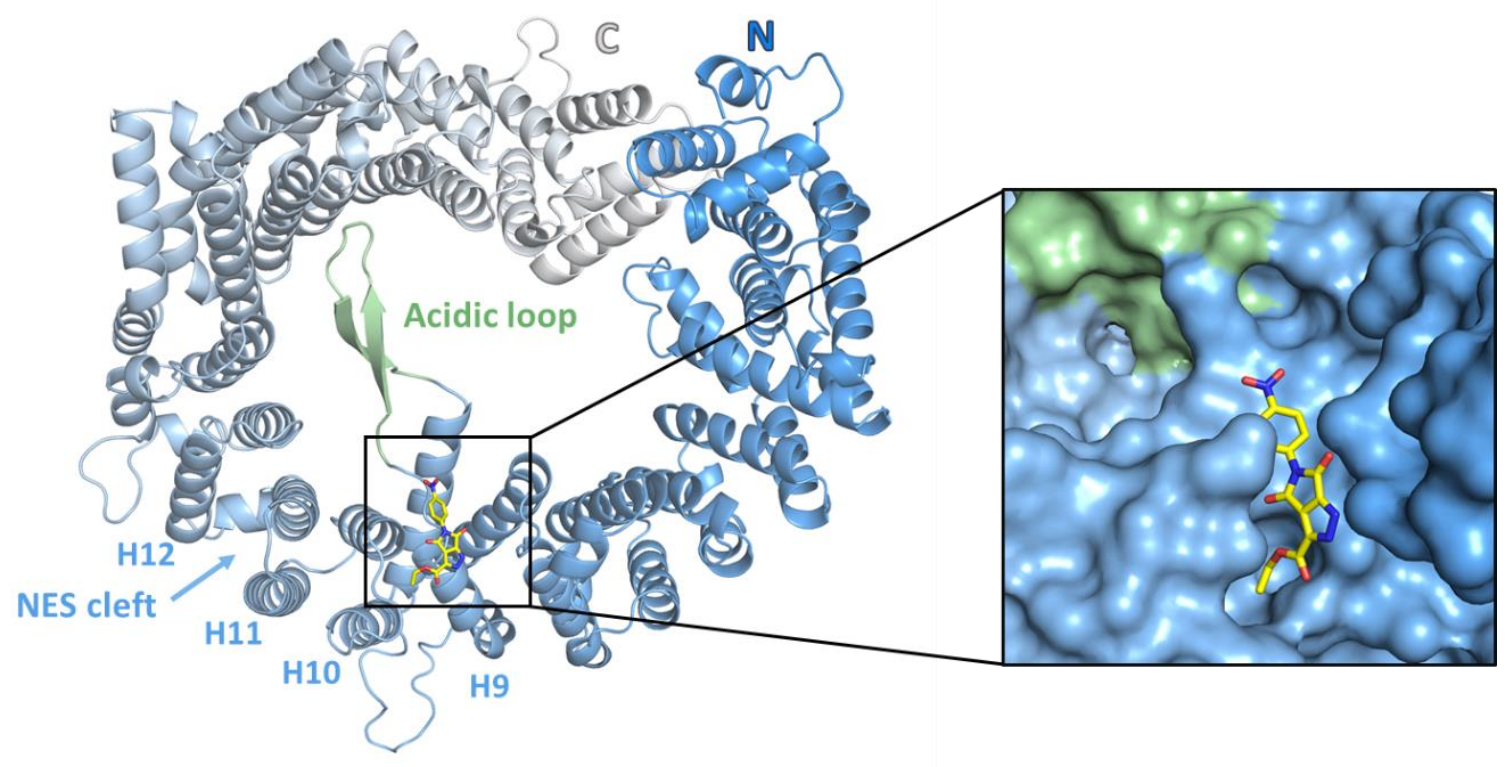

Figure 7: The predicted binding of DP2392-E10 at the base of the acidic loop between HEAT repeats 9 and 10 calculated by molecular docking in MOE software package. On the left, CRM1 is illustrated in cartoon representation and gradient colored from the $\mathrm{N}$-terminus (marine) to the C-terminus (white), while the acidic loop is shown in pale green. On the right, a zoomed in view of the binding site depicted in surface representation.

The docking pose shows the compound sandwiched between the first few residues of HEAT9 loop - that extend to form the acidic loop - and the residues of the upper part of HEAT repeat 10 (Figures 7 and 8A). DP2392-E10 is positioned at the top of HEAT repeat 9A with its nitrobenzene ring pointing towards the acidic loop, whereas the methoxyethane group at the opposite side is embedded between HEAT helices $10 \mathrm{~A}$ and 10B (Figure $8 \mathrm{~A}$ ). This predicted binding mode demonstrates a non-covalent network of mostly polar contacts and electrostatic interactions involving the residues Ser422, Arg423, and Lys426 at the base of HEAT9 loop, Lys479, Glu488, Gln483 of HEAT 10A, and Lys492, Asn493, and Thr496 of HEAT 10B (Figures 8A and 8B). Moreover, the predicted interaction network exhibit hydrophobic interactions with the residues Met424 and Ala425 at the base of HEAT9 loop, and the residue Trp499 of HEAT 10B (Figures 8A and $8 \mathrm{~B})$.

Altogether, the predicted binding interactions that involves several residues at the base of the acidic loop, supported by the biochemical experiment results, proposes a novel inhibitory mechanism of DP2392-E10 that is based on the allosteric interference with NES - CRM1 binding by modulating the conformation of the acidic loop. 
A

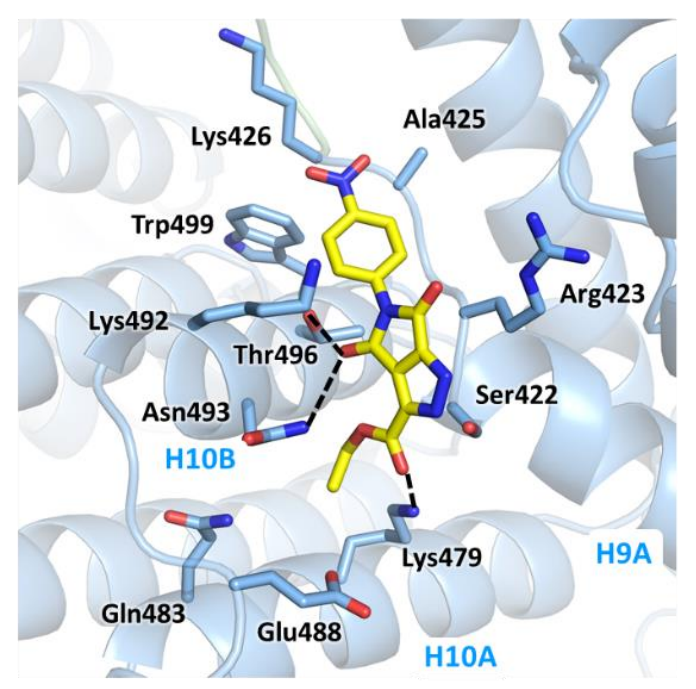

B

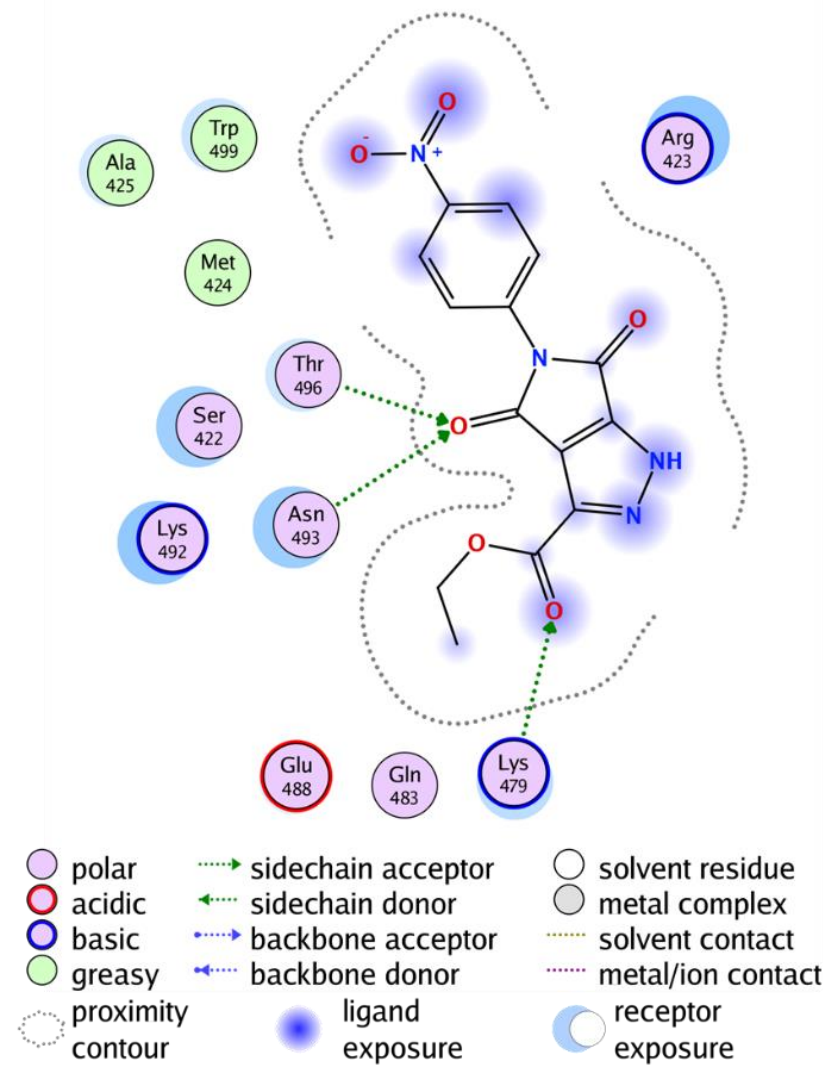

polar
acidic
basic
greasy
proximity
contour

(L45)

Figure 8: Molecular docking model of binding interactions of DP2392-E10 and human CRM1 generated by induced fit docking using MOE software. (A) A detailed view of the DP2392-E10 putative binding pocket (cartoon representation) showing the amino acids (depicted as sticks) that are involved in the inhibitor binding. The compound DP2392-E10 is shown as a stick model (carbon in yellow, oxygen in red and nitrogen in blue). Polar contacts are shown as black dashed lines. (B) 2D depiction of DP2392-E10 docking interactions with human CRM1 generated in MOE software.

\subsection{Discussion}

In our recent study we performed a biochemical characterization of the novel CRM1 inhibitions: compounds C3, C6, C10, and DP2392-E10. We also compared the interaction of these compounds towards human CRM1 and CRM1 from the yeast Saccharomyces cerevisiae that has been used as a model system for characterizing CRM1-inhibitors interaction over the last decade. Our results revealed the Cys528 dependent binding of the compounds C3 C6 and C10, despite the high variation in their chemical structure and composition (section 2.3.4). The compound DP2392E10, on the other hand, exhibited a unique interaction that is not dependent on the reactive 
cysteine residue which suggested it allosteric mechanism of action (section 2.3.5). In order to gain a better understanding of the molecular mechanism of the novel inhibitors we aimed to obtain structural information on C3, C6, and C10 binding conformation and on DP2392-E10 binding site and interactions. Since the yeast model was shown not to be valid for studying the compounds of interest (section 2.3.7) we focused on developing a crystallization approach that is based on human CRM1 - inhibitor complexes. Due to its high stability and strong binding affinity, LMB was used for crystallization screening of wild type - full length human CRM1 and several truncated and $\backslash$ or mutated variants. The intensive screening under different conditions succeeded to yield reasonably diffracting crystals when C-terminally truncated ( $\Delta 1037-1071)$ and acidic loop modified $\left({ }^{430} \mathrm{VLV}{ }^{432}\right.$ to AAA) ${ }^{H s} \mathrm{CRM} 1$ variant was used in combination with RanGTP. Using the obtained approach, we were able to gain structural insights into LMB - ${ }^{H s}$ CRM1 interactions (section 2.3.1). However, when the same method was applied using the compound C6, the crystal structure revealed the unexpected covalent modification of the Cys 528 by BME which became an obstacle towards obtaining structural information on C6 - CRM1 interactions (Figures 3 and 4). Although, BME was used as a reducing agent during CRM1 purification, mass spectrometry analysis of purified protein before and after mixing with crystallization buffer (Morpheus H10, Table 1) confirmed the modification to be induced by the buffer conditions. The reactivity of both the cysteine and BME is most probably induced due to the higher $\mathrm{pH}$ of crystallization buffer [58]. The utilized crystallization condition (Morpheus screen, $\mathrm{H} 10$ ) has a pH 8.5 maintained by a mixture of Bicine and Tris added to $100 \mathrm{mM}$, while the purification buffer has a $\mathrm{pH}$ of 7.8 maintained by $50 \mathrm{mM}$ HEPES (section 2.2.2). Changing the $\mathrm{pH}$ can induce the reactivity of the cysteine by the deprotonation of its thiol moiety (R-SH) when increased above its $\mathrm{p} K_{a}$ which is approximated for cysteine residues in proteins $\approx 8.5$ [59]. Furthermore, BME exhibit decreased stability as the $\mathrm{pH}$ increases which can lead to the formation of covalent adducts with surface cysteines [60]. Nevertheless, the covalent junction of Cys528 by BME was observed when $\mathrm{C} 6$ was used for crystallization but not when LMB was used, that was clearly defined by the electron density map of the crystal structure ${ }^{H s}$ CRM1 ${ }^{\Delta}$-RanGTP-LMB (Chapter 2 supplementary figure 2). This can be explained by the tight irreversible covalent binding of LMB to the Cys528. The incubation of CRM1 - inhibitor complexes prior to crystallization seems to be sufficient for $L M B$, as it binds in the $\mathrm{nM}$ range $\left(\mathrm{IC}_{50}=151 \mathrm{nM}\right)$, to irreversibly react with the cysteine, which results in a stable complex that prohibit the possible modification by BME upon subsequent mixing with the crystallization buffer. Whereas for $\mathrm{C} 6$, as it demonstrates weaker binding than $\operatorname{LMB}\left(\mathrm{IC}_{50}=4.2 \mu \mathrm{M}\right)$ and due to the possibility of its reversible binding, such a persistent stability could not be achieved. In summary, although with the obtained crystallization 
approach we were able to get a structural insight in human CRM1 -LMB interactions, the crystallization trials of C6 compound revealed that the applied approach exhibit some limitations due to the observed cysteine reactivity and therefore is still preliminary and requires further optimization. These limitations can be avoided by screening for alternative crystallization condition or the usage of more stable reducing agent during protein preparation like Tris(2carboxyethyl)phosphine (TCEP) that demonstrates a higher stability than BME at a wider $\mathrm{pH}$ range [61].

As crystallization of the ${ }^{H s} \mathrm{CRM} 1$ complexes with compounds $\mathrm{C} 3, \mathrm{C} 5$ and $\mathrm{C} 10$ demonstrated difficulties, molecular docking using human CRM1 from ${ }^{H s} \mathrm{CRM} 1{ }^{\Delta}$-RanGTP-LMB complex structure was applied for the prediction of their binding conformations. Docking calculations predicted a binding mode centered around the reactive cysteine for the compounds C3 and C6 (Figure 5B), which results in the blockade of 3 out of the 5 hydrophobic pockets in which the NES peptide specifically bind (Figures 5C and 5D). In contrast, the predicted binding of compound C10 was positioned below the reactive cysteine and revealed blocking of 2 out of the 5 hydrophobic pockets. Although both $\mathrm{C} 3$ and $\mathrm{C} 6$ mediates CRM1 inhibition by blocking the pockets $\Phi 2, \Phi 3$, and Ф4, the dissociation of NES binding by C3 exhibit 15 folds less binding affinity than C6 (section 2.3.4). Furthermore, in the same assay, $\mathrm{C} 10$ exhibited 5 folds higher binding affinity than $\mathrm{C} 3$ even though it is predicted to block only the hydrophobic pockets $\Phi 2, \Phi 3$. Our results suggest that although the binding of all three compound is mediated by Cys528, their binding affinity is still strongly influenced by their mechanism of binding (covalent or not covalent), and whether their binding is reversable or permanent, which in turn vary according to the differences in their chemical composition and structural flexibility (Figures 5A). Furthermore, the predicted binding of $\mathrm{C} 6$ and $\mathrm{C} 3$ in a very close proximity to Cys528 might indicate their binding mechanism by the covalent conjugation of the cysteine. However, most docking programs can only predict noncovalent interactions. Therefore, the possible covalent binding of the compounds $\mathrm{C} 3$ and $\mathrm{C} 6$ can be cleared by alternative approach like mass spectrometry and X-ray crystallography.

In this study we also focused on the characterization of the compound DP2392-E10, which was predicted by in silico docking to bind near HEAT repeats 9 and 10 [57]. The predicted binding of DP2392-E10 outside the NES-binding cleft triggered us to search for other possible potential binding sites. Using the site finder tool of MOE software 47 potential ligand binding sites were identified in human CRM1 and were distributed mainly at the outer surface and the top (Figure 6) of the protein from the N-terminus to the $\mathrm{C}$-terminus. The potential binding of CRM1 inhibitors at different regions of CRM1 indicates for multiple possible mechanisms by which inhibitors can 
interfere with NES peptide recognition or the formation of the ternary export complex. For example the binding at the around the $\mathrm{N}$-terminal region, especially helices forming the RanGTP binding domain (CRIME) [62], can interfere with Ran binding in prevent the formation of a stable export complex. Furthermore, the binding of compounds near regulatory regions like the Cterminal region or the acidic loop can interfere with NES binding by altering the opened and closed states of the NES - binding cleft. Taken together, the inhibition of CRM1 is not restricted on the direct blockage of the NES-binding cleft, which is the mechanism of all well studied inhibitors, and can be rather mediated through several possible mechanisms induced by binding outside the NES-biding cleft indicating a wide possibility for developing novel alternative drugs.

To gain structural insight into DP2392-E10 binding to human CRM1 we performed docking in the "rigid receptor" and "induced fit" modes in the docking function of MOE software. Docking experiment performed by Chutiwitoonchai et al [57] was based on a homology model of human CRM1 constructed using the crystal structure of unliganded CRM1 from Chaetomium thermophilum (PDB ID 4FGV) that represents CRM1 in the extended pitched conformation. Due to the high structural dynamic of CRM1, in this conformation the spacing between HEAT repeats is significantly reduced from that in the compact conformation - conformation stabilized in the liganded state - which change the dimensions of the identified binding pockets and consequently influence the minimal energy score during docking calculations. In addition, in this conformation the NES-binding cleft is significantly narrowed which may results in the false positive prediction of compounds binding outside the cleft [63]. Therefore, we performed our docking calculations using human CRM1 from ${ }^{H s} \mathrm{CRM} 1{ }^{\Delta}$-RanGTP-LMB complex crystal structure which, besides being an experimental model, represents an inhibitor-bound state of CRM1. The docking experiments identified the binding of DP2392-E10 at the top of HEAT repeat 9 where its directly located at the base of the acidic loop. The identified binding location is very critical as the acidic loop, together with the C-terminal helix, is considered to be a key regulator of the NES- binding cleft opening and closing mediated by overall conformational change of CRM1 [47], [63]. Furthermore, several structural studies demonstrated the acidic loop functions as an allosteric autoinhibitor that stabilizes CRM1 in a conformation that is unable to bind NES peptide in the absence of RanGTP [51]. This autoinhibitory effect is mediated by the "flipped back" position of the acidic loop in the absence of RanGTP where it interacts with the back of the NES-binding cleft specifically residues Val430, Leu431, and Val432 of acidic loop in human, which stabilized the NES-binding cleft in a closed conformation [51]. Upon the formation of the ternary export complex the binding of RanGTP induces a conformation change of the acidic loop leading to its detachment from the 
back of the NES cleft and consequently the opening of the cleft which makes it accessible for the NES peptide [62], [63]. The effect of the acidic loop on the NES opening and closing was further supported by our fluorescence polarization binding assay on CRM1 PKI $\Phi^{0}$ Leu NES interaction. Our data showed that introducing the acidic loop mutation ( ${ }^{430} \mathrm{VLV}^{432}$ to $\left.A A A\right)$ causes a significant increase in NES binding affinity in the absence of RanGTP (Chapter 2 - supplementary figure 1), which further demonstrates the importance of the acidic loop as a key regulator of CRM1-NES interactions. The detailed interactions of DP2392-E10 in the proposed binding site reveal tight interactions with the base of the acidic loop, mediated by polar contacts and electrostatic interactions with the residues Ser422, Arg423, and Lys426 and hydrophobic interactions residues Met424 and Ala425. The binding of DP2392-E10 at the base of the acidic loop can allosterically induce the closure of the NES-binding cleft by modulating the conformation of the acidic loop. This proposed allosteric mechanism of DP2392-E10 can explain its dissociation of SPN1 binding despite its relatively low binding affinity compared to LMB or C6 (section 2.3.5). It furthermore explains its independent binding on the Cys528 and its binding to the wild type CRM1 from Saccharomyces cerevisiae (Chapter 2-figure 5). This renders DP2392-E10 a unique CRM1 inhibitor with potentials for developing a novel CRM1 targeting drug with an allosteric mechanism of action different from the classical mechanism of LMB and the synthetic KPT compounds.

\subsection{Conclusion}

In this study we combined a crystallographic approach and computational methods to gain structural insights into human CRM1 inhibition by the compounds C3, C6, C10, and DP2392-E10. Here we described our attempts to develop a crystallization approach that involve human CRM1inihibtor complexes. Using C-terminally truncated ( $\Delta 1037-1071)$ and acidic loop modified $\left({ }^{430} \mathrm{VLV}^{432}\right.$ to $\left.\mathrm{AAA}\right){ }^{\mathrm{Hs}} \mathrm{CRM} 1$ variant in the complex with RanGTP we were able to solve the structure of $C R M 1^{\Delta}$-RanGTP-LMB complex (explained in detail in our recent study (Chapter 2 ). The obtained structure of inhibitor-liganded human CRM1 served as a docking model for the prediction of binding conformations of $\mathrm{C} 3, \mathrm{C} 6$ and $\mathrm{C} 10$, as well as the prediction of binding site and conformation of DP2392-E10. Site specific- flexible docking indicated the binding of C3 and C6 in the NES-binding cleft is centered around Cys528 which results in the blockage of the hydrophobic pockets $\Phi 2, \Phi 3$, and $\Phi 4$. Whereas the binding of $\mathrm{C} 10$ was predicted to be positioned below the reactive cysteine blocking the pockets $\Phi 2$ and $\Phi 3$. Furthermore, using the site finder function of MOE software, a total of 47 potential ligand binding site were identified in human CRM1 which were distributed mainly at the out surface of CRM1. Further investigation 
on DP2392-E10 identified its binding site located at the top of HEAT9 and directly at the base of the acidic loop. Docking experiments revealed that DP2392-E10 is likely to be stabilized by several electrostatic and hydrophobic interactions involving several residues at the base of the acidic loop, which together with the reported biochemical observations permit the conclusion that DP2392-E10 allosterically interferes NES binding by modulating the acidic loop conformation. Taken together, our structural analysis reveals a new aspect of CRM1 inhibition mediated by novel mechanism of action, which demonstrates strong potentials for the development of novel classes of anti-tumor and anti-viral drugs.

\subsection{References}

[1] T. Güttler and D. Görlich, "Ran-dependent nuclear export mediators: A structural perspective," EMBO Journal, vol. 30, no. 17. European Molecular Biology Organization, pp. 3457-3474, 31-Aug-2011.

[2] K. Kırlı et al., "A deep proteomics perspective on CRM1-mediated nuclear export and nucleocytoplasmic partitioning," Elife, vol. 4, no.

[3] T. Monecke, T. Güttler, P. Neumann, A. Dickmanns, D. Görlich, and R. Ficner, "Crystal structure of the nuclear export receptor CRM1 in complex with snurportin1 and RanGTP," Science (80-. )., vol. 324, no. 5930, pp. 1087-1091, May 2009.

[4] X. Dong et al., "Structural basis for leucine-rich nuclear export signal recognition by CRM1," Nature, vol. 458, no. 7242, pp. 1136-1141, Apr. 2009.

[5] T. La Cour, L. Kiemer, A. Mølgaard, R. Gupta, K. Skriver, and S. Brunak, "Analysis and prediction of leucine-rich nuclear export signals," Protein Eng. Des. Sel., vol. 17, no. 6, pp. 527-536, Jun. 2004.

[6] H. Y. J. Fung, S. C. Fu, and Y. M. Chook, "Nuclear export receptor CRM1 recognizes diverse conformations in nuclear export signals," Elife, vol. 6, pp. 1-13, 2017.

[7] R. Hill, B. Cautain, N. De Pedro, and W. Link, "Targeting nucleocytoplasmic transport in cancer therapy.," Oncotarget, vol. 5, no. 1, pp. 11-28, Jan. 2014.

[8] R. S. Faustino, T. J. Nelson, A. Terzic, and C. Perez-Terzic, "Nuclear transport: target for therapy.," Clin. Pharmacol. Ther., vol. 81, no. 6, pp. 880-6, Jun. 2007.

[9] J. G. Turner, J. Dawson, and D. M. Sullivan, "Nuclear export of proteins and drug resistance in cancer.," Biochem. Pharmacol., vol. 83, no. 8, pp. 1021-32, Apr. 2012.

[10] P. J. van der Watt and V. D. Leaner, "Regulation of Crm1 expression in cancer cells," 2010, pp. A33-A33. 
[11] C. Mathew and R. Ghildyal, "CRM1 inhibitors for antiviral therapy," Frontiers in Microbiology. 2017.

[12] A. Dickmanns, T. Monecke, and R. Ficner, Structural Basis of Targeting the Exportin CRM1 in Cancer, vol. 4, no. 3. 2015.

[13] T. Hamamoto, T. Uozumi, and T. Beppu, "Leptomycins A and B, new antifungal antibiotics III. Mode of action of leptomycin on schizosaccharomyces pombe," J. Antibiot. (Tokyo)., vol. 38, no. 11, pp. 1573-1580, Nov. 1985.

[14] N. Kudo et al., "Leptomycin B inhibition of signal-mediated nuclear export by direct binding to CRM1," Exp. Cell Res., vol. 242, no. 2, pp. 540-547, Aug. 1998.

[15] E. S. Newlands, G. J. Rustin, and M. H. Brampton, "Phase I trial of elactocin.," Br. J. Cancer, vol. 74, no. 4, pp. 648-9, Aug. 1996.

[16] S. Tamura, N. Shimizu, K. Fujiwara, M. Kaneko, T. Kimura, and N. Murakami, "Bioisostere of valtrate, anti-HIV principle by inhibition for nuclear export of Rev.," Bioorg. Med. Chem. Lett., vol. 20, no. 7, pp. 2159-62, Apr. 2010.

[17] T. R. Kau et al., "A chemical genetic screen identifies inhibitors of regulated nuclear export of a Forkhead transcription factor in PTEN-deficient tumor cells.," Cancer Cell, vol. 4, no. 6, pp. 463-76, Dec. 2003.

[18] X. Liu, Y. Chong, H. Liu, Y. Han, and M. Niu, "Novel reversible selective inhibitor of CRM1 for targeted therapy in ovarian cancer.," J. Ovarian Res., vol. 8, p. 35, Jun. 2015.

[19] D. Daelemans et al., "A synthetic HIV-1 Rev inhibitor interfering with the CRM1-mediated nuclear export.," Proc. Natl. Acad. Sci. U. S. A., vol. 99, no. 22, pp. 14440-5, Oct. 2002.

[20] S. Bonazzi et al., "Anguinomycins and Derivatives: Total Syntheses, Modeling, and Biological Evaluation of the Inhibition of Nucleocytoplasmic Transport," J. Am. Chem. Soc., vol. 132, no. 4, pp. 1432-1442, Feb. 2010.

[21] K. Sakakibara et al., "CBS9106 is a novel reversible oral CRM1 inhibitor with CRM1 degrading activity," Blood, vol. 118, no. 14, pp. 3922-3931, Oct. 2011.

[22] O. Kalid, D. Toledo Warshaviak, S. Shechter, W. Sherman, and S. Shacham, "Consensus Induced Fit Docking (cIFD): methodology, validation, and application to the discovery of novel Crm1 inhibitors.," J. Comput. Aided. Mol. Des., vol. 26, no. 11, pp. 1217-28, Nov. 2012.

[23] Y. Y. Syed, "Selinexor: First Global Approval," Drugs, 2019.

[24] Q. Sun et al., "Nuclear export inhibition through covalent conjugation and hydrolysis of Leptomycin B by CRM1," Proc. Natl. Acad. Sci., vol. 110, no. 4, pp. 1303-1308, 2013.

[25] N. Kudo et al., "Leptomycin B inactivates CRM1/exportin 1 by covalent modification at a cysteine residue in the central conserved region," Proc. Natl. Acad. Sci., 1999.

[26] R. Lapalombella et al., "Selective inhibitors of nuclear export show that CRM1/XPO1 is a 
target in chronic lymphocytic leukemia," Blood, vol. 120, no. 23, pp. 4621-4634, Nov. 2012.

[27] J. Etchin et al., "Antileukemic activity of nuclear export inhibitors that spare normal hematopoietic cells," Leukemia, 2013.

[28] J. D. Haines et al., "Nuclear export inhibitors avert progression in preclinical models of inflammatory demyelination," Nat. Neurosci., vol. 18, no. 4, pp. 511-520, Apr. 2015.

[29] Z. A. Hing et al., "Next-generation XPO1 inhibitor shows improved efficacy and in vivo tolerability in hematological malignancies," Leukemia, 2016.

[30] V. Fetz, S. K. Knauer, C. Bier, J. P. von Kries, and R. H. Stauber, "Translocation biosensors ? Cellular system integrators to dissect CRM1-dependent nuclear export by chemicogenomics," Sensors, vol. 9, no. 7, pp. 5423-5445, Jul. 2009.

[31] M. Kakisaka, T. Mano, and Y. Aida, "A high-throughput screening system targeting the nuclear export pathway via the third nuclear export signal of influenza $A$ virus nucleoprotein," Virus Research, vol. 217. Elsevier B.V., pp. 23-31, 02-Jun-2016.

[32] S. A. Port et al., "Structural and Functional Characterization of CRM1-Nup214 Interactions Reveals Multiple FG-Binding Sites Involved in Nuclear Export," Cell Rep., vol. 13, no. 4, pp. 690-702, 2015.

[33] F. Gorrec, "research papers The MORPHEUS protein crystallization screen research papers,"pp. 1035-1042, 2009.

[34] W. Kabsch, "Integration, scaling, space-group assignment and post-refinement," Acta Crystallogr. Sect. D Biol. Crystallogr., vol. 66, no. 2, pp. 133-144, 2010.

[35] A. J. McCoy, R. W. Grosse-Kunstleve, P. D. Adams, M. D. Winn, L. C. Storoni, and R. J. Read, "Phaser crystallographic software," J. Appl. Crystallogr., vol. 40, no. 4, pp. 658-674, Jul. 2007.

[36] M. D. Winn et al., "Overview of the CCP4 suite and current developments," Acta Crystallographica Section D: Biological Crystallography, vol. 67, no. 4. pp. 235-242, Apr2011.

[37] P. Emsley and K. Cowtan, "Coot: Model-building tools for molecular graphics," Acta Crystallogr. Sect. D Biol. Crystallogr., vol. 60, no. 12 I, pp. 2126-2132, Dec. 2004.

[38] D. Liebschner et al., "Polder maps: improving OMIT maps by excluding bulk solvent.," Acta Crystallogr. Sect. D, Struct. Biol., vol. 73, no. Pt 2, pp. 148-157, 2017.

[39] A. Shevchenko, M. Wilm, O. Vorm, and M. Mann, "Mass spectrometric sequencing of proteins from silver-stained polyacrylamide gels," Anal. Chem., vol. 68, no. 5, pp. 850858, 1996.

[40] J. Rappsilber, M. Mann, and Y. Ishihama, "Protocol for micro-purification, enrichment, pre-fractionation and storage of peptides for proteomics using StageTips," Nat. Protoc., 
vol. 2, no. 8, pp. 1896-1906, Aug. 2007.

[41] J. Cox and M. Mann, "MaxQuant enables high peptide identification rates, individualized p.p.b.-range mass accuracies and proteome-wide protein quantification," Nat. Biotechnol., vol. 26, no. 12, pp. 1367-1372, Dec. 2008.

[42] S. Tyanova et al., "The Perseus computational platform for comprehensive analysis of (prote)omics data," Nature Methods, vol. 13, no. 9. pp. 731-740, 30-Aug-2016.

[43] "Molecular Operating Environment (MOE) | MOEsaic | PSILO." [Online]. Available: https://www.chemcomp.com/Products.htm. [Accessed: 20-Jan-2020].

[44] K. Tøndel, E. Anderssen, and F. Drabløs, "Protein Alpha Shape (PAS) Dock: A new gaussian-based score function suitable for docking in homology modelled protein structures," J. Comput. Aided. Mol. Des., vol. 20, no. 3, pp. 131-144, Mar. 2006.

[45] O. Trott and A. J. Olson, "Software news and update AutoDock Vina: Improving the speed and accuracy of docking with a new scoring function, efficient optimization, and multithreading," J. Comput. Chem., vol. 31, no. 2, pp. 455-461, Jan. 2010.

[46] G. M. Morris et al., "Software news and updates AutoDock4 and AutoDockTools4: Automated docking with selective receptor flexibility," J. Comput. Chem., vol. 30, no. 16, pp. 2785-2791, Dec. 2009.

[47] N. Dölker et al., "Structural determinants and mechanism of mammalian CRM1 allostery," Structure, vol. 21, no. 8, pp. 1350-1360, Aug. 2013.

[48] H. M. Berman et al., "The Protein Data Bank," 2000.

[49] C. Dian et al., "Structure of a truncation mutant of the nuclear export factor CRM1 provides insights into the auto-inhibitory role of its C-terminal helix," Structure, vol. 21, no. 8, pp. 1338-1349, Aug. 2013.

[50] C. Petosa et al., "Architecture of CRM1/Exportin1 suggests how cooperativity is achieved during formation of a nuclear export complex," Mol. Cell, vol. 16, no. 5, pp. 761-775, Dec. 2004.

[51] M. Koyama and Y. Matsuura, "An allosteric mechanism to displace nuclear export cargo from CRM1 and RanGTP by RanBP1," EMBO J., 2010.

[52] H. Y. J. Fung, S. C. Fu, C. A. Brautigam, and Y. M. Chook, "Structural determinants of nuclear export signal orientation in binding to exportin CRM1," Elife, vol. 4, no. September 2015, pp. 1-19, 2015.

[53] H. J. Kim, S. Ha, H. Y. Lee, and K. J. Lee, "Rosics: Chemistry and proteomics of cysteine modifications in redox biology," Mass Spectrom. Rev., vol. 34, no. 2, pp. 184-208, Mar. 2015.

[54] M. Niu et al., "Piperlongumine is a novel nuclear export inhibitor with potent anticancer activity.," Chem. Biol. Interact., vol. 237, pp. 66-72, Jul. 2015. 
[55] J. Y. Wach, S. Güttinger, U. Kutay, and K. Gademann, "The cytotoxic styryl lactone goniothalamin is an inhibitor of nucleocytoplasmic transport," Bioorganic Med. Chem. Lett., vol. 20, no. 9, pp. 2843-2846, May 2010.

[56] J. Etchin et al., "KPT-330 inhibitor of CRM1 (XPO1)-mediated nuclear export has selective anti-leukaemic activity in preclinical models of T-cell acute lymphoblastic leukaemia and acute myeloid leukaemia," Br. J. Haematol., vol. 161, no. 1, pp. 117-127, Apr. 2013.

[57] N. Chutiwitoonchai et al., "Inhibition of CRM1-mediated nuclear export of influenza A nucleoprotein and nuclear export protein as a novel target for antiviral drug development," Virology, vol. 507, no. April, pp. 32-39, Jul. 2017.

[58] L. B. Poole, "The basics of thiols and cysteines in redox biology and chemistry," Free Radical Biology and Medicine, vol. 80. pp. 148-157, 2015.

[59] J. Kyte, "Structure in Protein Chemistry," Nov. 2006.

[60] P. T. Wingfield, "Use of Protein Folding Reagents," Curr. Protoc. Protein Sci., vol. 00, no. 1, p. Appendix, Jun. 1995.

[61] E. B. Getz, M. Xiao, T. Chakrabarty, R. Cooke, and P. R. Selvin, "A comparison between the sulfhydryl reductants tris(2-carboxyethyl)phosphine and dithiothreitol for use in protein biochemistry," Anal. Biochem., vol. 273, no. 1, pp. 73-80, Aug. 1999.

[62] T. Monecke, A. Dickmanns, and R. Ficner, "Allosteric control of the exportin CRM1 unraveled by crystal structure analysis," FEBS J., vol. 281, no. 18, pp. 4179-4194, Sep. 2014.

[63] T. Monecke et al., "Structural basis for cooperativity of CRM1 export complex formation.," Proc. Natl. Acad. Sci. U. S. A., vol. 110, no. 3, pp. 960-5, Jan. 2013. 


\section{Chapter 4: Discussion}

Structural characterization of CRM1-inhibitor complexes has been previously performed using CRM1 from the yeast Saccharomyces cerevisiae that was genetically modified to bind classical CRM1 inhibitors (Chapter 1 - table 1). This yeast CRM1 variant has been used as a model system for studying the exportin interaction with several natural and synthetic inhibitors, which has led to the definition of a typical mechanism of CRM1 inhibition requiring the covalent modification of the reactive cysteine residue that is located in the NES-binding cleft (J. Etchin et al. 2013; Haines et al. 2015; Hing et al. 2016; Kalid et al. 2012; Lapalombella et al. 2012; Sun et al. 2013). The overall aim of this study was to obtain structural and biochemical insights into human CRM1 inhibition. This work focused on the biochemical characterization and structural analysis of four novel nuclear export inhibitor candidates; the compounds: C3, C6, C10, and DP2392-E10 (Chutiwitoonchai et al. 2017; Fetz et al. 2009). In addition, LMB was engaged in this study for the development of a crystallization approach that is feasible for human CRM1. The crystal structure of ${ }^{H s} \mathrm{CRM1}{ }^{H s}$ RanGTP-LMB reported in this study together with several binding assays (involving inhibitor and NES peptide) revealed significant differences between human and yeast CRM1 in terms of binding affinities and binding interactions at the atomic level. Furthermore, integrative experimental and computational analysis of novel inhibitors showed that the compound C3, C6, and C10 dissociate CRM1-NES interaction by binding CRM1 through the reactive cysteine (Cys528). Docking calculations indicated similar binding modes of C3 and C6 that are centered around Cys528 and are different from the binding of $\mathrm{C} 10$ in the NES-binding cleft. In contrast, DP2392-E10 exhibited unique binding properties that lead to the dissociation of CRM1- NES interactions independent of the reactive cysteine. Docking simulations predicted the binding of DP2392-E10 outside the NES-binding cleft, at the base of the acidic loop, suggesting a novel mechanism of CRM1 inhibition by the allosteric modulation of the NES-binding cleft. Furthermore, computational analysis of inhibitor-bound human CRM1 identified several potential ligand binding sites outside the NES-binding cleft. This implicates several possible mechanisms of inhibiting the exportin.

While most aspects concerning these results were already discussed in detail in chapters 2 and 3 , the overall outcome of both sections will be discussed here in a broader perspective, focusing mainly on alternative mechanisms of CRM1 inhibition and potential structure-based methods for CRM1-targeting drug design. 


\subsection{High conformational stability is a major determinant of human CRM1 crystallizability}

Screening for crystallization conditions of full length-wild type human CRM1 failed to yield crystals in the presence or the absence of RanGTP. A major factor that obstacles human CRM1 crystallization is its high conformational flexibility, as in solution it samples between two extreme conformations, an extended and a compact one (Dölker et al. 2013; Monecke et al. 2013). In comparison to yeast CRM1, human CRM1 demonstrates a remarkably higher conformational flexibility that is mainly modulated by key structural elements including the C-terminal helix and the acidic loop (Dölker et al. 2013) (section 1.2.3). Truncating the C-terminal helix ( $\Delta 1037-1071)$ and modifying the acidic loop ( ${ }^{430} \mathrm{VLV}^{432}$ to $\left.\mathrm{AAA}\right)$ resulted in shifting the CRM1 conformational equilibrium towards the compact conformation which is characterized by an open and accessible NES-binding cleft (section 2.3.1). This was indicated by the pronounced increase of PKI $\Phi^{0}$ Leu NES binding affinity in the absence of RanGTP towards the modified human CRM1 $\left({ }^{H s} \mathrm{CRM} 1^{\Delta}\right)$ compared to the wild type protein (Chapter 2 - supplementary figure 1 ).

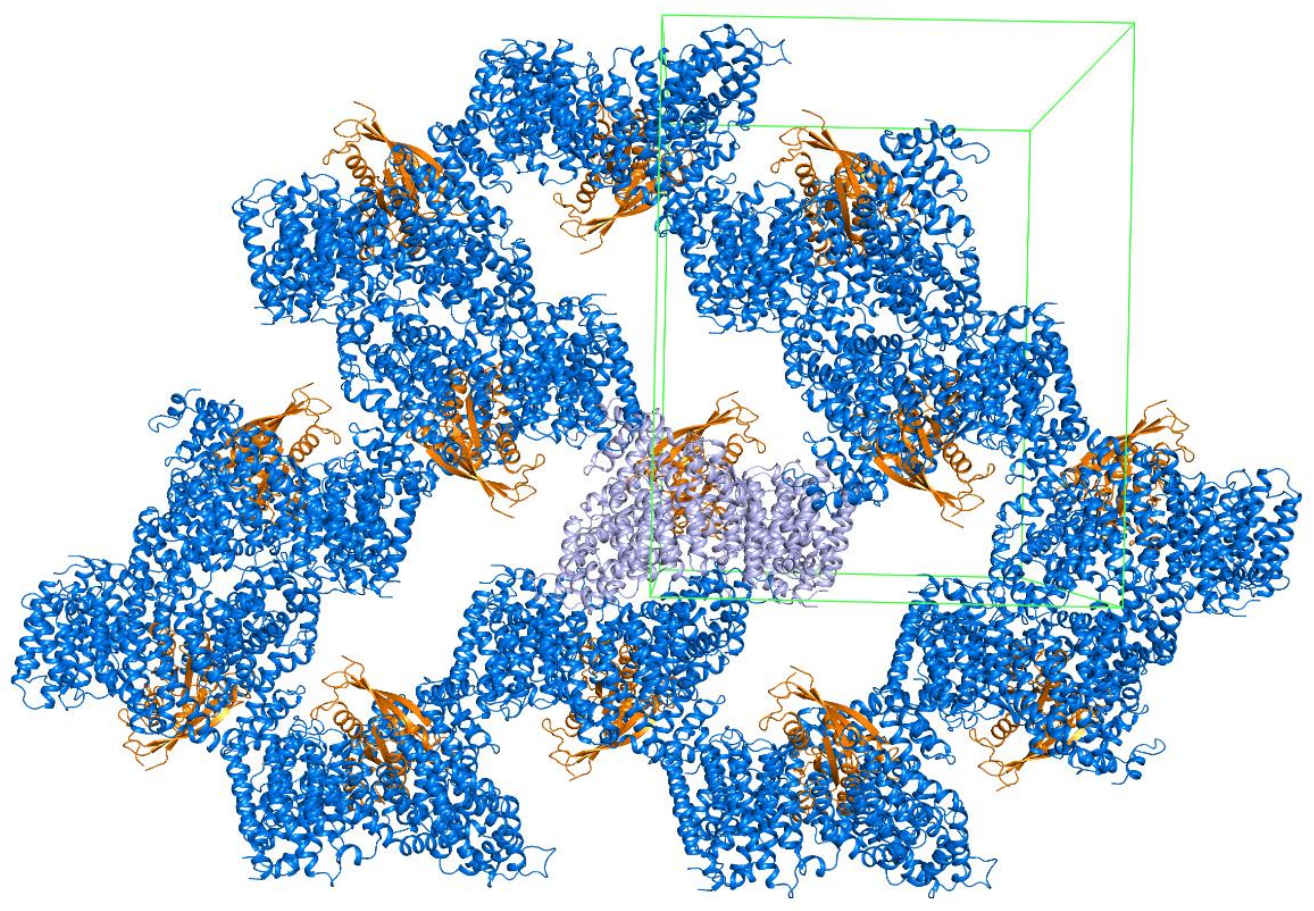

Figure 9: Crystal packing of ${ }^{H s} \mathrm{CRM} 1{ }^{-H s}$ RanGTP-LMB complex. Cartoon representation of several asymmetric units of ${ }^{H s} \mathrm{CRM} 1-{ }^{H s}$ RanGTP-LMB complex crystal along a axis of the unit cell (shown in green). RanGTP is colored orange and CRM1 is depicted in lightblue in one asymmetric unit and in marine in the other asymmetric units. 
Crystallization screening of the ${ }^{H s} C R M 1{ }^{\Delta}$-LMB complex succeeded to yield diffracting crystals belonging to the space group 1222. Interestingly, under the same conditions the crystals did not grow neither in the absence of RanGTP nor with the wild type protein. While the introduced mutations were needed to maintain CRM1 conformational stability, RanGTP was assumed to be involved in crystal contacts. However, crystal packing revealed that crystal contacts are mediated exclusively by CRM1 (Figure 9). This indicates that both the RanGTP and the introduced mutations are needed to maintain a persistent conformational stability that could only be achieved by both factors to permit crystallization of the complex. In addition to previous studies on CRM1 conformational flexibility (Dölker et al. 2013; Monecke et al. 2013, 2014), this further indicates that the exceptional flexibility of human CRM1 is a major factor that renders the human protein more challenging to crystallize than the yeast variants (section 3.3.1).

\subsection{Molecular docking predicts a similar binding mode of C3 and C6 to SINEs KPT compounds}

Compounds C3, C6 and C10 were found to inhibit CRM1-dependent nuclear export in several cancer cell lines. However, their molecular target and mechanism remained to be elucidated (Fetz et al. 2009). This study revealed that all three compounds were able to dissociate CRM1 PKI $\Phi^{0}$ Leu NES interaction in a concentration dependent fashion (section 2.3.4). Furthermore, their binding was shown to be dependent on Cys528, which indicated their mechanism of inhibition by the direct blockage of the NES binding cleft. Molecular docking predicted the binding of C3 and $\mathrm{C} 6$ to be centered around Cys528 (section 3.3.3), which suggested a similar binding mode to KPT SINE compounds (section 2.3.3). SINE compounds share a common trifluoromethyl phenyl triazole scaffold with a Michael addition acceptor side chain (Chapter 1 - table 1 ) that permits the covalent junction to Cys528 (G. Gravina et al. 2014; Inoue et al. 2013; Lapalombella et al. 2012; Sendino et al. 2018; Sun et al. 2016; Zhang et al. 2013). The structural alignment of KPT251 bound to ${ }^{S c} \mathrm{CRM} 1$ with the docked $\mathrm{C} 3$ and $\mathrm{C} 6$ compounds to ${ }^{H s} \mathrm{CRM} 1$ (Figure 10) reveals multiple similar binding properties. All three compounds C6, C3, and KPT251 occupy a similar region in the upper half of the NES-binding cleft. The binding region of these three ligands covers approximately 40 $\%$ of the hydrophobic cleft surface which results in the blockage of 3 out of 5 hydrophobic pockets $(\Phi 2-\Phi 4)$ that serve as docking sites for the NES peptide critical hydrophobic residues (Figure 10 $B, C$, and D). Furthermore, this alignment shows that predicted binding conformation of C6 in ${ }^{H s} \mathrm{CRM} 1$ is highly similar to the binding conformation of KPT521 in ${ }^{5 c} \mathrm{CRM} 1$, although both compounds are shifted by about 1.3 A relative to each other (Figure $10 \mathrm{C}$ ). In contrast, C3, which 
binds in the same region, reveals larger differences (Figure $10 \mathrm{~B}$ ). This is most likely due to structural dissimilarity of C3 when compared to C6 and KPT251 (Figure 10 A).
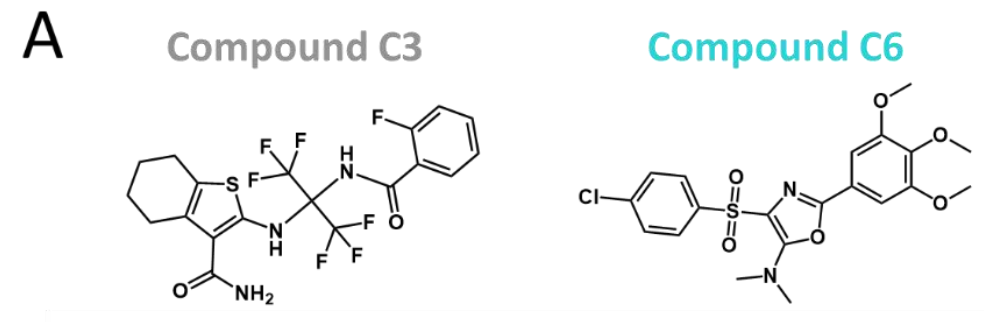

\section{KPT251}
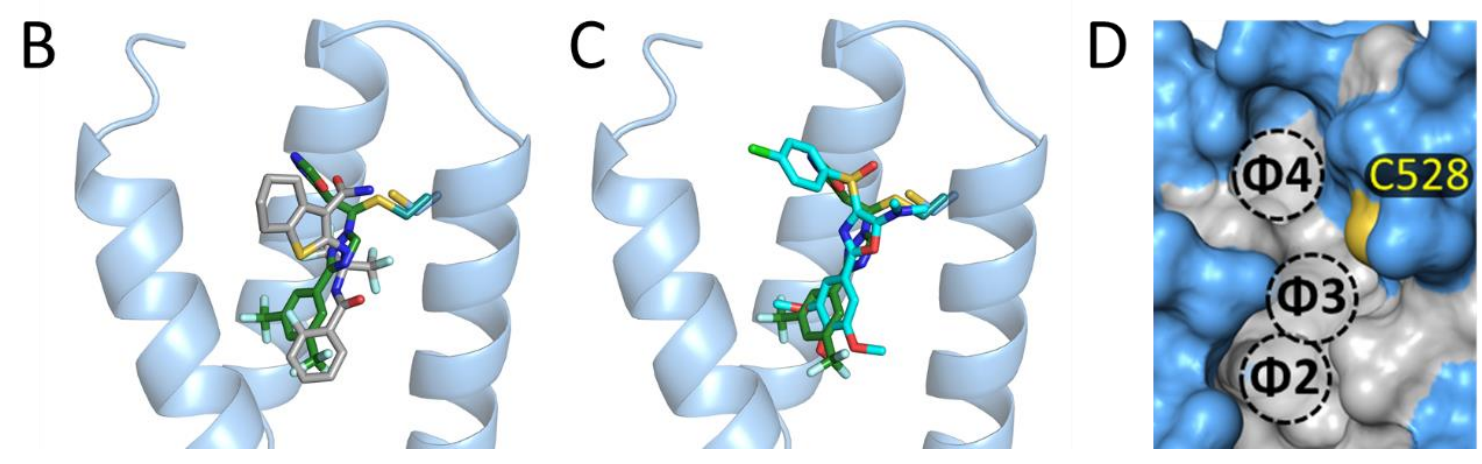

Figure 10: Structural alignment of docked C3 and C6 with KPT251 bound to ${ }^{S c}$ CRM1. (A) chemical drawings of the compounds C3, C6, and KPT251. Superposition of KPT251 (dark green) with C3 (grey) and $\mathrm{C6}$ (cyan) in (B) and (C) respectively. The compounds as well as the Cys528 of ${ }^{H s} \mathrm{CRM} 1$ and Cys539 of ${ }^{S c} \mathrm{CRM} 1$ are depicted as sticks. (D) Surface representation of the upper part of ${ }^{H s} \mathrm{CRM} 1$ NES-binding cleft showing the respective $\Phi$ pockets (Ф2 - Ф4) that are involved in NES peptide binding. Hydrophobic regions are colored grey.

Nevertheless, while the reactivity of SINE compounds is dependent on Michael type addition, the performed docking calculations can't predict potential reactivity or modifications of the tested compounds towards Cys528 (Trott and Olson 2010). Furthermore, during docking calculation the sulfur of the cysteine generates an energy barrier (repulsive interaction) that restricts the predicted position of the compounds to a certain distance from the reactive cysteine. This might result in a less reliable prediction in case of compounds known to form a covalent adduct to Cys528 like classical NES inhibitors. Therefore, docking simulations in this context provide a probable insight into binding orientation and position within the hydrophobic cleft. However, the exact binding conformation and the atomic interactions should be verified experimentally. 


\subsection{Alternative mechanisms of CRM1 mediated nuclear export inhibition}

\subsubsection{Allosteric modulation of the NES-binding cleft}

All previously studied CRM1 inhibitors share a common mechanism that is based on interfering CRM1-cargo interactions by the direct blockage of the NES-binding cleft (Dickmanns et al. 2015; Sendino et al. 2018; Sun et al. 2016). Very recently the compounds DP2392-E10 was predicted by in silico docking to bind in the region between HEAT repeats 9 and 10 (Chutiwitoonchai et al. 2017). In this study, DP2392-E10 interference with CRM1 interactions was revealed to be independent on Cys528 (section 2.3.5), which further strengthened its predicted binding outside the NES-binding cleft. In addition, when tested at similar concentrations to C3 and C10, DP2392E10 could dissociate the binding of the exceptional CRM1 cargo SPN1 that binds CRM1 in multiple regions besides its NES peptide (Chapter 2 - figure 4). This further implies DP2392-E10-based inhibition mechanism to be of an allosteric nature and independent on direct blockage of the NES cleft which, in the performed assay, requires the competition with tight SPN1 interactions (section 2.3.5). During this study docking simulations have been performed under different settings and using the atomic coordinates derived from experimentally determined crystal structure of inhibitor-bound human CRM1 ( ${ }^{H s} \mathrm{CRM} 1-{ }^{H s}$ RanGTP-LMB complex). Docking calculations of DP2392-E10 with different settings defined a binding region at the upper side of HEAT9 and at the base of the acidic loop (section 3.3.5). Furthermore, predicted binding conformation suggest a unique non-covalent interaction mode that involves several residues of HEAT9 loop which extends to form the acidic loop (section 3.3.5). Docking calculations supported by the experimental findings suggest a novel mechanism of CRM1 inhibition that is based on interfering exportin - cargo interactions by the allosteric modulation of the NES-binding cleft. In the case of DP2392-E10, this can be achieved by altering the conformation of the acidic loop which is a key structural element in CRM1 conformational transition and cargo release (Dölker et al. 2013; Koyama and Matsuura 2010; Monecke et al. 2014). These findings define a new aspect of CRM1 inhibition that is not based on reactive compounds. The development of CRM1 targeting compounds with such properties outcompetes the current nuclear export inhibitors by exhibiting less toxicity during clinical evaluation.

\subsubsection{Interference with RanGTP interactions}

The nuclear export of a CRM1 cargo is strictly dependent on the formation of a ternary export complex that involves RanGTP and the cargo protein. The assembly of a stable complex is mediated by the cooperative binding of RanGTP and the cargo protein to CRM1. In the absence 
of RanGTP most cargoes exhibit low affinity towards the export receptor and therefore the export process cannot be initiated (Monecke et al. 2014). While all known CRM1 inhibitors interfere with cargo binding, nuclear transport can also be inhibited by interfering transport receptor - RanGTP interaction (Hill et al. 2014). Computational analysis of potential ligand binding sites using human CRM1 from the complex ${ }^{H s}$ CRM1- ${ }^{H s}$ RanGTP-LMB crystal structure identified more than 40 potential binding sites outside the NES-binding cleft (section 3.3.4). Most sites were distributed at the outer surface of the protein, whereas few sites were identified at the inner surface of HEAT repeats 2 and 3 . This region is known as the CRIME domain and it represents the binding site of RanGTP (Petosa et al. 2004). The identified potential ligand binding site at the CRIME domain indicates for the possibly of developing CRM1 inhibitors that prevent or disturb RanGTP binding. Such an approach has been already applied for the identification of nuclear import inhibitors that affect the importin $\beta$ - RanGTP interaction (Hintersteiner et al. 2010; Soderholm et al. 2011). Karyostatin $1 \mathrm{~A}$ is a compound that inhibits importin $\beta$ mediated nuclear import of GFP-NFAT in HeLa cells. Karyostatin $1 \mathrm{~A}$ was initially identified in an affinity-based screening by confocal nanoscanning. Further analysis revealed that Karyostatin $1 \mathrm{~A}$ acts by the selective binding to importin $\beta$ that disrupts its interactions with RanGTP (Hintersteiner et al. 2010). In another study, FRET-based high throughput screen that detects the interaction between importin $\beta$ and RanGTP identified an inhibitor named importazole, a 2,4-diaminoquinazoline. Importazole mechanism is based on interfering importin $\beta$ - RanGTP interaction; it also exhibits high specificity towards importin $\beta$ and was shown to block the nuclear import in Xenopus egg extracts as well as in cultured cells (Soderholm et al. 2011). These examples provide an experimental evidence that inhibiting RanGTP interaction with the transport receptor can be a considerable approach for the development of anti-tumor and anti-viral drugs that target the nucleocytoplasmic transport machinery.

\subsubsection{Interfering CRM1 translocation through the nuclear pore complex}

Interfering the association of the export complex with the NPC represents an alternative mechanism that has not been yet addressed or considered for inhibiting CRM1 mediated nuclear export. This is most likely due to the insufficient knowledge on CRM1 interaction with the NPC from a structural perspective. The crystal structure of human CRM1 in complex with a Nup214 fragment that contains 8 FG-repeat motifs was recently published, and it is the first crystal structure that provided an insight into CRM1 interactions with the NPC (Port et al. 2015). Soon after, another structure of ${ }^{S c} \mathrm{CRM} 1$ in complex with SxFG/PxFG repeat peptide of the yeast Nup42 was released (Koyama et al. 2017). Both structures revealed that CRM1 interactions with FG- 
repeats is mediated by the binding of FG motifs as the main anchor points of FG-repeat peptides (Koyama et al. 2017; Port et al. 2015). FG motifs were shown to bind CRM1 in specific pockets that are distributed at the outer surface of the transport receptor at the $\mathrm{N}$ - and $\mathrm{C}$ - termini (section 1.2.4). This characteristic binding of FG-repeats is important to maintain the export complex stability during transportation and to allow the complex translocation through the NPC (Koyama et al. 2017; Port et al. 2015). During this study, human CRM1 from the complex ${ }^{H s}$ CRM1${ }^{H s}$ RanGTP-LMB crystal structure was subjected to computational analysis for the identification of potential ligand binding sites. Most of the identified binding sites are distributed at the outer surface of CRM1 (Chapter 3 - figure 6) which strongly indicated the possibility to inhibit the export receptor by disrupting its translocation through the NPC.
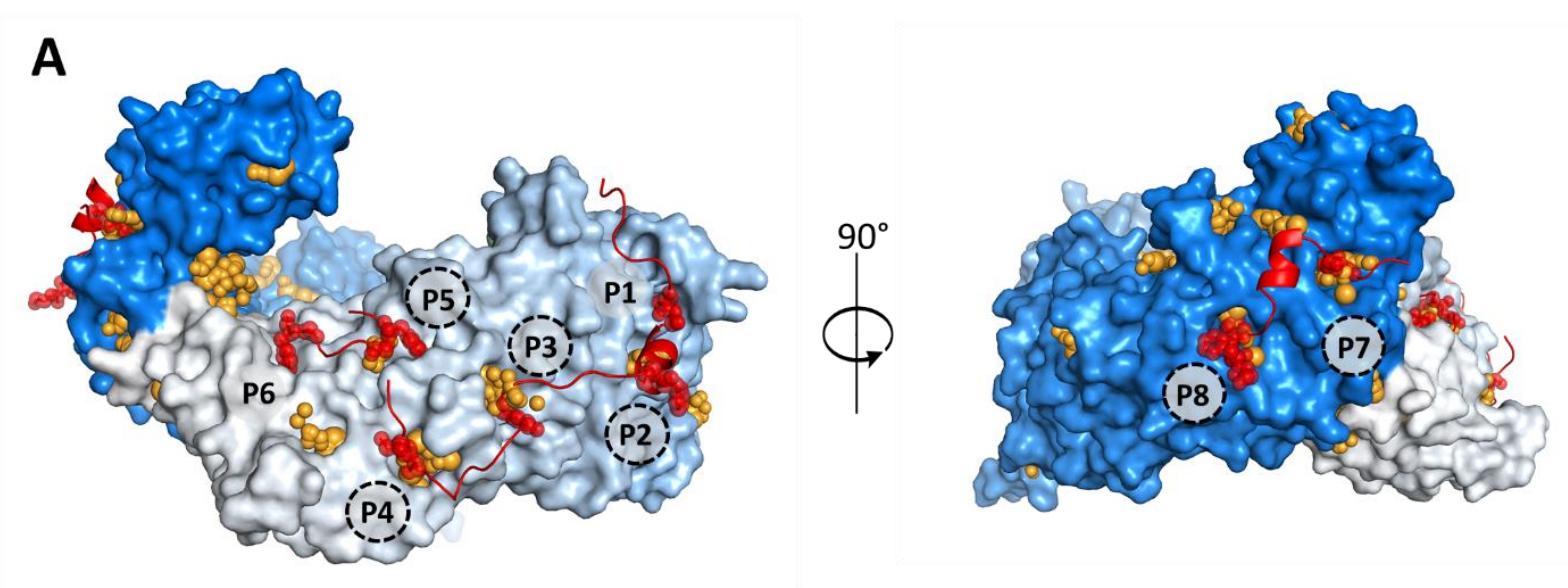

B

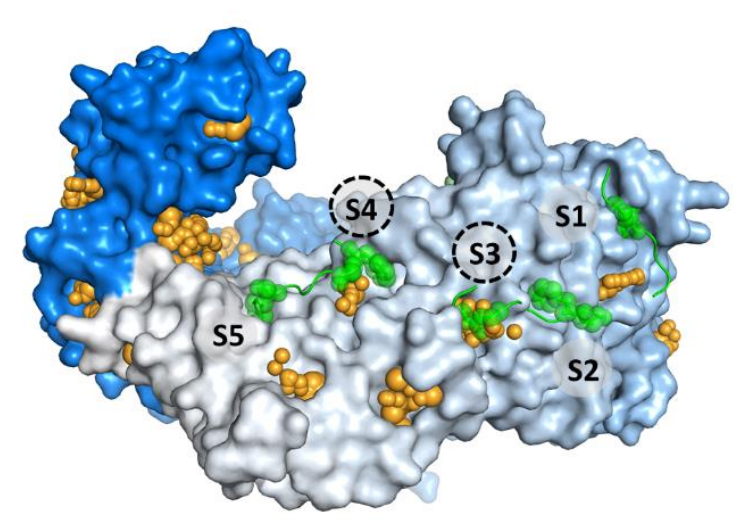

Figure 11: Structural alignment of human CRM1 showing potential ligand binding site with CRM1bound Nup214 FG repeat fragment in (A) and with CRM1-bound Nup42 FG repeat fragments in (B). binding sites (filled with orange spheres) were detected using the Alpha Site Finder function of the program MOE. FG repeat fragments are shown in cartoon representation (Nup214 in red, Nup42 in green) with FG motifs depicted as spheres. FG motif binding sites are indicated as P1-P8 for Nup214 fragment and as S1-S5 for Nup42 fragments. Binding pockets that align with potential binding sites are circled with a dashed line. 
Interestingly, the alignment of potential ligand binding sites, identified by Site Finder application available in MOE software package, with CRM1-NUPs FG-repeats complex structures unveiled overlap in case of several FG binding pockets (Figure 11) (Anon n.d.; Tøndel, Anderssen, and Drabløs 2006). Six out of eight binding pockets (P1-P8) of Nup214 (Figure 11 A) fragment FG motifs as well as two out of five binding pockets (S1-S5) of Nup42 FG motifs were identified as potential ligand binding sites at the outer surface of CRM1 (Figure $11 \mathrm{~B}$ ). Most of the identified pockets are highly conserved among distantly related species what indicates their functional importance (Koyama et al. 2017; Port et al. 2015). Furthermore, the identified pockets are suggested to be involved in the binding of other NUPs which was experimentally proven for Nup62 and RanBP3, an FG-repeat containing nucleoporin-like protein (Port et al. 2015). These findings together with the biochemical and functional analysis performed in both studies strongly indicate that the blocking of FG motifs binding pockets interferes with transport receptor translocation through the NPC. Interfering CRM1 interaction with the NPC may reduce CRM1mediated export which might be a more reliable and less toxic approach to counteract its overexpression in several cancer diseases. This concept has been already considered for the inhibition of nuclear import mediated by importin $\beta$ (Ambrus et al. 2010; Gasiorowski and Dean 2003), for which several structures with FG repeats were reported over the last two decades (Bayliss et al. 2002, 2000; Isgro and Schulten 2005; Liu and Stewart 2005). A peptidomimetic inhibitor that mimics the FXFG motif was identified as an inhibitor of the importin- $\alpha / \beta$ mediated import. (Ambrus et al. 2010) The identified peptidomimetic compound exhibited a selective inhibition of importin $\alpha / \beta$ mediated transport (Ambrus et al. 2010), indicating that this approach has potentials for developing nuclear transport inhibitors with high specificity.

\subsection{Structure-based methods in the discovery and development of CRM1 targeting drugs}

\subsubsection{Structure-based virtual screening}

A typical drug discovery and development process undergoes several stages including target selection, hit identification, lead optimization, preclinical and clinical evaluations (Hughes et al. 2011). Mechanisms of CRM1 inhibition explained in the previous section are based on developing inhibitors that bind in specific sites distributed at the protein surface. Structure-based virtual screening, a method based on computational modeling and docking, is applied at early stages of drug discovery for the identification of compounds that might bind in a specific pocket within an enzyme or a receptor 'hits'. Virtual screening has been used as a principle technique in drug 
discovery over the last decade as it has proven to be more time- and cost- efficient than other methods applied for hits identification (Kontoyianni 2017; Lionta et al. 2014). During virtual screening a library of compounds is docked in a binding pocket of a protein, for example, a potential ligand binding site in CRM1. A scoring function is applied for evaluating the binding efficiency (binding free energy) for number of solutions (poses) per each compound. Top-ranked compounds are usually redocked and scored for extra precision and a subset is then chosen for experimental approval of positive hits (Kontoyianni 2017; Lionta et al. 2014). Virtual screening has been already applied for CRM1 in the NES-binding cleft and resulted in the development of SINE KPT compounds (Kalid et al. 2012), which showed a pronounced progress in the development of CRM1 targeting anti-tumor drugs (Wang and Liu 2019). Consensus induced fit docking was performed using CRM1 crystal structure available at that time (cargo bound; PDB ID: 3GJX). The applied docking approach was initially tested on $\mathrm{N}$-azolylacrylate analogs as well as CBS9106 and afterwards was applied for screening a library of 250000 compounds. Based on the docking results a total of 402 compounds were selected for experimental evaluation. 17 of these compounds exhibited a binding affinity below $100 \mu \mathrm{M}$ which conformed the efficiency of this approach for the identification of CRM1 promising targeting hits (Kalid et al. 2012). The complex ${ }^{H s}$ CRM1- ${ }^{H s}$ RanGTP-LMB crystal structure obtained in this study is the first structure of inhibitor-bound human CRM1 (section 2.3.1). The improvement and the development of several docking approaches over the last decade (Kontoyianni 2017) together with the obtained inhibitor-bound human CRM1 crystal structure provide an efficient and reliable framework for the development of CRM1 inhibitors that target potential sites outside the NES-binding cleft. Furthermore, the fluorescence polarization competition assay, presented in this study (section 2.2.7) is an efficient tool for the experimental evaluation of identified hit that may allosterically inhibit cargo binding or interfere CRM1-RanGTP interaction.

\subsubsection{Crystallographic fragment screening}

Crystallographic fragment screening is a structure-guided approach that is applied at early stages of drug discovery for the identification of lead compounds (Rees et al. 2004). The method is based on co-crystallization or socking target protein crystals with high concentrations of small molecule compounds (typically MW 110-250 Da) that represent fragments of potential drugs. Positive hits are identified by the visualizing their specific binding in electron density map based on the analysis of X-ray diffraction data obtained from prepared crystals (Badger 2012; Thomas et al. 2019). Although this approach is more time consuming and labor intensive than virtual screening or high throughput screening (HTS), it offers several unique advantages over most other 
techniques (Badger 2012; Patel, Bauman, and Arnold 2014). First, crystallographic screening is a highly sensitive approach that can detect ligands with binding affinity range of sub-nanomolar to millimolar. Second, positive hits are identified with a three-dimensional perspective of their binding confirmation with respect to the target pocket which provide valuable knowledge on potential optimization by fragment expansion, merging, or linking (Patel et al. 2014). Furthermore, crystallographic fragment screening shows few false positive hits in comparison with HTS or virtual screening (Badger 2012).

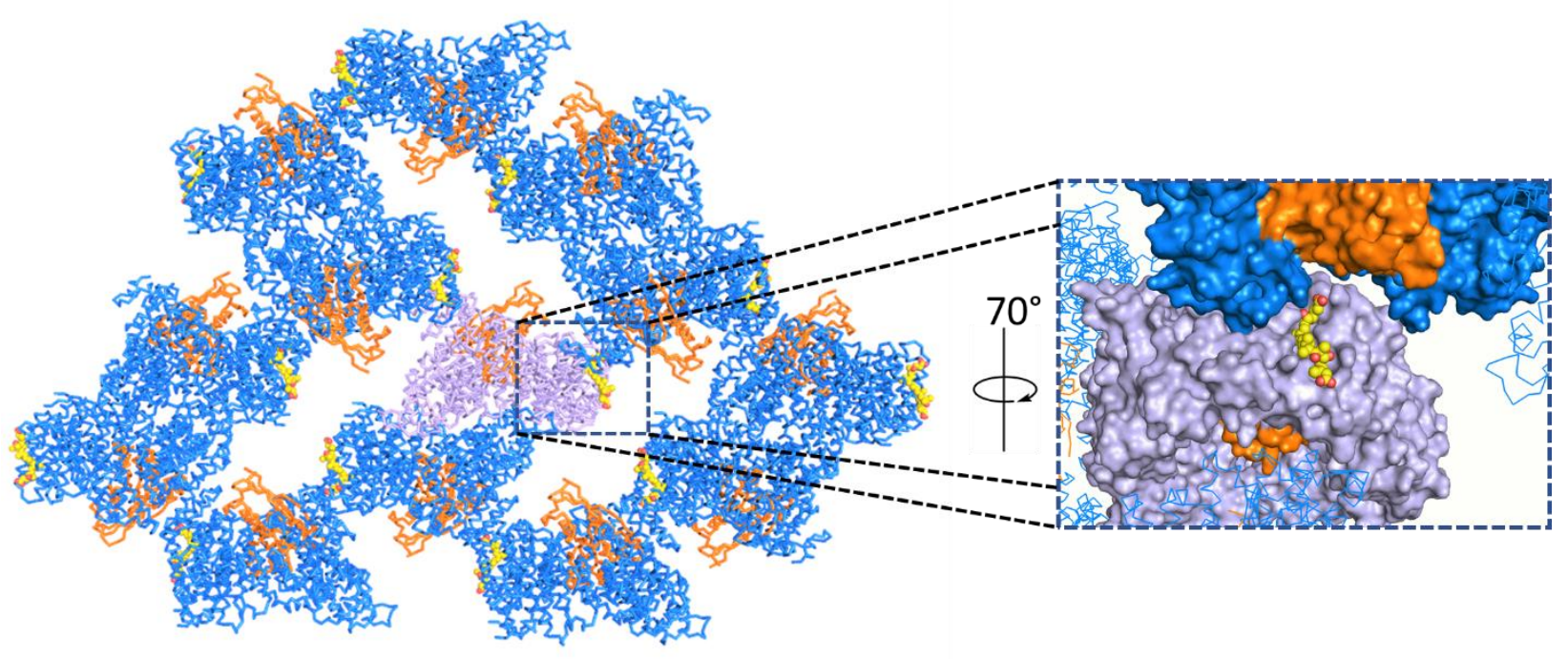

Figure 12: Crystal packing of ${ }^{H s} \mathrm{CRM} 1-{ }^{H s}$ RanGTP-LMB showing the NES-binding cleft is exposed to solvent channels. Ribbon representation of several asymmetric units showing CRM1 depicted in lightblue in one asymmetric unit and in marine in the other asymmetric units. RanGTP is depicted as orange ribbons and $L M B$ is shown as spheres. In the left panel a surface representation of two neighboring molecules showing that the NES cleft, occupied by the LMB, as well as most of the outer side surface of CRM1 are not involved in crystal contacts and exposed to the solvent channels.

Although co-crystallization with yeast ${ }^{S c} \mathrm{CRM} 1$ has been already applied for the structural characterization of known CRM1 inhibitors, crystallographic fragment screening for alternative novel inhibitors has not been yet reported in literature. This is most likely due to the crystallographic requirements of this approach. In order for a target protein to be feasible for crystallographic fragment screening, it has to produce crystals that diffract to beyond medium resolution (around $2.5 \AA$ ) and provide an unblocked access to potential target sites (Badger 2012). Interestingly, the crystallization approach of human CRM1 that is developed in this study (section 3.3.1), although it requires further improvement (section 3.4), demonstrates strong potentials to be developed for crystallographic fragment screening. Crystal packing of ${ }^{H s} \mathrm{CRM1}{ }^{H s}$ RanGTP-LMB complex crystal shows that the crystal contacts are mainly mediated by the bottom surfaces of 
neighbouring CRM1 molecules and thus causing the majority of the outer surface of the protein to be exposed to solvent channels. This unique arrangement of molecules within the crystal lattice renders the NES-binding cleft as well as most identified potential ligand binding sites solvent exposed and hence accessible by screened compounds (Figure 12). In addition, the majority of crystals yielded by this approach diffracted down to 3.5 - $3 \AA$ A before further treatment or optimization (section 3.3.1), suggesting that applying optimizations may improve their diffraction potentials to acceptable levels for crystallographic based screening. These unique properties of the obtained crystals render the developed approach a valuable tool not only for crystallographic screening but also as a complementary tool for other methods applied in drug discovery.

In summary, the work presented in this thesis provide a biochemical as well as an integrative experimental and computational structural insights into human CRM1 inhibition. This work revealed significant differences in inhibitors' binding properties towards human and yeast ${ }^{S c} \mathrm{CRM} 1$ and thus demonstrates the importance of applying inhibitor studies on the human protein. Furthermore, the work presented here provide a general perspective on potential CRM1inhibitory mechanisms and possible structure-based methods for the development of novel nuclear export inhibiting drugs. Finally, the complex ${ }^{H s} \mathrm{CRM} 1-{ }^{H s}$ RanGTP-LMB crystal structure obtained during this work represents a valuable tool for future studies on CRM1-targeting antitumor and anti-viral drug design. 


\section{Outlook}

The work described in thesis focused on the biochemical and structural characterization of CRM1 inhibitors using the human protein, which was observed to be more challenging to crystallize than the yeast variants. Using LMB together with genetically modifying human CRM1 succeeded to gain the crystal structure of ${ }^{H s} \mathrm{CRM} 1-{ }^{H s}$ RanGTP-LMB complex presented in this thesis. The obtained structure revealed several potentials that render the developed approach promising for crystallographic - based drug design. However, when the same approach was applied for the crystallization of the compound $\mathrm{C} 6$, the binding of the compound was prevented by the covalent modification of Cys 528 by BME. Alternatively, computational docking was performed to predict the binding conformation of $\mathrm{C} 3, \mathrm{C} 6$, and $\mathrm{C} 10$. The docking method could only provide information on possible binding conformation, while the exact binding mode and potential reactivity with Cys528 are still to be elucidated experimentally. For this purpose, BME, which was introduced during protein purification as a reducing agent, must be excluded throughout the entire purification and crystallization procedures. TCEP, a reducing agent that is stable at a broader $\mathrm{pH}$ range compared to BME (Getz et al. 1999), can be applied as an alternative to maintain protein stability and prevent its aggregation during preparation. Second, although the diffraction power of the crystals can be improved by stepwise dehydration, improving the crystallization buffer conditions (for example by additive screening) might be a more efficient approach for robust production of complex crystals with improved diffraction power. Upon optimization, cocrystallization can be applied to gain structural information on C3, C6, and C10 binding to CRM1.

With respect to DP2392-E10, although it is predicted to bind outside the NES-binding cleft, it did not crystallize in complex with CRM1 and RanGTP under the same conditions as LMB or C6. The complex crystallization could be hindered by possible conformational changes that might be induced by DP2392-E10 binding. This implies that for this compound it might be necessary to perform a general screening to identify conditions that permit the crystallization of the complex in its altered conformation. Nevertheless, the predicted binding site and interactions can be verified experimentally by introducing several mutations in the suggested pocket and test the compound binding by the established fluorescence competition assay. It is very important in this experiment to test the stability and functionality of CRM1 after each mutation. This can be assessed by following the binding of the fluorescently labeled NES peptide and observe if its binding affinity towards the mutant is different from the wild type protein. 
Lastly, in this thesis several alternative mechanisms were proposed for the inhibition of CRM1mediated nuclear export based on the identification of multiple potential ligand binding sites. Virtual screening can be performed using the inhibitor - bound human CRM1 structure obtained in this study as template for the identification of hit compounds in a specific potential binding site. The binding of the identified candidates can be verified experimentally using the fluorescence polarization competition assay described here. Furthermore, the crystallization approach, once it is optimized, can be applied for crystallographic based fragment screening as well as for the structural characterization of hit compounds identified by virtual screening. 


\section{References}

Adachi, Y. and M. Yanagida. 1989. "Higher Order Chromosome Structure Is Affected by Cold-Sensitive Mutations in a Schizosaccharomyces Pombe Gene Crm1+ Which Encodes a 115-KD Protein Preferentially Localized in the Nucleus and Its Periphery." The Journal of Cell Biology 108(4):11951207.

Aggarwal, Bharat B., Chitra Sundaram, Nikita Malani, and Haruyo Ichikawa. 2007. "Curcumin: The Indian Solid Gold." Advances in Experimental Medicine and Biology 595:1-75.

Alexander, K. and P. W. Hinds. 2001. "Requirement for P27KIP1 in Retinoblastoma Protein-Mediated Senescence." Molecular and Cellular Biology 21(11):3616-31.

Ambrus, Géza, Landon R. Whitby, Eric L. Singer, Oleg Trott, Euna Choi, Arthur J. Olson, Dale L. Boger, and Larry Gerace. 2010. "Small Molecule Peptidomimetic Inhibitors of Importin $\alpha / \beta$ Mediated Nuclear Transport." Bioorganic and Medicinal Chemistry 18(21):7611-20.

Andrade, Miguel A. and Peer Bork. 1995. "HEAT Repeats in the Huntington's Disease Protein." Nature Genetics 11(2):115-16.

Andrade, Miguel A., Carolina Perez-Iratxeta, and Chris P. Ponting. 2001. "Protein Repeats: Structures, Functions, and Evolution." Journal of Structural Biology 134(2-3):117-31.

Anon. n.d. "A Phase 1 Trial of a Novel XPO1 Inhibitor in Patients With Advanced Solid Tumors - Full Text View - ClinicalTrials.Gov." Retrieved March 5, 2020a (https://clinicaltrials.gov/ct2/show/NCT02667873).

Anon. n.d. "Molecular Operating Environment (MOE) | MOEsaic | PSILO." Retrieved January 20, 2020b (https://www.chemcomp.com/Products.htm).

Aramburu, Iker Valle and Edward A. Lemke. 2017. "Floppy but Not Sloppy: Interaction Mechanism of FGNucleoporins and Nuclear Transport Receptors." Seminars in Cell and Developmental Biology 68:34-41.

Arnaoutov, Alexei, Yoshiaki Azuma, Katharina Ribbeck, Jomon Joseph, Yekaterina Boyarchuk, Tatiana Karpova, James McNally, and Mary Dasso. 2005. "Crm1 Is a Mitotic Effector of Ran-GTP in Somatic Cells." Nature Cell Biology 7(6):626-32.

Azmi, Asfar S., Amro Aboukameel, Bin Bao, Fazlul H. Sarkar, Philip A. Philip, Michael Kauffman, Sharon Shacham, and Ramzi M. Mohammad. 2013. "Selective Inhibitors of Nuclear Export Block Pancreatic Cancer Cell Proliferation and Reduce Tumor Growth in Mice." Gastroenterology 144(2):447-56.

Badger, John. 2012. "Crystallographic Fragment Screening." Methods in Molecular Biology 841:161-77.

Baer, Richard and Thomas Ludwig. 2002. "The BRCA1/BARD1 Heterodimer, a Tumor Suppressor Complex with Ubiquitin E3 Ligase Activity." Current Opinion in Genetics and Development 12(1):8691.

Bartholomeusz, Geoffrey, Yadi Wu, Mohamad Ali Seyed, Weiya Xia, Ka Yin Kwong, Gabriel Hortobagyi, and Mien Chie Hung. 2006. "Nuclear Translocation of the Pro-Apoptotic Bcl-2 Family Member Bok Induces Apoptosis." Molecular Carcinogenesis 45(2):73-83.

Bayliss, Richard, Trevor Littlewood, and Murray Stewart. 2000. "Structural Basis for the Interaction 
between FxFG Nucleoporin Repeats and Importin- $\beta$ in Nuclear Trafficking." Cell 102(1):99-108.

Bayliss, Richard, Trevor Littlewood, Lisa A. Strawn, Susan R. Wente, and Murray Stewart. 2002. "GLFG and FxFG Nucleoporins Bind to Overlapping Sites on Importin- $\beta$. ." Journal of Biological Chemistry 277(52):50597-606.

Beals, Chan R., Neil A. Clipstone, Steffan N. Ho, and Gerald R. Crabtree. 1997. "Nuclear Localization of NF-ATc by a Calcineurin-Dependent, Cyclosporin- Sensitive Intramolecular Interaction." Genes and Development 11(7):824-34.

Benzeno, Sharon and J. Alan Diehl. 2004. "C-Terminal Sequences Direct Cyclin D1-CRM1 Binding." Journal of Biological Chemistry 279(53):56061-66.

Besson, Arnaud, Richard K. Assoian, and James M. Roberts. 2004. "Regulation of the Cytoskeleton: An Oncogenic Function for CDK Inhibitors?" Nature Reviews Cancer 4(12):948-55.

Bezerra, Daniel P., Claudia Pessoa, Manoel O. De Moraes, Nicolau Saker-Neto, Edilberto R. Silveira, and Leticia V. Costa-Lotufo. 2013. "Overview of the Therapeutic Potential of Piplartine (Piperlongumine)." European Journal of Pharmaceutical Sciences 48(3):453-63.

Bischoff, F. R., H. Krebber, E. Smirnova, W. Dong, and H. Ponstingl. 1995. “Co-Activation of RanGTPase and Inhibition of GTP Dissociation by Ran-GTP Binding Protein RanBP1." The EMBO Journal 14(4):705-15.

Bischoff, F. Ralf and Herwig Ponstingl. 1991. "Catalysis of Guanine Nucleotide Exchange on Ran by the Mitotic Regulator RCC1." Nature 354(6348):80-82.

Bonazzi, Simone, Oliv Eidam, Stephan Güttinger, Jean-Yves Wach, Ivo Zemp, Ulrike Kutay, and Karl Gademann. 2010. "Anguinomycins and Derivatives: Total Syntheses, Modeling, and Biological Evaluation of the Inhibition of Nucleocytoplasmic Transport." Journal of the American Chemical Society 132(4):1432-42.

Bonner, William M. 1975. "Protein Migration into Nuclei: I. Frog Oocyte Nuclei in Vivo Accumulate Microinjected Histones, Allow Entry to Small Proteins, and Exclude Large Proteins." Journal of Cell Biology 64(2):421-30.

Burkhart, Deborah L. and Julien Sage. 2008. "Cellular Mechanisms of Tumour Suppression by the Retinoblastoma Gene." Nature Reviews Cancer 8(9):671-82.

Burzlaff, A., M. Kalesse, C. Kasper, and T. Scheper. 2003. "Multi Parameter in Vitro Testing of Ratjadone Using Flow Cytometry." Applied Microbiology and Biotechnology 62(2-3):174-79.

Cao, Shuai, Xiaoling Liu, Maorong Yu, Jing Li, Xiaojuan Jia, Yuhai Bi, Lei Sun, George F. Gao, and Wenjun Liu. 2012. "A Nuclear Export Signal in the Matrix Protein of Influenza A Virus Is Required for Efficient Virus Replication." Journal of Virology 86(9):4883-91.

Cao, Yuan and Xin-yong Liu. 2007. "HIV-1 Rev and Related Inhibitors." Yao Xue Xue Bao = Acta Pharmaceutica Sinica 42(4):347-51.

Chen, Janice, Christopher L. Brooks, Peter McDonald, Jonathan D. Schwartz, Keiichi Sakakibara, Naoya Saito, Takuji Sato, Takumi Kawabe, and Eric Keith Rowinsky. 2015. "Preclinical Activity of SL-801, a Reversible Inhibitor of Exportin-1 (XPO1)/Chromosome Region Maintenance-1 (CRM1) in Solid and Hematologic Cancers." Journal of Clinical Oncology 33(15_suppl):e13543-e13543. 
Chen, Janice, Christopher L. Brooks, Peter McDonald, Jonathan D. Schwartz, Rebecca S. Schneider, Keiichi Sakakibara, Naoya Saito, Takuji Sato, Takumi Kawabe, and Eric K. Rowinsky. 2015. "SL-801, a Novel, Reversible Inhibitor of Exportin-1 (XPO1) / Chromosome Region Maintenance-1 (CRM1) with Broad and Potent Anti-Cancer Activity." Blood 126(23):4433-4433.

Chook, Yuh Min, Gino Cingolani, Elena Conti, Murray Stewart, Ingrid Vetter, and Alfred Wittinghofer. 1999. "Pictures in Cell Biology Structures of Nuclear-Transport Components." Trends in Cell Biology 9(8):310-11.

Chutiwitoonchai, Nopporn, Takafumi Mano, Michinori Kakisaka, Hirotaka Hironori Sato, Yasumitsu Kondoh, Hiroyuki Osada, Osamu Kotani, Masaru Yokoyama, Hirotaka Hironori Sato, and Yoko Aida. 2017. "Inhibition of CRM1-Mediated Nuclear Export of Influenza A Nucleoprotein and Nuclear Export Protein as a Novel Target for Antiviral Drug Development." Virology 507(April):32-39.

Conforti, Fabio, Yisong Wang, Jose A. Rodriguez, Anna Teresa Alberobello, Yu Wen Zhang, and Giuseppe Giaccone. 2015. "Molecular Pathways: Anticancer Activity by Inhibition of Nucleocytoplasmic Shuttling." Clinical Cancer Research 21(20):4508-13.

Cong, Feng and Harold Varmus. 2004. "Nuclear-Cytoplasmic Shuttling of Axin Regulates Subcellular Localization of $\beta$-Catenin." Proceedings of the National Academy of Sciences of the United States of America 101(9):2882-87.

Connor, Michael K., Rouslan Kotchetkov, Sandrine Cariou, Ansgar Resch, Rafaella Lupetti, Richard G. Beniston, Frauke Melchior, Ludger Hengst, and Joyce M. Slingerland. 2003. "CRM1/Ran-Mediated Nuclear Export of P27Kip1 Involves a Nuclear Export Signal and Links P27 Export and Proteolysis." Molecular Biology of the Cell 14(1):201-13.

Conti, E. and E. Izaurralde. 2001. "Nucleocytoplasmic Transport Enters the Atomic Age." Current Opinion in Cell Biology 13(3):310-19.

Cook, Atlanta, Fulvia Bono, Martin Jinek, and Elena Conti. 2007. "Structural Biology of Nucleocytoplasmic Transport." Annual Review of Biochemistry 76(1):647-71.

Crabtree, Gerald R. and Eric N. Olson. 2002. "NFAT Signaling: Choreographing the Social Lives of Cells." Cell 109.

Craig, Errol, Zhi-Kai Zhang, Kelvin P. Davies, and Ganjam V Kalpana. 2002. "A Masked NES in INI1/HSNF5 Mediates HCRM1-Dependent Nuclear Export: Implications for Tumorigenesis." The EMBO Journal 21(1-2):31-42.

Cronshaw, Janet M., Andrew N. Krutchinsky, Wenzhu Zhang, Brian T. Chait, and Michae L. J. Matunis. 2002. "Proteomic Analysis of the Mammalian Nuclear Pore Complex." Journal of Cell Biology 158(5):915-27.

Daelemans, Dirk, Elena Afonina, Jakob Nilsson, Gudrun Werner, Jorgen Kjems, Erik De Clercq, George N. Pavlakis, and Anne-Mieke Vandamme. 2002. "A Synthetic HIV-1 Rev Inhibitor Interfering with the CRM1-Mediated Nuclear Export." Proceedings of the National Academy of Sciences of the United States of America 99(22):14440-45.

Denning, Daniel P., Samir S. Patel, Vladimir Uversky, Anthony L. Fink, and Michael Rexach. 2003. "Disorder in the Nuclear Pore Complex: The FG Repeat Regions of Nucleoporins Are Natively Unfolded." Proceedings of the National Academy of Sciences of the United States of America 100(5):2450-55. 
Dickmanns, Achim, Thomas Monecke, and Ralf Ficner. 2015. Structural Basis of Targeting the Exportin CRM1 in Cancer. Vol. 4.

Dölker, Nicole, Clement E. Blanchet, Béla Voß, David Haselbach, Christian Kappel, Thomas Monecke, Dmitri I. Svergun, Holger Stark, Ralf Ficner, Ulrich Zachariae, Helmut Grubmüller, and Achim Dickmanns. 2013. "Structural Determinants and Mechanism of Mammalian CRM1 Allostery." Structure 21(8):1350-60.

Dölker, Nicole, Ulrich Zachariae, and Helmut Grubmüller. 2010. "Hydrophilic Linkers and Polar Contacts Affect Aggregation of FG Repeat Peptides." Biophysical Journal 98(11):2653-61.

Dong, Xiuhua, Anindita Biswas, Katherine E. Süel, Laurie K. Jackson, Rita Martinez, Hongmei Gu, and Yuh Min Chook. 2009. "Structural Basis for Leucine-Rich Nuclear Export Signal Recognition by CRM1." Nature 458(7242):1136-41.

Dou, Dan, Rebecca Revol, Henrik Östbye, Hao Wang, and Robert Daniels. 2018. "Influenza A Virus Cell Entry, Replication, Virion Assembly and Movement." Frontiers in Immunology 9(JUL).

Elton, D., M. Simpson-Holley, K. Archer, L. Medcalf, R. Hallam, J. McCauley, and P. Digard. 2001. "Interaction of the Influenza Virus Nucleoprotein with the Cellular CRM1-Mediated Nuclear Export Pathway." Journal of Virology 75(1):408-19.

Engel, Roxane, Nikola I. Valkov, Jana L. Gump, Lori Hazlehurst, William S. Dalton, and Daniel M. Sullivan. 2004. "The Cytoplasmic Trafficking of DNA Topoisomerase Il $\alpha$ Correlates with Etoposide Resistance in Human Myeloma Cells." Experimental Cell Research 295(2):421-31.

Etchin, J., Q. Sun, A. Kentsis, A. Farmer, Z. C. Zhang, T. Sanda, M. R. Mansour, C. Barcelo, D. McCauley, M. Kauffman, S. Shacham, A. L. Christie, A. L. Kung, S. J. Rodig, Y. M. Chook, and A. T. Look. 2013. "Antileukemic Activity of Nuclear Export Inhibitors That Spare Normal Hematopoietic Cells." Leukemia.

Etchin, Julia, Takaomi Sanda, Marc R. Mansour, Alex Kentsis, Joan Montero, Bonnie T. Le, Amanda L. Christie, Dilara Mccauley, Scott J. Rodig, Michael Kauffman, Sharon Shacham, Richard Stone, Anthony Letai, Andrew L. Kung, and A. Thomas Look. 2013. "KPT-330 Inhibitor of CRM1 (XPO1)Mediated Nuclear Export Has Selective Anti-Leukaemic Activity in Preclinical Models of T-Cell Acute Lymphoblastic Leukaemia and Acute Myeloid Leukaemia." British Journal of Haematology 161(1):117-27.

Fagan-Solis, Katerina D., Brian T. Pentecost, Joseph M. Gozgit, Brooke A. Bentley, Sharon M. Marconi, Christopher N. Otis, Douglas L. Anderton, Sallie Smith Schneider, and Kathleen F. Arcaro. 2014. "SKP2 Overexpression Is Associated with Increased Serine 10 Phosphorylation of P27 (PSer10p27) in Triple-Negative Breast Cancer." Journal of Cellular Physiology 229(9):1160-69.

Faustino, R. S., T. J. Nelson, A. Terzic, and C. Perez-Terzic. 2007. "Nuclear Transport: Target for Therapy." Clinical Pharmacology and Therapeutics 81(6):880-86.

Fetz, Verena, Shirley K. Knauer, Carolin Bier, Jens Peter von Kries, and Roland H. Stauber. 2009. "Translocation Biosensors ? Cellular System Integrators to Dissect CRM1-Dependent Nuclear Export by Chemicogenomics." Sensors 9(7):5423-45.

Di Fiore, Barbara, Marilena Ciciarello, and Patrizia Lavia. 2004. "Mitotic Functions of the Ran GTPase Network: The Importance of Being in the Right Place at the Right Time." Cell Cycle (Georgetown, Tex.) 3(3):305-13. 
Fischer, Jessica, Roman Teimer, Stefan Amlacher, Ruth Kunze, and Ed Hurt. 2015. "Linker Nups Connect the Nuclear Pore Complex Inner Ring with the Outer Ring and Transport Channel." Nature Structural and Molecular Biology 22(10):774-81.

Fischer, Utz, Jochen Huber, Wilbert C. Boelens, Lain W. Mattajt, and Reinhard Lührmann. 1995. "The HIV-1 Rev Activation Domain Is a Nuclear Export Signal That Accesses an Export Pathway Used by Specific Cellular RNAs." Cell 82(3):475-83.

Fleta-Soriano, Eric, Javier P. Martinez, Bettina Hinkelmann, Klaus Gerth, Peter Washausen, Juana Diez, Ronald Frank, Florenz Sasse, and Andreas Meyerhans. 2014. "The Myxobacterial Metabolite Ratjadone A Inhibits HIV Infection by Blocking the Rev/CRM1-Mediated Nuclear Export Pathway." Microbial Cell Factories 13(1):17.

Fontoura, Beatriz M. A., Paula A. Faria, and Daniel R. Nussenzveig. 2005. "Viral Interactions with the Nuclear Transport Machinery: Discovering and Disrupting Pathways." IUBMB Life 57(2):65-72.

Foo, Roger S. Y., Young Jae Nam, Marc Jason Ostreicher, Mark D. Metzl, Russell S. Whelan, Chang Fu Peng, Anthony W. Ashton, Weimin Fu, Kartik Mani, Suet Feung Chin, Elena Provenzano, Ian Ellis, Nichola Figg, Sarah Pinder, Martin R. Bennett, Carlos Caldas, and Richard N. Kitsis. 2007.

"Regulation of P53 Tetramerization and Nuclear Export by ARC." Proceedings of the National Academy of Sciences of the United States of America 104(52):20826-31.

Fornerod, Maarten, Jan Van Deursen, Sjozef Van Baal, Albert Reynolds, Donna Davis, K. Gopal Murti, Jack Fransen, and Gerard Grosveld. 1997. "The Human Homologue of Yeast CRM1 Is in a Dynamic Subcomplex with CAN/Nup214 and a Novel Nuclear Pore Component Nup88." EMBO Journal 16(4):807-16.

Fornerod, Maarten, Mutsuhito Ohno, Minoru Yoshida, and lain W. Mattaj. 1997. "CRM1 Is an Export Receptor for Leucine-Rich Nuclear Export Signals." Cell 90(6):1051-60.

Fukuda, Makoto, Shiro Asano, Takahiro Nakamura, Makoto Adachi, Minoru Yoshida, Mitsuhiro Yanagida, and Eisuke Nishida. 1997. "CRM1 Is Responsible for Intracellular Transport Mediated by the Nuclear Export Signal." Nature 390(6657):308-11.

Fung, Ho Yee Joyce, Szu Chin Fu, Chad A. Brautigam, and Yuh Min Chook. 2015. "Structural Determinants of Nuclear Export Signal Orientation in Binding to Exportin CRM1." ELife 4(September 2015):1-19.

Fung, Ho Yee Joyce, Szu Chin Fu, and Yuh Min Chook. 2017. "Nuclear Export Receptor CRM1 Recognizes Diverse Conformations in Nuclear Export Signals." ELife 6:1-13.

Gandhi, Ujjawal H., William Senapedis, Erkan Baloglu, Thaddeus J. Unger, Ajai Chari, Dan Vogl, and Robert F. Cornell. 2018. "Clinical Implications of Targeting XPO1-Mediated Nuclear Export in Multiple Myeloma." Clinical Lymphoma, Myeloma and Leukemia 18(5):335-45.

Garzon, Ramiro, Michael Savona, Rachid Baz, Michael Andreeff, Nashat Gabrail, Martin Gutierrez, Lynn Savoie, Paul Morten Mau-Sorensen, Nina Wagner-Johnston, Karen Yee, Thaddeus J. Unger, Jean Richard Saint-Martin, Robert Carlson, Tami Rashal, Trinayan Kashyap, Boris Klebanov, Sharon Shacham, Michael Kauffman, and Richard Stone. 2017. "A Phase 1 Clinical Trial of Single-Agent Selinexor in Acute Myeloid Leukemia." Blood 129(24):3165-74.

Gasiorowski, Joshua Z. and David A. Dean. 2003. "Mechanisms of Nuclear Transport and Interventions." Advanced Drug Delivery Reviews 55(6):703-16. 
Getz, Elise Burmeister, Ming Xiao, Tania Chakrabarty, Roger Cooke, and Paul R. Selvin. 1999. “A Comparison between the Sulfhydryl Reductants Tris(2- Carboxyethyl)Phosphine and Dithiothreitol for Use in Protein Biochemistry." Analytical Biochemistry 273(1):73-80.

Ghildyal, Reena, Adeline Ho, Manisha Dias, Lydia Soegiyono, Phillip G. Bardin, Kim C. Tran, Michael N. Teng, and David A. Jans. 2009. "The Respiratory Syncytial Virus Matrix Protein Possesses a Crm1Mediated Nuclear Export Mechanism." Journal of Virology 83(11):5353-62.

Görlich, D. and I. W. Mattaj. 1996. "Nucleocytoplasmic Transport." Science (New York, N.Y.) 271(5255):1513-18.

Görlich, D., N. Panté, U. Kutay, U. Aebi, and F. R. Bischoff. 1996. "Identification of Different Roles for RanGDP and RanGTP in Nuclear Protein Import." The EMBO Journal 15(20):5584-94.

Görlich, Dirk, Marylena Dabrowski, F. Ralf Bischoff, Ulrike Kutay, Peer Bork, Enno Hartmann, Siegfried Prehn, and Elisa Izaurralde. 1997. "A Novel Class of RanGTP Binding Proteins." Journal of Cell Biology 138(1):65-80.

Görlich, Dirk and Ulrike Kutay. 1999. "Transport Between the Cell Nucleus and the Cytoplasm." Annual Review of Cell and Developmental Biology 15(1):607-60.

Grant, Richard P., David Neuhaus, and Murray Stewart. 2003. "Structural Basis for the Interaction between the Tap/NXF1 UBA Domain and FG Nucleoporins at 1A Resolution." Journal of Molecular Biology 326(3):849-58.

Gravina, Giovanni Luca, William Senapedis, Dilara McCauley, Erkan Baloglu, Sharon Shacham, and Claudio Festuccia. 2014. "Nucleo-Cytoplasmic Transport as a Therapeutic Target of Cancer." Journal of Hematology and Oncology 7(1).

Gravina, Giovanni, Monica Tortoreto, Andrea Mancini, Alessandro Addis, Ernesto Di Cesare, Andrea Lenzi, Yosef Landesman, Dilara McCauley, Michael Kauffman, Sharon Shacham, Nadia Zaffaroni, and Claudio Festuccia. 2014. "XPO1/CRM1-Selective Inhibitors of Nuclear Export (SINE) Reduce Tumor Spreading and Improve Overall Survival in Preclinical Models of Prostate Cancer (PCa)." Journal of Hematology \& Oncology 7(1):46.

Güttler, Thomas and Dirk Görlich. 2011. "Ran-Dependent Nuclear Export Mediators: A Structural Perspective." EMBO Journal 30(17):3457-74.

Güttler, Thomas, Tobias Madl, Piotr Neumann, Danilo Deichsel, Lorenzo Corsini, Thomas Monecke, Ralf Ficner, Michael Sattler, and Dirk Görlich. 2010. "NES Consensus Redefined by Structures of PKIType and Rev-Type Nuclear Export Signals Bound to CRM1." Nature Structural \& Molecular Biology 17(11):1367-76.

Gwack, Yousang, Sonia Sharma, Julie Nardone, Bogdan Tanasa, Alina luga, Sonal Srikanth, Heidi Okamura, Diana Bolton, Stefan Feske, Patrick G. Hogan, and Anjana Rao. 2006. "A Genome-Wide Drosophila RNAi Screen Identifies DYRK-Family Kinases as Regulators of NFAT." Nature 441(7093):646-50.

Haines, Jeffery D., Olivier Herbin, Belén De La Hera, Oscar G. Vidaurre, Gregory A. Moy, Qingxiang Sun, Ho Yee Joyce Fung, Stefanie Albrecht, Konstantina Alexandropoulos, Dilara McCauley, Yuh Min Chook, Tanja Kuhlmann, Grahame J. Kidd, Sharon Shacham, and Patrizia Casaccia. 2015. "Nuclear Export Inhibitors Avert Progression in Preclinical Models of Inflammatory Demyelination." Nature Neuroscience 18(4):511-20. 
Hamamoto, Tetsuo, Shigemichi Gunji, Hiroaki Tsuji, and Teruhiko Beppu. 1983. "Leptomycins a and b, New Antifungal Antibiotics I. Taxonomy of the Producing Strain and Their Fermentation, Purification and Characterization." The Journal of Antibiotics 36(6):639-45.

Hamamoto, Tetsuo, Takeshi Uozumi, and Teruhiko Beppu. 1985. "Leptomycins A and B, New Antifungal Antibiotics III. Mode of Action of Leptomycin on Schizosaccharomyces Pombe." The Journal of Antibiotics 38(11):1573-80.

Harel, Amnon and Douglass J. Forbes. 2004. "Importin Beta: Conducting a Much Larger Cellular Symphony." Molecular Cell 16(3):319-30.

Hayakawa, Yoichi, Kin Ya Sohda, Kazuo Shin-Ya, Tomomi Hidaka, and Haruo Seto. 1995. "Anguinomycins C and D, New Antitumor Antibiotics with Selective Cytotoxicity against Transformed Cells." The Journal of Antibiotics 48(9):954-61.

Henderson, Beric R. 2000. "Nuclear-Cytoplasmic Shuttling of APC Regulates $\beta$-Catenin Subcellular Localization and Turnover." Nature Cell Biology 2(9):653-60.

Henderson, Beric R. and Francois Fagotto. 2002. "The Ins and Outs of APC and Beta-Catenin Nuclear Transport." EMBO Reports 3(9):834-39.

Hill, Richard, Bastien Cautain, Nuria De Pedro, and Wolfgang Link. 2014. "Targeting Nucleocytoplasmic Transport in Cancer Therapy." Oncotarget 5(1):11-28.

Hing, Z. A., H. Y. J. Fung, P. Ranganathan, S. Mitchell, D. El-Gamal, J. A. Woyach, K. Williams, V. M. Goettl, J. Smith, X. Yu, X. Meng, Q. Sun, T. Cagatay, A. M. Lehman, D. M. Lucas, E. Baloglu, S. Shacham, M. G. Kauffman, J. C. Byrd, Y. M. Chook, R. Garzon, and R. Lapalombella. 2016. "Next-Generation XPO1 Inhibitor Shows Improved Efficacy and in Vivo Tolerability in Hematological Malignancies." Leukemia.

Hintersteiner, Martin, Géza Ambrus, Janna Bednenko, Mario Schmied, Andrew J. S. Knox, Nicole Claudia Meisner, Hubert Gstach, Jan Marcus Seifert, Eric L. Singer, Larry Gerace, and Manfred Auer. 2010. "Identification of a Small Molecule Inhibitor of Importin $\beta$ Mediated Nuclear Import by Confocal On-Bead Screening of Tagged One-Bead One-Compound Libraries." ACS Chemical Biology 5(10):967-79.

Ho, Jennifer Hei Ngam, George Kallstrom, and Arlen W. Johnson. 2000. "Nmd3p Is a Crm1p-Dependent Adapter Protein for Nuclear Export of the Large Ribosomal Subunit." Journal of Cell Biology 151(5):1057-66.

Hoelz, André, Erik W. Debler, and Günter Blobel. 2011. "The Structure of the Nuclear Pore Complex." Annual Review of Biochemistry 80(1):613-43.

Hollstein, Monica, David Sidransky, Bert Vogelstein, and Curtis C. Harris. 1991. "P53 Mutations in Human Cancers." Science 253(5015):49-53.

Howell, Jessica J. and Markus Stoffel. 2009. "Nuclear Export-Independent Inhibition of Foxa2 by Insulin." Journal of Biological Chemistry 284(37):24816-24.

Huang, Wei Yi, Lu Yue, Wen Shen Qiu, Li Wei Wang, Xiao Han Zhou, and Yuan Jue Sun. 2009. "Prognostic Value of CRM1in Pancreas Cancer." Clinical and Investigative Medicine 32(6).

Huber, Jochen, Udo Cronshagen, Michinori Kadokura, Christopher Marshallsay, Takeshi Wada, Mitsuo 
Sekine, and Reinhard Lührmann. 1998. "Snurportin1, an M3G-Cap-Specific Nuclear Import Receptor with a Novel Domain Structure." EMBO Journal 17(14):4114-26.

Hughes, J. P., S. S. Rees, S. B. Kalindjian, and K. L. Philpott. 2011. "Principles of Early Drug Discovery." British Journal of Pharmacology 162(6):1239-49.

Hwang, Chae Young, Ick Young Kim, and Ki Sun Kwon. 2007. "Cytoplasmic Localization and Ubiquitination of P21Cip1 by Reactive Oxygen Species." Biochemical and Biophysical Research Communications 358(1):219-25.

Inoue, Hiromi, Michael Kauffman, Sharon Shacham, Yosef Landesman, Joy Yang, Christopher P. Evans, and Robert H. Weiss. 2013. "CRM1 Blockade by Selective Inhibitors of Nuclear Export Attenuates Kidney Cancer Growth." Journal of Urology 189(6):2317-26.

Isgro, Timothy A. and Klaus Schulten. 2005. "Binding Dynamics of Isolated Nucleoporin Repeat Regions to Importin- $\beta$." Structure 13(12):1869-79.

Ishida, Noriko, Taichi Hara, Takumi Kamura, Minoru Yoshida, Keiko Nakayama, and Keiichi I. Nakayama. 2002. "Phosphorylation of P27Kip1 on Serine 10 Is Required for Its Binding to CRM1 and Nuclear Export." Journal of Biological Chemistry 277(17):14355-58.

Jamieson, Cara, Manisha Sharma, and Beric R. Henderson. 2014. "Targeting the $\beta$-Catenin Nuclear Transport Pathway in Cancer." Seminars in Cancer Biology 27:20-29.

Jeyasekharan, Anand D., Yang Liu, Hiroyoshi Hattori, Venkat Pisupati, Asta Bjork Jonsdottir, Eeson Rajendra, Miyoung Lee, Elayanambi Sundaramoorthy, Simon Schlachter, Clemens F. Kaminski, Yaara Ofir-Rosenfeld, Ko Sato, Jane Savill, Nabieh Ayoub, and Ashok R. Venkitaraman. 2013. “A Cancer-Associated BRCA2 Mutation Reveals Masked Nuclear Export Signals Controlling Localization." Nature Structural and Molecular Biology 20(10):1191-1201.

Jiao, W., H. M. Lin, J. Datta, T. Braunschweig, J. Y. Chung, S. M. Hewitt, and S. G. Rane. 2008. “Aberrant Nucleocytoplasmic Localization of the Retinoblastoma Tumor Suppressor Protein in Human Cancer Correlates with Moderate/Poor Tumor Differentiation." Oncogene 27(22):3156-64.

Jiao, Wan, Jashodeep Datta, Huei Min Lin, Miroslav Dundr, and Sushil G. Rane. 2006. "Nucleocytoplasmic Shuttling of the Retinoblastoma Tumor Suppressor Protein via Cdk Phosphorylation-Dependent Nuclear Export." Journal of Biological Chemistry 281(49):38098-108.

Kakisaka, Michinori, Takafumi Mano, and Yoko Aida. 2016. “A High-Throughput Screening System Targeting the Nuclear Export Pathway via the Third Nuclear Export Signal of Influenza A Virus Nucleoprotein." Virus Research 217:23-31.

Kalab, Petr, Karsten Weis, and Rebecca Heald. 2002. "Visualization of a Ran-GTP Gradient in Interphase and Mitotic Xenopus Egg Extracts." Science 295(5564):2452-56.

Kalesse, M., M. Christmann, U. Bhatt, M. Quitschalle, E. Claus, A. Saeed, A. Burzlaff, C. Kasper, L. O. Haustedt, E. Hofer, T. Scheper, and W. Beil. 2001. "The Chemistry and Biology of Ratjadone." Chembiochem : A European Journal of Chemical Biology 2(9):709-14.

Kalid, Ori, Dora Toledo Warshaviak, Sharon Shechter, Woody Sherman, and Sharon Shacham. 2012. "Consensus Induced Fit Docking (CIFD): Methodology, Validation, and Application to the Discovery of Novel Crm1 Inhibitors." Journal of Computer-Aided Molecular Design 26(11):1217-28. 
Kehlenbach, Ralph H., Achim Dickmanns, and Larry Gerace. 1998. "Nucleocytoplasmic Shuttling Factors Including Ran and CRM1 Mediate Nuclear Export of NFAT in Vitro." Journal of Cell Biology 141(4):863-74.

Kehlenbach, Ralph H., Achim Dickmanns, Angelika Kehlenbach, Tinglu Guan, and Larry Gerace. 1999. “A Role for RanBP1 in the Release of CRM1 from the Nuclear Pore Complex in a Terminal Step of Nuclear Export." Journal of Cell Biology 145(4):645-57.

Kırlı, Koray, Samir Karaca, Heinz Jürgen Dehne, Matthias Samwer, Kuan Ting Pan, Christof Lenz, Henning Urlaub, and Dirk Görlich. 2015. "A Deep Proteomics Perspective on CRM1-Mediated Nuclear Export and Nucleocytoplasmic Partitioning." ELife 4(DECEMBER2015).

Kojima, Kensuke, Steven M. Kornblau, Vivian Ruvolo, Archana Dilip, Seshagiri Duvvuri, R. Eric Davis, Min Zhang, Zhiqiang Wang, Kevin R. Coombes, Nianxiang Zhang, Yi Hua Qiu, Jared K. Burks, Hagop Kantarjian, Sharon Shacham, Michael Kauffman, and Michael Andreeff. 2013. "Prognostic Impact and Targeting of CRM1 in Acute Myeloid Leukemia." Blood 121(20):4166-74.

Kontoyianni, Maria. 2017. "Docking and Virtual Screening in Drug Discovery." Pp. 255-66 in Methods in Molecular Biology. Vol. 1647. Humana Press Inc.

Köster, Mario, Søren Lykke-Andersen, Yasser A. Elnakady, Klaus Gerth, Peter Washausen, Gerhard Höfle, Florenz Sasse, Jørgen Kjems, and Hansjörg Hauser. 2003. "Ratjadones Inhibit Nuclear Export by Blocking CRM1/Exportin 1." Experimental Cell Research 286(2):321-31.

Kosugi, Shunichi, Masako Hasebe, Masaru Tomita, and Hiroshi Yanagawa. 2008. "Nuclear Export Signal Consensus Sequences Defined Using a Localization-Based Yeast Selection System." Traffic 9(12):2053-62.

Kowalik, Artur, Janusz Kopczyński, Elżbieta Wypiórkiewicz, Stanisław Góźdź, Ryszard Meżyk, and Janusz Aleksander Siedlecki. 2013. "Active Transport of RB Protein from the Nucleus to the Cytoplasm as One of the Development Mechanisms of HER2-Positive Breast Cancer." Polish Journal of Pathology : Official Journal of the Polish Society of Pathologists 64(1):9-14.

Koyama, Masako, Hidemi Hirano, Natsuki Shirai, and Yoshiyuki Matsuura. 2017. "Crystal Structure of the Xpo1p Nuclear Export Complex Bound to the SxFG/PxFG Repeats of the Nucleoporin Nup42p." Genes to Cells 22(10):861-75.

Koyama, Masako and Yoshiyuki Matsuura. 2010. "An Allosteric Mechanism to Displace Nuclear Export Cargo from CRM1 and RanGTP by RanBP1." EMBO Journal.

Kudo, N., N. Matsumori, H. Taoka, D. Fujiwara, E. P. Schreiner, B. Wolff, M. Yoshida, and S. Horinouchi. 1999. "Leptomycin B Inactivates CRM1/Exportin 1 by Covalent Modification at a Cysteine Residue in the Central Conserved Region." Proceedings of the National Academy of Sciences.

Kudo, Nobuaki, Barbara Wolff, Toshihiro Sekimoto, Erwin P. Schreiner, Yoshihiro Yoneda, Mitsuhiro Yanagida, Sueharu Horinouchi, and Minoru Yoshida. 1998. "Leptomycin B Inhibition of SignalMediated Nuclear Export by Direct Binding to CRM1." Experimental Cell Research 242(2):540-47.

Kutay, U., S. Guttinger, and D. Gorlich. 2005. "Leucine-Rich Nuclear-Export Signals: Born to Be Weak\rTransport between the Cell Nucleus and the Cytoplasm." Trends Cell Biol 15(3):121-24.

Lange, Allison, Ryan E. Mills, Christopher J. Lange, Murray Stewart, Scott E. Devine, and Anita H. Corbett. 2007. "Classical Nuclear Localization Signals: Definition, Function, and Interaction with Importin $\alpha$. " 
Journal of Biological Chemistry 282(8):5101-5.

Lapalombella, Rosa, Qingxiang Sun, Katie Williams, Larissa Tangeman, Shruti Jha, Yiming Zhong, Virginia Goettl, Emilia Mahoney, Caroline Berglund, Sneha Gupta, Alicia Farmer, Rajeswaran Mani, Amy J. Johnson, David Lucas, Xiaokui Mo, Dirk Daelemans, Vincent Sandanayaka, Sharon Shechter, Dilara McCauley, Sharon Shacham, Michael Kauffman, Yuh Min Chook, and John C. Byrd. 2012. "Selective Inhibitors of Nuclear Export Show That CRM1/XPO1 Is a Target in Chronic Lymphocytic Leukemia." Blood 120(23):4621-34.

De Laté, Perle Latré, Aurélie Pépin, Hind Assaf-Vandecasteele, Christophe Espinasse, Valérie Nicolas, Marie Liesse Asselin-Labat, Jacques Bertoglio, Marc Pallardy, and Armelle Biola-Vidamment. 2010. "Glucocorticoid-Induced Leucine Zipper (GILZ) Promotes the Nuclear Exclusion of FOXO3 in a Crm1Dependent Manner." Journal of Biological Chemistry 285(8):5594-5605.

Lee, Yoonji, Jimin Pei, Jordan M. Baumhardt, Yuh Min Chook, and Nick V. Grishin. 2019. "Structural Prerequisites for CRM1-Dependent Nuclear Export Signaling Peptides: Accessibility, Adapting Conformation, and the Stability at the Binding Site." Scientific Reports 9(1):1-13.

Leopold, W. R., J. L. Shillis, A. E. Mertus, J. M. Nelson, B. J. Roberts, and R. C. Jackson. 1984. "Anticancer Activity of the Structurally Novel Antibiotic Cl-920 and Its Analogues." Cancer Research 44(5):192832.

Levine, Arnold J. and Benjamin Greenbaum. 2012. "The Maintenance of Epigenetic States by P53: The Guardian of the Epigenome." Oncotarget 3(12):1503-4.

Lin, Daniel H., Ana R. Correia, Sarah W. Cai, Ferdinand M. Huber, Claudia A. Jette, and André Hoelz. 2018. "Structural and Functional Analysis of MRNA Export Regulation by the Nuclear Pore Complex." Nature Communications 9(1):1-19.

Lin, Daniel H. and André Hoelz. 2019. "The Structure of the Nuclear Pore Complex (An Update)." Annual Review of Biochemistry 88(1):725-83.

Lin, Daniel H., Tobias Stuwe, Sandra Schilbach, Emily J. Rundlet, Thibaud Perriches, George Mobbs, Yanbin Fan, Karsten Thierbach, Ferdinand M. Huber, Leslie N. Collins, Andrew M. Davenport, Young E. Jeon, and André Hoelz. 2016. "Architecture of the Symmetric Core of the Nuclear Pore." Science 352(6283):aaf1015.

Lionta, Evanthia, George Spyrou, Demetrios Vassilatis, and Zoe Cournia. 2014. "Structure-Based Virtual Screening for Drug Discovery: Principles, Applications and Recent Advances." Current Topics in Medicinal Chemistry 14(16):1923-38.

Liu, Sai Man and Murray Stewart. 2005. "Structural Basis for the High-Affinity Binding of Nucleoporin Nup1p to the Saccharomyces Cerevisiae Importin- $\beta$ Homologue, Kap95p." Journal of Molecular Biology 349(3):515-25.

Lohrum, M. A., D. B. Woods, R. L. Ludwig, E. Bálint, and K. H. Vousden. 2001. "C-Terminal Ubiquitination of P53 Contributes to Nuclear Export." Molecular and Cellular Biology 21(24):8521-32.

Lu, Weng, Radhika Pochampally, Lihong Chen, Mullika Traidej, Yiliang Wang, and Jiandong Chen. 2000. "Nuclear Exclusion of P53 in a Subset of Tumors Requires MDM2 Function." Oncogene 19(2):23240.

Mahmoud, Abeer M., Virgilia Macias, Umaima Al-alem, Ryan J. Deaton, Andre Kadjaksy-Balla, Peter H. 
Gann, and Garth H. Rauscher. 2017. "BRCA1 Protein Expression and Subcellular Localization in Primary Breast Cancer: Automated Digital Microscopy Analysis of Tissue Microarrays" edited by R. Samant. PLOS ONE 12(9):e0184385.

Makde, Ravindra D., Joseph R. England, Hemant P. Yennawar, and Song Tan. 2010. "Structure of RCC1 Chromatin Factor Bound to the Nucleosome Core Particle." Nature 467(7315):562-66.

Mancini, Maria and Alex Toker. 2009. "NFAT Proteins: Emerging Roles in Cancer Progression." Nature Reviews Cancer 9(11):810-20.

Mathew, Cynthia and Reena Ghildyal. 2017. "CRM1 Inhibitors for Antiviral Therapy." Frontiers in Microbiology.

Matsuura, Yoshiyuki and Murray Stewart. 2005. "Nup50/Npap60 Function in Nuclear Protein Import Complex Disassembly and Importin Recycling." EMBO Journal 24(21):3681-89.

Mittnacht, S. 1998. "Control of PRB Phosphorylation." Current Opinion in Genetics \& Development $8(1): 21-27$.

Mohr, Dagmar, Steffen Frey, Torsten Fischer, Thomas Güttler, and Dirk Görlich. 2009. "Characterisation of the Passive Permeability Barrier of Nuclear Pore Complexes." EMBO Journal 28(17):2541-53.

Monecke, Thomas, Achim Dickmanns, and Ralf Ficner. 2014. "Allosteric Control of the Exportin CRM1 Unraveled by Crystal Structure Analysis." FEBS Journal 281(18):4179-94.

Monecke, Thomas, Thomas Güttler, Piotr Neumann, Achim Dickmanns, Dirk Görlich, and Ralf Ficner. 2009. "Crystal Structure of the Nuclear Export Receptor CRM1 in Complex with Snurportin1 and RanGTP." Science 324(5930):1087-91.

Monecke, Thomas, David Haselbach, Béla Voß, Andreas Russek, Piotr Neumann, Emma Thomson, Ed Hurt, Ulrich Zachariae, Holger Stark, Helmut Grubmuïler, Achim Dickmanns, Ralf Ficner.2013. "Structural Basis for Cooperativity of CRM1 Export Complex Formation." Proceedings of the National Academy of Sciences of the United States of America 110(3):960-65.

Müller, Martin R. and Anjana Rao. 2010. "NFAT, Immunity and Cancer: A Transcription Factor Comes of Age." Nature Reviews Immunology 10(9):645-56.

Murakami, Nobutoshi, Ying Ye, Motoyuki Kawanishi, Shunji Aoki, Nobuaki Kudo, Minoru Yoshida, Emi E. Nakayama, Tatsuo Shioda, and Motomasa Kobayashi. 2002. "New Rev-Transport Inhibitor with Anti-HIV Activity from Valerianae Radix." Bioorganic \& Medicinal Chemistry Letters 12(20):280710.

Mutka, Sarah C., Wen Qing Yang, Steven D. Dong, Shannon L. Ward, Darren A. Craig, Pieter B. M. W. M. Timmermans, and Sumati Murli. 2009. "Identification of Nuclear Export Inhibitors with Potent Anticancer Activity in Vivo." Cancer Research 69(2):510-17.

Najera, Isabel, Marion Krieg, and Jonathan Karn. 1999. "Synergistic Stimulation of HIV-1 Rev-Dependent Export of Unspliced MRNA to the Cytoplasm by HnRNP A1." Journal of Molecular Biology 285(5):1951-64.

Nakano, Kazumi and Toshiki Watanabe. 2016. "HTLV-1 Rex Tunes the Cellular Environment Favorable for Viral Replication." Viruses 8(3):58.

Neufeld, K L, F. Zhang, B. R. Cullen, and R. L. White. 2000. “APC-Mediated Downregulation of Beta- 
Catenin Activity Involves Nuclear Sequestration and Nuclear Export." EMBO Reports 1(6):519-23.

Neufeld, Kristi L., David A. Nix, Hal Bogerd, Yibin Kang, Mary C. Beckerle, Bryan R. Cullen, and Raymond L. White. 2000. "Adenomatous Polyposis Coli Protein Contains Two Nuclear Export Signals and Shuttles between the Nucleus and Cytoplasm." Proceedings of the National Academy of Sciences of the United States of America 97(22):12085-90.

Neufeld, Kristi L. and Raymond L. White. 1997. "Nuclear and Cytoplasmic Localizations of the Adenomatous Polyposis Coli Protein." Proceedings of the National Academy of Sciences of the United States of America 94(7):3034-39.

Newlands, E. S., G. J. Rustin, and M. H. Brampton. 1996. "Phase I Trial of Elactocin.” British Journal of Cancer 74(4):648-49.

Nishi, K., M. Yoshida, D. Fujiwara, M. Nishikawa, S. Horinouchi, and T. Beppu. 1994. "Leptomycin B Targets a Regulatory Cascade of Crm1, a Fission Yeast Nuclear Protein, Involved in Control of Higher Order Chromosome Structure and Gene Expression." The Journal of Biological Chemistry 269(9):6320-24.

Nitiss, John L. 2009a. "DNA Topoisomerase II and Its Growing Repertoire of Biological Functions." Nature Reviews Cancer 9(5):327-37.

Nitiss, John L. 2009b. "Targeting DNA Topoisomerase II in Cancer Chemotherapy." Nature Reviews Cancer 9(5):338-50.

Niu, Mingshan, Sijin Wu, Lei Mao, and Yongliang Yang. 2013. "CRM1 Is a Cellular Target of Curcumin: New Insights for the Myriad of Biological Effects of an Ancient Spice." Traffic 14(10):1042-52.

Niu, Mingshan, Xiaoyu Xu, Yangling Shen, Yao Yao, Jianlin Qiao, Feng Zhu, Lingyu Zeng, Xuejiao Liu, and Kailin Xu. 2015. "Piperlongumine Is a Novel Nuclear Export Inhibitor with Potent Anticancer Activity." Chemico-Biological Interactions 237:66-72.

Noske, Aurelia, Wilko Weichert, Silvia Niesporek, Annika Röske, Ann Christin Buckendahl, Ines Koch, Jalid Sehouli, Manfred Dietel, and Carsten Denkert. 2008. "Expression of the Nuclear Export Protein Chromosomal Region Maintenance/Exportin 1/Xpo1 Is a Prognostic Factor in Human Ovarian Cancer." Cancer 112(8):1733-43.

Ohno, Mutsuhito, Alexandra Segref, Angela Bachi, Matthias Wilm, and lain W. Mattaj. 2000. "PHAX, a Mediator of U SnRNA Nuclear Export Whose Activity Is Regulated by Phosphorylation." Cell 101(2):187-98.

Ossareh-Nazari, Batool, Françoise Bachelerie, and Catherine Dargemont. 1997. "Evidence for a Role of CRM1 in Signal-Mediated Nuclear Protein Export." Science 278(5335):141-44.

Pan, M. G., Y. Xiong, and F. Chen. 2013. "NFAT Gene Family in Inflammation and Cancer." Current Molecular Medicine 13(4):543-54.

Paragas, J., J. Talon, R. E. O’Neill, D. K. Anderson, A. García-Sastre, and P. Palese. 2001. “Influenza B and C Virus NEP (NS2) Proteins Possess Nuclear Export Activities." Journal of Virology 75(16):7375-83.

Paraskeva, Efrosyni, Elisa Izaurralde, F. Ralf Bischoff, Jochen Huber, Ulrike Kutay, Enno Hartmann, Reinhard Lührmann, and Dirk Görlich. 2000. "CRM1-Mediated Recycling of Snurportin 1 to the Cytoplasm." Journal of Cell Biology 145(2):255-64. 
Pasdeloup, David, Nicolas Poisson, Hélène Raux, Yves Gaudin, Rob W. H. Ruigrok, and Danielle Blondel. 2005. "Nucleocytoplasmic Shuttling of the Rabies Virus P Protein Requires a Nuclear Localization Signal and a CRM1-Dependent Nuclear Export Signal." Virology 334(2):284-93.

Patel, Disha, Joseph D. Bauman, and Eddy Arnold. 2014. "Advantages of Crystallographic Fragment Screening: Functional and Mechanistic Insights from a Powerful Platform for Efficient Drug Discovery." Progress in Biophysics and Molecular Biology 116(2-3):92-100.

Pathria, Gaurav, Christine Wagner, and Stephan N. Wagner. 2012. "Inhibition of CRM1-Mediated Nucleocytoplasmic Transport: Triggering Human Melanoma Cell Apoptosis by Perturbing Multiple Cellular Pathways." Journal of Investigative Dermatology 132(12):2780-90.

Perwitasari, Olivia, Scott Johnson, Xiuzhen Yan, Elizabeth Howerth, Sharon Shacham, Yosef Landesman, Erkan Baloglu, Dilara McCauley, Sharon Tamir, S. Mark Tompkins, and Ralph A. Tripp. 2014. "Verdinexor, a Novel Selective Inhibitor of Nuclear Export, Reduces Influenza a Virus Replication in Vitro and in Vivo." Journal of Virology 88(17):10228-43.

Peters, Reiner. 2006. "Introduction to Nucleocytoplasmic Transport: Molecules and Mechanisms." Methods in Molecular Biology (Clifton, N.J.) 322:235-58.

Petosa, Carlo, Guy Schoehn, Peter Askjaer, Ulrike Bauer, Martine Moulin, Ulrich Steuerwald, Montserrat Soler-López, Florence Baudin, lain W. Mattaj, and Christoph W. Müller. 2004. "Architecture of CRM1/Exportin1 Suggests How Cooperativity Is Achieved during Formation of a Nuclear Export Complex." Molecular Cell 16(5):761-75.

Port, Sarah A., Thomas Monecke, Achim Dickmanns, Christiane Spillner, Romina Hofele, Henning Urlaub, Ralf Ficner, and Ralph H. Kehlenbach. 2015. "Structural and Functional Characterization of CRM1Nup214 Interactions Reveals Multiple FG-Binding Sites Involved in Nuclear Export." Cell Reports 13(4):690-702.

Powell, Steven M., Nathan Zilz, Yasmin Beazer-Barclay, Tracy M. Bryan, Stanley R. Hamilton, Stephen N. Thibodeau, Bert Vogelstein, and Kenneth W. Kinzler. 1992. "APC Mutations Occur Early during Colorectal Tumorigenesis." Nature 359(6392):235-37.

Pumroy, Ruth A., Jonathan D. Nardozzi, Darren J. Hart, Michael J. Root, and Gino Cingolani. 2012. "Nucleoporin Nup50 Stabilizes Closed Conformation of Armadillo Repeat 10 in Importin A5." Journal of Biological Chemistry 287(3):2022-31.

Qin, Jiang Jiang, Subhasree Nag, Wei Wang, Jianwei Zhou, Wei Dong Zhang, Hui Wang, and Ruiwen Zhang. 2014. "NFAT as Cancer Target: Mission Possible?" Biochimica et Biophysica Acta - Reviews on Cancer 1846(2):297-311.

Rao, Anjana, Chun Luo, and Patrick G. Hogan. 1997. "TRANSCRIPTION FACTORS OF THE NFAT FAMILY:Regulation and Function." Annual Review of Immunology 15(1):707-47.

Rees, David C., Miles Congreve, Christopher W. Murray, and Robin Carr. 2004. "Fragment-Based Lead Discovery." Nature Reviews. Drug Discovery 3(8):660-72.

Rexach, Michael and Günter Blobel. 1995. "Protein Import into Nuclei: Association and Dissociation Reactions Involving Transport Substrate, Transport Factors, and Nucleoporins." Cell 83(5):683-92.

Roberts, B. J., K. L. Hamelehle, J. S. Sebolt, and W. R. Leopold. 1986. "In Vivo and in Vitro Anticancer Activity of the Structurally Novel and Highly Potent Antibiotic Cl-940 and Its Hydroxy Analog (PD 
114,721)." Cancer Chemotherapy and Pharmacology 16(2):95-101.

Rodríguez, Jose A. and Beric R. Henderson. 2000. "Identification of a Functional Nuclear Export Sequence in BRCA1." Journal of Biological Chemistry 275(49):38589-96.

Rodriguez, José Antonio, Stefan Schüchner, Wendy W. Y. Au, Megan Fabbro, and Beric R. Henderson. 2004. "Nuclear-Cytoplasmic Shuttling of BARD1 Contributes to Its Proapoptotic Activity and Is Regulated by Dimerization with BRCA1." Oncogene 23(10):1809-20.

Saito, N., K. Sakakibara, T. Sato, J. M. Friedman, D. W. Kufe, D. D. VonHoff, and T. Kawabe. 2014. "CBS9106-Induced CRM1 Degradation Is Mediated by Cullin Ring Ligase Activity and the Neddylation Pathway." Molecular Cancer Therapeutics 13(12):3013-23.

Sakakibara, Keiichi, Naoya Saito, Takuji Sato, Atsushi Suzuki, Yoko Hasegawa, Jonathan M. Friedman, Donald W. Kufe, Daniel D. VonHoff, Tadahiko Iwami, and Takumi Kawabe. 2011. "CBS9106 Is a Novel Reversible Oral CRM1 Inhibitor with CRM1 Degrading Activity." Blood 118(14):3922-31.

Sanchez, Veronica, Jeffrey A. Mahr, Nicole I. Orazio, and Deborah H. Spector. 2007. "Nuclear Export of the Human Cytomegalovirus Tegument Protein Pp65 Requires Cyclin-Dependent Kinase Activity and the Crm1 Exporter." Journal of Virology 81(21):11730-36.

Santiago, Aleixo, Dawei Li, Lisa Y. Zhao, Adam Godsey, and Daiqing Liao. 2013. "P53 SUMOylation Promotes Its Nuclear Export by Facilitating Its Release from the Nuclear Export Receptor CRM1." Molecular Biology of the Cell 24(17):2739-52.

Santivasi, W. L., H. Wang, T. Wang, Q. Yang, X. Mo, E. Brogi, B. G. Haffty, A. B. Chakravarthy, and Fen Xia. 2015. "Association between Cytosolic Expression of BRCA1 and Metastatic Risk in Breast Cancer." British Journal of Cancer 113(3):453-59.

Schmidt, H. Broder and Dirk Görlich. 2016. "Transport Selectivity of Nuclear Pores, Phase Separation, and Membraneless Organelles." Trends in Biochemical Sciences 41(1):46-61.

Schmidt, J., E. Braggio, K. M. Kortuem, J. B. Egan, Y. X. Zhu, C. S. Xin, R. E. Tiedemann, S. E. Palmer, V. M. Garbitt, D. McCauley, M. Kauffman, S. Shacham, M. Chesi, P. L. Bergsagel, and A. K. Stewart. 2013. "Genome-Wide Studies in Multiple Myeloma Identify XPO1/CRM1 as a Critical Target Validated Using the Selective Nuclear Export Inhibitor KPT-276." Leukemia 27(12):2357-65.

Schüchner, Stefan, Varsha Tembe, José A. Rodriguez, and Beric R. Henderson. 2005. "Nuclear Targeting and Cell Cycle Regulatory Function of Human BARD1." Journal of Biological Chemistry 280(10):8855-61.

Scully, R., J. Chen, R. L. Ochs, K. Keegan, M. Hoekstra, J. Feunteun, and D. M. Livingston. 1997. “Dynamic Changes of BRCA1 Subnuclear Location and Phosphorylation State Are Initiated by DNA Damage." Cell 90(3):425-35.

Segref, A., I. W. Mattaj, and M. Ohno. 2001. "The Evolutionarily Conserved Region of the U SnRNA Export Mediator PHAX Is a Novel RNA-Binding Domain That Is Essential for U SnRNA Export." RNA (New York, N.Y.) 7(3):351-60.

Senapedis, William T., Erkan Baloglu, and Yosef Landesman. 2014. "Clinical Translation of Nuclear Export Inhibitors in Cancer." Seminars in Cancer Biology 27:74-86.

Sendino, Maria, Miren Josu Omaetxebarria, and Jose Antonio Rodríguez. 2018. “Hitting a Moving Target: 
Inhibition of the Nuclear Export Receptor XPO1/CRM1 as a Therapeutic Approach in Cancer." Cancer Drug Resistance.

Shen, Aiguo, Yuchan Wang, Yueming Zhao, Lin Zou, Linlin Sun, and Chun Cheng. 2009. "Expression of CRM1 in Human Gliomas and Its Significance in P27 Expression and Clinical Prognosis." Neurosurgery 65(1):153-59.

Sherr, Charles J. and James M. Roberts. 1999. "CDK Inhibitors: Positive and Negative Regulators of G1Phase Progression." Genes and Development 13(12):1501-12.

Smith, Alicia, Amy Brownawell, and lan G. Macara. 1998. "Nuclear Import of Ran Is Mediated by the Transport Factor NTF2." Current Biology 8(25):1403-6.

Smith, Alicia E., Boris M. Slepchenko, James C. Schaff, Leslie M. Loew, and lan G. Macara. 2002. "Systems Analysis of Ran Transport." Science 295(5554):488-91.

Soderholm, Jonathan F., Stephen L. Bird, Petr Kalab, Yasaswini Sampathkumar, Keisuke Hasegawa, Michael Uehara-Bingen, Karsten Weis, and Rebecca Heald. 2011. "Importazole, a Small Molecule

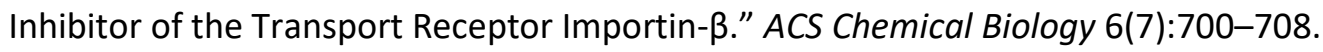

Song, Wei, Ruilian Xie, Aiyu Zhu, Yumei Xu, Yaqin Shi, Yan Shen, Wenwen Zhang, Fang Yang, and Xiaoxiang Guan. 2015. "P27Kip1 and Ser10-Phosphorylated P27Kip1 in Breast Cancer: Clinical Significance and Expression." OncoTargets and Therapy 8:1863-69.

Soniat, Michael and Yuh Min Chook. 2015. "Nuclear Localization Signals for Four Distinct Karyopherin- $\beta$ Nuclear Import Systems." Biochemical Journal 468(3):353-62.

Stade, Katrin, Charleen S. Ford, Christine Guthrie, and Karsten Weis. 1997. "Exportin 1 (Crm1p) Is an Essential Nuclear Export Factor." Cell 90(6):1041-50.

Stommel, J. M., N. D. Marchenko, G. S. Jimenez, U. M. Moll, T. J. Hope, and G. M. Wahl. 1999. “A Leucine-Rich Nuclear Export Signal in the P53 Tetramerization Domain: Regulation of Subcellular Localization and P53 Activity by NES Masking." The EMBO Journal 18(6):1660-72.

Storke, Trond, Bjørn K. Erikstein, Lars Smedshammer, Erik Boye, and Harald B. Steen. 1993. "The Retinoblastoma Gene Product Is Bound in the Nucleus in Early G1 Phase." Experimental Cell Research 204(1):147-55.

Stuwe, Tobias, Christopher J. Bley, Karsten Thierbach, Stefan Petrovic, Sandra Schilbach, Daniel J. Mayo, Thibaud Perriches, Emily J. Rundlet, Young E. Jeon, Leslie N. Collins, Ferdinand M. Huber, Daniel H. Lin, Marcin Paduch, Akiko Koide, Vincent Lu, Jessica Fischer, Ed Hurt, Shohei Koide, Anthony A. Kossiakoff, and André Hoelz. 2015. "Architecture of the Fungal Nuclear Pore Inner Ring Complex." Science 350(6256):56-64.

Suetsugu, Shiro and Tadaomi Takenawa. 2003. "Translocation of N-WASP by Nuclear Localization and Export Signals into the Nucleus Modulates Expression of HSP90." Journal of Biological Chemistry 278(43):42515-23.

Sun, Qingxiang, Yazmin P. Carrasco, Youcai Hu, Xiaofeng Guo, Hamid Mirzaei, John MacMillan, and Yuh Min Chook. 2013. "Nuclear Export Inhibition through Covalent Conjugation and Hydrolysis of Leptomycin B by CRM1." Proceedings of the National Academy of Sciences 110(4):1303-8.

Sun, Qingxiang, Xueqin Chen, Qiao Zhou, Ezra Burstein, Shengyong Yang, and Da Jia. 2016. "Inhibiting 
Cancer Cell Hallmark Features through Nuclear Export Inhibition." Signal Transduction and Targeted Therapy 1.

Syed, Yahiya Y. 2019. "Selinexor: First Global Approval." Drugs.

Tamura, Satoru, Nobuhiro Shimizu, Katsuaki Fujiwara, Masafumi Kaneko, Tominori Kimura, and Nobutoshi Murakami. 2010. "Bioisostere of Valtrate, Anti-HIV Principle by Inhibition for Nuclear Export of Rev." Bioorganic \& Medicinal Chemistry Letters 20(7):2159-62.

Tamura, Satoru, Atsushi Shiomi, Masafumi Kaneko, Ying Ye, Minoru Yoshida, Masayuki Yoshikawa, Tominori Kimura, Motomasa Kobayashi, and Nobutoshi Murakami. 2009. "New Rev-Export Inhibitor from Alpinia Galanga and Structure-Activity Relationship." Bioorganic and Medicinal Chemistry Letters 19(9):2555-57.

Tan, David S. P., Philippe L. Bedard, John Kuruvilla, Lillian L. Siu, and Albiruni R. Abdu. Razak. 2014. "Promising SINEs for Embargoing Nuclear-Cytoplasmic Export as an Anticancer Strategy." Cancer Discovery 4(5):527-37.

Thakur, S., H. B. Zhang, Y. Peng, H. Le, B. Carroll, T. Ward, J. Yao, L. M. Farid, F. J. Couch, R. B. Wilson, and B. L. Weber. 1997. "Localization of BRCA1 and a Splice Variant Identifies the Nuclear Localization Signal." Molecular and Cellular Biology 17(1):444-52.

Thomas, Sherine E., Patrick Collins, Rory Hennell James, Vitor Mendes, Sitthivut Charoensutthivarakul, Chris Radoux, Chris Abell, Anthony G. Coyne, R. Andres Floto, Frank Von Delft, and Tom L. Blundell. 2019. "Structure-Guided Fragmentbased Drug Discovery at the Synchrotron: Screening Binding Sites and Correlations with Hotspot Mapping." Philosophical Transactions of the Royal Society A: Mathematical, Physical and Engineering Sciences 377(2147).

Toda, T., M. Shimanuki, Y. Saka, H. Yamano, Y. Adachi, M. Shirakawa, Y. Kyogoku, and M. Yanagida. 1992. "Fission Yeast Pap1-Dependent Transcription Is Negatively Regulated by an Essential Nuclear Protein, Crm1." Molecular and Cellular Biology 12(12):5474-84.

Tøndel, Kristin, Endre Anderssen, and Finn Drabløs. 2006. "Protein Alpha Shape (PAS) Dock: A New Gaussian-Based Score Function Suitable for Docking in Homology Modelled Protein Structures." Journal of Computer-Aided Molecular Design 20(3):131-44.

Tran, Elizabeth J., Megan C. King, and Anita H. Corbett. 2014. "Macromolecular Transport between the Nucleus and the Cytoplasm: Advances in Mechanism and Emerging Links to Disease." Biochimica et Biophysica Acta - Molecular Cell Research 1843(11):2784-95.

Trott, Oleg and Arthur J. Olson. 2010. "Software News and Update AutoDock Vina: Improving the Speed and Accuracy of Docking with a New Scoring Function, Efficient Optimization, and Multithreading." Journal of Computational Chemistry 31(2):455-61.

Turner, Joel G., Jana Dawson, Michael F. Emmons, Christopher L. Cubitt, Michael Kauffman, Sharon Shacham, Lori A. Hazlehurst, and Daniel M. Sullivan. 2013. "CRM1 Inhibition Sensitizes Drug Resistant Human Myeloma Cells to Topoisomerase II and Proteasome Inhibitors Both in Vitro and Ex Vivo." Journal of Cancer 4(8):614-25.

Turner, Joel G., Jana Dawson, and Daniel M. Sullivan. 2012. "Nuclear Export of Proteins and Drug Resistance in Cancer." Biochemical Pharmacology 83(8):1021-32.

Turner, Joel G., Douglas C. Marchion, Jana L. Dawson, Michael F. Emmons, Lori A. Hazlehurst, Peter 
Washausen, and Daniel M. Sullivan. 2009. "Human Multiple Myeloma Cells Are Sensitized to Topoisomerase II Inhibitors by CRM1 Inhibition." Cancer Research 69(17):6899-6905.

Tweddle, Deborah A., Archie J. Malcolm, Michael Cole, Andrew D. J. Pearson, and John Lunec. 2001. "P53 Cellular Localization and Function in Neuroblastoma: Evidence for Defective G1 Arrest despite WAF1 Induction in MYCN-Amplified Cells." American Journal of Pathology 158(6):2067-77.

Valkov, N. I. and D. M. Sullivan. 1997. "Drug Resistance to DNA Topoisomerase I and II Inhibitors in Human Leukemia, Lymphoma, and Multiple Myeloma." Seminars in Hematology 34(4 Suppl 5):4862.

Wander, Seth A., Dekuang Zhao, and Joyce M. Slingerland. 2011. "P27: A Barometer of Signaling Deregulation and Potential Predictor of Response to Targeted Therapies." Clinical Cancer Research 17(1):12-18.

Wang, Amy Y. and Hongtao Liu. 2019. "The Past, Present, and Future of CRM1/XPO1 Inhibitors." Stem Cell Investigation.

Wang, James C. 2002. "Cellular Roles of DNA Topoisomerases: A Molecular Perspective." Nature Reviews Molecular Cell Biology 3(6):430-40.

Wang, Wei, Anuradha Budhu, Marshonna Forgues, and Xin Wei Wang. 2005. "Temporal and Spatial Control of Nucleophosmin by the Ran-Crm1 Complex in Centrosome Duplication." Nature Cell Biology 7(8):823-30.

Wang, You, Yingying Wang, Jingying Xiang, Fang Ji, Yan Deng, Chunhui Tang, Shuyun Yang, Qinghua Xi, Rong Liu, and Wen Di. 2014. "Knockdown of CRM1 Inhibits the Nuclear Export of P27Kip1 Phosphorylated at Serine 10 and Plays a Role in the Pathogenesis of Epithelial Ovarian Cancer." Cancer Letters 343(1):6-13.

Watanabe, K., N. Takizawa, M. Katoh, K. Hoshida, N. Kobayashi, and K. Nagata. 2001. "Inhibition of Nuclear Export of Ribonucleoprotein Complexes of Influenza Virus by Leptomycin B." Pp. 31-42 in Virus Research. Vol. 77. Elsevier.

Watt, Pauline J. van der and Virna D. Leaner. 2010. "Regulation of Crm1 Expression in Cancer Cells." Pp. A33-A33 in. American Association for Cancer Research (AACR).

Van Der Watt, Pauline J., Christopher P. Maske, Denver T. Hendricks, M. Iqbal Parker, Lynette Denny, Dhirendra Govender, Michael J. Birrer, and Virna D. Leaner. 2009. "The Karyopherin Proteins, Crm1 and Karyopherin B1, Are Overexpressed in Cervical Cancer and Are Critical for Cancer Cell Survival and Proliferation." International Journal of Cancer 124(8):1829-40.

Wen, Wei, Judy L. Meinkotht, Roger Y. Tsien, and Susan S. Taylor. 1995. "Identification of a Signal for Rapid Export of Proteins from the Nucleus." Cell 82(3):463-73.

Yang, Qing, Michael P. Rout, and Christopher W. Akey. 1998. "Three-Dimensional Architecture of the Isolated Yeast Nuclear Pore Complex: Functional and Evolutionary Implications." Molecular Cell 1(2):223-34.

Yao, Yang, Yang Dong, Feng Lin, Hui Zhao, Zan Shen, Ping Chen, Yuan Jue Sun, Li Na Tang, and Shui Er Zheng. 2009. "The Expression of CRM1 Is Associated with Prognosis in Human Osteosarcoma." Oncology Reports 21(1):229-35. 
Ye, Ying and Baoan Li. 2006. "1'S-1'-Acetoxychavicol Acetate Isolated from Alpinia Galanga Inhibits Human Immunodeficiency Virus Type 1 Replication by Blocking Rev Transport." Journal of General Virology 87(7):2047-53.

Yue, Lu, Zhen Ni Sun, Ya Sai Yao, Zan Shen, Hai Bo Wang, Xiang Ping Liu, Fang Zhou, Jin Yu Xiang, Ru Yong Yao, and Hai Tao Niu. 2018. "CRM1, a Novel Independent Prognostic Factor Overexpressed in Invasive Breast Carcinoma of Poor Prognosis." Oncology Letters 15(5):7515-22.

Zamanian, M. and N. B. La Thangue. 1993. "Transcriptional Repression by the Rb-Related Protein P107." Molecular Biology of the Cell 4(4):389-96.

Zeng, Xin, He Huang, Keiko Tamai, Xinjun Zhang, Yuko Harada, Chika Yokota, Karla Almeida, Jianbo Wang, Brad Doble, Jim Woodgett, Anthony Wynshaw-Boris, Jen Chieh Hsieh, and Xi He. 2008. "Initiation of Wnt Signaling: Control of Wnt Coreceptor Lrp6 Phosphorylation/Activation via Frizzled, Dishevelled and Axin Functions." Development 135(2):367-75.

Zhang, Kejie, Michael Wang, Archito T. Tamayo, Sharon Shacham, Michael Kauffman, John Lee, Liang Zhang, Zhishuo Ou, Changping Li, Luhong Sun, Richard J. Ford, and Lan V. Pham. 2013. "Novel Selective Inhibitors of Nuclear Export CRM1 Antagonists for Therapy in Mantle Cell Lymphoma." Experimental Hematology 41(1):67-78.e4.

Zhang, Y. and Y. Xiong. 2001. "A P53 Amino-Terminal Nuclear Export Signal Inhibited by DNA DamageInduced Phosphorylation." Science 292(5523):1910-15.

Zheng, Yun, Sigal Gery, Haibo Sun, Sharon Shacham, Michael Kauffman, and H. Phillip Koeffler. 2014. "KPT-330 Inhibitor of XPO1-Mediated Nuclear Export Has Anti-Proliferative Activity in Hepatocellular Carcinoma." Cancer Chemotherapy and Pharmacology 74(3):487-95.

Zhou, Fang, Wensheng Qiu, Ruyong Yao, Jinyu Xiang, Xiaoxiao Sun, Shihai Liu, Jing Lv, and Lu Yue. 2013. "CRM1 Is a Novel Independent Prognostic Factor for the Poor Prognosis of Gastric Carcinomas." Medical Oncology 30(4):726.

Zhu, Jiangyu and Frank McKeon. 1999. "NF-AT Activation Requires Suppression of Crm1-Dependent Export by Calcineurin." Nature 398(6724):256-60. 


\section{Abbreviations}

\begin{tabular}{|c|c|}
\hline$\AA$ & Angstrom \\
\hline AAA & alanine-alanine-alanine \\
\hline APC & Adenomatous polyposis coli protein \\
\hline BME & 2-Mercaptoethanol \\
\hline BRCA1 & Breast cancer susceptibility protein 1 \\
\hline BARD1 & BRCA1-associated RING domain protein 1 \\
\hline C. thermophilum & Chaetomium thermophilum \\
\hline CBD & Cap binding domain \\
\hline CBP20 & Cap binding protein 20 \\
\hline CBP20 & Cap binding protein 80 \\
\hline CDK & Cyclin/cyclin-dependent kinase \\
\hline cFID & Consensus Induced Fit Docking \\
\hline cNLS & Classical NLS \\
\hline CRM1 & Chromosome Region Maintenance 1 \\
\hline$C t$ & Chaetomium thermophilum \\
\hline${ }^{C t}$ CRM1 & CRM1 from Chaetomium thermophilum \\
\hline C-terminal & Carboxy-terminal \\
\hline Da & Dalton \\
\hline DESY & Deutsches Elektronen-Synchrotron \\
\hline DNA & Deoxyribonucleic acid \\
\hline EM & Electron microscopy \\
\hline EMBL & European Molecular Biology Laboratory \\
\hline FG repeats & Phenylalanine-glycine repeats \\
\hline FG-nucleoporins & Phenylalanine-glycine nucleoporins \\
\hline FOXO & Forkhead box \\
\hline $\mathrm{FP}$ & Fluorescence polarization \\
\hline
\end{tabular}




\begin{tabular}{|c|c|}
\hline GDP & Guanosine-5'-diphosphate \\
\hline GSK-3 $\beta$ & Glycogen synthase kinase 3 beta \\
\hline GTP & Guanosine-5'-triphosphate \\
\hline H. sapiens & Homo sapiens \\
\hline $\mathrm{His}_{6}$ & Hexa histidine-tag \\
\hline HIV & Human immunodeficiency virus \\
\hline HIV-1 & Human immunodeficiency virus type 1 \\
\hline Hs & Homo sapiens \\
\hline${ }^{H s} \mathrm{CRM} 1$ & CRM1 from Homo sapiens \\
\hline${ }^{H s} \mathrm{CRM}^{\Delta}{ }^{\Delta}$ & HEAT 9 loop mutant and C-terminally truncated human CRM1 \\
\hline HTS & High throughput screening \\
\hline HUPB & Hold until publication \\
\hline $1 \mathrm{C}_{50}$ & Half-maximal inhibitory concentration \\
\hline IPTG & Isopropyl $\beta$-D1-thiogalactopyranoside \\
\hline $\mathrm{Kd}$ & Dissociation constants \\
\hline kDa & Kilodalton \\
\hline LC-MS & Liquid chromatography-mass spectrometry \\
\hline LMB & Leptomycin B \\
\hline M. musculus & Mus musculus \\
\hline$m^{7} G$ & 7-methylguanylate \\
\hline MBP & Maltose-binding protein \\
\hline MD & Molecular dynamics \\
\hline $\mathrm{MDa}$ & Megadalton \\
\hline MOE & Molecular operating environment \\
\hline mRNA & Messenger ribonucleic acid \\
\hline MWCO & Molecular weight cut off \\
\hline NEP & Nuclear export viral protein \\
\hline
\end{tabular}




\begin{tabular}{|c|c|}
\hline NES & Nuclear export signal \\
\hline nESI & Nano-electrospray IONIZATION \\
\hline NFAT & Nuclear factor of activated T-cells \\
\hline NLS & Nuclear Localization Signal \\
\hline NPC & Nuclear pore complex \\
\hline N-terminal & Amino-terminal \\
\hline NTF2 & Nuclear transport factor 2 \\
\hline NUPS & Nucleoporins \\
\hline OD & Optical density \\
\hline PAA & Polyacrylic acid \\
\hline PAGE & Polyacrylamide gel electrophoresis \\
\hline PDB & Protein data bank \\
\hline PDB-ID & PDB identification code \\
\hline PEG & Polyethylene glycol \\
\hline PHAX & Phosphorylated adapter of RNA export \\
\hline $\mathrm{p} K_{a}$ & Negative base-10 logarithm of the acid dissociation constant \\
\hline PKI & Protein kinase $\mathrm{A}$ inhibitor \\
\hline Ran & Small GTPase Ras-related antigen \\
\hline RanBP1 & Ran binding protein 1 \\
\hline RanBP2 & Ran binding protein 2 \\
\hline RanGAP1 & Ran GTPase activating protein \\
\hline RanGEF & Ran guanine nucleotide exchange factor \\
\hline $\mathrm{Rb}$ & Retinoblastoma \\
\hline RCC1 & Regulator of chromosome condensation 1 \\
\hline RNA & Ribonucleic acid \\
\hline RNP & Ribonucleoprotein \\
\hline RRE & Rev response element \\
\hline
\end{tabular}




$\begin{array}{ll}\text { R-SH } & \text { Thiol moiety } \\ \text { rSUs } & \text { Ribosomal subunits } \\ \text { RT } & \text { Retention time } \\ \text { S. cerevisiae } & \text { Saccharomyces cerevisiae } \\ \text { SAXS } & \text { Small-angle X-ray scattering } \\ \text { Sc } & \text { Saccharomyces cerevisiae } \\ \text { ScCRM1 } & \text { CRM1 from Saccharomyces cerevisiae } \\ \text { SDS } & \text { Sodium dodecyl sulfate } \\ \text { SINEs } & \text { Selective inhibitors of nuclear export } \\ \text { snRNAs } & \text { Small nuclear RNAs } \\ \text { SPN1 } & \text { Snurportin 1 } \\ \text { TCEP } & \text { Tris(2-carboxyethyl)phosphine } \\ \text { UsnRNP } & \text { Uridine-rich small nuclear RNP } \\ \text { VLV } & \text { valine-leucine-valine } \\ \text { VRNP } & \text { viral ribonucleoproteins } \\ \text { w/V } & \text { Wight to volume ratio } \\ \text { wt } & \text { Wild type }\end{array}$




\section{Acknowledgements}

This work could not have been accomplished without a great deal of guidance and support. I would like to express my sincere gratitude to those people who provided me with all means of support that I needed during my work on this project.

The work presented in this thesis was carried out in the Department for Molecular Structural Biology at the Göttingen Centre for Molecular Biosciences (GZMB). I am sincerely grateful for the opportunity to work on this project, given to me by my supervisor Prof. Dr. Ralf Ficner who provided a great and a productive research environment that made this thesis possible. I truly appreciate his sincere interest in this project and his outstanding and continuous support. Furthermore, I would like to thank him for the multiple opportunities to present my work to the scientific community at local and international conferences.

I am also grateful to Prof. Dr. Ralph Kehlenbach and Prof. Dr. Kai Tittmann for their participation in my Thesis Advisory Committee and providing useful input during the thesis committee meetings. Furthermore, I would like to thank Prof. Dr. Heike Krebber, Prof. Dr. Gerhard Braus, and Prof. Dr. Peter Rehling for being part of my extended examination board.

Much gratitude for Dr. Thomas Monecke for his constant guidance, support, motivation, and for all the invested time in guiding me especially at the beginning of my work on this project. His dynamism, vision, sincerity, and motivation have deeply inspired me. He encouraged me to not only grow as a researcher but also as an independent thinker. My deepest appreciation and special thanks go to Dr. Piotr Neumann for sparing his valuable time whenever I approached him. He has been there providing his heartfelt support and an outstanding advice especially in crystallographic matters. He has given me invaluable guidance, motivation, inspiration, and suggestions which had a great impact on the quality of the work presented in this thesis. I would also like to thank him for his empathy and great sense of humor which made working days, especially during synchrotron trips, truly enjoyable. I would also like to thank Dr. Achim Dickmanns who always answered my questions on the topic of nuclear transport and was always very helpful with his expertise on laboratory work. I am also very thankful to Dr. Oliver Valerius and Dr. Kerstin Schmitt from the Mass Spectrometry - Yeast group for their great assistance with protein mass spectrometry analysis.

I owe a very important debt to all members of the MSB department for their friendship and creating a welcoming working atmosphere. Especially I thank Jana, Khati, Marieke, Katharina, Flo, 
and Tim for their support, friendship, and all the great moments we had at synchrotron trips, lunchtime, conferences, and excursions. I also would like to thank my students Lennart Mohnike, Benjamin Gnoth, Lyova Mamoyan, and Marie Wensien for the opportunity of working together and participate in their scientific education. I also thank Marita Kalck and Susanne van Beckum for their patience answering thousands of bureaucratic questions I had. I would also like to thank Daniel Weinrich for helping me with any technical issue that I had in the lab or in the office and for his great effort in explaining X-ray - related safety instructions. I also extend my thanks to Annette Berndt, Beate Heinze, and Maja Mechmershausen for making work in the lab much easier with their great assistance and for the nice talks that really improved my knowledge in the German language.

Furthermore, I would like to thank Mohamed Hamed from the group of Prof. Dr. Ralph Kehlenbach and Roshan Perera from the group of Dr. Roland Dosch for the insightful scientific discussions and their long-lasting friendship.

In these lines, I would like to thank my friends, who always supported me, gave me good advice, and made my life joyful. Thank you for being there for me! Lastly and most importantly I would like to thank the biggest source of my strength, my family. The blessing of my parents, the support of my brothers, and the love and care of my sisters have all made a tremendous contribution in helping me reach this stage in my life. I dedicate this thesis to them. 
